\title{
Microbial Physiology of Subsurface Aquifers at SRS (U)
}

by

C. B. Fliermans,

Westinghouse Savannah River Company

Savannah River Site

Aiken, South Carolina 29808

J. House

M. M. Franck

DOE Contract No. DE-AC09-89SR18035

This paper was prepared in connection with work done under the above contract number with the U.S.

Department of Energy. By acceptance of this paper, the publisher and/or recipient acknowledges the U.S.

Government's right to retain a nonexclusive, royalty-free license in and to any copyright covering this paper, along with the right to reproduce and to authorize others to reproduce all or part of the copyrighted paper. 



\title{
MICROBIAL PHYSIOLOGY OF SUBSURFACE AQUIFERS AT SAVANNAH RIVER SITE
}

\author{
Carl B. Fliermans, John House and Marilyn M.Franck \\ Biotechnology Group \\ Westinghouse Savannah River Technology Center \\ Aiken, SC, USA
}

\section{Milestone: TCC07 Characterization of Microorganisms in SRS Aquifers}

\section{EXECUTIVE SUMMARY:}

Subsurface microbial communities differ in their physiological characteristics in relationship to depth and whether the organisms are associated with sediments or ground water. Investigations were undertaken to determine the response of the microbial communities present in the aquifers of SRS to selected organic compounds. Ground water samples were collected from P24, P-28 and P-29 well clusters. Selected microbiological, physical and chemical parameters were measured for each water sample. Triplicate ground water samples $(150 \mu \mathrm{l})$, without concentration, were injected into each of the 96 wells of the GN-Biolog® plates. Plates were incubated for 3 weeks at in situ ground water temperatures and the reactions to the organic compounds present in the GN-Biolog ${ }^{\circledR}$ plates were evaluated. The data indicate that microbial populations and/or specific extracellular enzyme systems are present in the ground waters and degrade the compounds present in the Biolog ${ }^{\circledR}$ plates. The data indicate that given the limited samples, one can observe differences among the aquifers with regard to physiological parameters. The microbial consortia associated with surface aquifers are clearly different in their physiology when compared to the deep Black Creek or Middendorf aquifers. The investigation indicates that Biolog ${ }^{\circledR}$ technology is a useful tool to measure the physiological response of the microbial community present in the ground waters of SRS. Further it allows one to evaluate the versatility of the microbial subsurface ecosystem and to assess the potential for each of the aquifers to provide either enhanced or intrinsic bioremediation. Additionally, aquifers may be characterized based on their microbial community and subsequent activity rather than solely on the geological structure of the subsurface.

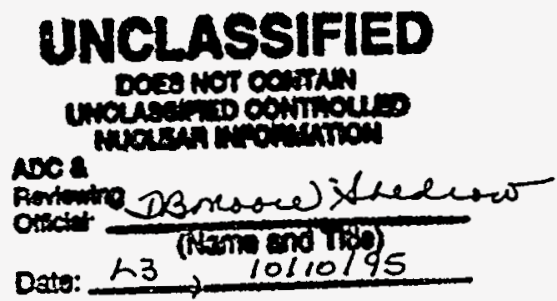




\section{INTRODUCTION}

As a result of various human activities, particularly those involving land disposal of chemical waste, the quality of ground water has become increasingly threatened. The total annual world production of synthetic organic chemicals is over 300 million tons, and more than 1000 new compounds are marketed each year (Fewson, 1988). It has been estimated that over 57 million metric tons of hazardous industrial waste were produced in the US during 1980 alone (Keswick, 1984). For these reasons, pollution of ground water due to hazardous waste is becoming a very serious problem with more than 10 million Americans using tap water with contaminants that exceed EPA standard levels (Knox, 1988).

Bacteria capable of degrading toxic organic chemicals have been isolated from aquatic systems, deep wells, and terrestrial subsurface sites (Little, et al., 1988; Fliermans and Balkwill, 1988; Fliermans et al., 1988; Efraymson and Alexander, 1991.) These microbial consortia represent resources that are physiologically diverse and economically important for genomic stability and the enhancement of bioremediation activities in the terrestrial subsurface. Procedures previously used to determine the microbial diversity of subsurface environments and their ability to degrade or mineralize toxic contaminants required repeated enrichments and exposure of organisms to various concentrations of toxic substances for weeks or months (Villarreal et al., 1991; Hutchins, 1991). Because the use of heterotrophic aerobic bacteria in the bioremediation of contaminated sites is increasing (Abramowicz and Brennan, 1990; Anonymous, 1989), the capacity to quickly characterize potential degraders, access remediation strategies and evaluate subsurface communities and their recovery during and after perturbations will enhance bioremediation technologies.

The Savannah River Site is a 310 square mile facility owned by the U.S. Department of Energy and operated by the Westinghouse Savannah River Company. The site is near Aiken, South Carolina, and has been operated as a nuclear production facility for DOE since 1950 . The production processes carried out over the past 40 years have generated considerable waste and waste sites. This waste includes radiological, heavy metals, organic solvents, sanitary landfills and other types of mixed wastes. Many contaminated environments at SRS have been identified including both surface water and soils, subsurface sediment and ground water. Cleanup of these wastes and waste sites has become a top priority for DOE. Due to the large number of waste sites and large volume of contaminants at many of these sites a considerable amount of time and money will be required to complete the mandated cleanup. Thus, another priority stemming from this cleanup program is to develop and demonstrate new and innovative technologies that may decrease costs, decrease time, decrease environmental impact and/or result in a cleaner end point.

This report describes a rapid screening procedure used to test the enzymatic capabilities of bacterial consortia in ground water from well clusters P-24, P-28 and P-29. The response of these ground water microbial communities were evaluated for their ability to degrade selected organic substrates in the degradative process. Screening procedures using Biolog ${ }^{\circledR}$ multiwell plates (Biolog ${ }^{\circledR}$, Hayward, CA) are versatile, flexible, and suitable for testing a wide range of mixtures and concentrations of in situ enzymatic patters, toxic chemicals and selected microorganisms. Biolog ${ }^{\circledR}$ technology was initially developed as a rapid, broad spectrum method for the biochemical identification of clinical microorganisms. Demand and creative application of this technology has resulted in the development of Biolog ${ }^{\circledR}$ plates for Gram-negative and Gram-positive bacteria, for yeast and Lactobacillus sp. The Biolog ${ }^{\circledR}$ identification library now has more than 1000 environmental species and strains (Biolog ${ }^{\circledR}, 1994$ Literature).

Microbial ecologists have extended the use of Biolog ${ }^{\circledR}$ plates from the identification of culture isolates to a tool for quantifying the metabolic patterns of mixed 
cultures, consortia and entire microbial communities. Patterns that develop on Biolog ${ }^{\circledR}$ microplates are a result of the oxidation of the substrates by microorganisms in the inoculum and the subsequent reduction of the tetrazolium dye to form a color whose intensity is in response to detectable reactions. Depending upon the functional enzymes present in the isolate or community one of a possible $4 \times 10^{28}$ patterns can be expressed (Biolog ${ }^{\circledR}, 1991$ ). The patterns are distinctive for isolates of different species and are now being used to distinguish the physiological ecology of various microbial communities.

Biolog ${ }^{\circledR}$ technology offers a unique capacity to measure functional aspects of bacterial enzyme activity in a reproducible and quantifiable way. Biolog ${ }^{\circledR}$ plate patterns develop due to enzyme activity in each positive well. The enzyme activity of pure cultures enables the identification of the isolates based on the phenological patterns. When water samples collected from natural aquatic systems are inoculated into Biolog ${ }^{\circledR}$ plates the resulting patterns are reflective of the enzymes expressed by the functioning microbial community and may be defined as ecologically functional or ecofunctional enzymes.

Ecofunctional enzymes (EFE) are enzymes which are being expressed or used by or within a microbial community in order to enable individuals or microbial populations to survive, maintain or grow. Alternatively, EFE may be latent enzymes ready for expression under more favorable or opportunistic conditions. Such enzymes are present in every microbial community, and it is expected that the suite of EFE expressed at any moment by the microbial communities present in subsurface groundwaters is a direct reflection of the dominant and contributing microbial populations of that community and of the environmental factors impacting the ecosystem at the time of sampling.

\section{MATERIAL and METHODS}

Ground water samples were collected aseptically on a monthly schedule from 8 wells in the P-24 cluster; 6 wells in the P-28 cluster and 7 wells in the P-29 cluster. The well location, aquifer details and well construction features are given in Figure 1 and Table 1 , respectively. Wells were sampled according to established protocol whereby either four (4) well volumes were pumped and/or chemical and physical parameters were stabilized before samples were collected. Four liter samples were collected. Each ground water sample was inoculated, without concentration, into triplicate GN-Biolog ${ }^{\circledR}$ (Biolog ${ }^{\circledR}$, Hayward, CA) microtiter plates at a level of $150 \mu \mathrm{l}$ per well. The plates were enclosed in sterile Whirl-Pak bags to prevent moisture loss and then incubated at $21^{\circ} \mathrm{C}$ (in situ mean ground water temperatures). After 21 days of incubation, plates were read on a microtiter plate reader $(590 \mathrm{~nm})$ used in conjunction with MicroLog 2, Release 2.01 software (Biolog ${ }^{\circledR}$, Hayward, CA). Data were collected as an optical density (OD) value for each of the 96 wells of the microtiter plate normalized against the control well, A1. If the A1 well (no nutrient source) had an OD value of 0.40 or over, the data for the plate was discarded. Data were analyzed using prepared programs for the Macintosh, including Systat version 5.1 (Systat, Inc., Evanston, IL) and JMP, version 2 (SAS Institute Inc., Cary, NC) and using statistical tests as described by Zar (1984). The Tukey-Kramer test was used to test multiple comparisons of means. Any statistical probability equal to or less than 0.05 was considered significant.

\section{RESULTS}

Before each of the cluster wells could be sampled, each of the wells had to be refurbished and refitted with submersible pumps, Such reworking of the wells required a considerable amount of time, greater than 9 months, so that the biological assessment of 
FORMATIONS

$$
\begin{gathered}
\text { Tobacco Road } \\
\text { Dry Branch } \\
\text { McBean } \\
\text {. Congaree } \\
\text { PeeDee } \\
\text { Black Creek } \\
\text { Middendorf }
\end{gathered}
$$

\section{NUMBER OF SAMPLES}
9
15
0
15
13
14
24

Biolog ${ }^{\circledR}$ redox technology is the basic component of the rapid screening procedure and is based on tetrazolium dye reduction as an indicator of enzyme systems capable of sole-carbon source utilization(Bochner, 1989 a, b; Garland Mills, 991). Although originally designed to identify clinical bacterial isolates using 95 different sole carbon sources, the technology has been extended by Biolog ${ }^{\circledR}$ to identify environmental isolates, MPN of soil and ground water habitats (Wear, 1993); and rapid screening of bacteria capable of degrading toxic organic compounds (Gorden et al., 1993).

The basic procedures described here uses Biolog GN plates. Biolog GN and GP plates contain the same minimal nutritional factors along with a different organic substrate (Table 2) in each of the 95 wells in a microtiter plate. MT plates differ in that they have no organic substrates in any wells but only minimal nutritional factors as well as the tetrazolium dye present in each well. The oxidized tetrazolium dye contained in each well turns purple when reduced as a response to bacterial metabolic activity.

Unconcentrated ground water samples from each of the P-24, P-28 and P-29 cluster wells were inoculated into each microtiter plate well. The plates were incubated at ground water temperature for three weeks, and the color changes indicative of the characteristic metabolic patterns were recorded. Although the 95 different carbon sources in Biolog GN plates were pre-selected specifically for characterizing and differentiating Gram-negative and Gram-positive aerobic bacteria, respectively, they are also useful in demonstrating metabolic patterns for mixed cultures. Carbon sources in the Biolog ${ }^{\circledR}$ plates are dominated by 28 carbohydrates, 24 carboxylic acids, and 20 amino acids plus various amides, aromatic chemicals, and other compounds (Table 2). The A-1 well of the microtiter plate has no substrate, and thus no nutrient source with which to initiate a metabolic reaction with the indicator dye, and thus serves as a negative control.

The Biolog ${ }^{\circledR}$ automated plate reader was factory programmed to sense a threshold optical density value for each plate based on the color of the A-1 reference control well. Thus, the optical density readings which determined each inoculum pattern, were significantly above the threshold level. Ground water samples from 3 cluster wells were sampled monthly for a period of 2 months. The data are represented as the optical density for each of the enzyme systems capable of degrading the organic compounds present in the Biolog ${ }^{\circledR}$ assays. Each of the 95 compounds were analyzed for each of the cluster wells. The data indicate that selected aquifers of the cluster wells were variable in their response. The microbial community present in the ground water changed their activity against specific groups of compounds as well as stereo isomers of selected amino acids. The data provides data and information into the understanding and modification of the microbial ecology of subsurface environments.

AQUIFER DEPTH. Each of the major aquifers are plotted with respect to depth in Figure 2. The data indicate that the depth of each of the aquifers differ and span a formation. Both the Black Creek and Middendorf aquifer formations are significantly different from the mean depth of the aquifers sampled. Because the well clusters are located in different regions of SRS the formation depth are not the same. Each of the major compound groups as shown in Table 2 were analyzed for the EFE systems expressed at each sampling period for each of the sampled wells. 
AQUIFER TEMPERATURE. Each of the major aquifers are plotted with respect to in situ ground water temperatures in Figure 3. The depths of the aquifers were not to the extent that elevated temperatures were observed. The deeper Black Creek and Middendorf aquifers were statistically elevated above the mean temperature $\left(21^{\circ} \mathrm{C}\right)$ of the aquifers.

AQUIFER OXYGEN. Each of the major aquifers are plotted with respect to in situ oxygen content in Figure 4. All of the aquifers are oxygenated with the deeper aquifers of the Black Creek and Middendorf having a mean oxygen content of 1.3 and $2.2 \mathrm{ppm}$, respectively. PeeDee was the only aquifer that has anaerobic conditions with a mean concentration of less than $0.02 \mathrm{ppm}$. The shallower aquifers of the Tobacco Road, Dry Branch, Congaree, and McBean average 7.9, 8.3,7.6 and $8.8 \mathrm{ppm}$, respectively.

AQUIFER CONDUCTIVITY. Each of the major aquifers are plotted with respect to in situ conductivity content in Figure 5. The data demonstrates that there is no significant difference in conductivity among the aquifers.

AQUIFER pH. Each of the major aquifers are plotted with respect to in situ $\mathrm{pH}$ values as shown in Figure 6. All of the aquifers except the McBean have acidic $\mathrm{pH}$ values. The average $\mathrm{pH}$ of the aquifers is 6.25. The McBean aquifer has shown elevated $\mathrm{pH}$ values averaging 8.63 and may reflect the intrusion and influence of grout into the aquifer system.

AQUIFER REDOX VALUES. Each of the major aquifers are plotted with respect to in situ redox values as shown in Figure 7. None of the aquifers were under a negative redox although PeeDee, Middendorf and Black Creek aquifers were statistically lower than the mean redox of $235 \mathrm{mv}$.

AQUIFER TOTAL BACTERIAL COUNTS. Each of the major aquifers are plotted with respect to in situ total bacterial counts per milliliter of ground water are shown in Figure 8. PeeDee aquifer was statistically elevated over the other aquifers, while each of the other aquifers were not statistically different form each other. Thus the inoculation of the Biolog ${ }^{\circledR}$ plates with ground water from each of the aquifers contained the same number of total bacteria within statistical significance.

CARBOHYDRATE UTILIZATION. The carbohydrate group was composed of 28 different carbohydrates that were utilized by the microbial consortia in the ground water from each of the cluster wells. Each of the major aquifers are plotted with respect to in situ carbohydrate utilization as shown in Figure 9. Carbohydrates were utilized most efficiently in the PeeDee and Tobacco Road aquifers. All but the Black Creek and the Middendorf aquifers were distinguished from each other on the basis of their carbohydrate utilization.

Figure 9a. N-Acetyl-D-galactosamine. The microbial community of the groundwater of the shallowest aquifer, Tobacco Road, demonstrated the greatest utilization activity for this specific carbohydrate. None of the other aquifers were significantly different from each other when measured at the 0.05 confidence level.

Figure 9b. N-Acetyl-D-glucosamine. The microbial community of the groundwater of the Dry Branch aquifer demonstrated the greatest utilization activity for this specific carbohydrate. None of the other aquifers were significantly different from each other when measured at the 0.05 confidence level.

Figure 9c. Adonitol. None of the aquifers were significantly different from each other when measured at the 0.05 confidence level.

Figure 9d. L-Arabinose. The microbial community of the ground water of the shallowest aquifer, Tobacco Road, demonstrated the greatest utilization activity for this specific carbohydrate. None of the other aquifers were significantly different from each other when measured at the 0.05 confidence level.

Figure 9e. D-Arabitol. The microbial community of the ground water of the two shallowest aquifers, Tobacco Road and Dry Branch demonstrated the greatest utilization activity for this specific carbohydrate. None of the other aquifers were significantly different from each other when measured at the 0.05 confidence level.

Figure 9f. Cellobiose. The microbial community of the ground water of the shallow aquifer, Dry Branch, demonstrated the greatest utilization activity for this specific 
carbohydrate, although the levels of utilization were not statistically significant at the 0.05 level. None of the other aquifers were significantly different from each other when measured at the 0.05 confidence level.

Figure 9g. i-Erthritol. None of the aquifers were significantly different from each other when measured at the 0.05 confidence level.

Figure 9h. D-Fructose.. The microbial community of the ground water of the shallow aquifer, Dry Branch, demonstrated the greatest utilization activity for this specific carbohydrate, although the levels of utilization were not statistically significant at the 0.05 level. None of the other aquifers were significantly different from each other when measured at the 0.05 confidence level.

Figure 9i. L-Fructose. None of the aquifers were significantly different from each other when measured at the 0.05 confidence level.

Figure 9j. D-Galactose. The microbial community of the ground water of the two shallowest aquifers, Tobacco Road and Dry Branch demonstrated the greatest utilization activity for this specific carbohydrate. None of the other aquifers were significantly different from each other when measured at the 0.05 confidence level.

Figure 9k. Gentiobiose. None of the aquifers were significantly different from each other when measured at the 0.05 confidence level.

Figure $91 \alpha$-D-Glucose. The microbial community of the ground water of the two shallowest aquifers, Tobacco Road and Dry Branch demonstrated the greatest utilization activity for this specific carbohydrate. None of the other aquifers were significantly different from each other when measured at the 0.05 confidence level.

Figure 9m. m-Inositol. None of the aquifers were significantly different from each other when measured at the 0.05 confidence level.

Figure 9n. $\alpha-D$-Lactose. None of the aquifers were significantly different from each other when measured at the 0.05 confidence level.

Figure 90. Lactulose. None of the aquifers were significantly different from each other when measured at the 0.05 confidence level.

Figure 9p. Maltose. None of the aquifers were significantly different from each other when measured at the 0.05 confidence level.

Figure 9q. D-Mannitol. None of the aquifers were significantly different from each other when measured at the 0.05 confidence level.

Figure 9r. D-Mannose. None of the aquifers were significantly different from each other when measured at the 0.05 confidence level.

Figure 9s. D-Melibiose. None of the aquifers were significantly different from each other when measured at the 0.05 confidence level.

Figure 9t. $\beta$-Methyl D- Glucoside. The microbial community of the ground water of the shallowest aquifer, Tobacco Road, demonstrated the greatest utilization activity for this specific carbohydrate. The microorganisms in the Tobacco Road aquifer microorganisms were significantly greater in their activity when compared to the PeeDee or Black Creek aquifers. None of the other aquifers were significantly different from each other when measured at the 0.05 confidence level.

Figure 9u. D-Psicose. None of the aquifers were significantly different from each other when measured at the 0.05 confidence level.

Figure 9v. D-Raffinose. None of the aquifers were significantly different from each other when measured at the 0.05 confidence level.

Figure 9w. L-Rhamnose. Only the Black Creek and Congaree aquifers differ significantly in their ability to utilize this carbohydrate. None of the other aquifers were significantly different from each other when measured at the 0.05 confidence level.

Figure 9x. D-Sorbitol. The microbial community of the ground water of the shallowest aquifer, Tobacco Road, demonstrated the greatest utilization activity for this specific carbohydrate. The microorganisms in the Tobacco Road aquifer microorganisms were significantly greater in their activity when compared to the PeeDee or Black Creek 
aquifers. None of the other aquifers were significantly different from each other when measured at the 0.05 confidence level.

Figure 9y. Sucrose. The microbial community of the ground water of the two shallowest aquifers, Tobacco Road and Dry Branch demonstrated the greatest utilization activity for this specific carbohydrate. None of the other aquifers were significantly different from each other when measured at the 0.05 confidence level.

Figure 9z. D-Trehalose. The microbial community in the PeeDee and Dry Branch aquifers were significantly different in their activity for this specific carbohydrate. None of the other aquifers were significantly different from each other when measured at the 0.05 confidence level.

Figure 9aa. Turanose. None of the aquifers were significantly different from each other when measured at the 0.05 confidence level.

Figure 9bb. Xylitol. The microbial community of the ground water of the shallowest aquifer, Tobacco Road, demonstrated the greatest utilization activity for this specific carbohydrate. None of the other aquifers were significantly different from each other when measured at the 0.05 confidence level.

\section{SUMMARY for CARBOHYDRATES}

None of the 28 different carbohydrates employed in these two sampling periods demonstrated any consistent patterns of activity. The data indicate that the microorganisms in the shallower aquifers, Tobacco Road and Dry Branch, are the most activity in the utilization of these carbohydrates.

ESTER UTILIZATION. The ester group was composed of 2 different compounds. These compounds were utilized by the microbial consortia in the ground water from each of the major aquifers and are plotted as a composite with respect to in situ ester utilization as shown in Figure 10. Esters were utilized most efficiently in the Tobacco Road and Dry Branch aquifers. None of the other aquifers were distinguished from each other on the basis of their ester utilization.

Figure 10a. Mono-Methylsuccinate. The microbial community of the ground water of the two shallowest aquifers, Tobacco Road and Dry Branch demonstrated the greatest utilization activity for this specific ester. These two aquifers were significantly different from the Black Creek and the PeeDee aquifers. None of the other aquifers were significantly different from each other when measured at the 0.05 confidence level.

Figure 10b. Methyl Pyruvate. The microbial community of the ground water of the two shallowest aquifers, Tobacco Road and Dry Branch demonstrated the greatest utilization activity for this specific ester. These two aquifers were significantly different from the Black Creek and the PeeDee aquifers. None of the other aquifers were significantly different from each other when measured at the 0.05 confidence level.

\section{SUMMARY for ESTERS}

None of the esters employed in these two sampling periods demonstrated any consistent patterns of activity. The data indicate that the microorganisms in the shallower aquifers, Tobacco Road and Dry Branch, are the most activity in the utilization of these esters.

POLYMER UTILIZATION. The polymer group was composed of 5 different compounds. These compounds were utilized by the microbial consortia in the ground water from each of the major aquifers and are plotted as a composite with respect to in situ polymer utilization as shown in Figure 11. Polymers were utilized most efficiently in the Tobacco Road and Dry Branch aquifers. None of the other aquifers were distinguished from each other on the basis of their polymer utilization.

Figure 11a. Glycogen. None of the aquifers were significantly different from each other when measured at the 0.05 confidence level. 
Figure 11b. $\alpha$-Cyclodextrin. None of the aquifers were significantly different from each other when measured at the 0.05 confidence level.

Figure 11c. Dextrin. The microbial community of the shallow aquifer, Dry Branch, demonstrated the greatest utilization activity for this specific polymer. The microbial community in the PeeDee aquifer was significantly different from the Congaree, and Dry Branch aquifers. The remaining aquifers were not significantly different from each other when measured at the 0.05 confidence level.

Figure 11d. Tween 80. The microbial community of the ground water of the two shallowest aquifers, Tobacco Road and Dry Branch demonstrated the greatest utilization activity for this specific polymer. These two aquifers were significantly different from the Congaree, Middendorf and the PeeDee aquifers. The microbial community from the Black Creek, Congaree, Middendorf and PeeDee aquifers were not significantly different from each other when measured at the 0.05 confidence level.

Figure 11e. Tween 40. The microbial community of the ground water of the two shallowest aquifers, Tobacco Road and Dry Branch demonstrated the greatest utilization activity for this specific polymer. The microbial community from the Black Creek, Congaree, Middendorf and PeeDee aquifers were not significantly different from each other when measured at the 0.05 confidence level.

\section{SUMMARY for POLYMERS}

None of the 5 different polymers employed in these two sampling periods demonstrated any consistent patterns of activity. The data indicate that the microorganisms in the shallower aquifers, Tobacco Road and Dry Branch, are the most activity in the utilization of these polymers.

CARBOXYLIC ACID UTLIZATION. The carboxylic acid group was composed of 24 different compounds. These compounds were utilized by the microbial consortia in the ground water from each of the major aquifers and are plotted as a composite with respect to in situ carboxylic acid utilization as shown in Figure 12. Carboxylic acids were utilized most efficiently in the two shallowest aquifers, Tobacco Road and Dry Branch. The microbial community of the PeeDee aquifer was significantly lower than any of the other tested aquifers. There was no difference observed in the microbial activity between the two deepest aquifers, Middendorf and Black Creek. The data indicate that the microbial community of the various aquifers differ with increasing depth.

Figure 12a. Acetic Acid. None of the aquifers were significantly different from each other when measured at the 0.05 confidence level.

Figure 12b. cis-Aconitic Acid. The microbial community of the Dry Branch aquifer demonstrated the greatest utilization activity for this specific carboxylic acid. The remaining aquifers were not significantly different from each other when measured at the 0.05 confidence level.

Figure 12c. Citric Acid. The microbial community of the ground water of the two shallowest aquifers, Tobacco Road and Dry Branch demonstrated the greatest utilization activity for this specific carboxylic acid. The remaining aquifers were not significantly different from each other when measured at the 0.05 confidence level.

Figure 12d. Formic Acid. None of the aquifers were significantly different from each other when measured at the 0.05 confidence level.

Figure 12e. D-Galactonic Acid Lactone. The microbial community of the ground water of the shallowest aquifer, Tobacco Road, demonstrated the greatest utilization activity for this specific carboxylic acid. None of the other aquifers were significantly different from each other when measured at the 0.05 confidence level.

Figure 12f. D-Galacturonic Acid. The microbial community of the ground water of the shallowest aquifer, Tobacco Road, demonstrated the greatest utilization activity for 
this specific carboxylic acid. None of the other aquifers were significantly different from each other when measured at the 0.05 confidence level.

Figure 12g. D-Gluconic Acid. The microbial community of the ground water of the two shallowest aquifers, Tobacco Road and Dry Branch demonstrated the greatest utilization activity for this specific carboxylic acid. The remaining aquifers were not significantly different from each other when measured at the 0.05 confidence level.

Figure 12h. D-Glucosaminic Acid. The microbial community of the ground water of the two shallowest aquifers, Tobacco Road and Dry Branch demonstrated the greatest utilization activity for this specific carboxylic acid. The remaining aquifers were not significantly different from each other when measured at the 0.05 confidence level.

Figure 12i. D-Glucuronic Acid. The microbial community of the ground water of the two shallowest aquifers, Tobacco Road and Dry Branch demonstrated the greatest utilization activity for this specific carboxylic acid. The remaining aquifers were not significantly different from each other when measured at the 0.05 confidence level.

Figure 12j. $\alpha$-Hydroxy Butyric Acid. The microbial community of the ground water of the shallowest aquifer, Tobacco Road, demonstrated the greatest utilization activity for this specific carboxylic acid. None of the other aquifers were significantly different from each other when measured at the 0.05 confidence level.

Figure 12k. $\beta$-Hydroxy Butyric Acid. The microbial community of the ground water of the two shallowest aquifers, Tobacco Road and Dry Branch demonstrated the greatest utilization activity for this specific carboxylic acid. The remaining aquifers were not significantly different from each other when measured at the 0.05 confidence level.

Figure 12I $\gamma$-Hydroxy Butyric Acid. The microbial community of the ground water of the shallowest aquifer, Tobacco Road, demonstrated the greatest utilization activity for this specific carboxylic acid. None of the other aquifers were significantly different from each other when measured at the 0.05 confidence level.

Figure 12m. $\rho$-Hydroxyphenylacetic Acid. None of the aquifers were significantly different from each other with respect to this carboxylic acid when measured at the 0.05 confidence level.

Figure 12n. Itaconic Acid. The microbial community of the ground water of the two shallowest aquifers, Tobacco Road and Dry Branch demonstrated the greatest utilization activity for this specific carboxylic acid. The remaining aquifers were not significantly different from each other when measured at the 0.05 confidence level.

Figure 120. $\alpha$-Ketobutyric Acid. The microbial community of the ground water of the shallow aquifer, Dry Branch, demonstrated the greatest utilization activity for this specific carboxylic acid. The remaining aquifers were not significantly different from each other when measured at the 0.05 confidence level.

Figure 12p. $\alpha$-Ketoglutaric Acid. The microbial community of the ground water of the two shallowest aquifers, Tobacco Road and Dry Branch demonstrated the greatest utilization activity for this specific carboxylic acid. The remaining aquifers were not significantly different from each other when measured at the 0.05 confidence level.

Figure 12q. $\alpha$-Ketovaleric Acid. The microbial community of the ground water of the shallow aquifer, Dry Branch, demonstrated the greatest utilization activity for this specific carboxylic acid. The Congaree and PeeDee aquifers differed statistically. The remaining aquifers were not significantly different from each other when measured at the 0.05 confidence level.

Figure 12r. D,L-Lactic Acid. The microbial community of the ground water of the two shallowest aquifers, Tobacco Road and Dry Branch demonstrated the greatest utilization activity for this specific carboxylic acid. The remaining aquifers were not significantly different from each other when measured at the 0.05 confidence level.

Figure 12s. Malonic Acid. The microbial community of the ground water of the Congaree demonstrated the greatest utilization activity for this specific carboxylic acid. The 
remaining aquifers were not significantly different from each other when measured at the 0.05 confidence level.

Figure 12t. Propionic Acid. The microbial community of the ground water of the shallowest aquifer, Tobacco Road, demonstrated the greatest utilization activity for this specific carboxylic acid. None of the other aquifers were significantly different from each other when measured at the 0.05 confidence level.

Figure 12u. Quinic Acid. The microbial community of the ground water of the two shallowest aquifers, Tobacco Road and Dry Branch demonstrated the greatest utilization activity for this specific carboxylic acid. The remaining aquifers were not significantly different from each other when measured at the 0.05 confidence level.

Figure 12v. D-Saccharic Acid. The microbial community of the ground water of the two shallowest aquifers, Tobacco Road and Dry Branch demonstrated the greatest utilization activity for this specific carboxylic acid. The remaining aquifers were not significantly different from each other when measured at the 0.05 confidence level.

Figure 12w. Sebacic Acid. The microbial community of the ground water of the two shallowest aquifers, Tobacco Road and Dry Branch demonstrated the greatest utilization activity for this specific carboxylic acid. The remaining aquifers were not significantly different from each other when measured at the 0.05 confidence level.

Figure 12x. Succinic Acid. The microbial community of the ground water of the two shallowest aquifers, Tobacco Road and Dry Branch demonstrated the greatest utilization activity for this specific carboxylic acid. The remaining aquifers were not significantly different from each other when measured at the 0.05 confidence level.

\section{SUMMARY for CARBOXYLIC ACID}

The 24 different carboxylic acids employed in these two sampling periods demonstrated a consistent pattern of activity. The data indicate that the microorganisms in the shallower aquifers, Tobacco Road and Dry Branch, are the most activity in the utilization of these carboxylic acids. The deeper aquifers are significantly different from the shallower aquifers in the ability of the in situ microbial community to use the selected carboxylic acids.

ALCOHOLS UTILIZATION. The group of alcohols was composed of 2 different compounds. These compounds were utilized by the microbial consortia in the ground water from each of the major aquifers and are plotted as a composite with respect to in situ alcohol utilization as shown in Figure 13. Alcohols were utilized most efficiently in the two shallowest aquifers, Tobacco Road and Dry Branch. The microbial community of the PeeDee aquifer was significantly lower than any of the other tested aquifers. There was no difference observed in the microbial activity between the two deepest aquifers,

Middendorf and Black Creek. The data indicate that the microbial community of the various aquifers differ with increasing depth.

Figure 13a. 2,3-Butanediol. The microbial community of the ground water of the deeper aquifers, Middendorf and Black Creek, demonstrated the greatest utilization activity for this specific alcohol. The remaining aquifers were not significantly different from each other when measured at the 0.05 confidence level.

Figure 13b. Glycerol. The microbial community of the ground water of the two shallowest aquifers, Tobacco Road and Dry Branch demonstrated the greatest utilization activity for this specific alcohol. The remaining deeper aquifers of Middendorf, Black Creek and PeeDee were significantly lower in their ability to utilize this specific alcohol.

AMIDES UTILIZATION. The group of amides was composed of 3 different compounds. These compounds were utilized by the microbial consortia in the ground water from each of the major aquifers and are plotted as a composite with respect to in situ amide utilization as shown in Figure 14. Amides were utilized most efficiently in the two shallowest aquifers, Tobacco Road and Dry Branch. The microbial community of the 
PeeDee aquifer was significantly lower than any of the other tested aquifers. There was no difference observed in the microbial activity between the two deepest aquifers, Middendorf and Black Creek. The data indicate that the microbial community of the various aquifers differ with increasing depth with respect to utilization of amides.

Figure 14a. Succinamic Acid. The microbial community of the ground water of the two shallowest aquifers, Tobacco Road and Dry Branch demonstrated the greatest utilization activity for this specific amide. This compound is utilized at the highest level for any of the compounds tested. The remaining aquifers were not significantly different from each other when measured at the 0.05 confidence level.

Figure 14b. Glucuronamide. None of the aquifers were significantly different from each other with respect to this carboxylic acid when measured at the 0.05 confidence level.

Figure 14c. Alaninamide. The microbial community of the ground water of the two shallowest aquifers, Tobacco Road and Dry Branch demonstrated the greatest utilization activity for this specific amide. The remaining aquifers were not significantly different from each other when measured at the 0.05 confidence level.

PHOSPHORYLATED COMPOUND UTILIZATION. The group of phosphorylated compound was composed of 3 different compounds. These compounds were utilized by the microbial consortia in the ground water from each of the major aquifers and are plotted as a composite with respect to in situ amide utilization as shown in Figure 15. Phosphorylated compound were utilized most efficiently in the two shallowest aquifers, Tobacco Road and Dry Branch. The microbial community of the PeeDee aquifer was significantly lower than any of the other tested aquifers except the Black Creek aquifer. There was no difference observed in the microbial activity between the two deepest aquifers, Middendorf and Black Creek. The data indicate that the microbial community of the various aquifers differ with increasing depth with respect to utilization of phosphorylated compounds tested.

Figure 15a. D,L- $\alpha$-Glycerol Phosphate. The microbial community of the ground water of the two shallowest aquifers, Tobacco Road and Dry Branch demonstrated the greatest utilization activity for this specific phosphorylated compound. The remaining aquifers were not significantly different from each other when measured at the 0.05 confidence level.

Figure 15b. Glucose-1-Phosphate. The microbial community of the ground water of the shallowest aquifer, Tobacco Road, demonstrated the greatest utilization activity for this specific phosphorylated compound. The remaining aquifers were not significantly different from each other when measured at the 0.05 confidence level.

Figure 15c. Glucose-6-Phosphate. None of the aquifers were significantly different from each other with respect to this carboxylic acid when measured at the 0.05 confidence level.

AMINO ACID UTLIZATION. The group of amino acid compounds was composed of 20 different compounds. These compounds were utilized by the microbial consortia in the ground water from each of the major aquifers and are plotted as a composite with respect to in situ amide utilization as shown in Figure 16. Amino acid compounds were utilized most efficiently in the two shallowest aquifers, Tobacco Road and Dry Branch. The microbial community of the PeeDee aquifer was significantly lower than any of the other tested aquifers. There was no difference observed in the microbial activity between the two deepest aquifers, Middendorf and Black Creek. The data indicate that the microbial community of the various aquifers differ with increasing depth with respect to utilization of amino acids tested.

Figure 16a. D-Alanine. None of the aquifers were significantly different from each other with respect to this amino acid when measured at the 0.05 confidence level. 
Figure 16b. L-Alanine. The microbial community of the ground water of the shallow aquifer, Dry Branch, demonstrated the greatest utilization activity for this specific amino acid. The remaining aquifers were not significantly different from each other when measured at the 0.05 confidence level.

Figure 16c. L-Alanyl Glycine. The microbial community of the ground water of the two shallowest aquifers, Tobacco Road and Dry Branch demonstrated the greatest utilization activity for this specific amino acid. The remaining aquifers were not significantly different from each other when measured at the 0.05 confidence level.

Figure 16d. L-Asparagine. The microbial community of the ground water of the two shallowest aquifers, Tobacco Road and Dry Branch demonstrated the greatest utilization activity for this specific amino acid. The remaining aquifers were not significantly different from each other when measured at the 0.05 confidence level.

Figure 16e. L-Aspartic Acid. The microbial community of the ground water of the three shallowest aquifers, Tobacco Road, Dry Branch and Congaree demonstrated the greatest utilization activity for this specific amino acid. The remaining aquifers were not significantly different from each other when measured at the 0.05 confidence level.

Figure 16f. L-Glutamic Acid. The microbial community of the ground water of the two shallowest aquifers, Tobacco Road and Dry Branch demonstrated the greatest utilization activity for this specific amino acid. The remaining aquifers were not significantly different from each other when measured at the 0.05 confidence level.

Figure 16g. Glycyl-L-Aspartic Acid. The microbial community of the ground water of the two shallowest aquifers, Tobacco Road and Dry Branch demonstrated the greatest utilization activity for this specific amino acid. The remaining aquifers were not significantly different from each other when measured at the 0.05 confidence level.

Figure 16h. Glycyl-L-Glutamic Acid. The microbial community of the ground water of the two shallowest aquifers, Tobacco Road and Dry Branch demonstrated the greatest utilization activity for this specific amino acid. The remaining aquifers were not significantly different from each other when measured at the 0.05 confidence level.

Figure 16i. L-Histidine. The microbial community of the ground water of the two shallowest aquifers, Tobacco Road and Dry Branch demonstrated the greatest utilization activity for this specific amino acid. The utilization of this amino acid by the microbial community in the PeeDee aquifer was significantly lower than the three shallowest aquifers, Tobacco Road, Dry Branch and Congaree. The remaining aquifers were not significantly different from each other when measured at the 0.05 confidence level.

Figure 16j. Hydroxy L-Proline. The microbial community of the ground water of the two shallowest aquifers, Tobacco Road and Dry Branch demonstrated the greatest utilization activity for this specific amino acid. The utilization of this amino acid by the microbial community in the PeeDee aquifer was significantly lower than the three shallowest aquifers, Tobacco Road, Dry Branch and Congaree. The remaining aquifers were not significantly different from each other when measured at the 0.05 confidence level.

Figure 16k. L-Leucine. The microbial community of the ground water of the two shallower aquifers, Dry Branch and Congaree demonstrated the greatest utilization activity for this specific amino acid. The remaining aquifers were not significantly different from each other when measured at the 0.05 confidence level.

Figure 16. L-Ornithine. The microbial community of the ground water of the two shallower aquifers, Dry Branch and Congaree demonstrated the greatest utilization activity for this specific amino acid. The remaining aquifers were not significantly different from each other when measured at the 0.05 confidence level.

Figure $16 \mathrm{~m}$. L-Phenylalanine. The microbial community of the ground water of the two shallowest aquifers, Tobacco Road and Dry Branch demonstrated the greatest utilization activity for this specific amino acid. The remaining aquifers were not significantly different from each other when measured at the 0.05 confidence level.

Figure 16n. L-Proline. The microbial community of the ground water of the two shallowest aquifers, Tobacco Road and Dry Branch demonstrated the greatest utilization 
activity for this specific amino acid. The remaining aquifers were not significantly different from each other when measured at the 0.05 confidence level.

Figure 160. L-Pyroglutamic. The microbial community of the ground water of the two shallowest aquifers, Tobacco Road and Dry Branch demonstrated the greatest utilization activity for this specific amino acid. The utilization of this amino acid by the microbial community in the PeeDee aquifer was significantly lower than the three shallowest aquifers, Tobacco Road, Dry Branch and Congaree. The remaining aquifers were not significantly different from each other when measured at the 0.05 confidence level.

Figure 16p. D-Serine. The microbial community of the ground water of the deepest aquifer, Middendorf, demonstrated the greatest utilization activity for this specific amino acid. The remaining aquifers were not significantly different from each other when measured at the 0.05 confidence level.

Figure 16q. L-Serine. The microbial community of the ground water of the two shallowest aquifers, Tobacco Road and Dry Branch demonstrated the greatest utilization activity for this specific amino acid. The utilization of this amino acid by the microbial community in the PeeDee aquifer was significantly lower than the three shallowest aquifers, Tobacco Road, Dry Branch and Congaree. The remaining aquifers were not significantly different from each other when measured at the 0.05 confidence level. A clear difference in utilization between the D-and L- form of Serine is apparent from the data.

Figure 16r. L-Theonine. The microbial community of the ground water of the two shallowest aquifers, Tobacco Road and Dry Branch demonstrated the greatest utilization activity for this specific amino acid. The remaining aquifers were not significantly different from each other when measured at the 0.05 confidence level.

Figure 16s. D, L-Carnitine. The microbial community of the ground water of the two shallowest aquifers, Tobacco Road and Dry Branch demonstrated the greatest utilization activity for this specific amino acid. The remaining aquifers were not significantly different from each other when measured at the 0.05 confidence level.

Figure 16t. $\gamma$-Amino Butyric Acid. . The microbial community in the ground water of the shallow aquifer, Dry Branch, demonstrated the greatest utilization activity for this specific amino acid. The utilization of this amino acid by the microbial community in the PeeDee aquifer was significantly lower than the shallow aquifers of Dry Branch and Congaree. The remaining aquifers were not significantly different from each other when measured at the 0.05 confidence level.

AROMATIC CHEMICAL UTILIZATION. The group of aromatic chemical compounds was composed of 4 different compounds. These compounds were utilized by the microbial consortia in the ground water from each of the major aquifers and are plotted as a composite with respect to in situ aromatic utilization as shown in Figure 17. Aromatic compounds were utilized most efficiently in the two shallowest aquifers, Tobacco Road and Dry Branch. The microbial community of the PeeDee aquifer was significantly lower than any of the other tested aquifers. There was no difference observed in the microbial activity between the two deepest aquifers, Middendorf and Black Creek. The data indicate that the microbial community of the various aquifers differ with increasing depth with respect to utilization of aromatic compounds tested.

Figure 17a. Inosine. The microbial community of the ground water of the shallowest aquifer, Tobacco Road, demonstrated the greatest utilization activity for this specific aromatic compound. The utilization of this aromatic by the microbial community in the PeeDee aquifer was significantly lower than the shallow aquifer of the Congaree. The remaining aquifers were not significantly different from each other when measured at the 0.05 confidence level.

Figure 17b. Urocanic Acid. The microbial community of the ground water of the two shallowest aquifers, Tobacco Road and Dry Branch demonstrated the greatest utilization activity for this specific aromatic compound. The utilization of this aromatic by 
the microbial community in the PeeDee aquifer was significantly lower than the shallow aquifer of the Congaree or the deeper Black Creek aquifer. The remaining aquifers were not significantly different from each other when measured at the 0.05 confidence level.

Figure 17c. Thymidine. None of the aquifers were significantly different from each other with respect to this aromatic compound when measured at the 0.05 confidence level.

Figure 17d. Uridine. None of the aquifers were significantly different from each other with respect to this aromatic compound when measured at the 0.05 confidence level.

BROMINATED CHEMICAL UTILIZATION. The group of brominated chemical compounds was composed of a single compound, Bromosuccinic Acid. This compound was utilized by the microbial consortia in the ground water from each of the major aquifers and are plotted as a composite with respect to in situ aromatic utilization as shown in Figure 18. Brominated compound was utilized most efficiently in the two shallowest aquifers, Tobacco Road and Dry Branch. There was no difference observed in the microbial activity between the two deepest aquifers, Middendorf and Black Creek.

AMINES UTILIZATION. The group of amine compounds was composed of 3 compounds. These compounds were utilized by the microbial consortia in the ground water from each of the major aquifers and are plotted as a composite with respect to in situ aromatic utilization as shown in Figure 19. Amine compounds were utilized most efficiently in the three shallowest aquifers, Tobacco, Road Dry Branch and Congaree. There was no difference observed in the microbial activity between the two deepest aquifers, Middendorf and Black Creek.

Figure 19a. Phenylethylamine. The microbial community of the ground water of the shallowest aquifer, Tobacco Road demonstrated the greatest utilization activity for this specific amine. None of the other aquifers were significantly different from each other with respect to this amine compound when measured at the 0.05 confidence level.

Figure 19b. 2-Amino Ethanol. The microbial community of the ground water of the three shallowest aquifers, Tobacco Road, Dry Branch and Congaree demonstrated the greatest utilization activity for this specific amino acid. The remaining aquifers were not significantly different from each other when measured at the 0.05 confidence level.

Figure 19c. Putrescine. None of the aquifers were significantly different from each other with respect to this amine compound when measured at the 0.05 confidence level.

\section{DISCUSSION}

The Biolog® data are very important in assessing the microbial activity of the subsurface community under in situ conditions. These data function in a variety of ways. It demonstrates that subsurface microbial populations are able to transform a number of different organic compounds with selected efficiencies. Such data can be utilized to establish baseline for microbial aquifer physiology. Thus if perturbations occur in the aquifer, such perturbations can be documented and the extent of recovery can be shown.

It is difficult to draw any overall conclusions from the sampling of each of these cluster wells only twice. There does appear that a trend exists whereby the shallower aquifers of Tobacco Roar, Dry Branch and Congaree respond differently to almost all of the compounds tested when compared to the deeper aquifers of the Black Creek and Middendorf. The PeeDee aquifer appears to be a transition are for many of the compounds tested. Only one compound, D-Serine was more effectively utilized in the deeper aquifer than in the shallow ones.

The data from this sampling indicate that the bacterial activity, physiological ecology, and ecofunctional enzyme patterns can be assessed and quantified. The data indicate that 
Biolog@ data are useful in evaluating both the chemicals that can be used to stimulate the subsurface microbial consortia and to determine when the consortia has been returned to preperturbation conditions.

\section{REFERENCES}

Abramowicz, D.A. and M.J. Brennan. 1990. Biological remediation of contaminated sediments. Abstract, Workshop of Biol. Remediation of Contaminated Sediments, Manitowoc, WI.

Anonymous. 1989. Environmental Protection Agency Workshop on Bioremediation. February, Athens, GA.

Biolog Inc. 1991. Microstation System, Biolog Inc., 3447 Investment Blvd., Hayward, CA.

Bochner, B. 1989a. Breathprints at the microbial level. ASM News 55:536-539.

Bochner, B. 1989b. Sleuthing out bacterial identities. Nature 339:157.

Efraymson, R.A. and M. Alexander. 1991. Biodegradation by an Arthrobacter species of hydrocarbons partioned into an organic solvent. Appl. Environ. Microbiol. 57:1441-1447.

Fewson, C. A. 1988. Biodegradation of xenobiotic and other persistent compounds: the causes of recalcitrance. Tibtech. 6: 148-153.

Fliermans, C. B. and D. L. Balkwill. 1989. Life in the terrestrial deep subsurface. BioScience 39: 370-377.

Garland, J.L. and A.L. Mills. 1991. Classification and characterization of heterotrophic microbial communities on the basis of patterns of community-level sole-carbonsource utilization. Appl. Environ. Microbiol. 57:2351-2359.

Gorden, R.W., T. C. Hazen and C. B. Fliermans. 1993. Use of Biolog® Technology for Hazardous Chemical Screening. Microbiological Techniques. 18: 329-347

Hutchins, S.R. 1991. Biodegradation of monoaromatic hydrocarbons by aquifer microorganisms using oxygen, nitrate, or nitrous oxide as the terminal electron acceptor. Appl. Environ. Microbiol. 57:2135-2140.

Keswick, B. H. 1984. Sources of ground water pollution. p. 39-64. In Bitton, G. and Gerba, C. P. (eds) Ground water Pollution Microbiology. John Wiley and Sons. New York, NY.

Knox, C. E. 1988. What's going on down there? Pervasive ground water contamination prompts new cleanup approaches. 134: 362-365.

Little, C.D., A.V. Palumbo, S.E. Herbes, M.E. Lidstrom, R.L. Tyndall, and P.J. Gilmer. 1988. Trichlorethylene biodegradation by a methane-oxidizing bacterium. Appl. Environ. Microbiol. 54:951-956.

Villarreal, D.T., F.F. Turco, and A. Konopka. 1991. Propachlor degradation by a soil bacterial community. Appl. Environ. Microbiol. 57:2135-2140.

Wear, J. E.., Jr. 1993. Environmental Diagnosytic Analysis of Ground Water Bacteria and Their Involvement in Utilization of Aromatic Compounds. Ph. D. Thesis. Wake Forest University. pgs. 186. 
TABLE 1. Physical and Chemical Data of the Sampled P Wells

\begin{tabular}{lccccrrrr} 
WEll ID & $\begin{array}{c}\text { SRS } \\
\text { North }\end{array}$ & $\begin{array}{c}\text { SRS } \\
\text { East }\end{array}$ & $\begin{array}{c}\text { EffWell } \\
\text { Depth } \\
(\mathrm{ft})\end{array}$ & $\begin{array}{c}\text { Temp } \\
\left({ }^{\circ} \mathrm{C}\right)\end{array}$ & $\begin{array}{c}\text { D.O. } \\
(\mathrm{ppm})\end{array}$ & $\begin{array}{c}\text { Cond. } \\
(\mu \mathrm{mh} / \mathrm{sec})\end{array}$ & pH & \multicolumn{1}{c}{$\begin{array}{c}\text { Redox } \\
(\mathrm{mv})\end{array}$} \\
P 24 A & 43142.2 & 66569.7 & 316.9 & 21.30 & 5.94 & 111 & 9.33 & 248.00 \\
P-24 B & 43127.8 & 66573.0 & 231.4 & 20.49 & 8.76 & 163 & 8.63 & 270.00 \\
P-24 C & 43112.8 & 66576.3 & 152.0 & 20.70 & 10.12 & 37 & 5.94 & 399.00 \\
P-24D & 43098.5 & 66579.5 & 66.8 & 20.38 & 8.44 & 28 & 5.35 & 400.00 \\
P-24TA & 43096.2 & 66565.2 & 974.1 & 21.92 & 0.08 & 71 & 5.83 & 35.00 \\
P-24TB & 43110.4 & 66561.7 & 806.9 & 21.63 & 0.90 & 75 & 6.06 & 131.00 \\
P-24TC & 43125.3 & 66558.2 & 597.0 & 21.37 & 0.01 & 78 & 5.91 & 88.00 \\
P-24TD & 43139.3 & 66554.9 & 501.9 & 21.07 & 1.20 & 96 & 6.11 & 133.00 \\
& & & & & & & & \\
P-28 A & 79275.2 & 55487.9 & 343.1 & 20.24 & 0.01 & 69 & 5.56 & 154 \\
P-28TA & 79284.3 & 55441.1 & 781.9 & 21.77 & 3.78 & 37 & 5.12 & 337 \\
P-28TB & 79288.9 & 55471.4 & 642.2 & 21.44 & 0.91 & 49 & 5.23 & 161 \\
P-28TC & 79291.1 & 55485.6 & 566.6 & 22.17 & 0.62 & 38 & 11.87 & 44 \\
P-28TD & 79293.5 & 55500.2 & 506.9 & 22.56 & 2.48 & 145 & 9.78 & 4 \\
P-28 TE & 79296.4 & 55514.8 & 416.8 & 20.79 & 0.05 & 73 & 5.9 & 128 \\
& & & & & & & & \\
P-29 A & 86466.5 & 42751.6 & 316.7 & 20.73 & 0.05 & 64 & 6.1 & 106 \\
P-29 B & 86476.1 & 42763.7 & 191.5 & 20.74 & 10.06 & 66 & 5.52 & 409 \\
P-29C & 86485.1 & 42775.4 & 142.3 & 19.51 & 5.42 & 40 & 5.05 & 378 \\
P-29D & 86494.6 & 42787.2 & 114.8 & 20.82 & 7.96 & 41 & 4.96 & 434 \\
P-29TA & 86482.6 & 42796.2 & 692.3 & 20.89 & 5.47 & 30 & 5.31 & 299 \\
P-29TC & 86464.2 & 42773.0 & 512.0 & 20.99 & 3.74 & 50 & 5.92 & 225 \\
P-29TD & 86455.7 & 42761.5 & 431.8 & 20.61 & 1.45 & 47 & 5.33 & 185
\end{tabular}


TABLE 2. Sole carbon sources in Biolog ${ }^{\circledR}$ GN microtiter plates

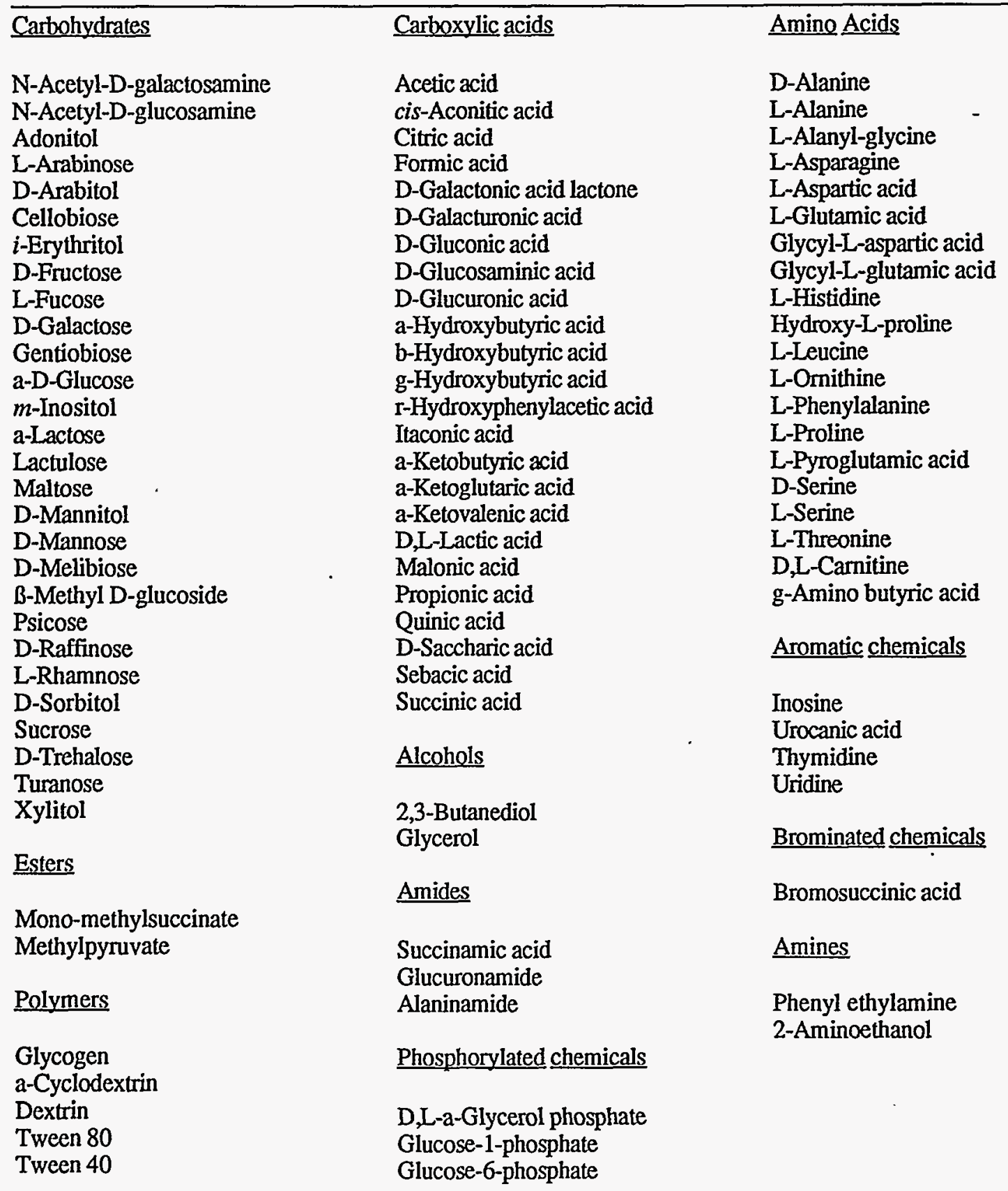



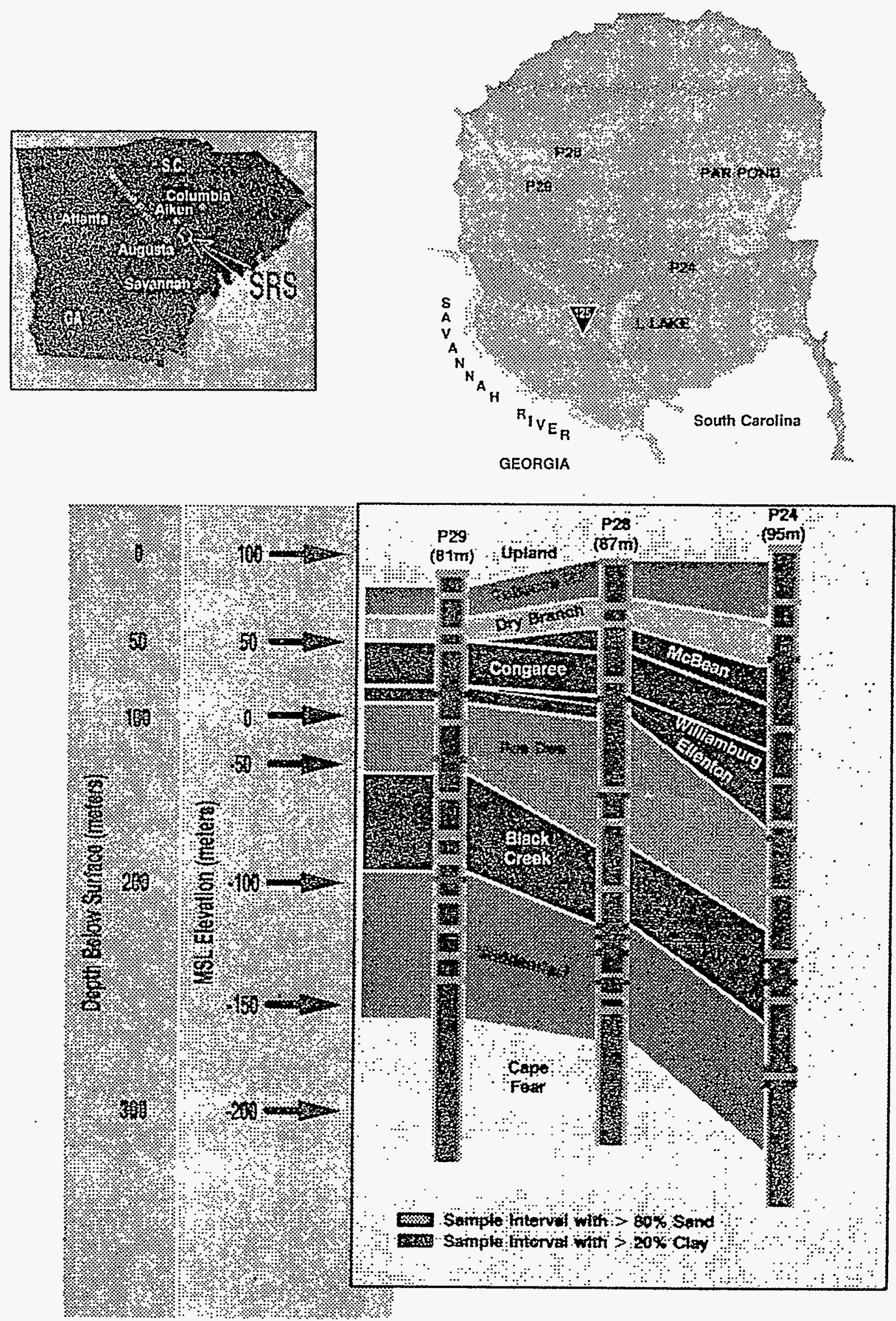

Figure 1. Map of Savannah River Site and Well Clusters 
FIGURE .2. .

Depth (it) By Aquifers

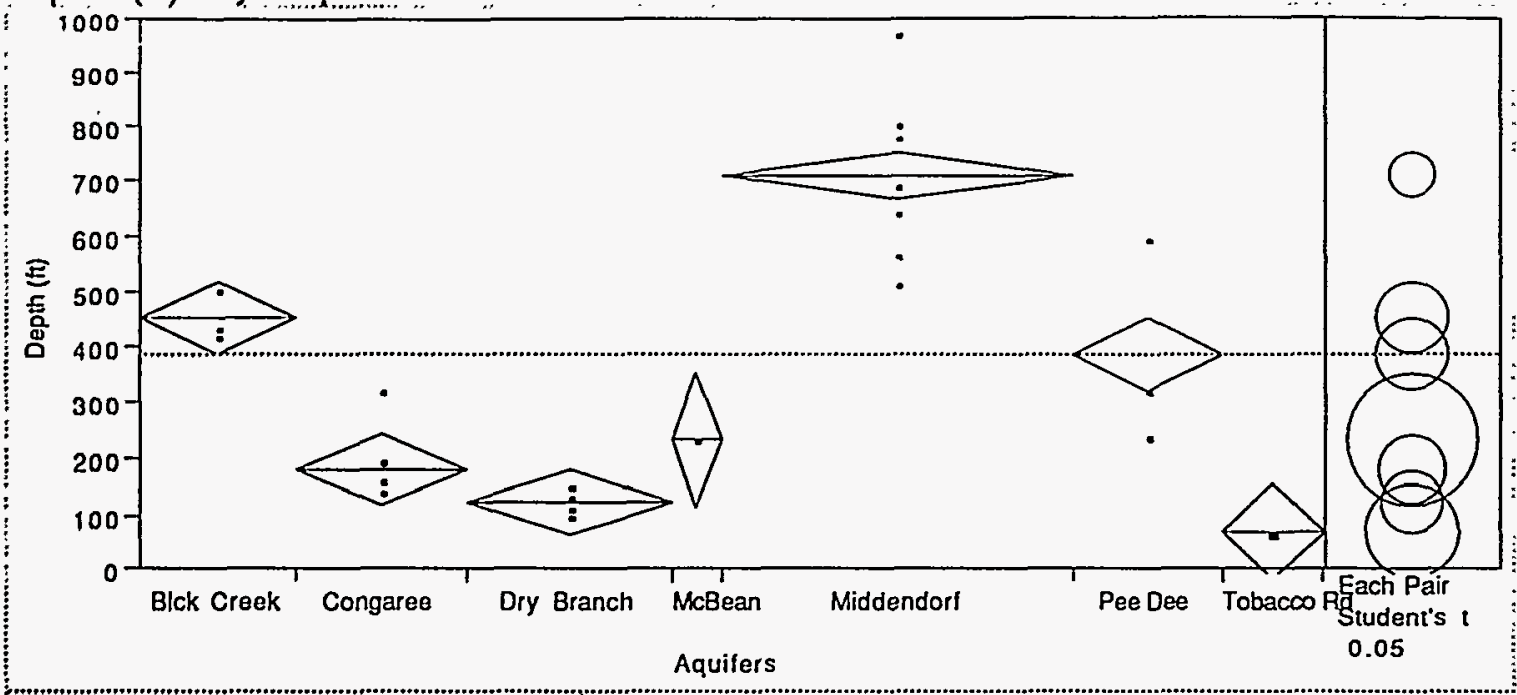

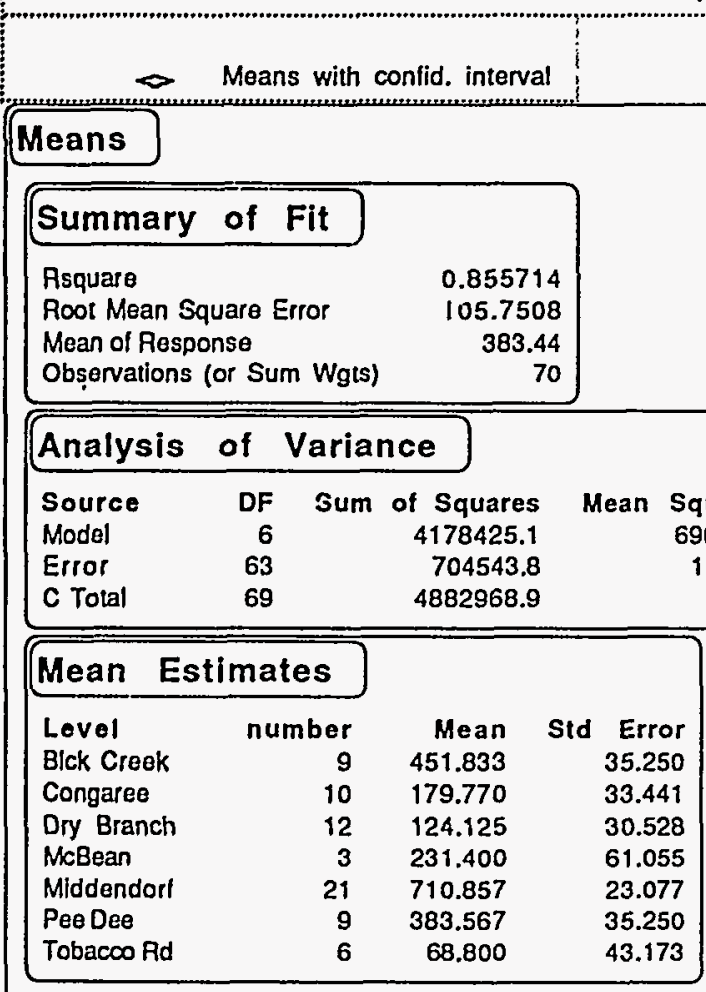

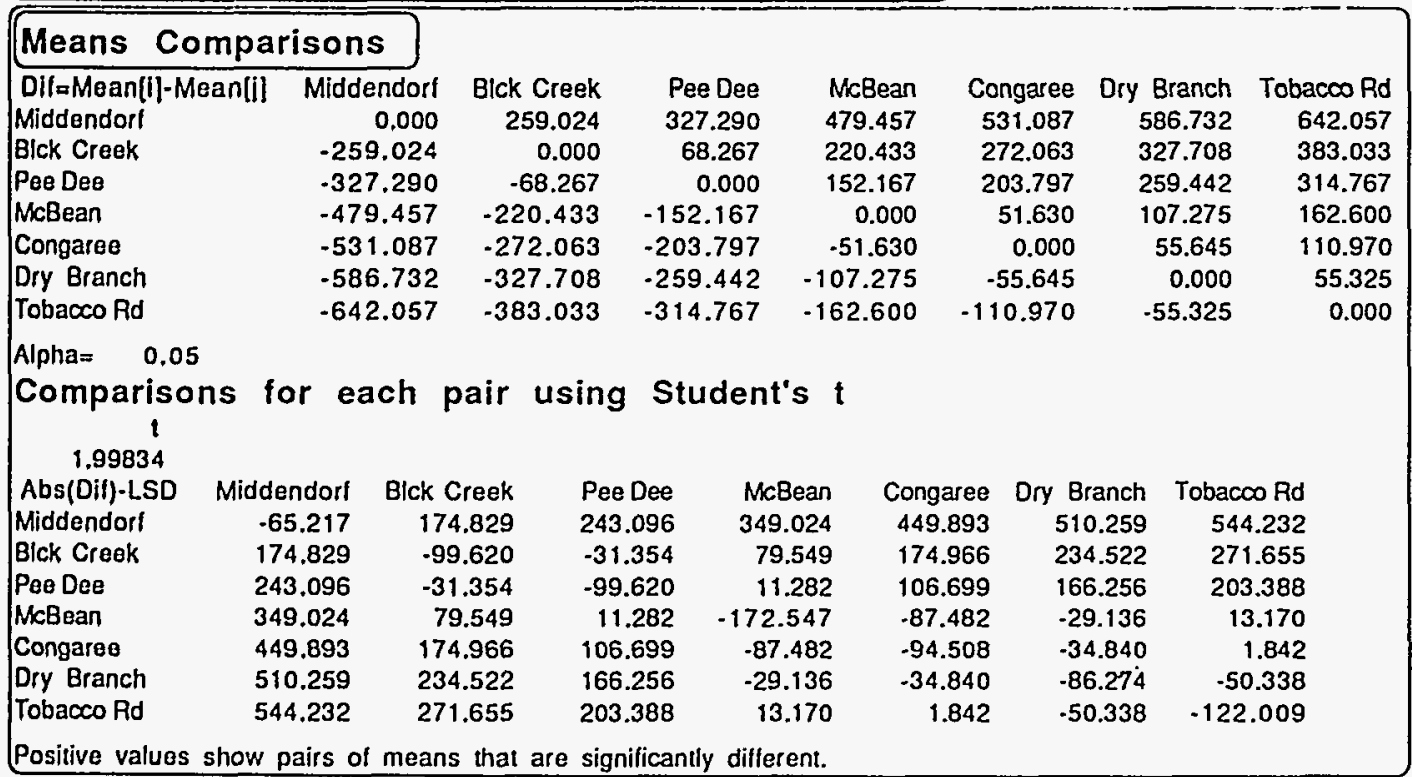


FIGURE 4.

D.O. (ppm) By Aquifers

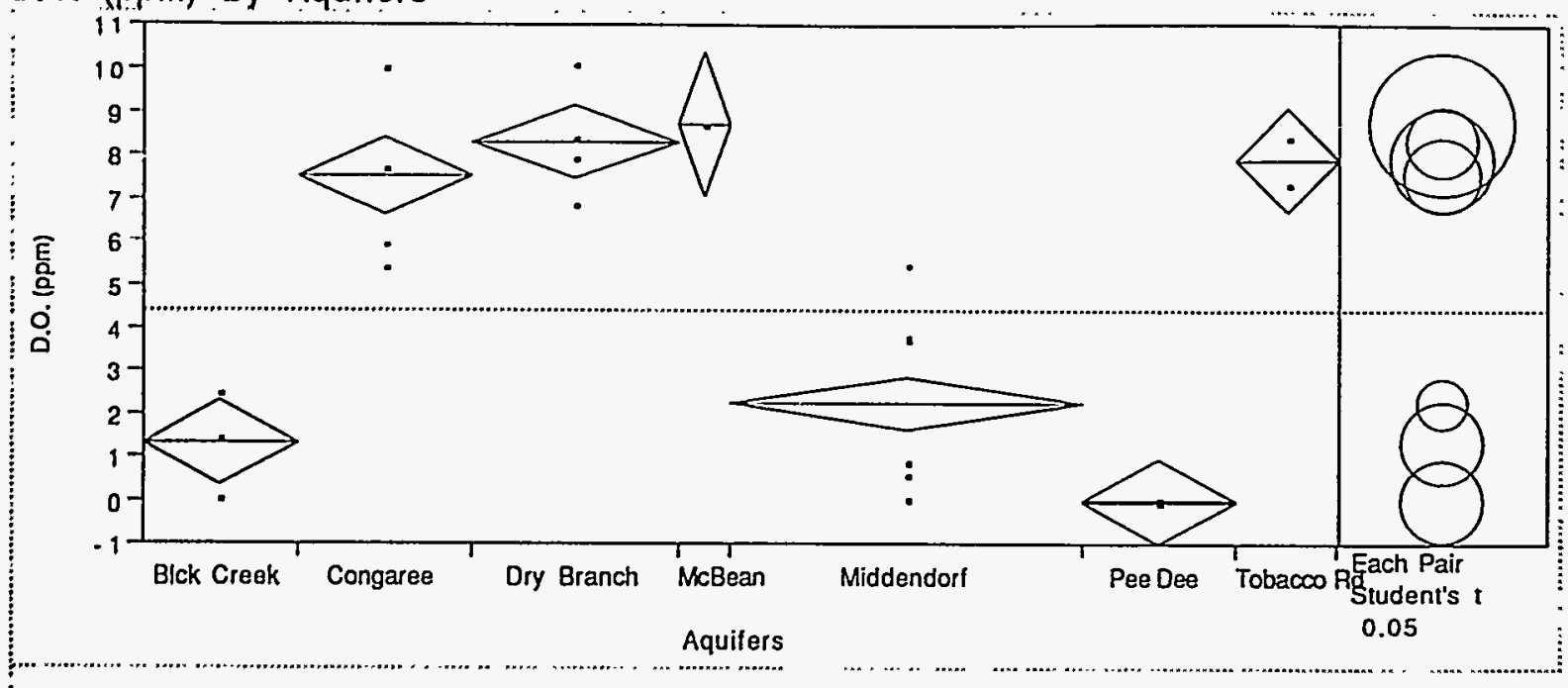

$\infty$ Means with confid. interval

Means

Summary of Fit

Rsquare

Root Mean Square Error

Mean of Response

Observations (or Sum Wgts)

1.491123

4.401857

70

Analysis of Variance

\begin{tabular}{|lrrrrr}
\hline Source & DF & Sum of Squares & Mean Square & F Ratio \\
Model & 6 & 775.29104 & 129.215 & 58.1148 \\
Error & 63 & 140.07721 & 2.223 & Prob $>$ F \\
C Total & 69 & 915.36826 & & & 0.0000 \\
\hline
\end{tabular}

Mean Estimates

Level number

Blck Creek

Congaree

Dry Branch

McBean

Middendorf

Pee Dee

Tobacco Rd

$\begin{array}{rr}\text { number } & \text { Mean } \\ 9 & 1.32667 \\ 10 & 7.56000 \\ 12 & 8.34500 \\ 3 & 8.76000 \\ 21 & 2.21429 \\ 9 & 0.02333 \\ 6 & 7.91000\end{array}$

Std Error

0.49704

0.47153

0.43045

0.86090

0.32539

0.49704

0.60875

\section{Means Comparisons}

$$
\text { Di }
$$

Congaree

Comparisons for each pair using Student's $t$ 1.99834

Abs(Dif)-LSD

McBean

Dry Branch

Tobacco Rd

Congaree

Middendorl

Blck Creek

Pee Des

$\begin{array}{rrr}\text { McBean } & \text { Dry Branch } & \text { Tobacco Ad } \\ -2.43298 & -1.50844 & -1.25702 \\ -1.50844 & -1.21649 & -1.05489 \\ -1.25702 & -1.05489 & -1.72037 \\ -0.76153 & -0.49086 & -1.18875 \\ 4.70656 & 5.05241 & 4.31635 \\ \mathbf{5 . 4 4 6 8 2} & 5.70438 & 5.01285 \\ \mathbf{6 . 7 5 0 1 5} & 7.00771 & 6.31619\end{array}$

Congaree Middendorf Blck Creek $-0.76153$ $-0.49086$

$-1.18875$

$-1.33260$

4.20085

4.86422

6.16755
4.70656

5.05241

4.31635

4.20085

$-0.91958$

$-0.29955$

1.00378
5.44682

5.70438

5.01285

4.86422

$-0.29955$

$-1.40468$

$-0.10135$
Pee Dee

6.75015

7.00771

6.31619

6.16755

1.00378

$-0.10135$

$-1.40468$

Positive values show pairs of means that are significantly different. 
FIGURE 5 .

Conductivity By Aquifers

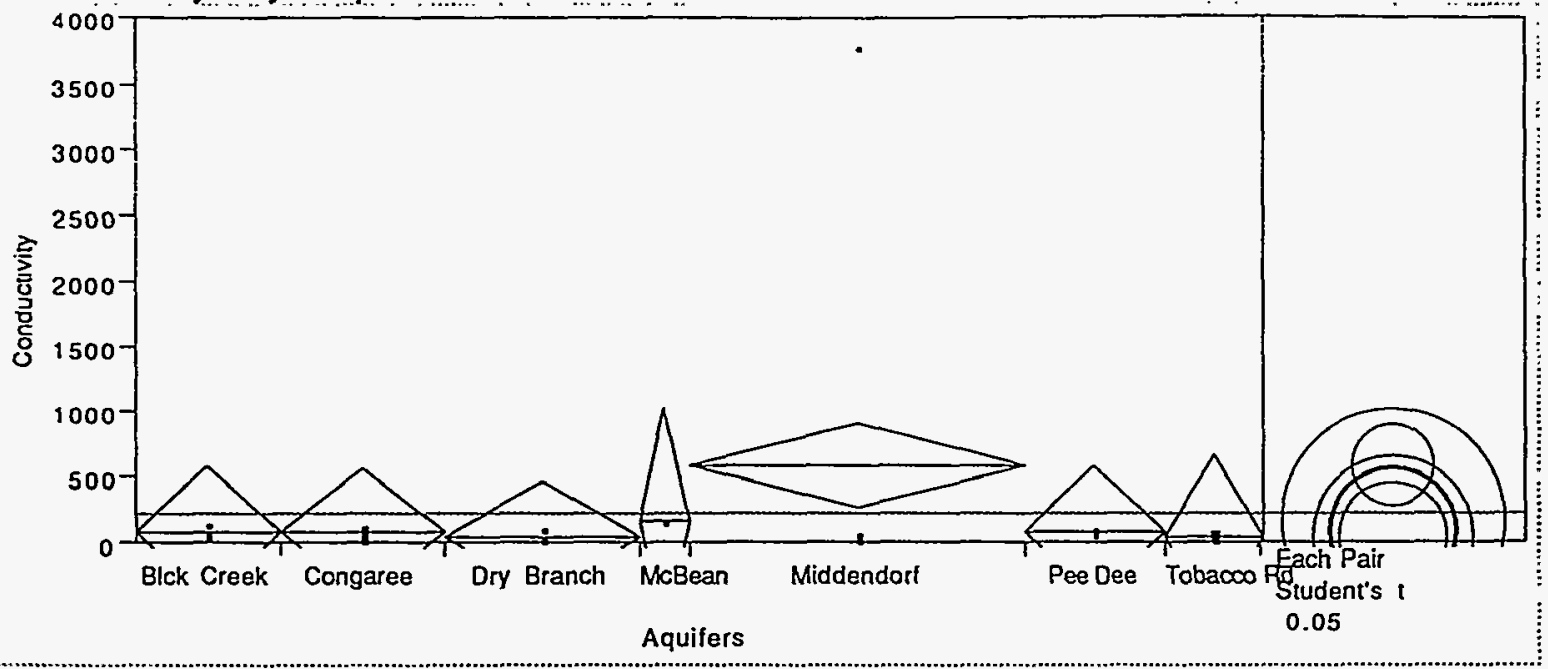

\begin{tabular}{|l|r|}
\hline Means & Means with confid. interval \\
\hline Summary of Fit & \\
Rsquare & $\mathbf{0 . 0 9 6 7 0 9}$ \\
Root Mean Square Error & 757.4831 \\
Mean of Response & 229.7571 \\
Observations (or Sum Wgts) & 70 \\
\hline
\end{tabular}

\begin{tabular}{|lrrrr}
\hline Analysis & of & Variance & & \\
Source & DF & Sum of Squares & Mean Square & F Ratio \\
Model & 6 & 3870153 & 645025 & 1.1242 \\
Error & 63 & 36148182 & 573781 & Prob $>F$ \\
C Total & 69 & 40018335 & & 0.3587 \\
\hline
\end{tabular}

\begin{tabular}{|lrrr|}
\hline Mean Estimates & & \\
Level & number & Mean & Std Error \\
Blck Creek & 9 & 88.333 & 252.49 \\
Congaree & 10 & 80.700 & 239.54 \\
Dry Branch & 12 & 55.000 & 218.67 \\
McBean & 3 & 163.000 & 437.33 \\
Middendorf & 21 & 587.286 & 165.30 \\
Pee Dee & 9 & 80.667 & 252.49 \\
Tobacco Rd & 6 & 45.500 & 309.24 \\
\hline
\end{tabular}

\begin{tabular}{|lrrrrrrrr|}
\hline Means Comparisons & & & & & & \\
\hline Dif=Mean[i]-Mean[j] & Middendorf & McBean & Blck Creek & Congaree & Pee Dee & Ory Branch & Tobacco Rd \\
Middendorf & 0.000 & 424.286 & 498.952 & 506.586 & 506.619 & 532.286 & 541.786 \\
McBean & -424.286 & 0.000 & 74.667 & 82.300 & 82.333 & 108.000 & 117.500 \\
Blck Creek & -498.952 & -74.667 & 0.000 & 7.633 & 7.667 & 33.333 & 42.833 \\
Congaree & -506.586 & -82.300 & -7.633 & 0.000 & 0.033 & 25.700 & 35.200 \\
Pee Dee & -506.619 & -82.333 & -7.667 & -0.033 & 0.000 & 25.667 & 35.167 \\
Dry Branch & -532.286 & -108.000 & -33.333 & -25.700 & -25.667 & 0.000 & 9.500 \\
Tobacco Rd & -541.786 & -117.500 & -42.833 & -35.200 & -35.167 & -9.500 & 0.000
\end{tabular}

Alpha $=0.05$

Comparisons for each pair using Student's $t$ 1.99834

$\begin{array}{lrrrrrrr}\text { Abs(Dif)-LSD } & \text { Middendorf } & \text { McBean } & \text { Blck Creek } & \text { Congaree } & \text { Pee Dee } & \text { Ory Branch } & \text { Tobacco Rd } \\ \text { Middendorf } & -467.14 & -510.00 & -104.12 & -75.00 & -96.46 & -15.49 & -158.93 \\ \text { McBean } & -510.00 & -1235.94 & -934.47 & -914.15 & -926.81 & -869.10 & -952.85 \\ \text { Blck Creek } & -104.12 & -934.47 & -713.57 & -687.87 & -705.90 & -634.15 & -754.96 \\ \text { Congaree } & -75.00 & -914.15 & -687.87 & -676.95 & -695.47 & -622.43 & -746.48 \\ \text { Pee Dee } & -96.46 & -926.81 & -705.90 & -695.47 & -713.57 & -641.82 & -762.63 \\ \text { Dry Branch } & -15.49 & -869.10 & -634.15 & -622.43 & -641.82 & -617.97 & -747.36 \\ \text { Tobacco Rd } & -158.93 & -952.85 & -754.96 & -746.48 & -762.63 & -747.36 & -873.94\end{array}$

$\begin{array}{llllllll}\text { Tobacco Rd } & -158.93 & .952 .85 & .754 .96 & .746 .48 & .762 .63 & .747 .36 & .873 .94\end{array}$

Positive values show pairs of means that are significantly different. 
FIGURE: 6 .

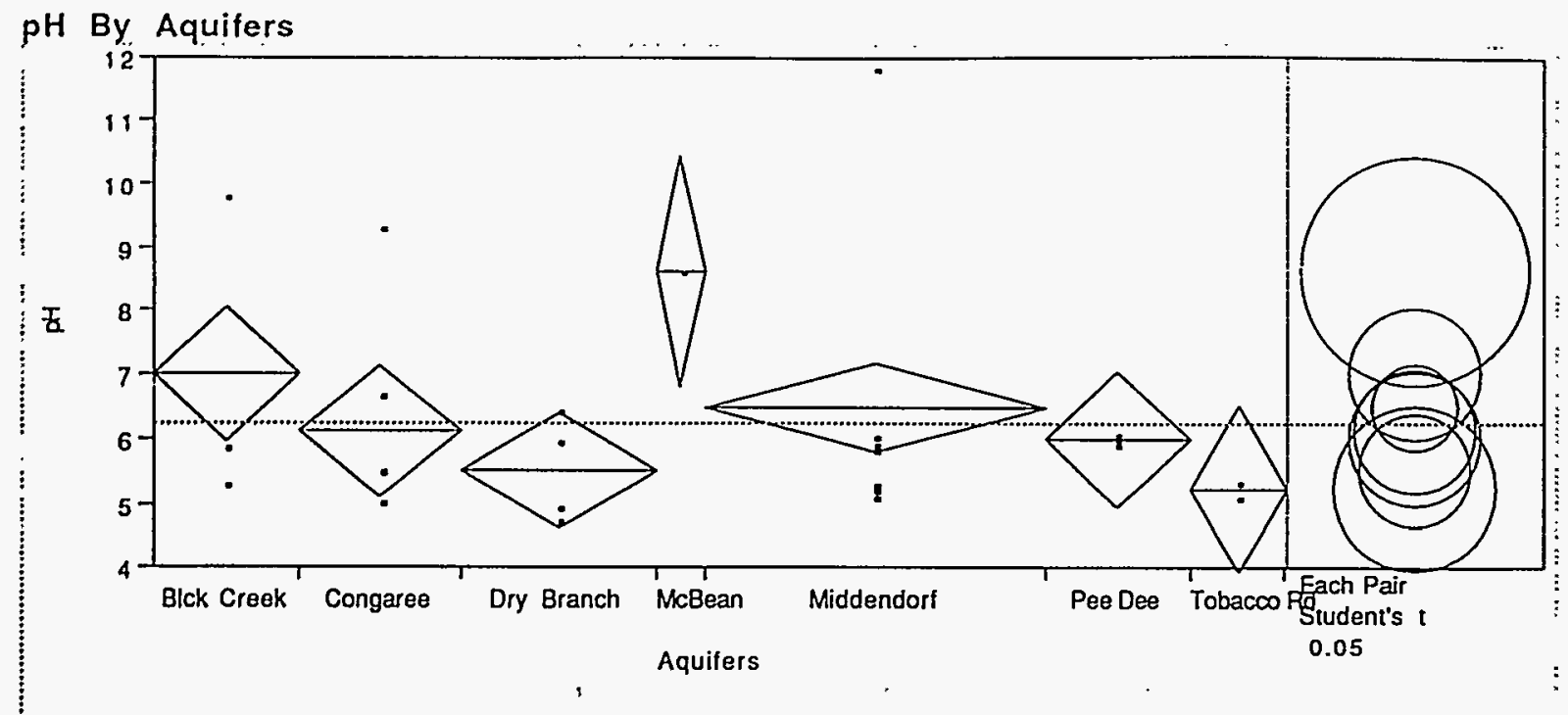

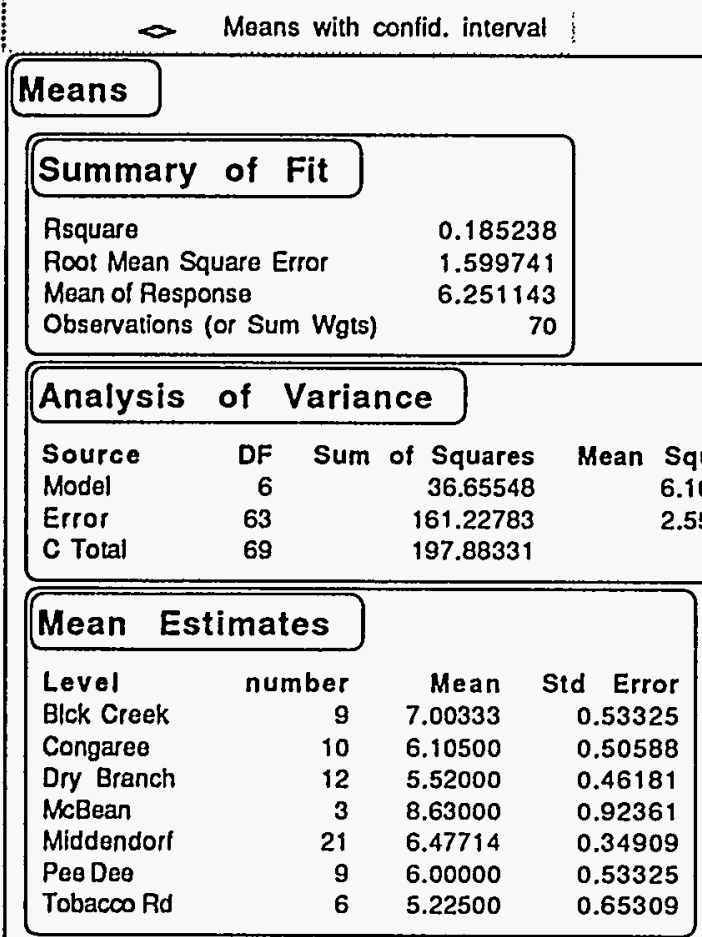

\begin{tabular}{|lrrrrrrr|}
\hline \hline Means Comparisons & & & & & & \\
\hline Dil=Mean[i]-Mean[j] & McBean & Blck Creek & Middendorf & Congaree & Pee Dee & Dry Branch & Tobacco Rd \\
McBean & 0.00000 & 1.62667 & 2.15286 & 2.52500 & 2.63000 & 3.11000 & 3.40500 \\
Bick Creek & -1.62667 & 0.00000 & 0.52619 & 0.89833 & 1.00333 & 1.48333 & 1.77833 \\
Middendorf & -2.15286 & -0.52619 & 0.00000 & 0.37214 & 0.47714 & 0.95714 & 1.25214 \\
Congaree & -2.52500 & -0.89833 & -0.37214 & 0.00000 & 0.10500 & 0.58500 & 0.88000 \\
Pee Dee & -2.63000 & -1.00333 & -0.47714 & -0.10500 & 0.00000 & 0.48000 & 0.77500 \\
Dry Branch & -3.11000 & -1.48333 & -0.95714 & -0.58500 & -0.48000 & 0.00000 & 0.29500 \\
Tobacco Rd & -3.40500 & -1.77833 & -1.25214 & -0.88000 & -0.77500 & -0.29500 & 0.00000 \\
\hline
\end{tabular}

Alpha $=0.05$

Comparisons for each pair using Student's $t$

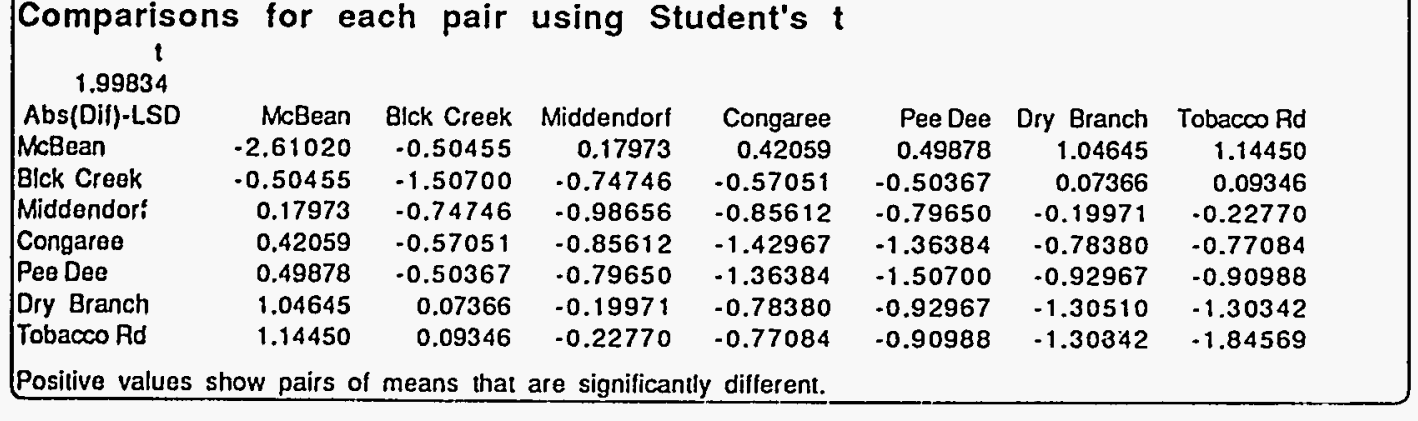


FIGURE 8 .

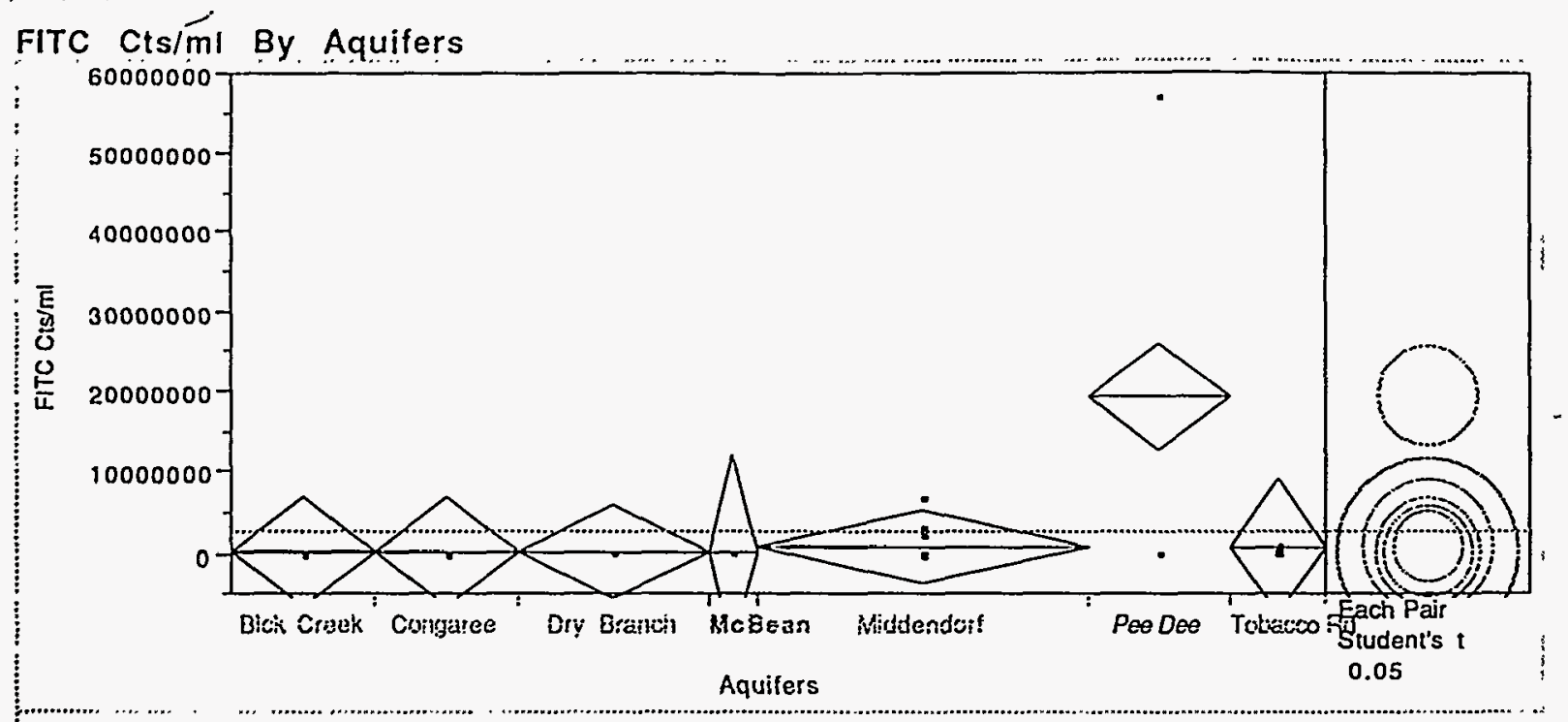

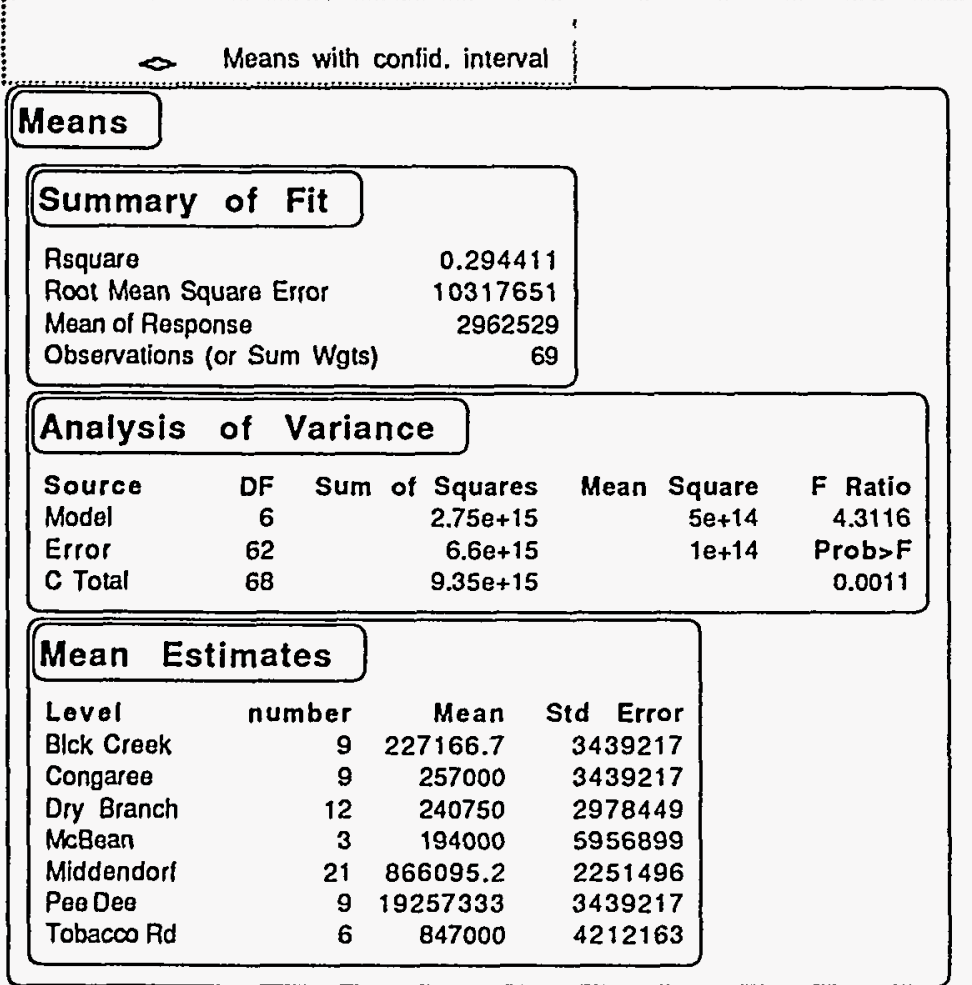

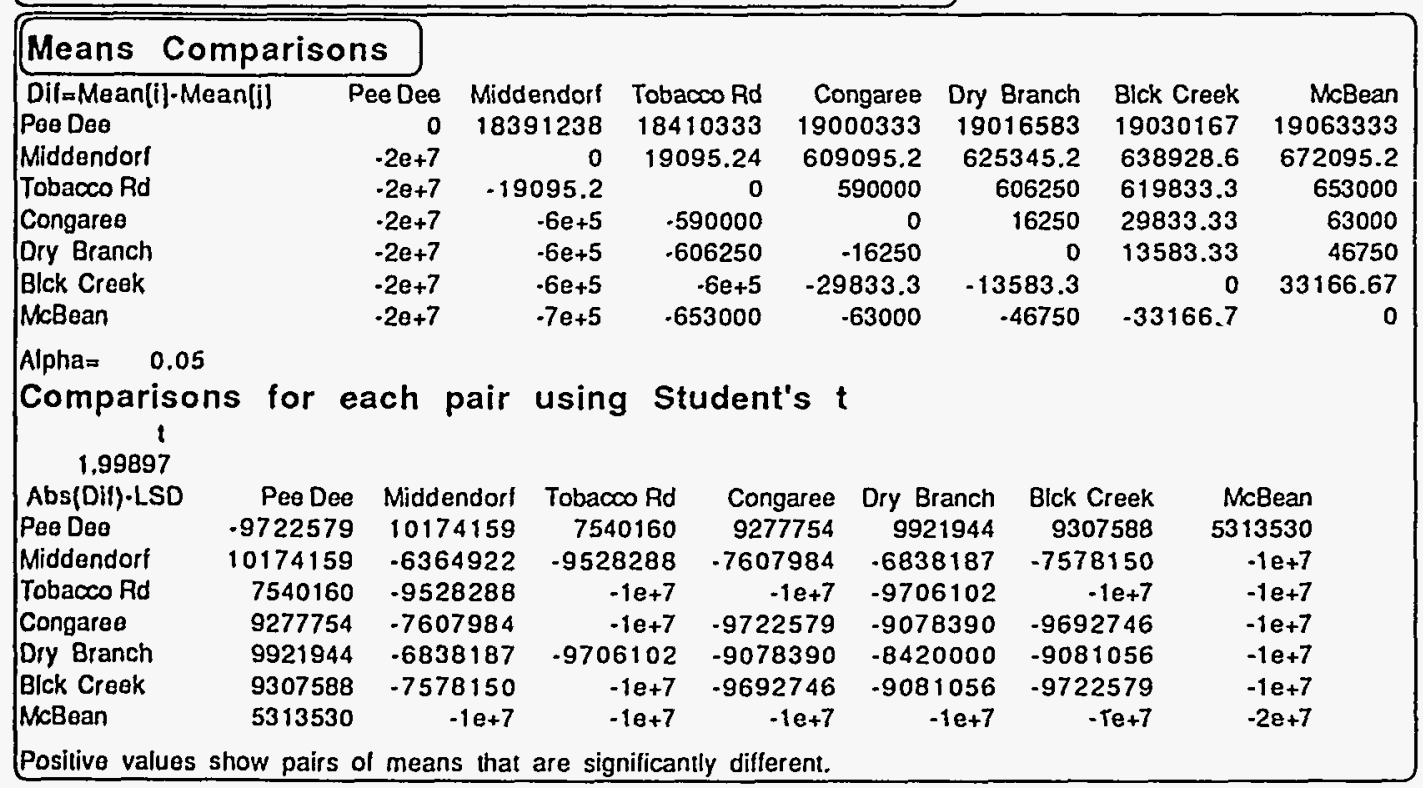


FIGURE 9.

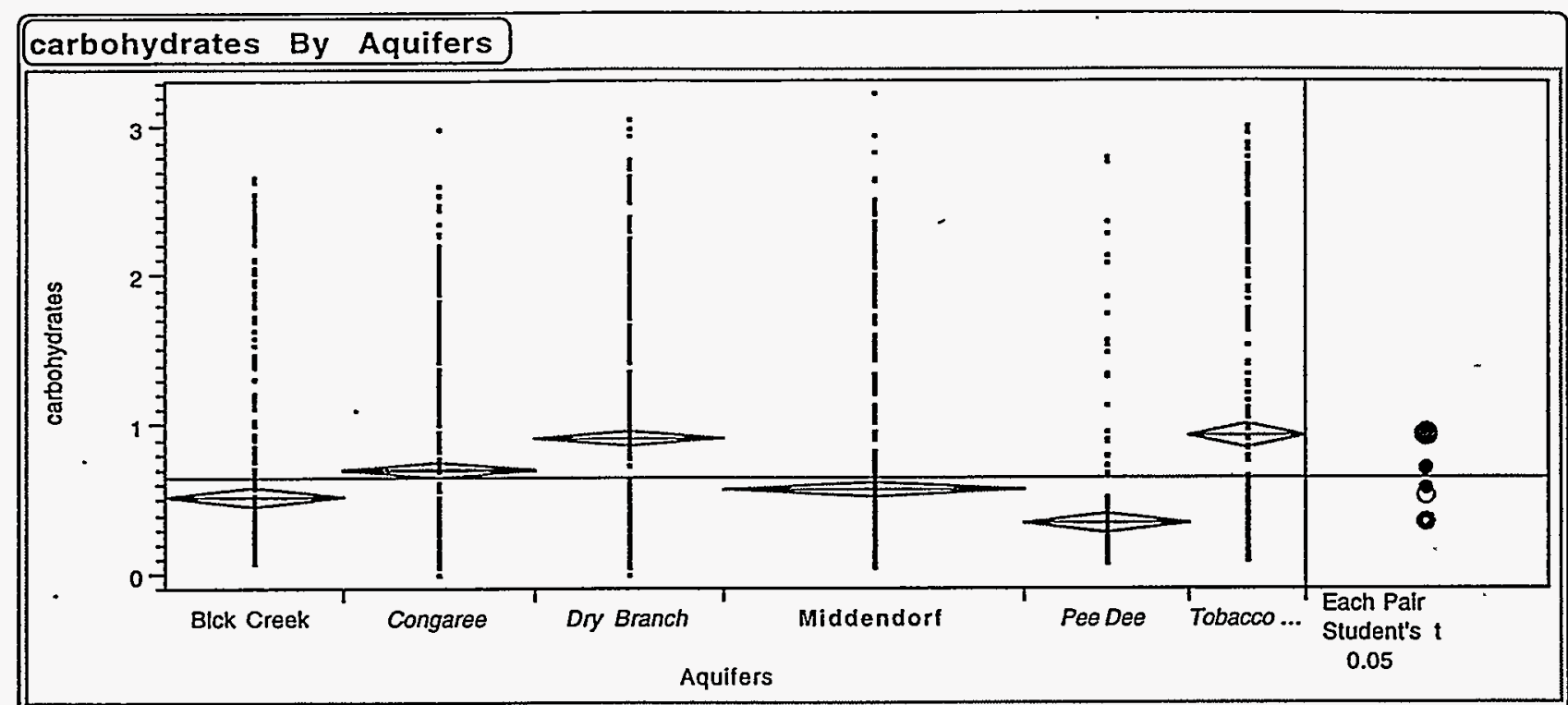

\section{Oneway Anova}

\begin{tabular}{|lr|}
\hline Summary of Fit & \\
RSquare & 0.077045 \\
RSquare Adj & 0.075209 \\
Root Mean Square Error & 0.671515 \\
Mean of Response & 0.643209 \\
Observations (or Sum Wgts) & 2519 \\
\hline
\end{tabular}

\begin{tabular}{|lrrrrr|}
\hline Analysis & of & \multicolumn{2}{c|}{ Variance } & & \\
Source & DF & Sum of Squares & Mean & Square & F Ratio \\
Model & 5 & 94.5957 & 18.9191 & 41.9555 \\
Error & 2513 & 1133.1942 & 0.4509 & Prob $>$ F \\
C Total & 2518 & 1227.7899 & & 0.0000 \\
\hline
\end{tabular}

Means for Oneway Anova

Level Number Mean std Error

$\begin{array}{llll}\text { Blck Creek } & 392 & 0.529033 & 0.03392\end{array}$

4190.693881

$\begin{array}{llll}\text { Dry Branch } & 420 & 0.912086 & 0.03277\end{array}$

McBean $\quad 0$. .

$\begin{array}{llll}\text { Middendorf } & 672 & 0.567479 & 0.02590\end{array}$

$\begin{array}{llll}\text { Pee Dee } & 364 & 0.339445 & 0.03520\end{array}$

$\begin{array}{llll}\text { Tobacco Rd } \quad 252 & 0.929155 & 0.04230\end{array}$

Std Error uses a pooled estimate of error variance

\begin{tabular}{|c|c|c|c|c|c|c|c|}
\hline \multicolumn{8}{|c|}{ Means Comparisons } \\
\hline$D I f=$ Mean $[1]-M e a n[j]$ & Dry Branch & Congaree & Blck Creek & McBean & Tobacco Rd & Middendorf & Pee Dee \\
\hline Dry Branch & 0.000000 & 0.218205 & 0.383053 & 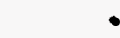 & -0.01707 & 0.344607 & 0.572641 \\
\hline Congaree & -0.21821 & 0.000000 & 0.164848 & - & -0.23527 & 0.126402 & 0.354436 \\
\hline BIck Creek & -0.38305 & -0.16485 & 0.000000 & $\bullet$ & -0.40012 & -0.03845 & 0.189588 \\
\hline McBean & - & 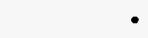 & . & • & - & - & \\
\hline Tobacco Rd & 0.017069 & 0.235274 & 0.400122 & $\bullet$ & 0.000000 & 0.361676 & 0.589710 \\
\hline Middendorf & -0.34461 & -0.1264 & 0.038446 & $\cdot$ & -0.36168 & 0.000000 & 0.228034 \\
\hline Pee Dee & -0.57264 & -0.35444 & -0.18959 & - & -0.58971 & -0.22803 & 0.000000 \\
\hline
\end{tabular}

Alpha $=0.05$

Comparisons for each pair using Student's t

1.96094

Abs(Dif)-LSD

Dry Branch

Congaree

Blck Creek

MicBean

Tobacco Rd

Middendorf

Pee Dee

Dry Branch

Congaree

0.127283

0.290576

0.127283

$-0.09098$

0.072318

$-0.08786 \quad 0.130302$

$0.262699 \quad 0.044434$

0.478342

0.260085
Blck Creek 0.290576

0.072318

$-0.09406$

0.293800

$-0.04524$

0.093739
McBean Tobacco Rd

-0.08786
$-\quad 0.130302$

0.293800

0.044434
-0.04524

0.264407

$-0.07184 \quad 0.142337$

$0.142337-0.09761$

Positive values show pairs of means that are significantly different. 
FIGURE $9 \mathrm{a}$.

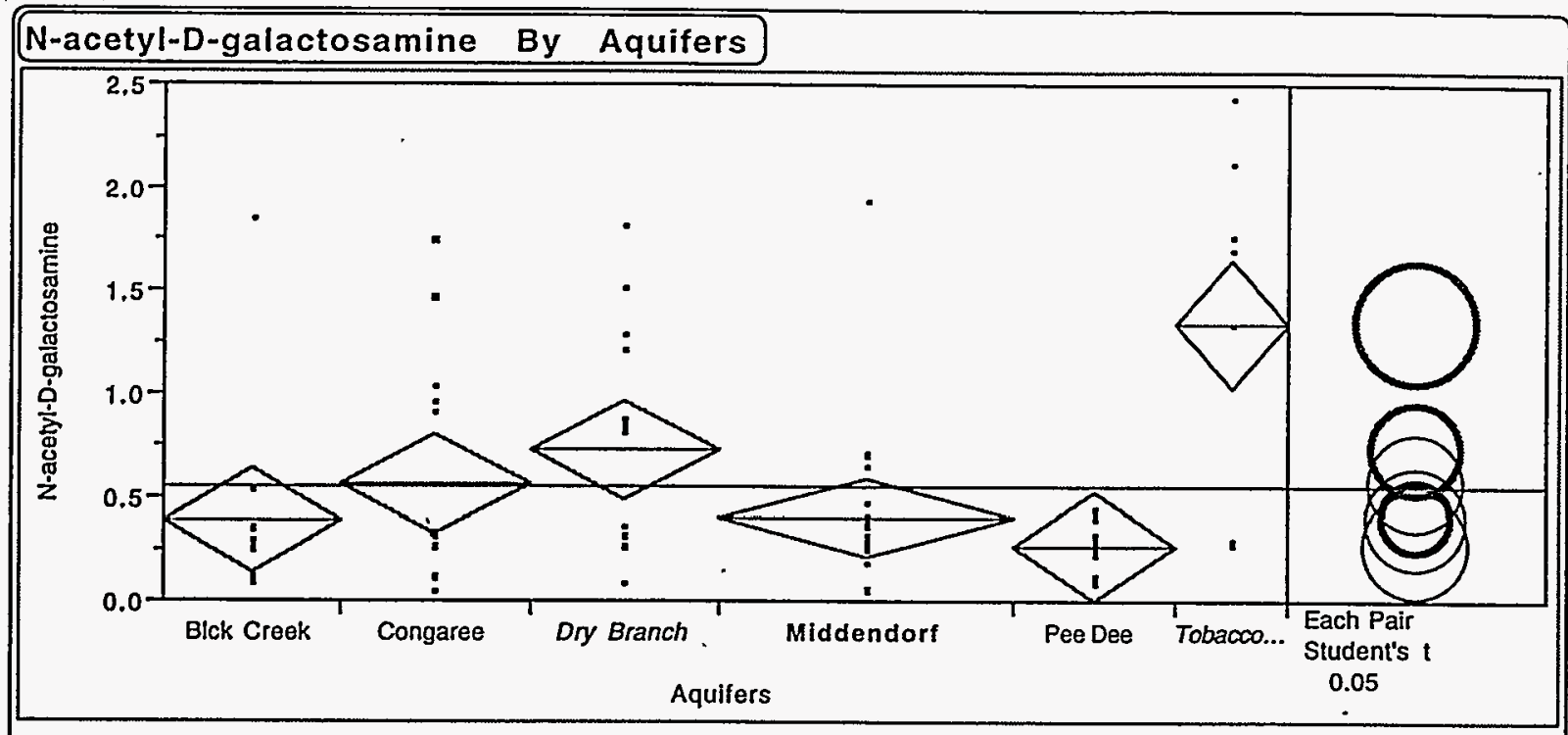

\section{Oneway Anova}

\begin{tabular}{|lr|}
\hline Summary of Fit & \\
RSquare & 0.292128 \\
RSquare Adj & 0.249993 \\
Root Mean Square Error & 0.479891 \\
Mean of Response & 0.553867 \\
Observations (or Sum Wgts) & 90 \\
\hline
\end{tabular}

\begin{tabular}{||lrrrrr|}
\hline Analysis & of & Variance & & \\
Source & DF & Sum of Squares & Mean & Square & F Ratio \\
Model & 5 & 7.983328 & 1.59667 & 6.9331 \\
Error & 84 & 19.344836 & 0.23030 & Prob $>\mathrm{F}$ \\
C Total & 89 & 27.328164 & & 0.0000 \\
\hline
\end{tabular}

Means for Oneway Anova

$\begin{array}{lrrr}\text { Lovel } & \text { Number } & \text { Mean } & \text { Std Error } \\ \text { Blck Creek } & 14 & 0.38971 & 0.12826 \\ \text { Congaree } & 15 & 0.56053 & 0.12391 \\ \text { Dry Branch } & 15 & 0.73020 & 0.12391 \\ \text { McBean } & 0 & & \\ \text { Middendorf } & 24 & 0.39887 & 0.09796 \\ \text { Pee Dee } & 13 & 0.26538 & 0.13310 \\ \text { Tobacco Rd } & 9 & 1.33422 & 0.15996 \\ \text { Std Error uses a pooled estimate of error variance }\end{array}$

Std Error uses a pooled estimate of error variance

\section{Means. Comparisons}

\begin{tabular}{|c|c|c|c|c|}
\hline DIf =Mean[I]-Mean[\}] & Dry & Branch & Congaree & Blck Creek \\
\hline Dry Branch & & 0.00000 & 0.16967 & 0.34049 \\
\hline Congaree & & -0.16967 & 0.00000 & 0.17082 \\
\hline Blck Creek & & -0.34049 & -0.17082 & 0.00000 \\
\hline McBean & & $\bullet$ & 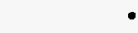 & \\
\hline Tobacco Rd & & 0.60402 & 0.77369 & 0.9445 \\
\hline Middendorf & & -0.33133 & -0.16166 & 0.0091 \\
\hline Pee Deo & & -0.46482 & -0.29515 & -0.1243 \\
\hline
\end{tabular}

$$
\begin{array}{rrrr}
\text { McBean Tobacco Rd } & \text { Middendorf } & \text { Pee Dee } \\
\text { - } & -0.60402 & 0.33133 & 0.46482 \\
\text { - } & -0.77369 & 0.16166 & 0.29515 \\
\text { - } & -0.94451 & -0.00916 & 0.12433 \\
\text { - } & 0.00000 & 0.93535 & 1.06884 \\
\text { - } & -0.93535 & 0.00000 & 0.13349 \\
& -1.06884 & -0.13349 & 0.00000
\end{array}
$$

Comparisons for each pair using Student's $t$

1.98862

Abs(Dif)-LSD

Dry Branch

Congaree

Blck Creek

McBean

Tobacco Rd

MIddendorf

Peo Deo

Dry Branch

$$
-0.34847
$$

$-0.1788$

$-0.01415$

0.201645

0.017220

0.103192
Blck Creek

$-0.01415$

$-0.18382$

$-0.3607$

0.536778

$-0.31177$

$-0.24324$
McBean Tobacco Rd Middendorf

$0.017220 \quad 0.103192$

$-0.15245 \quad-0.06647$

$-0.31177 \quad-0.24324$

0.5623340 .655016

$-0.27549-0.19515$

$-0.19515-0.37432$

Posiltive values show pairs of means that are significantly different. 
FIGURE 9b.

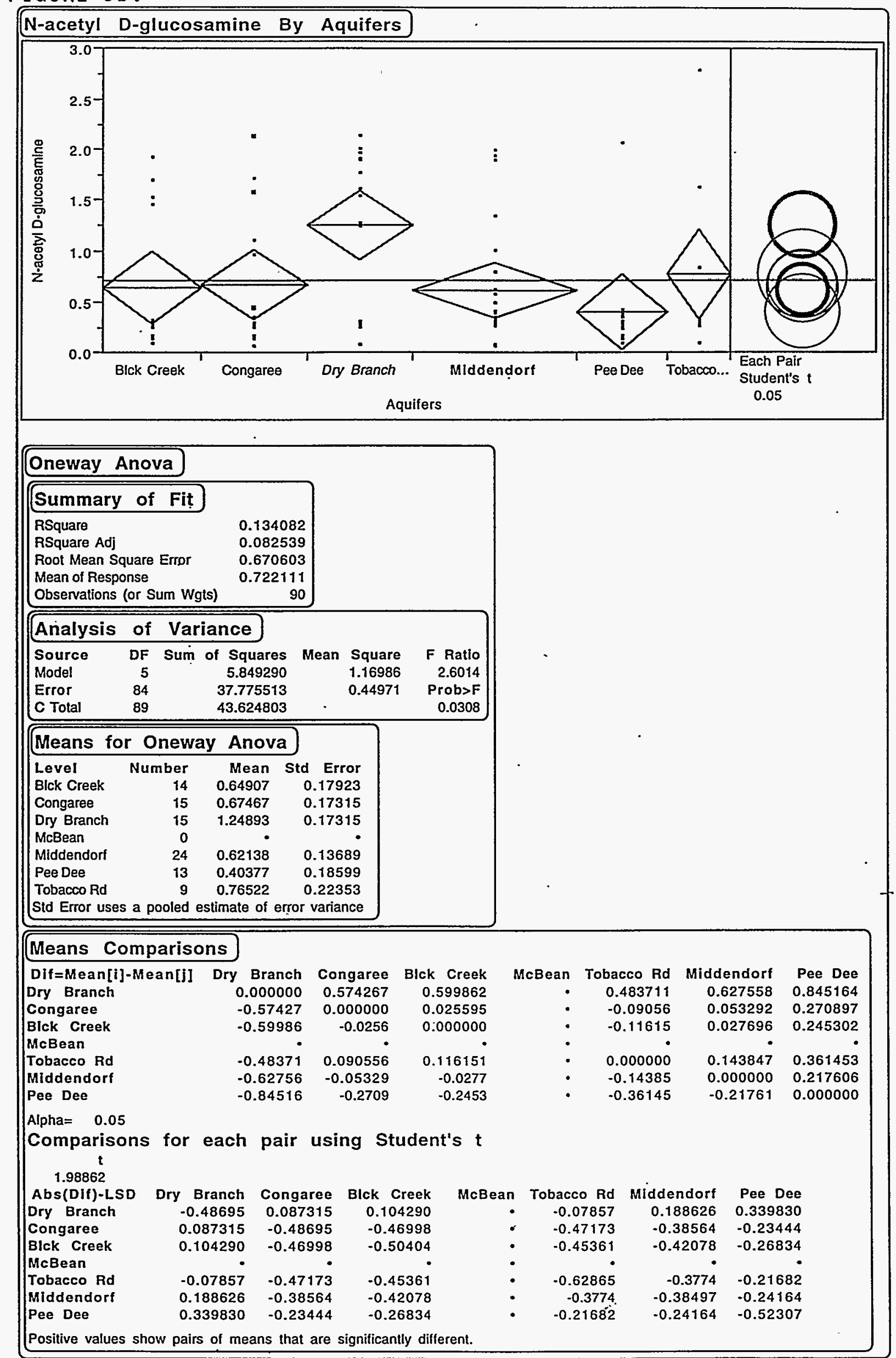


FIGURE -9y

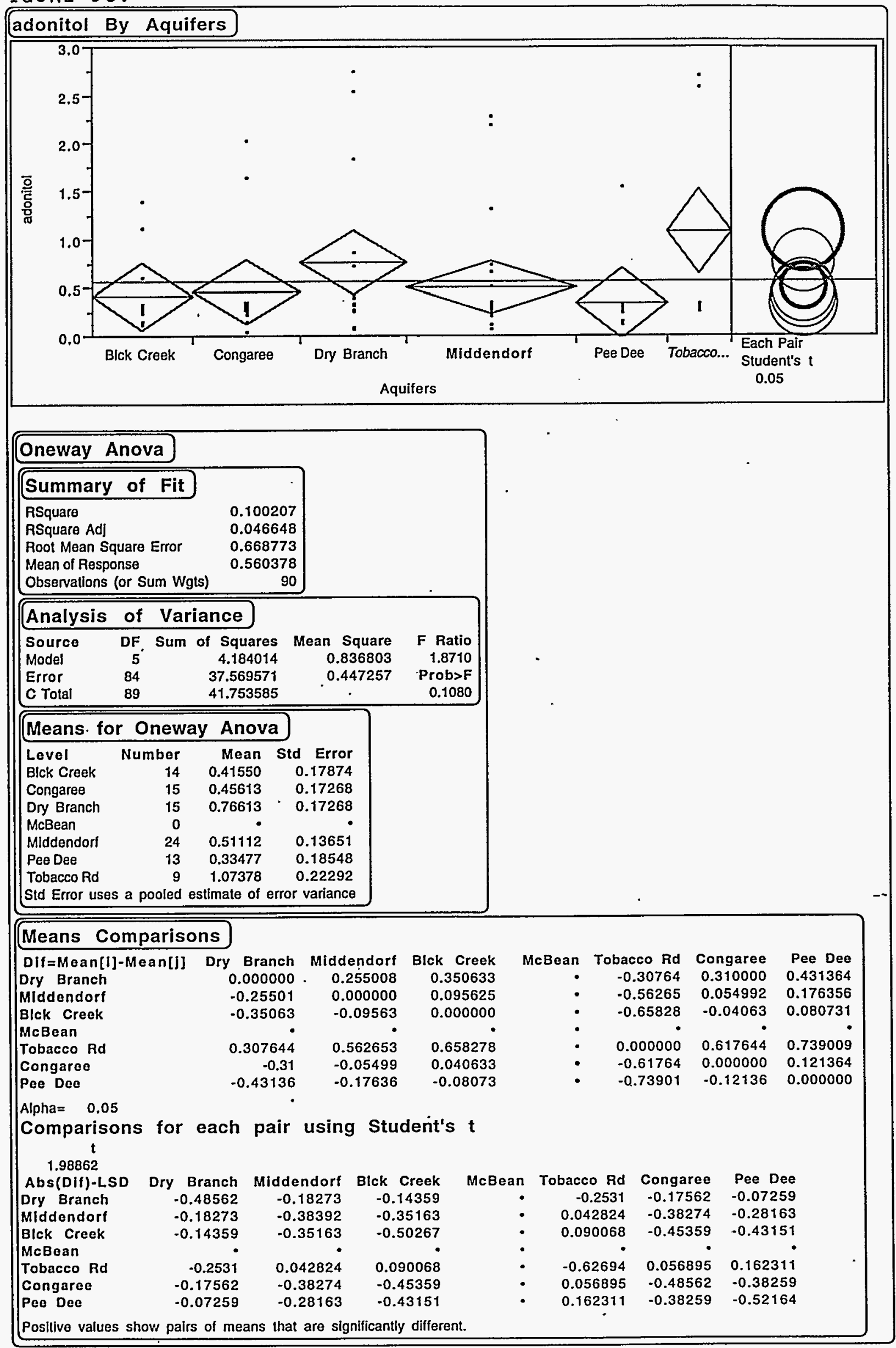



FIGURÉ $9 \mathrm{~d}$.

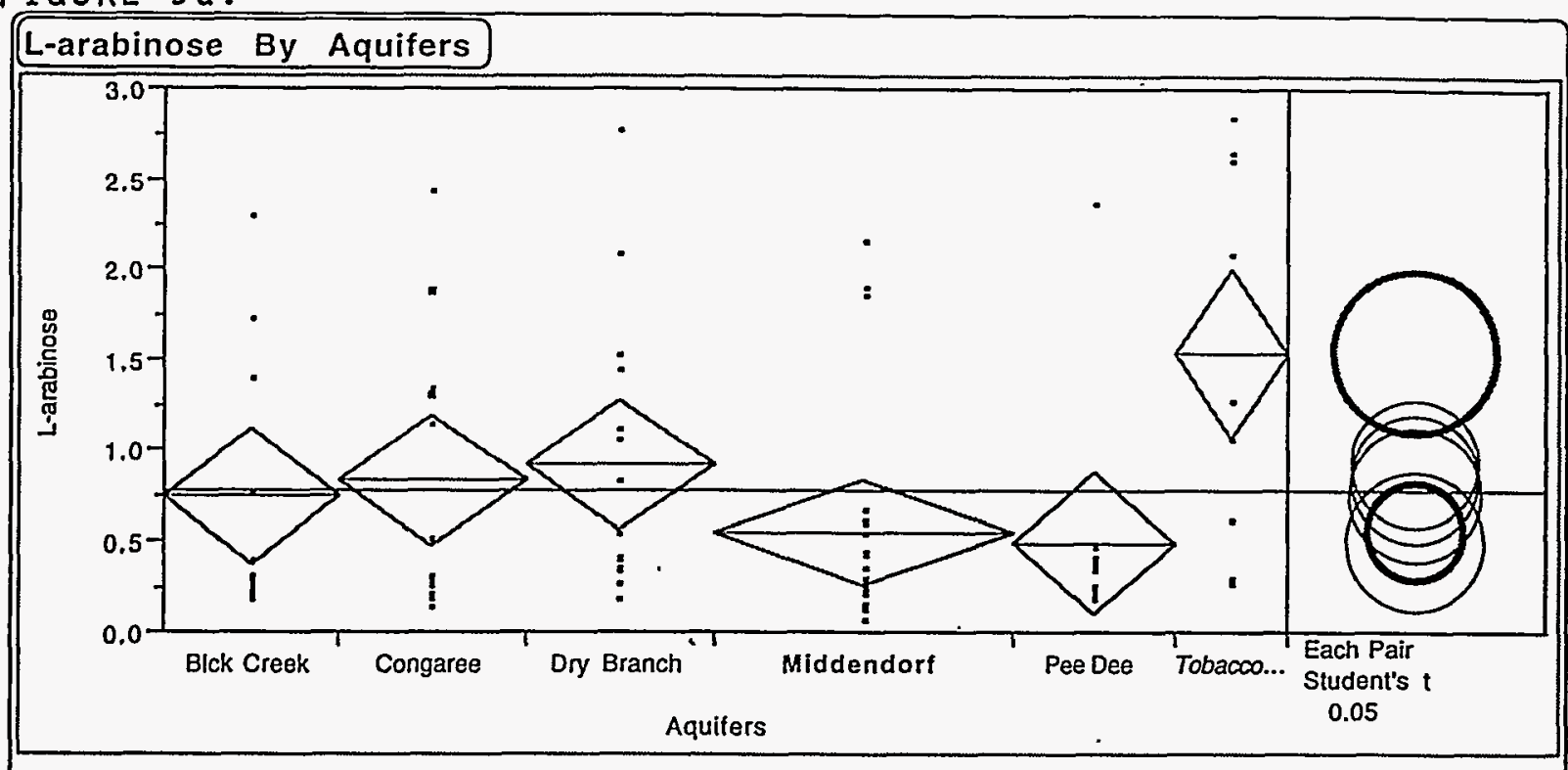

Oneway Anova

Summary of Fit

RSquare

RSquare Ad] .

Root Mean Square Error

Mean of Response

Observations (or Sum Wgts)

0.102041

0.71601

0.776767

\begin{tabular}{|lrrrrr|}
\hline Analysis & of & Variance & & & \\
Source & DF & Sum of Squares & Mean Square & F Ratio \\
Model & 5 & 7.748296 & 1.54966 & 3.0227 \\
Error & 84 & 43.064306 & 0.51267 & Prob>F \\
C Total & 89 & 50.812602 & & 0.0147 \\
\hline
\end{tabular}

Means for Oneway Anova

Level Number Mean Std Error

$\begin{array}{llll}\text { Bick Creek } & 14 & 0.74571 & 0.19136\end{array}$

$\begin{array}{llll}\text { Congaree }^{*} & 15 & 0.83340 & 0.18487\end{array}$

$\begin{array}{llll}\text { Dry Branch } & 15 & 0.91713 & 0.18487\end{array}$

McBean

Middendorf

Pee Deo

$\begin{array}{rr}0 & \bullet\end{array}$

0.14615

Tobacco Rd

$13 \quad 0.49200$

0.19859

Std Error uses a pooled estimat

\section{Means Comparisons}

\section{Dlf=Mean[i]-Mean[i] Dry}

Dry Branch

Congaroe

Blck Creek

McBean

Tobacco Rd

Middendorf

Peo Dee

Branch 0.00000

$-0.08373$

$-0.17142$

0.60864

$-0.37201$

$-0.42513$
Congaree 0.08373

0.00000

$-0.08769$

0.69238

$-0.28828$

$-0.34140$
Blck Creek

0.17142

0.08769

0.00000

0.78006

$-0.20059$

$-0.25371$
McBean Tobacco Rd

$-0.60864$

$-0.69238$

$-0.78006$

0.00000

$-0.98065$

$-1.03378$
Middendorf 0.37201

0.28828

0.20059

0.98065

0.00000

$-0.053 i 2$
Pee Dee 0.42513 0.34140 0.25371

1.03378

0.05312

0.00000

Alpha $=0.05$

Comparisons for each pair using Student's $t$

1.98862

Abs(Dif)-LSD Dry Branch Congaree Blck Creek

Dry Branch

Cóngaree

Blok Creek

McBoan

Tobacco Rd

Middendorf

Peo Deo

$-0.51992$

$-0.43619$

$-0.35771$

$-0.43619$

$-0.51992$

$-0.44144$

$-0.35771$

$-0.44144$

$-0.53817$

0.008288

$-0.09664$

$-0.11442$

0.092021

$-0.18038$

$-0.19815$

0.171720

$-0.27825$

$-0.29471$
McBean Tobacco Rd Middendorf $-0.09664$

$-0.18038$

$-0.27825$

0.092021

0.171720

$-0.67122$

0.424107

0.416346

0.424107

$-0.41104$

$-0.43721$

Pee Dee

$-0.11442$

$-0.19815$

$-0.29471$

0.416346

$-0.43721$

$-0.55849$

Positive values show pairs of means that are significantly different. 
FIGURE $9 f$.

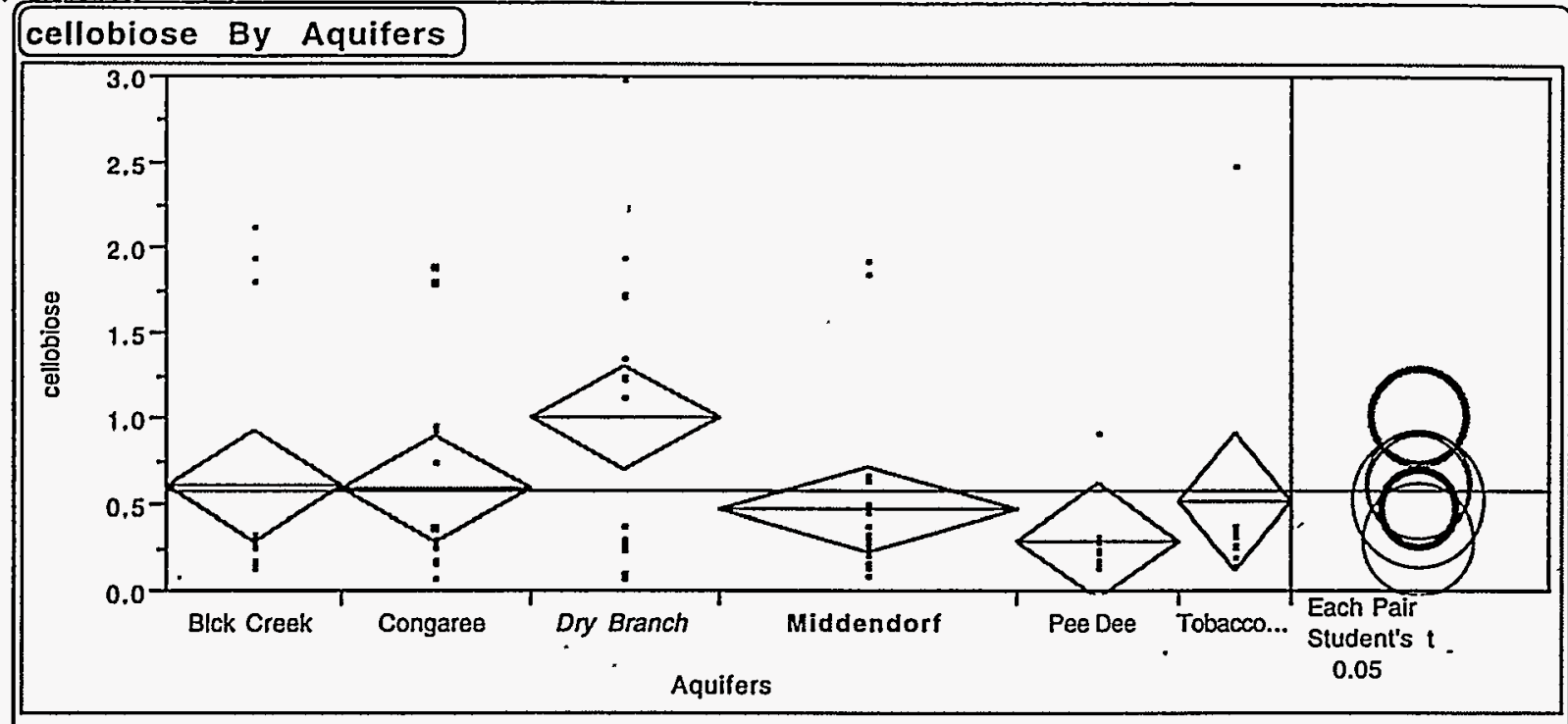

\section{Oneway Anova}

\begin{tabular}{|lr|}
\hline Summary of Fit & \\
RSquare & 0.114914 \\
RSquare Adj & 0.06223 \\
Root Mean Square Error & 0.610247 \\
Mean of Response & 0.58183 \\
Observations (or Sum Wgts) & 90 \\
\hline
\end{tabular}

Analysis of Variance

\begin{tabular}{|lrrrrr} 
Source & DF & Sum of Squares & Mean Square & F Ratio \\
Model & 5 & 4.061402 & 0.812280 & 2.1812 \\
Error & 84 & 31.281715 & 0.372401 & Prob $>$ F \\
C Total & 89 & 35.343116 & & 0.0638 \\
\hline
\end{tabular}

\begin{tabular}{|lrrrr|}
\hline \multicolumn{3}{|c}{ Means for } & Oneway & \multicolumn{2}{c|}{ Anova } & \\
Level & Number & Mean & Std & Error \\
Blck Creek & 14 & 0.61529 & 0.16310 \\
Congaree & 15 & 0.58853 & 0.15757 \\
Dry Branch & 15 & 1.00393 & 0.15757 \\
McBean & 0 & - & \\
Middendorf & 24 & 0.46808 & 0.12457 \\
Pee Dee & 13 & 0.29985 & 0.16925 \\
Tobacco Rd & 9 & 0.52578 & 0.20342 \\
Std Error uses a pooled estimate of error variance &
\end{tabular}

Means Comparisons

\begin{tabular}{|c|c|c|c|c|c|c|c|}
\hline Dif=Mean[I]-Mean[J] & Dry Branch & Blck Creek & Congaree & McBean & Tobacco Rd & Middendorf & Pee Dee \\
\hline Dry Branch & 0.000000 & 0.388648 & 0.415400 & - & 0.478156 & 0.535850 & 0.704087 \\
\hline Blck Creek & -0.38865 & 0.000000 & 0.026752 & $\bullet$ & 0.089508 & 0.147202 & 0.315440 \\
\hline Congaree & -0.4154 & -0.02675 & 0.000000 & - & 0.062756 & 0.120450 & 0.288687 \\
\hline McBean & - & - & - & - & - & - & - \\
\hline Tobacco Rd & -0.47816 & -0.08951 & -0.06276 & - & 0.000000 & 0.057694 & 0.225932 \\
\hline MIddendorf & -0.53585 & -0.1472 & -0.12045 & - & -0.05769 & 0.000000 & 0.168237 \\
\hline Peo Dee & -0.70409 & -0.31544 & -0.28869 & - & -0.22593 & -0.16824 & 0.000000 \\
\hline
\end{tabular}

Alpha $=0.05$

Comparisons for each pair using Student's $t$

1.98862

Abs(Dif)-LSD

Dry Branch

Blck Creek

Dry Branch

Branch Blck Creek

Congaree

$-0.44313$

$-0.06232$

Congaree

McBean

Tobacco Rd

Middendorf

.0 .02773

$-0.06232$

$-0.45868$

$-0.02773$

-0.42422
-0.44313

$-0.03352$

0.136422

Pee Dee

0.244235

$-0.42898 \quad-0.44892$

$-0.26091$

$-0.15198$

$-0.27898$

McBean Tobacco Rd Middendorf

\begin{tabular}{rrrr} 
- Tobacco Rd & Middendorf & Pee Dee \\
\hline & -0.03352 & 0.136422 & 0.244235 \\
& -0.42898 & -0.26091 & -0.15198
\end{tabular}

Positive values show pairs of means that are significantly different. 


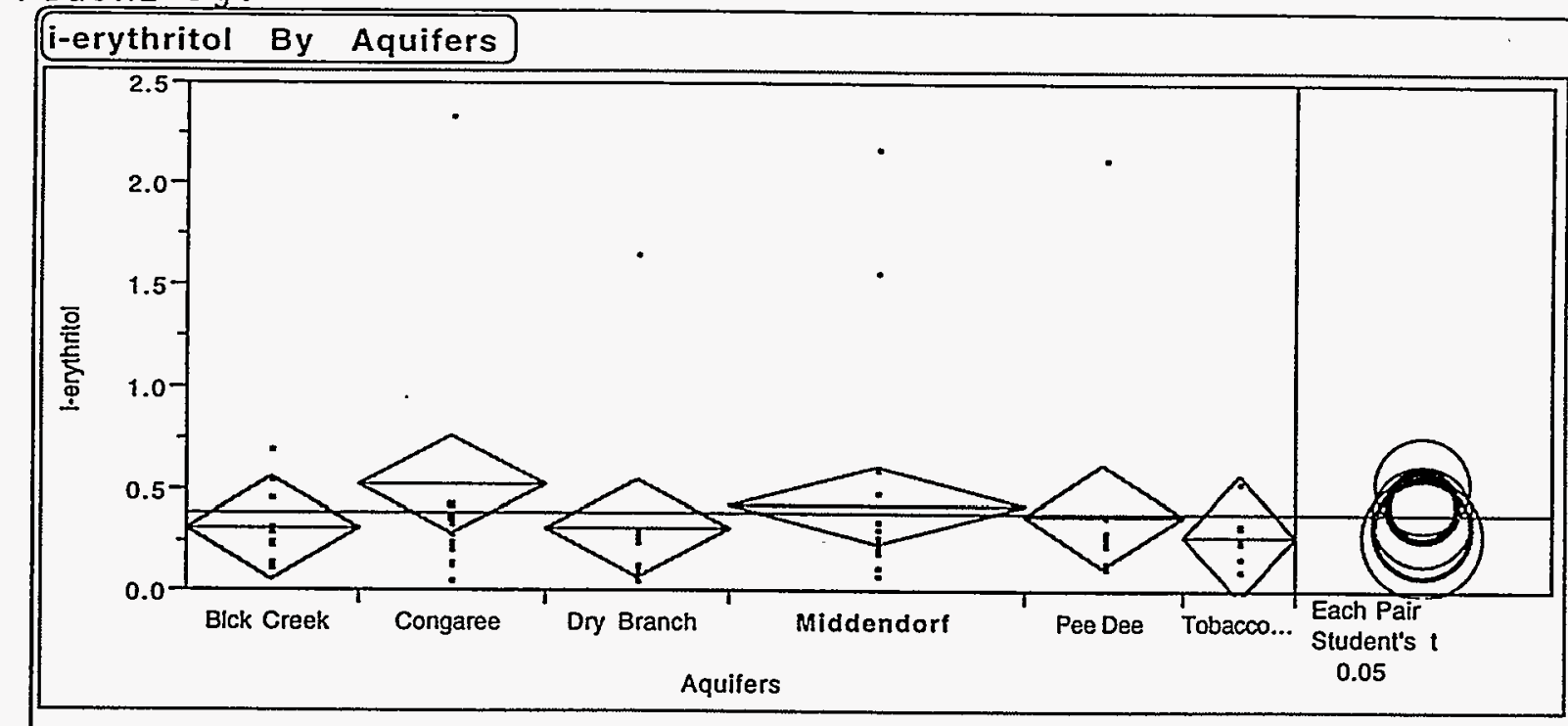

Oneway Anova

\begin{tabular}{|lr|}
\hline Summary of Fit & \\
RSquare & \\
RSquare Adj & 0.031503 \\
Root Mean Square Error & 0.02615 \\
Mean of Response & 0.473282 \\
Observations (or Sum Wgts) & 0.3805 \\
\hline
\end{tabular}

Analysis of Variance

\begin{tabular}{|lrrrrr} 
Source & DF & Sum of Squares & Mean Square & F Ratio \\
Model & 5 & 0.612026 & 0.122405 & 0.5465 \\
Error & 84 & 18.815686 & 0.223996 & Prob $>$ F \\
C Total & 89 & 19.427713 & & 0.7406 \\
\hline
\end{tabular}

Means for Oneway Anova

Level Number Mean Std Error

$\begin{array}{llll}\text { Blck Creek } & 14 & 0.309357 & 0.12649\end{array}$

$\begin{array}{llll}\text { Congaree } & 15 & 0.527467 & 0.12220\end{array}$

$\begin{array}{llll}\text { Dry Branch } & 15 & 0.312667 & 0.12220\end{array}$

McBean

$\begin{array}{lrrr}\text { Middendorf } & 24 & 0.418792 & 0.09661\end{array}$

$\begin{array}{llll}\text { Pee Dee } & 13 & 0.372385 & 0.13126\end{array}$

$\begin{array}{llll}\text { Tobacco Rd } \quad 9 & 0.268889 & 0.15776\end{array}$

Sid Error uses a pooled estimate of error variance

\section{Means Comparisons}

Dif=Mean[i]-Mean[j] Congaree

Congaree

Pee Dee

Blck Creek

McBean

Middendorf

Dry Branch

Tobacco Rd

Alpha $=0.05$

Comparisons for each pair using Student's $t$
Pee Dee Blck Creek

0.155082

$-0.15508$

$-0.21811$

$-0.10868$

$-0.2148$

$-0.25858$

0.000000

$-0.06303$

0.218110

0.063027

0.000000

0.046407

$-0.05972$

0.109435

0.003310

$-0.04047$
McBean Middendorf Dry Branch

0.214800

0.059718

$-0.00331$

$-0.04641$

$-0.10943$

0.000000

$-0.10612$

$-0.1499$

0.106125

0.000000

$-0.04378$

\section{Tobacco Rd} 0.258578 0.103496 0.040468

0.149903 0.043778 0.000000

1.98862

$$
\begin{array}{|l}
\mathrm{MI} \\
\mathrm{MI} \\
\mathrm{Pr} \\
\mathrm{PO} \\
\mathrm{Po}
\end{array}
$$

\section{$\|$$$
\|
$$

$\mid \begin{aligned} & \mathrm{A} \\ & \mathrm{C} \\ & \mathrm{P} \\ & \mathrm{B} \\ & \mathrm{M} \\ & \mathrm{M} \\ & \mathrm{D} \\ & \mathrm{T} \\ & \mathrm{P}\end{aligned}$$$
\begin{aligned}
& \text { Abs(Dif)- } \\
& \text { Congaree } \\
& \text { Pee Dee } \\
& \text { BIck Creek } \\
& \text { McBean } \\
& \text { Mlddendor } \\
& \text { Dry Branc }
\end{aligned}
$$

Abs(Dif)-LSD
Congaree
Pee Dee
Blck Creek
McBean
Middendorf
Dry Branch
Tobacco Rd

$\begin{array}{rrr}\text { Congaree } & \text { Pee Dee } & \text { Blck Creek } \\ -0.34367 & -0.20156 & -0.13164 \\ -0.20156 & -0.36916 & -0.29948 \\ -0.13164 & -0.29948 & -0.35573 \\ - & & \\ -0.2011 & -0.27771 & -0.20708 \\ -0.12887 & -0.29692 & -0.34644 \\ -0.13826 & -0.30463 & -0.36165\end{array}$

$$
\begin{array}{rrrr}
\text { - } & \text { Middendorf } & \text { Dry Branch } & \text { Tobacco Rd } \\
\text { - } & -0.2011 & -0.12887 & -0.13826 \\
\text { - } & -0.27771 & -0.29692 & -0.30463 \\
\text { - } & -0.20708 & -0.34644 & -0.36165 \\
\text { - } & -0.27169 & -0.20365 & -0.21797 \\
\text { - } & -0.20365 & -0.34367 & -0.35306 \\
& -0.21797 & -0.35306 & -0.44368
\end{array}
$$


FIGURE . $9 \mathrm{~h}$.

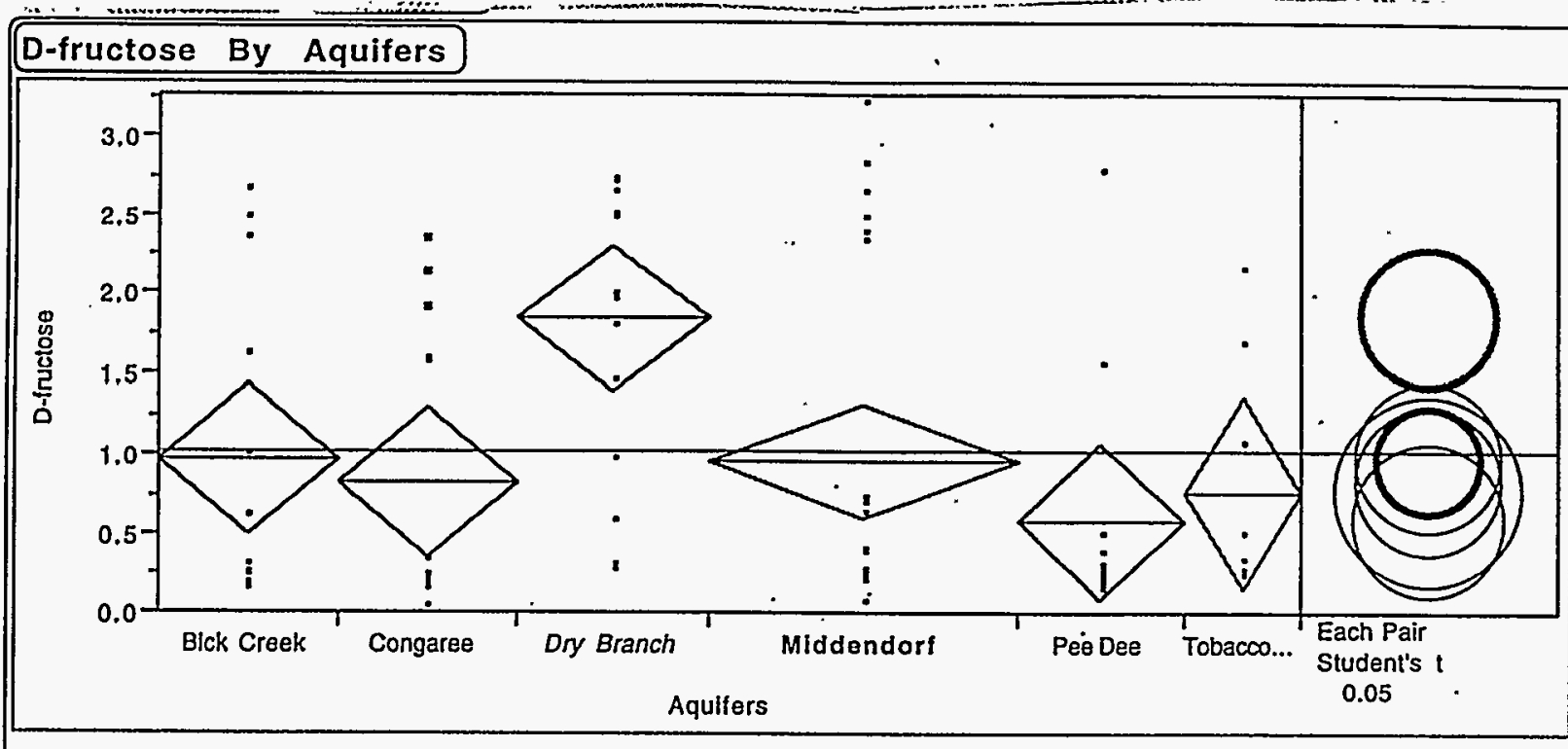

Oneway Anova

Summary of Fit

RSquare

RSquare Ad]

0.172523

Root Mean Square Error

Mean of Response

Observations (or Sum Wgts)

Analysis of Variance

\begin{tabular}{lrrrrr} 
Source & DF & Sum of Squares & Mean Square & F Ratio \\
Model & 5 & & 14.277142 & 2.85543 & 3.4610 \\
Error & 83 & & 68.477971 & 0.82504 & Prob>F \\
C Total & 88 & & 82.755114 & & 0.0069 \\
\hline
\end{tabular}

Means for Oneway Anova

Levol Number

Mean Std Error

Blck Creek

0.96250

Congaree

Dry. Branch

McBean

MIddendorl

Pee Deo

Tobacco Rd

$14 \quad 0.82200$

0.24276

0.24276

.85187

0.23453

Std Error uses a pooled estimate of error variance

\section{Means Comparisons}

Dry Branch

Blck Creok

Middendorf

0.00000

Blck

McBoan

Congareo

Tobacco Rd

$-0.88937$

$-0.90453$ 0.88937
0.00000
0.90453
$-0.01517$
0.01517

Middendor

$-1.02987$

$-0.14050$

$-1.08864$

$-0.19928$

$-0.12533$

Poo Dee

$-1.27841$

$-0.38904$

$-0.18411$

$-0.37387$

McBéa

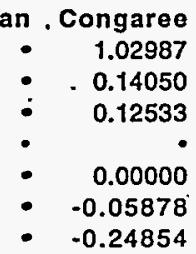

Tobacco Rd 1.08864 0.19928

0.18411

0.05878

0.00000

$-0.18976$
Pee Dee 1.27841 0.38904 0.37387

0.24854 0.18976 0.00000

Alpha $=0.05$

Comparisons for each pair using Student's $t$.

1.98897

Abs(DIf)-LSD

Dry Branch

Blck Creok

Middondorf

McBean

Congaree

Tobacco Rd

Pee Dee

Dry Branch

$-0.65968$

0.218010

0.309906

0.358510

0.326911

0.593823

Blck Creek

0.218010

$-0.68283$

$-0.59239$

Middendorf

McBean Congaree

- 0.358510

-0.59239 \% : -0.54233

$-0.52152$

$-0.54233$

$-0.57259$

$-0.3068$

$-0.48222$

$-0.52203$

$-0.24827$
$-0.48222$

- $\quad-0.68283$

-0.71309
$-\quad-0.4473$
Tobacco Rd

$-0.57259-0.3068$

$-0.52203-0.24827$

$-0.71309 \quad-0.4473$

$-0.85164-0.59364$

$-0.59364-0.70861$ 
FIGURE $9 j$.

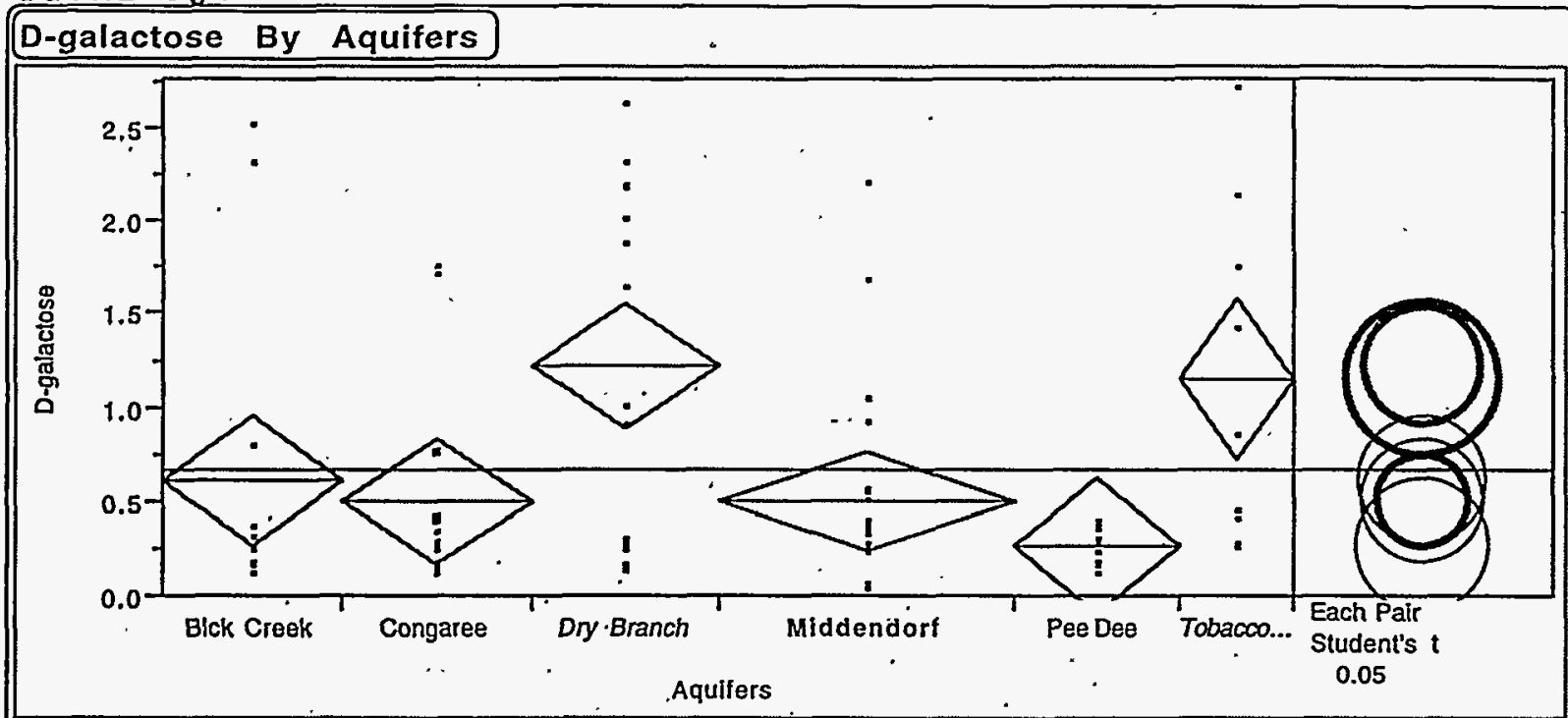

Oneway Anova

\section{Summary of .Fit}

RSquare :-

RSquare Ad] *

Root Mean Square Error

Mean of Response

Observations (or Sum Wgts)

$\ddots$
0.211686
0.164762
0.65998
0.666689
90

\begin{tabular}{|lrrrrr|}
\hline Analysis & of & Variance & & \\
Source & DF & Sum of Squares & Mean Square & F Ratio \\
Model & 5 & 9.825018 & 1.96500 & 4.5113 \\
Error & 84 & 36.588214 & 0.43557 & Prob>F \\
C Total & 89 & 46.413231 & & 0.0011 \\
\hline
\end{tabular}

Means for Oneway Anova

Lovel Number Mean Std Error

$\begin{array}{llll}\text { Blck Creek } & 14 & 0.60864 & 0.17639\end{array}$

$\begin{array}{llll}\text { Congaree } & 15 & 0.50187 & 0.17041\end{array}$

$\begin{array}{llll}\text { Dry Branch } & 15 & 1.21480 & 0.17041\end{array}$

$\begin{array}{lrrrr}\text { McBean } & & 0 & & 0 \\ \text { Mlddendorf } & 24 & 0.50004^{\circ} & 0.13472\end{array}$

$\begin{array}{llll}\text { Pee Des } & 13 & 0.26238 & 0.18305\end{array}$

$\begin{array}{lrrr}\text { Tobacco Rd } & 9 & 1.14656 & 0.21999\end{array}$

Sid Error uses a pooled estimate of error variance

\section{Means Comparisons}

\begin{tabular}{|c|c|c|c|c|c|c|c|}
\hline $\begin{array}{l}\text { Dlf=Mean[I]-Mean[I] } \\
\text { Dry Branch }\end{array}$ & $\begin{array}{r}\text { Dry Branch } \\
0.000000\end{array}$ & $\begin{array}{r}\text { Blck Creek } \\
0.606157\end{array}$ & $\begin{array}{r}\text { Congaree } \\
0.712933\end{array}$ & McBean & $\begin{array}{r}\text { Tobacco Rd } \\
0.068244\end{array}$ & $\begin{array}{r}\text { Middendorf } \\
0.714758\end{array}$ & $\begin{array}{l}\text { Pee Dee } \\
0.952415\end{array}$ \\
\hline Blck Creek & -0.60616 & 0.000000 & 0.106776 & - & -0.53791 & 0.108601 & 0.346258 \\
\hline Congaree & -0.71293 & -0.10678 & 0.000000 & - & -0.64469 & 0.001825 & 0.239482 \\
\hline McBean & - & - & - & - & $\bullet$ & . & - \\
\hline Tobacco Rd & -0.06824 & 0.537913 & 0.644689 & - & 0.000000 & 0.646514 & 0.884171 \\
\hline Middendorf & -0.71476 & -0.1086 & -0.00183 & - & -0.64651 & 0.000000 & 0.237657 \\
\hline Peé Deo & -0.95242 & -0.34626 & -0.23948 & - & -0.88417 & -0.23766 & 0.000000 \\
\hline
\end{tabular}

Alpha $=0.05$

Comparisons for each pair using Student's t

1.98862

Abs(DIf)-LSD

Dry Branch

Blck Creek

Congaree

McBoan

Tobacco Rd

Middendorf

Peo Dee

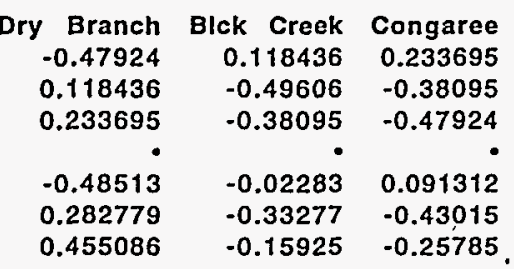

McBean Tobacco Rd Middendorf $-0.48513$

$-0.02283$

0.091312

$-0.61869$

0.133520

0.315055 .* $\cdot 0.282779$

$-0.33277$

$-0.43015$

0.133520

$-0.37887$

$-0.21431$
Pee Dee

0.455086

$-0.15925$

$-0.25785$

0.315055

$-0.21431$

$-0.51478$

Positive values show palrs of means that are significantly different. 


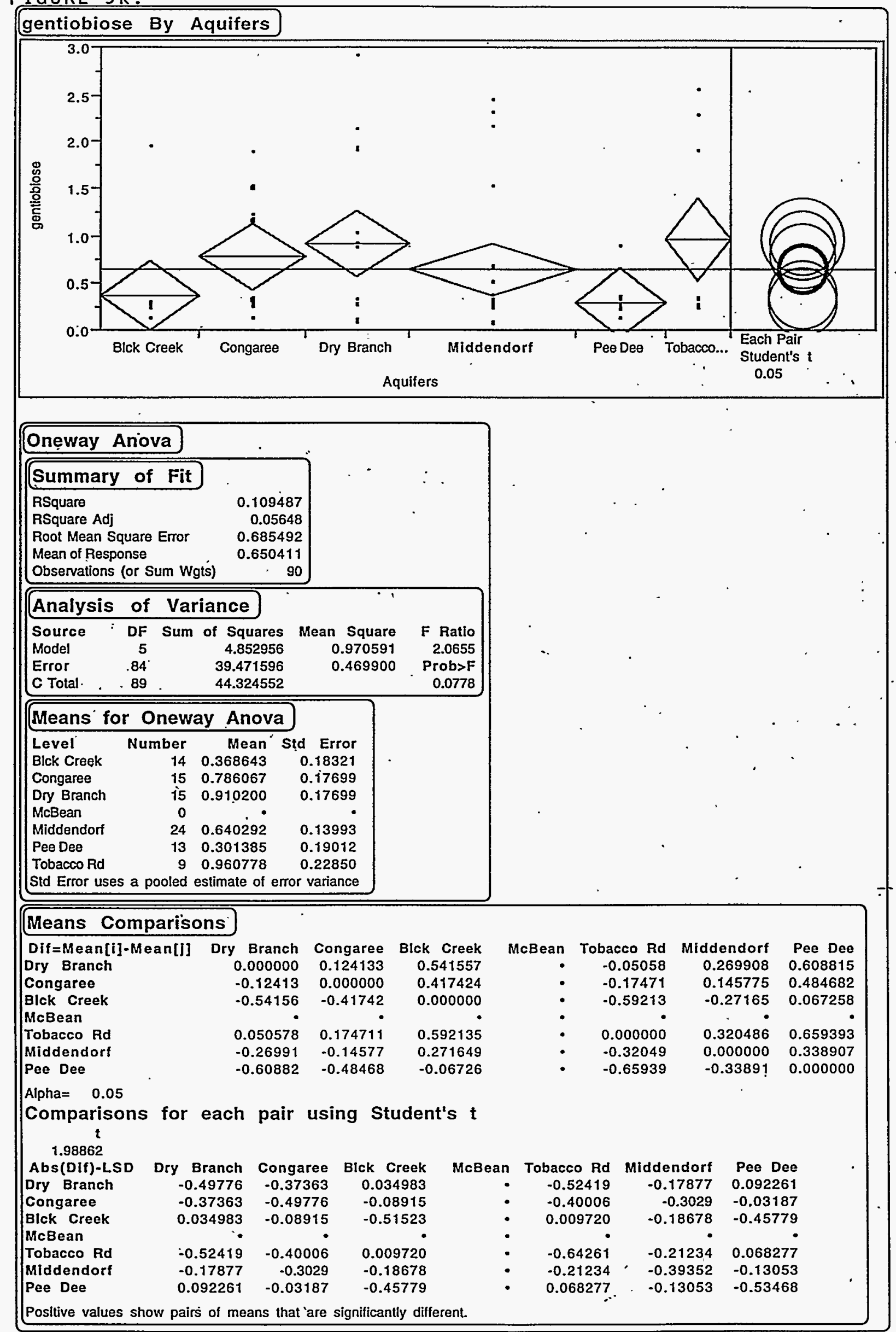


FIGURE 97.

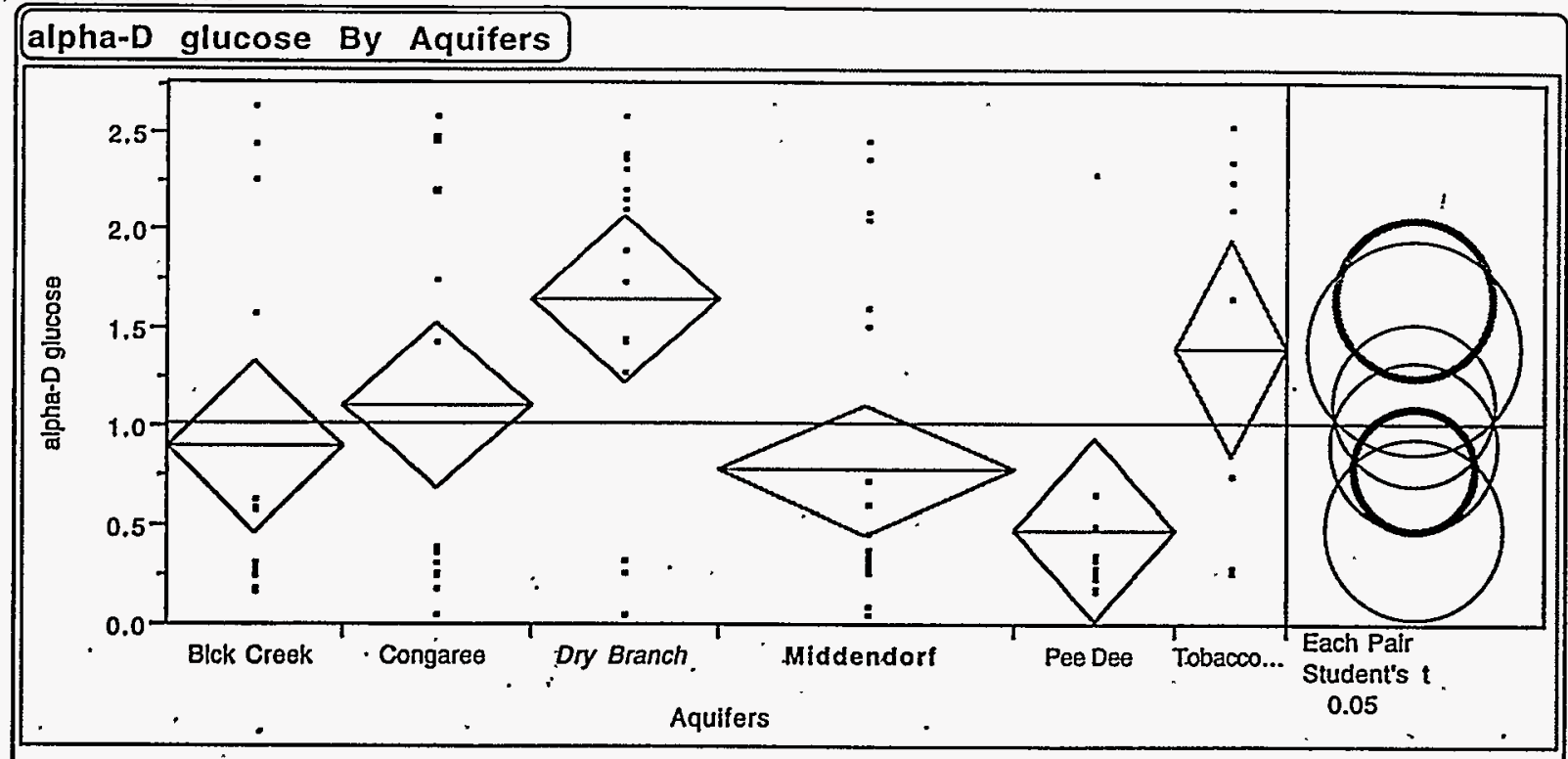

\section{Oneway Anova}

Summary of $\cdot$.Fit

RSquare

RSquare Adj

Root Meán Square Error

Mean of Response

Observations (or Sum Wgts)

\subsection{1 \\ 0.835943 \\ 1.010389}

0.176499

\begin{tabular}{|lrrrrr}
\hline Analysis & of & Variance & & $\ddots$ & \\
Source & DF & Sum of Squares & Mean Square & F Ratio \\
Módel & 5 & 12.580893 & 2.51618 & 3.6007 \\
Error & 84 & 58.699276 & 0.69880 & Prob $>$ F \\
C Total & 89 & & 71.280169 & & .0 .0053 \\
\hline
\end{tabular}

Means for Oneway Anova

Level Number. Mean Std Error

$\begin{array}{llll}\text { Bick Creek } & 14 & 0.89307 & 0.22342\end{array}$

$\begin{array}{llll}\text { Congaree } & 15 & 1.10240 & 0.21584\end{array}$

$\begin{array}{llll}\text { Dry Branch } & 15 & 1.63913 & 0.21584\end{array}$

McBean

Middendort

0.77604

\begin{tabular}{lll} 
Pee Des & 13 & 0.47538 \\
\hline & 9 & 1.38933
\end{tabular}

Tobacco Hd a 91.38933

Std Error uses a pooled estimate of error variance

\section{Means Comparisons}

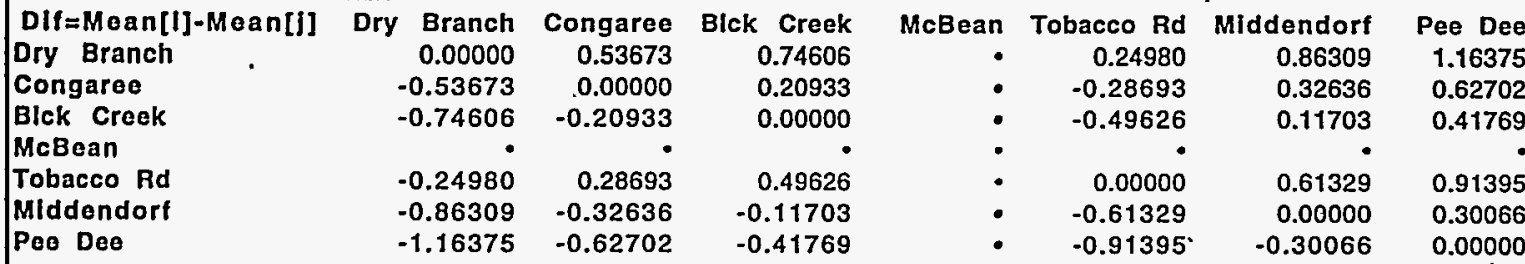

Alpha $=0.05$

Comparisons for each pair using Student's $t$ $t$ 1.98862

\section{Abs(DIf)-LSD}

Dry Branch

Congaree

Blck Creek

McBean

Tobacco Rd

Middendorf

Peo Dee

Dry Branch

$-0.60701$

$-0.07028$

0.128305

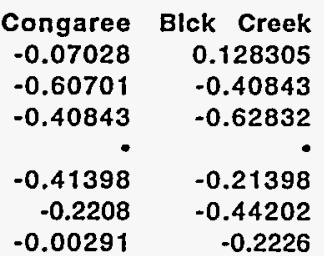
Congaree Blck Creek 0.07028
$-0.00291$

$-0.45112$

0.315938

0.533822
McBean Tobacco Rd Middendorf

$-0.45112$

$-0.41398$

$-0.21398$

$-0.78365$

$-0.03648$

0.193096
0.533822

$-0.2208-0.00291$

$-0.44202-0.2226$

$-0.03648 \quad 0.193096$

$\begin{array}{ll}-0.47989 & -0.27181\end{array}$

$-0.27181-0.65204$

Positive values show pairs of means that are significantly different. 
FIGURE $9 \mathrm{~m}$.

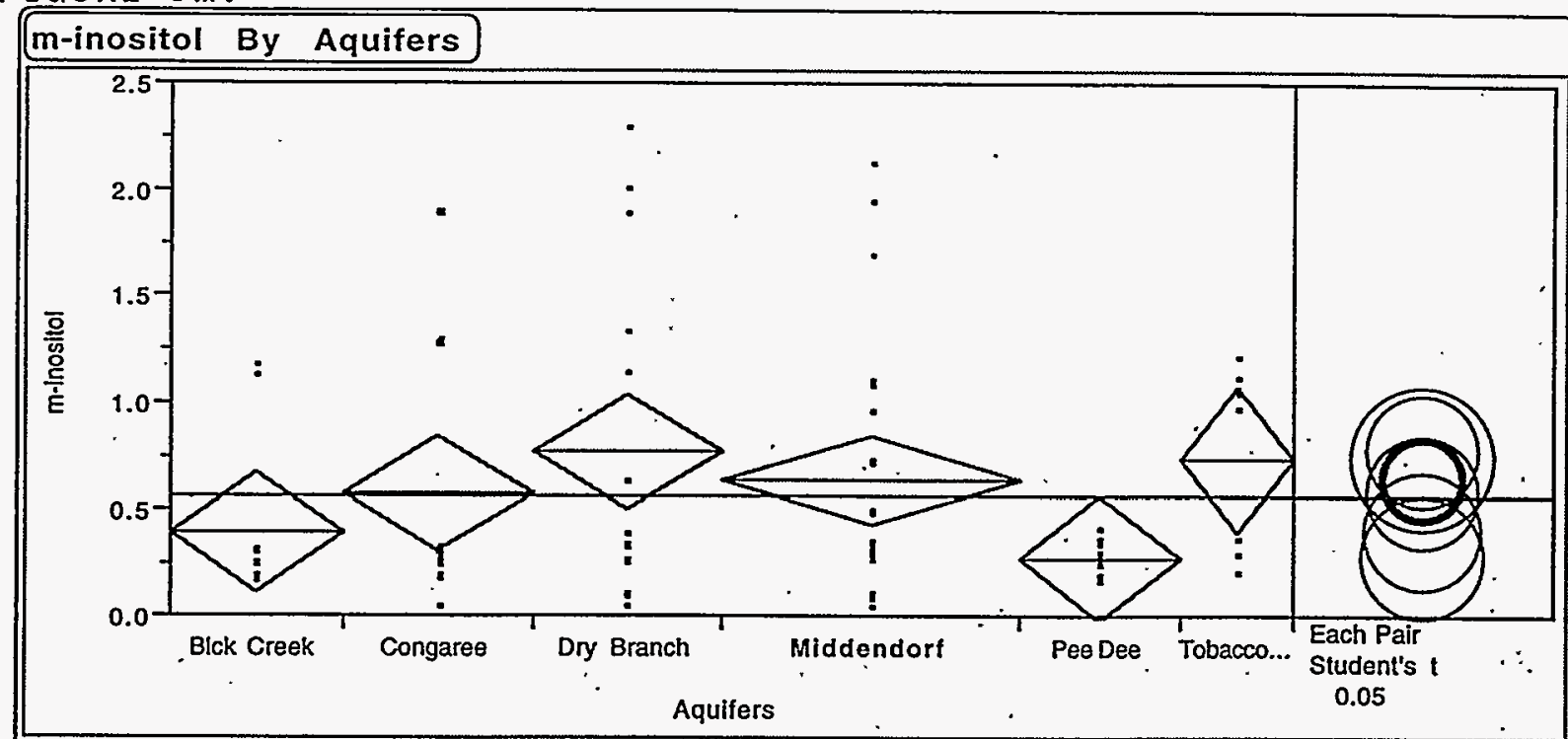

\section{Oneway Anova \\ Summary of Fit}

RSquare

RSquare Adj

Root Mean Square Error

Mean of Response

Observations (or Sum Wgts)

$\begin{array}{r}0.096063 \\ 0.042257 \\ 0.526758 \\ 0.564244 \\ +\quad 90 \\ \hline\end{array}$

Analysis of Variance.

Source - DF Sum of Squares Mean Square F Ratio

Model 5 2476973

Error. $\quad 84 \quad 23.307856$

$\begin{array}{lll}C \text { Total } & 89 & 25.784829\end{array}$

0.495395

$0.277474 \quad$ Prob $>F$

Means for Oneway Ánova

Level Number Mean Std Error

$\begin{array}{llll}\text { Blck Creek } & 14 & 0.391429 & 0.14078\end{array}$

$\begin{array}{llll}\text { Congaree } & 15 & 0.568800 & 0.13601\end{array}$

Dry Branch

McBean

Middendorf

$15 \quad 0.769400$

0

Pee Dee

240.628208

0.13601

Tobacco Rd

130.275692

Std Error uses a pooled estimate of error variance

\section{Means Comparisons}

Dif=Mean[i]-Mean[j] Dry Branch Middendorf Blck Creek Dry Branch

Branch

Middendorf

0.000000

0.141192

0.377971

McBean Tobacco Rd Congaree

- 0.039622

0.200600

$\begin{array}{lll}0.236780 & \bullet & -0.10157 \\ 0.000000 & \bullet & -0.33835\end{array}$

0.059408

$-0.23678$

$-0.37797$

McBean

Tंobacco Rd

$-0.03962$

0.101569

0.338349

$-0.2006$

$-0.05941$

0.177371

Pee Dee

$-0.49371$

$-0: 35252$

$-0.11574$

0.000000

$-0.16098$

0.100978

0.160978

0.000000

$-0.29311$

Pee Dee

0.493708

0.352516

0.115736

Alpha $=0.05$

- -0.45409

0.454085

0.293108

0.000000

Comparisons for each pair using Student's t

1.98862

Abs(Dif)-LS

Dry Branch

Middendorf

Blck Creek

McBean

Tobacco Rd

Congaree

Pee Dee
Dry Branch

$-0.3825$

$-0.20359$

$-0.0113$

$-0.40205$

$-0.1819$

0.096768
Middendorf Blck Creek
$-0.20359$
$-0.30239$
$-0.1155$
$-0.0113$
$-0.1155$
$-0.39593$
$-0.30787$
$-0.28537$
$-0.00822$
$-0.1092$
$-0.2119$
$-0.28773$

McBean Tobacco Rd

Congaree

$-0.1819$

$-0.28537$

$-0.2119$

$-0.30787$

.0 .1092

.

$-0.2807$

$-0.3825$

$-0.2807$

$-0.10383$

Pee Dee

0.096768

$-0.00822$

$-0.28773$

$-0.00015$

$-0.10383$

$-0.41087$ 
EIGURE $9 n$

alpha-D lactose By Aquifers

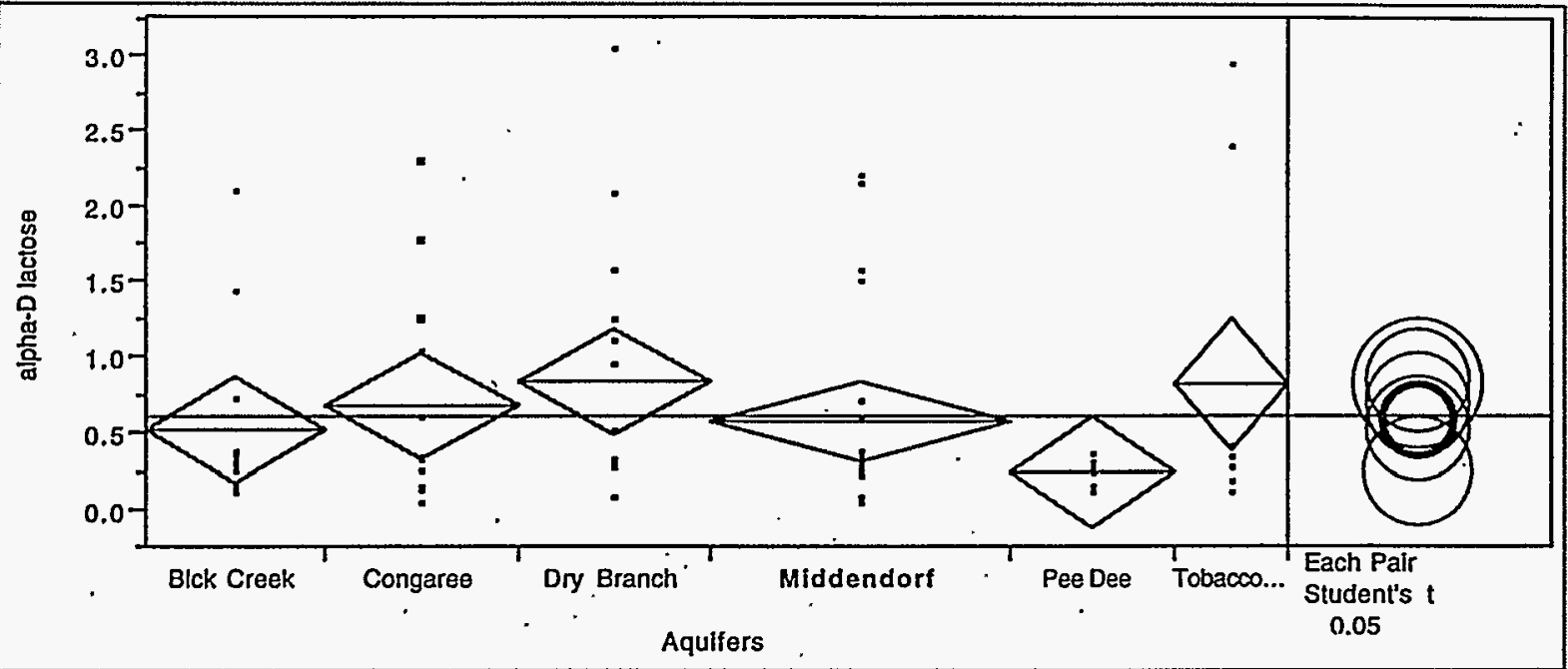

\section{Oneway Anova}

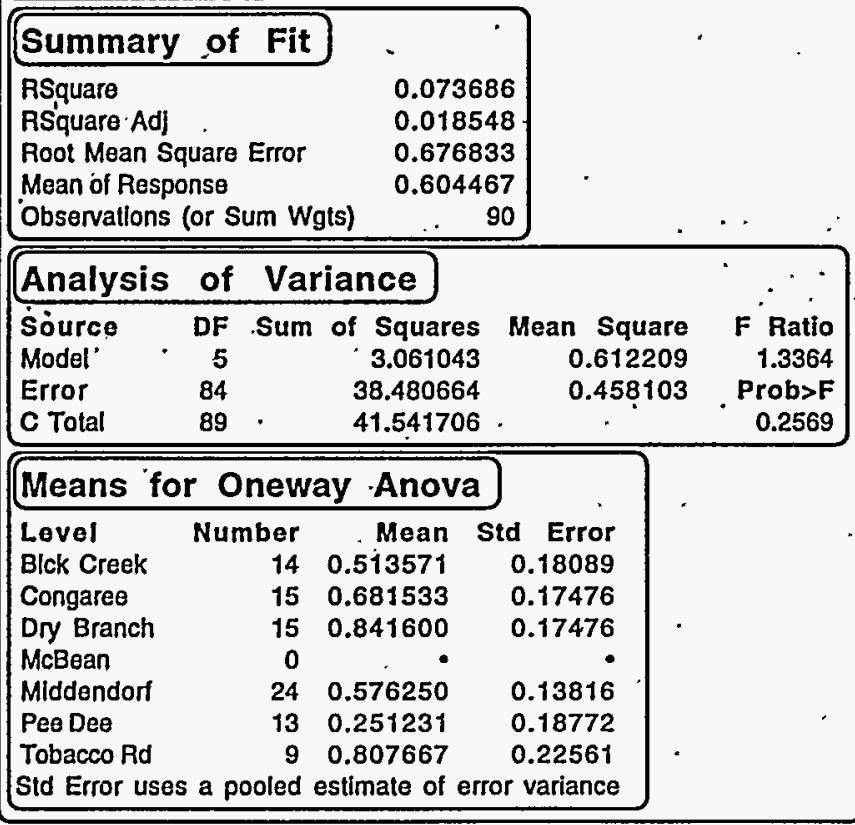

\section{Means Comparisons}

Dif=Mean[1]-Mean[1]'. Dry Branch

Dry Branch

Congaree

Blck Creek

McBean

Tobacco Rd

Middendorf

Pee Dee

Alpha $=\cdot 0.05$

Comparisons for each pair using Student's t
-

$0.000000 \quad 0.160067$

$-0.16007$

$-0.32803$

0.000000

$-0.16796$

$-0.03393$

$-0.26535$

$-0.59037$

0.126133

$-0.10528$

$-0.4303$
Congaree Blck Creek
0.328029

0.167962

0.000000

0.294095

0.062679

$-0.26234$
McBean Tobacco Rd Middendorf 0.265350

0.105283

$-0.06268$

0.231417

0.000000

$-0.32502$
Pee Dee 0.590369 0.430303 0.262341 0.556436 0.325019 0.000000
1.98862

Abs(DIf)-LSD

Dry Branch

Congaree

Blck Creok

McBean

Tobacco Rd

Middendorf

Pee Dee

\section{Dry Branch Congaree Blck Creek $\begin{array}{lll}-0.49148 & -0.33141 & -0.17215\end{array}$ $-0.33141 \quad-0.49148$ $-0.17215 \quad-0.33221$ \\ $\begin{array}{ll}-0.53357 & -0.44137\end{array}$ \\ $\begin{array}{ll}-0.17766 & -0.33773\end{array}$ \\ -0.17215
-0.33221 \\ $-0.50873$ \\ $-0.28096$ \\ $-0.38996$}

$0.080340,-0.07973$
$-0.25608$
McBean Tobacco Rd Middendorf $-0.17766$

$-0.33773$

$-0.38996$

0.090

$-0.29468$

$-0.38855$

$-0.13849$
Pee Dee

0.080340

$-0.07973$

$-0.25608$

$-0.02721$

$-0.13849$

$-0.52793$

Positive values show pairs of means that are significantly different. 


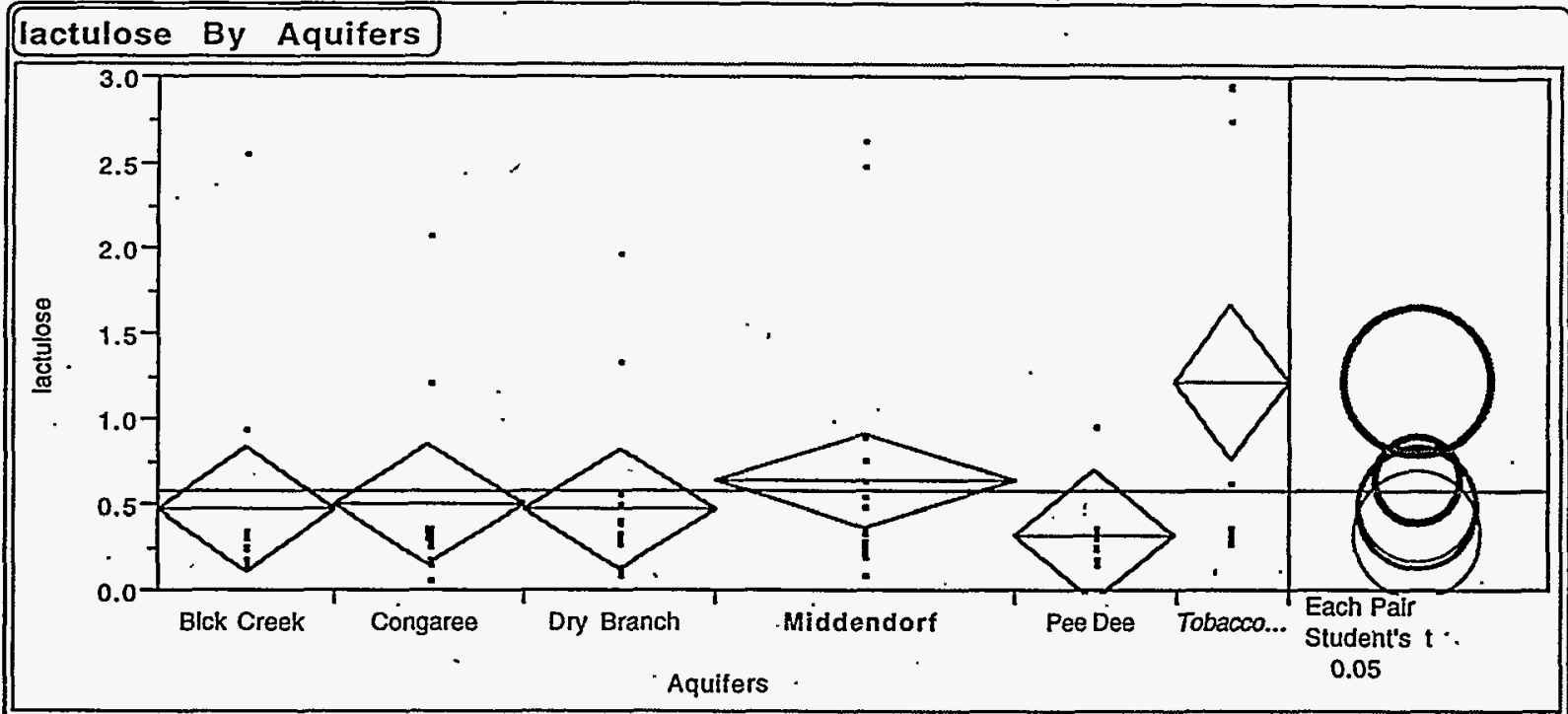

\section{Oneway Anova}

Summary of Fit

RSquare

RSquare Adj

0.112122

Root Mean Square Error

Mean of Response

Observations (or Sum Wgts)

0.059272

0.684463

0.575678 90

Analysis of Variance

Source DF Sum of Squares Mean Square F Ratio

$\begin{array}{lllll}\text { Model } & 5 & 4.969540 & 0.993908 & 2.1215\end{array}$

$\begin{array}{lllll}\text { Error } & 84 & 39.353072 & 0.468489 & \text { Prob }>\text { F }\end{array}$

$\begin{array}{lllll}\text { C Total } & 89 & -44.322612 & 0.0707\end{array}$

Means for Oneway Anova

Level Number Mean Std Error

$\begin{array}{llll}\text { Blck Creek } & 14 & 0.47050 & 0.18293\end{array}$

$\begin{array}{llll}\text { Congaree } & 15 & 0.50907 & 0.17673\end{array}$

$\begin{array}{llll}\text { Dry Branch } & 15 & 0.47940 & 0.17673\end{array}$

Mry Bran

McBean

Pee Dee

Tobacco Rd

Std Error uses a pooled estimate of error variance

\section{Means Comparisons}

Dif=Mean[i]-Mean[i] Middendorf

\section{Middendorf}

Dry Branch

Blck Creek

McBean

Tobacco Rd

Congaree

Pee Dee

Alpha $=0.05$

Comparisons for each pair using Student's t

$\begin{array}{rrr}\text { iddendorf } & \text { Dry Branch } & \text { Blck Creek } \\ 0.000000 & 0.155058 & 0.163958 \\ -0.15506 & 0.000000 & 0.008900 \\ -0.16396 & -0.0089 & 0.000000 \\ \ddots & \bullet & \\ 0.582764 & 0.737822 & 0.746722 \\ -0.12539 & 0.029667 & 0.038567 \\ -0.31023 & -0.15517 & -0.14627\end{array}$

0.000000

$-0.16396$

$-0.12539$

$-0.15517$
1.98862

Abs(Dif)-LSD Middendorf Dry Branch Blck Creek Middendorf

Dry Branch

Blck Creek

McBean

Tobacco Rd

Congaree

Peé Dee

- -0.39293

$-0.29295$

$-0.29379$

0.050740

$-0.32261$

$-0.1585$
McBean Tobacco Rd Congaree

- $\quad 0.050740 \quad-0.32261$

- $\quad 0.163918 \quad-0.46735$

- $\quad 0.165182 \quad-0.46725$

-

$\begin{array}{ll}-0.64164 & 0.134251\end{array}$

$0.134251-0.49702$

0.302764

$-0.33094$
Congaree

0.125392

$-0.02967$

$-0.03857$

0.708156

0.000000

$-0.18484$
Pee Dee 0.310228 0.155169 0.146269

0.892991

0.184836

0.000000

Positive values show pairs of means that are significantly different. 
FIGURE $9 p$.

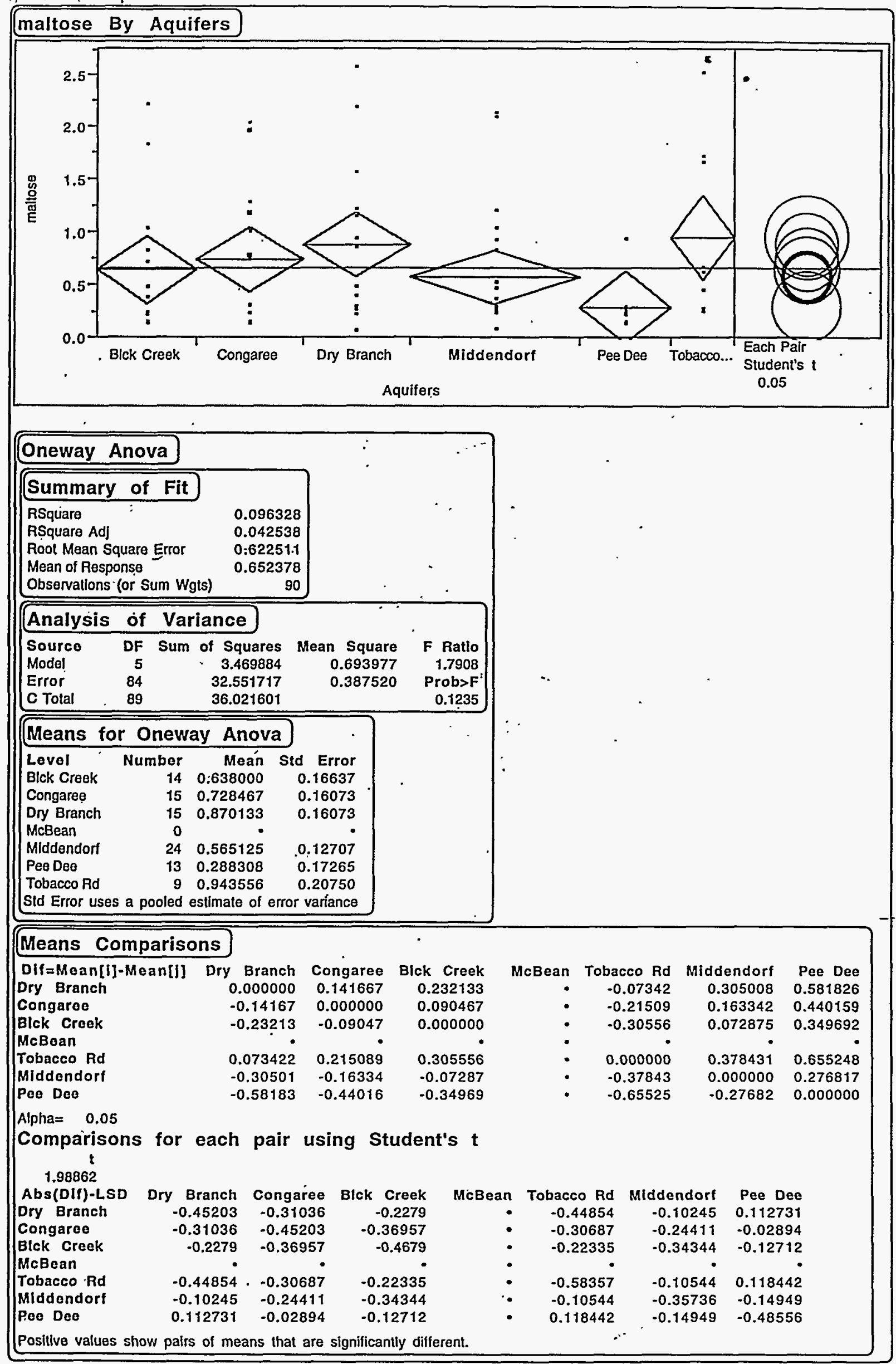


EIGURE 90

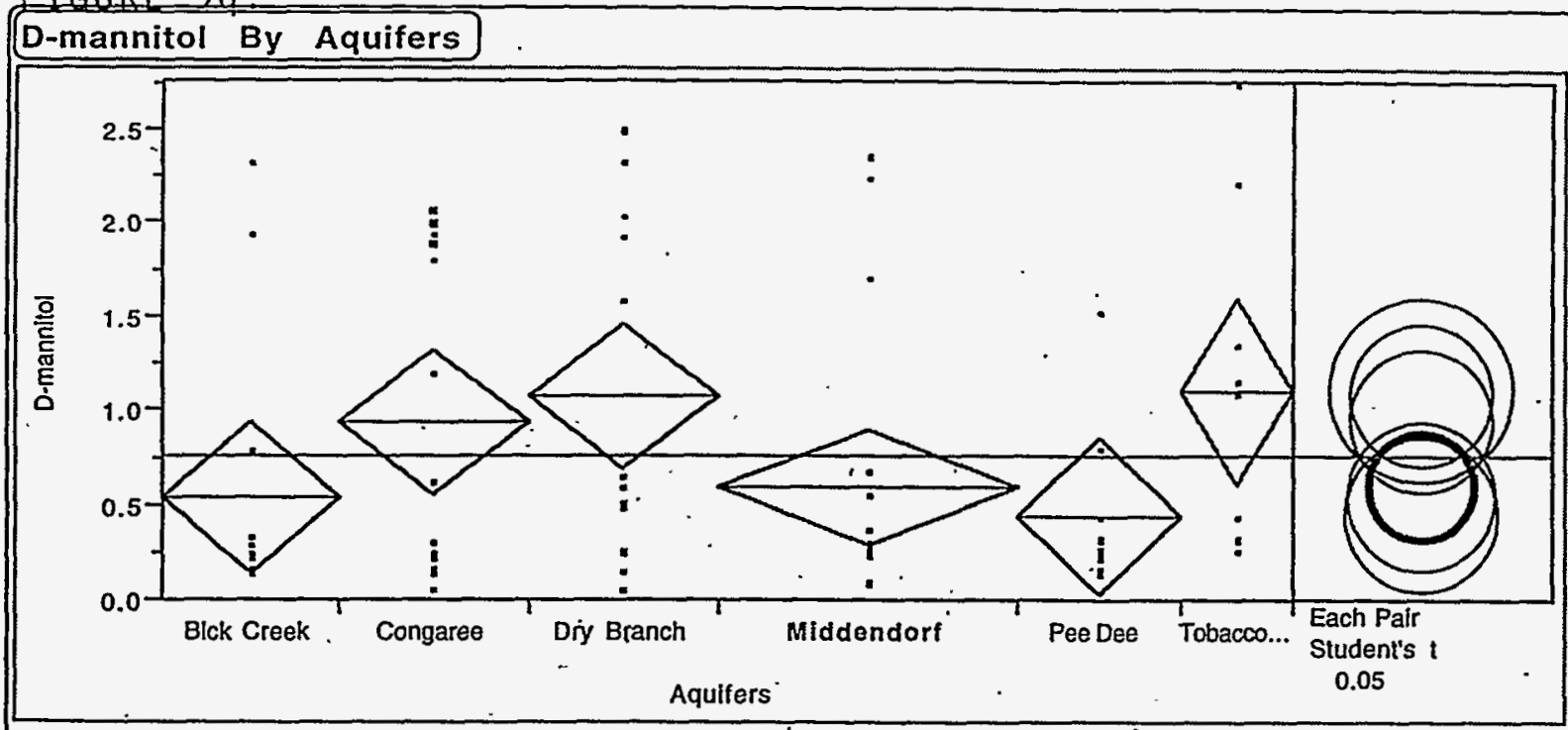

\section{Oneway Anova}

Summary of Fit

RSquare

RSquare Adj

Root Mean Square Error

Mean of Response

Observations (or Sum Wgts)

$\begin{array}{r}0.103303 \\ 0.049928 \\ 0.756553 \\ 0.7556 \\ 90 \\ \hline\end{array}$

\begin{tabular}{|lrrrrr|}
\hline Analysis & of & Variance & & & \\
Source & DF & Sum of Squares & Mean Square & F Ratio \\
Model & 5 & 5.538908 & 1.10778 & 1.9354 \\
Error & 84 & 48.079261 & 0.57237 & Prob>F \\
C Total & 89 & 53.618170 & & 0.0970 \\
\hline
\end{tabular}

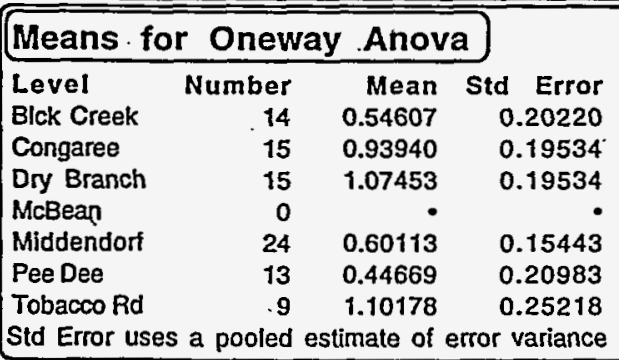

Sta Error uses a pooled estimate of escor variance

\section{Means Comparisons}

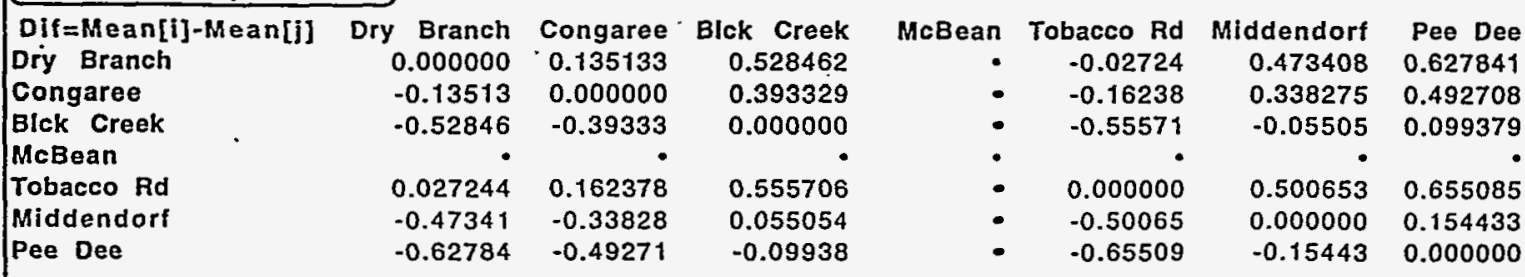

Pee Dee

$-0.62784-0.49271$

$-0.09938$

Alpha $=0.05$

Comparisons for each pair using Student's $t$ $\mathbf{t}$

$$
1.98862
$$

Abs(Dlf)-LSD Dry Branch Congaree Blck Creek

Dry Branch

Congaree

Blck Creek

McBean

Tobacco Rd

$$
-0.54936
$$

$-0.41423$

$-0.03063$

$-0.41423$

$-0.03063$

$-0.54936 \quad-0.16576$

$-0.16576$

$-0.60711$

$-0.02178$

$-0.47197$

$-0.15691$

$\begin{array}{lll}0.057739 & -0.07739 & -0.4801\end{array}$

$-0.56865$

$-0.08708$

$-0.4509$

Pee Dee

McBean Tobacco Rd Middendorf

$\begin{array}{ll}-0.02178 & 0.057739\end{array}$

$-0.15691$

-0.60711
-0.47197

$-0.4509$

$-0.07739$

$-0.08708$

$\bullet$

$\begin{array}{lll}-0.70923 & -0.08741 & 0.002693\end{array}$

-0.70923
-0.08741

0.002693

$-0.43431$

$-0.36367$

$-0.36367$

$-0.59011$

Positive values show pairs of means that are significantly different. 
FIGURE $9 r$.

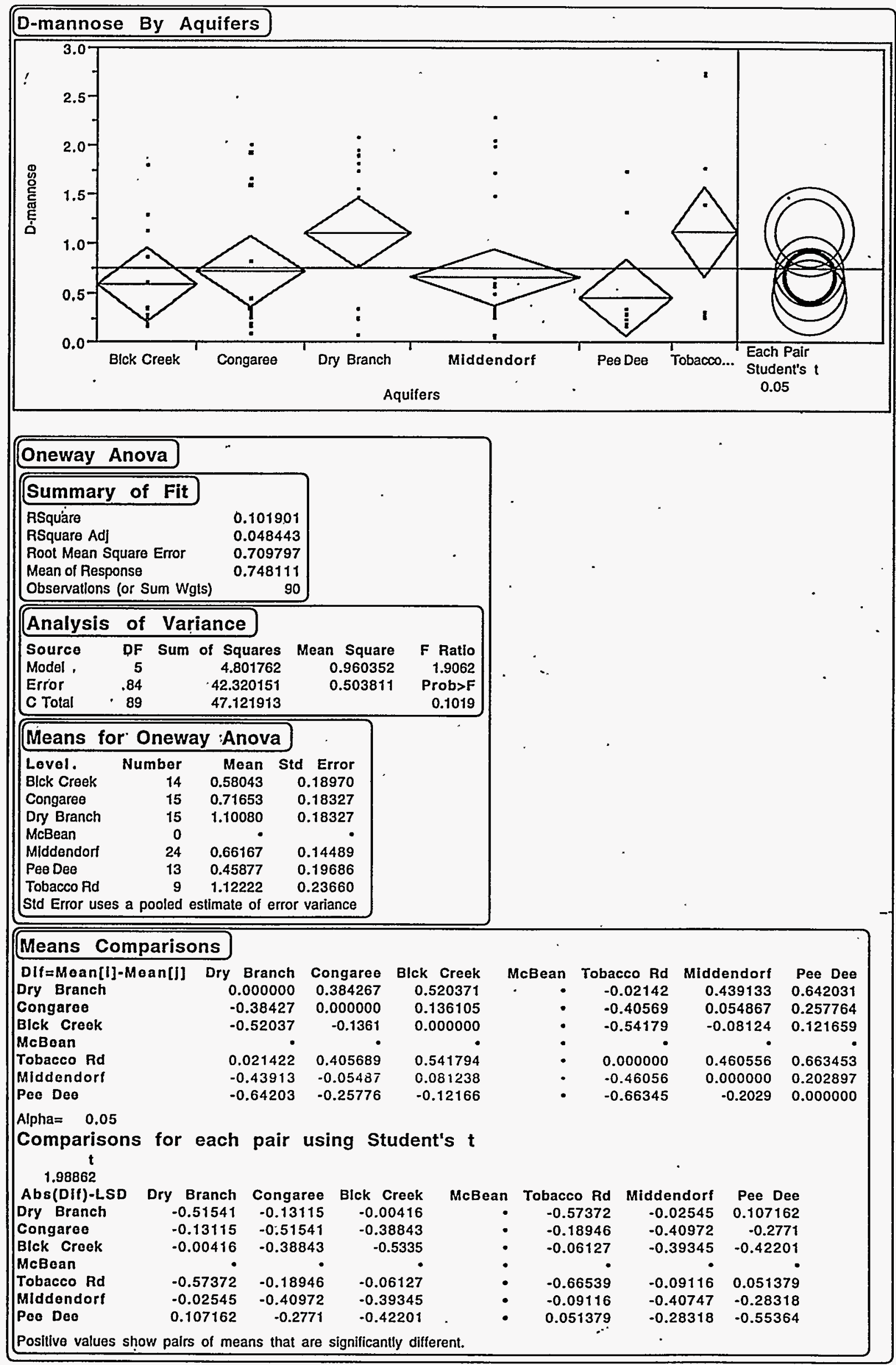


FIGURE $9 \mathrm{~s}$. -

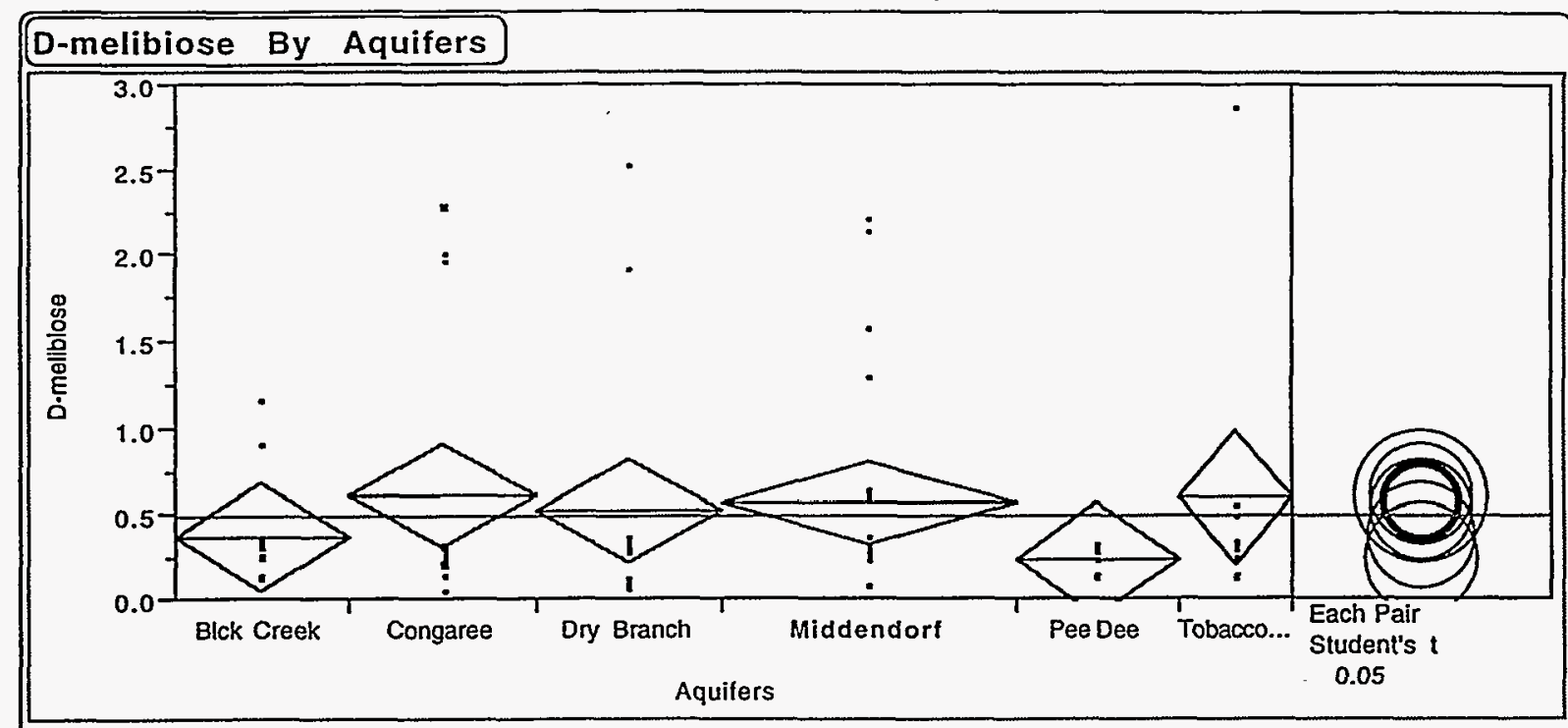

\section{Oneway Anova}

\section{Summary of Fit}

RSquare

RSquare Adj

0.044221

Root Mean Square Error

Mean of Response

$-0.01267$

0.607086

0.488133

Observations (or Sum Wgts) 90

\section{Analysis of Variance}

Source DF Sum of Squares Mean Square F Ratio

$\begin{array}{lllll}\text { Model } & 5 & 1.432355 & 0.286471 & 0.7773\end{array}$

$\begin{array}{lllll}\text { Error } & 84 & 30.958447 & 0.368553 & \text { Prob }>F\end{array}$

\begin{tabular}{llll}
$\mathrm{C}$ Total & 89 & 32.390802 & 0.5688 \\
\hline
\end{tabular}

Means for Oneway Ȧnova

Level Number Mean Std Error

Bick Creek $\quad 14 \quad 0.3709290 .16225$

$\begin{array}{llll}\text { Congaree . } \quad 15 & 0.598067 & 0.15675\end{array}$

$\begin{array}{lrrr}\text { Dry Branch } & 15 & 0.520067 & 0.15675\end{array}$

McBean

Middendorf

Pee Dee

240.562500

0.12392

$13 \quad 0.239000$

0.16838

Std Error uses a pooled estimate of error variance

\section{Means Comparisons}

Dif=Mean[i]-Mean[j] Congaree

\section{Congaree}

Dry Branch

Blck Creek

McBean

Tobacco Rd

Middendort

Pee Dee

Alpha $=0.05$

Comparisons for each pair using Student's t
Dry Branch Blck Creek $0.078000 \quad 0.227138$ $0.000000 \quad 0.149138$ $\begin{array}{ll}-0.14914 & 0.000000\end{array}$

0.0714

$-0.00251$

$-0.03557$

$-0.35907$
0.075489

0.042433

$-0.28107$
0.224627

0.191571

$-0.13193$
McBean Tobacco Rd Middendorf 0.002511

$-0.07549$

$-0.22463$

0.000000

$-0.03306$

$-0.35656$ 0.359067 $\begin{array}{ll}-0.04243 & 0.281067\end{array}$ $\begin{array}{ll}-0.19157 & 0.131929\end{array}$ 0.033056 0.000000 $\begin{array}{ll}-0.3235 & 0.000000\end{array}$
1.98862 Abs(Dif)-LSD Congaree Dry Branch Blck Creek McBean Tobacco Rd Middendorf Pee Dee

Congaree $-0.44083$ $-0.36283$ $-0.22149$ $-0.50651$ $-0.36179$ $-0.0984$
Dry Branch Blck Creek $-0.36283$

$-0.44083$

$-0.29949$ $-0.22149$

$-0.29949$

$-0.4563$

$-0.43354$

$-0.35493$

$-0.1764$
$-0.29117$

$-0.21443$

$-0.33307$
McBean Tobacco Rd Middendort $-0.36179$

$-0.35493$

$-0.21443$

$-0.43354$

$-0.29117$

$-0.56911$

$-0.43882$

$-0.16695$

$-0.43882$

$-0.34851$

$-0.09224$
Pee Dee $-0.0984$

$-0.1764$

$-0.33307$

$-0.16695$

$-0.09224$

$-0.47353$

Positive values show pairs of means that are significantly different. 


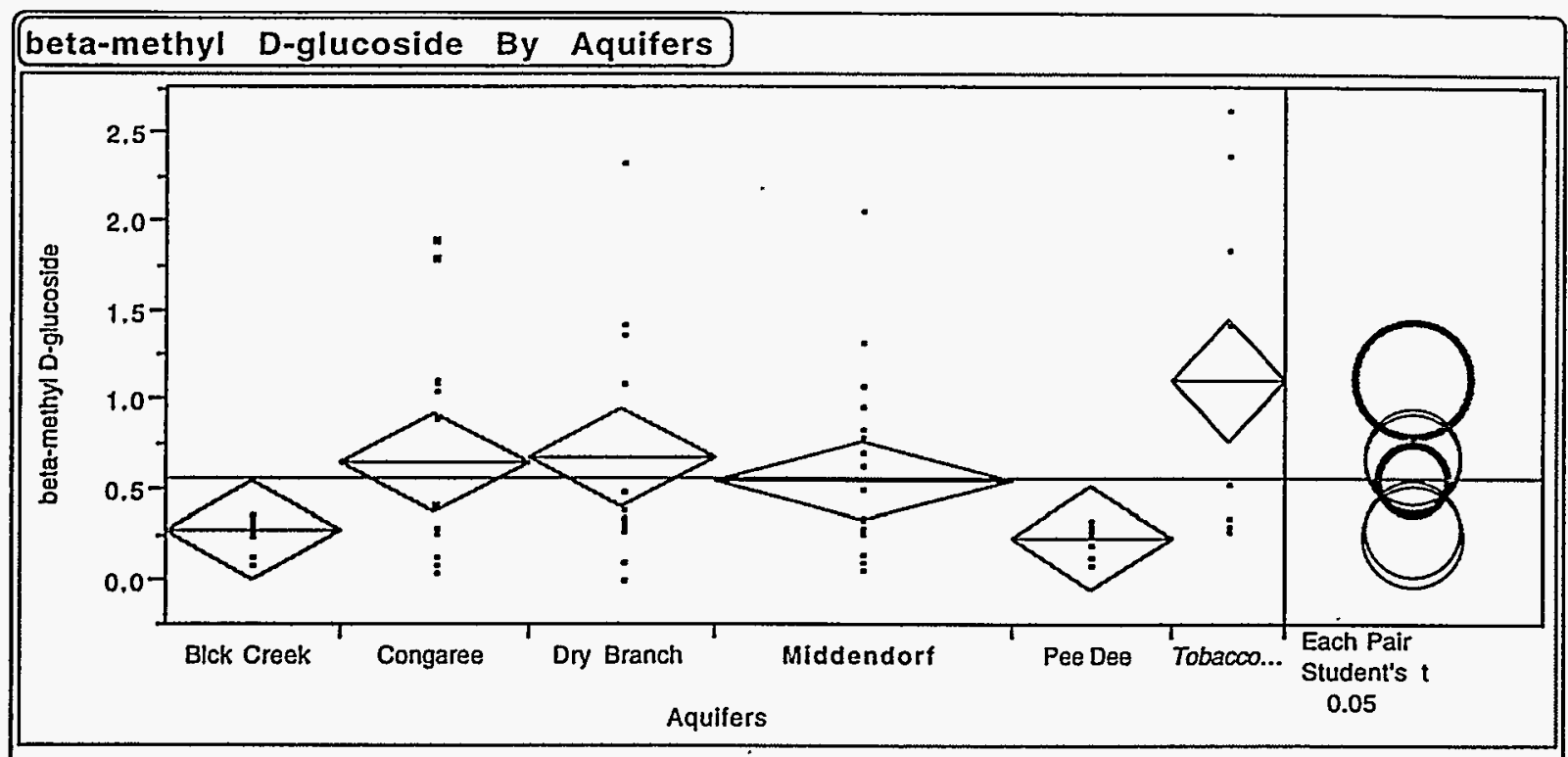

\section{Oneway Anova}

\section{Summary of Fit}

RSquare .

Rsquare Adj

0.191073

Root Mean Square Error

Mean of Response

Observations (or Sum Wgts)

\section{Analysis of Variance}

\begin{tabular}{|lrrrrr} 
Source & DF & Sum of Squares & Mean Square & F Ratio \\
Model & 5 & 5.671908 & 1.13438 & 3.9683 \\
Error & 84 & 24.012595 & 0.28586 & Prob $>$ F \\
C Total & 89 & 29.684503 & & 0.0028 \\
\hline
\end{tabular}

Means for Oneway Anova

Level Number Mean Std Error

$\begin{array}{llll}\text { Blck Creek } & 14 & 0.26800 & 0.14289\end{array}$

$\begin{array}{llll}\text { Congaree } & 15 & 0.65453 & 0.13805\end{array}$

Dry Branch

McBean

Middendori

0.13805

Peo Dee

150.68153

0.13805

Tobacco Rd

$\begin{array}{ll}24 & 0.54654 \\ 13 & 0,23415\end{array}$

0.10914

0.14829

Std Error uses a pooled estimate of error variance

\section{Means Comparisons}

Dif=Mean[I]-Mean[j] Dry Branch

Dry Branch

Congaree

Blck Creek

McBean

Tobacco Rd

Middendorf

Pee Deo

Branch
0.000000

$-0.027$

$-0.41353$

0.430244

$-0.13499$

$-0.44738$

Congaree

0.027000

0.000000

$-0.38653$

0.457244

$-0.10799$

$-0.42038$

Blck Creek

0.413533

0.386533

0.000000

0.843778

0.278542

$-0.03385$
McBean Tobacco Rd Middendorf

- $\quad-0.43024$

- $\quad-0.45724$

- $\quad-0.84378$

- 0.000000

$-0.56524$

- -0.87762
0.134992

0.107992

$-0.27854$

0.565236

0.000000

$-0.31239$
Pee Dee 0.447379 0.420379 0.033846

0.877624 0.312388 0.000000

Alpha $=0.05$

Comparisons for each pair using Student's t t

1.98862 Abs(Dif)-LSD

Dry Branch

Congareo

Blck Creek

McBean

Tobacco Rd

Middendorf

Peo Dee

Posilive val
McBean Tobacco Rd

Tobacco Rd

- $\quad 0.008943$

- 0.389512

. 0.389512

- $\quad 0.50122$

- 0.149650

- $\quad 0.416572$
Middendorf $-0.21496$

$-0.24196$

$-0.07902$

0.149650

$-0.30693$

$-0.05376$
Pee Dee

0.044483

0.017483

$-0.37568$

0.416572

$-0.05376$

$-0.41704$ 
FIGURE $9 u$.

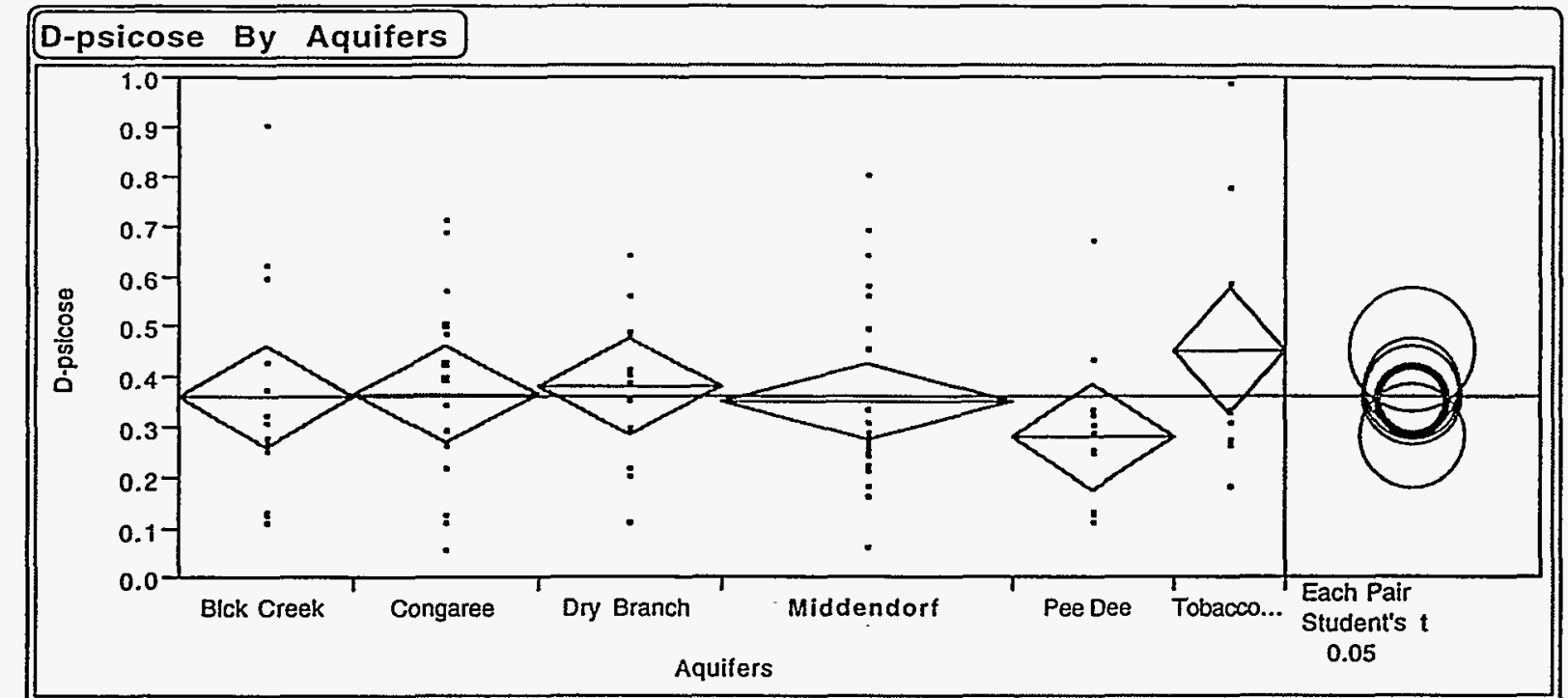

\section{Oneway Anova}

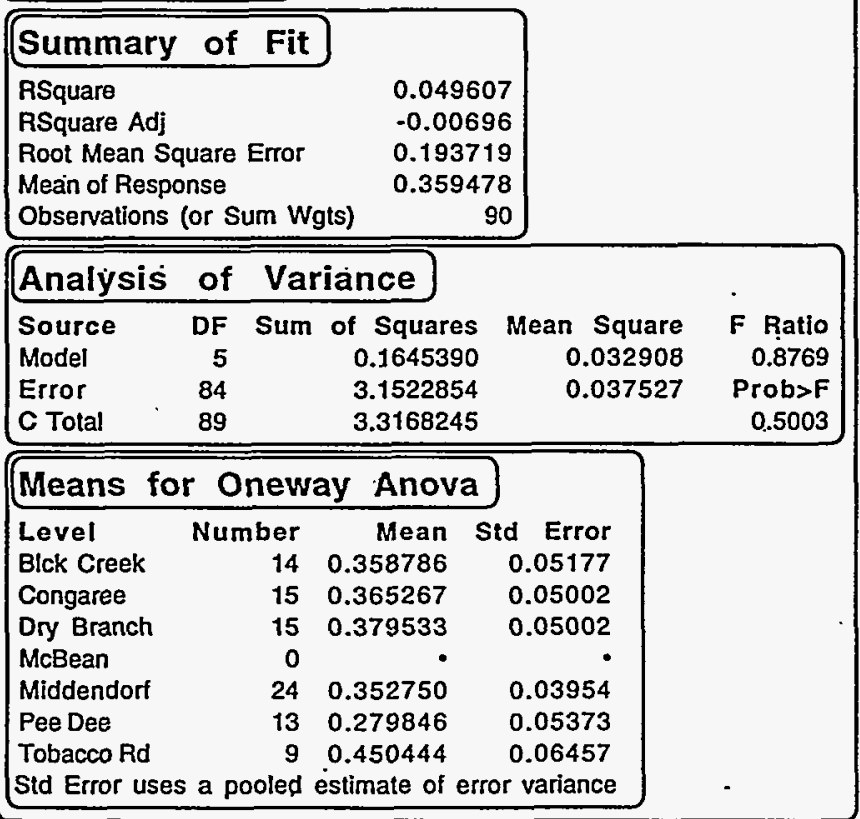

\section{Means Comparisons}

\begin{tabular}{|c|c|c|c|c|c|c|c|}
\hline Dif $=$ Mean [i] -Mean[i] & Dry Branch & Congaree & Blck Creek & McBean & Tobacco Rd & Middendorf & Pee Dee \\
\hline Dry Branch & 0.000000 & 0.014267 & 0.020748 & - & -0.07091 & 0.026783 & 0.099687 \\
\hline ingaree & -0.01427 & 0.000000 & 0.006481 & - & -0.08518 & 0.012517 & 0.085421 \\
\hline ck Creek & -0.02075 & -0.00648 & 0.000000 & - & -0.09166 & 0.006036 & 0.078940 \\
\hline cBean & - & - & 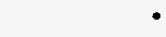 & - & - & - & \\
\hline & 0.070911 & 0.085178 & 0.091659 & - & 0.000000 & 0.097694 & 0.170598 \\
\hline de & -0.02678 & -0.01252 & -0.00604 & - & -0.09769 & 0.000000 & 0.072904 \\
\hline De & -0.09969 & -0.08542 & -0.07894 & 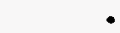 & -0.1706 & -0.0729 & 0.00000 \\
\hline
\end{tabular}

Pee Dee

$\begin{array}{lll}-0.09969 & -0.08542 & -0.07894\end{array}$

Alpha $=\mathbf{0 . 0 5}$

Comparisons for each pair using Student's $t$

$$
1
$$

1.98862

Abs(Dif)-LSD Dry Branch Congaree Blck Creek McBean Tobacco Rd Middendorf Pee Dee

Congaree

$-0.14067$

$-0.1264$

$-0.1264$

$-0.12241$

$-0.14067$

$-0.13668$

$-0.1456$

Blck Cre

$-0.12241$

$-0.13668$

$-0.09152$

$-0.07293$

Tobacco Rd

Middendorf

$-0.04629$

$-0.11428$

$-0.12352$

$-0.06944$

McBean Tobacco Rd Middendort

$-0.11428$

$-0.07293$

$-0.1816$

.0 .05288

0.003550

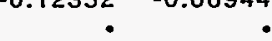

$-0.05288 \quad 0.003550$

$-0.11121-0.05976$

$-0.05976-0.1511$

Positive values show pairs of means that are significantly different. 
'FIGUREE 9v.

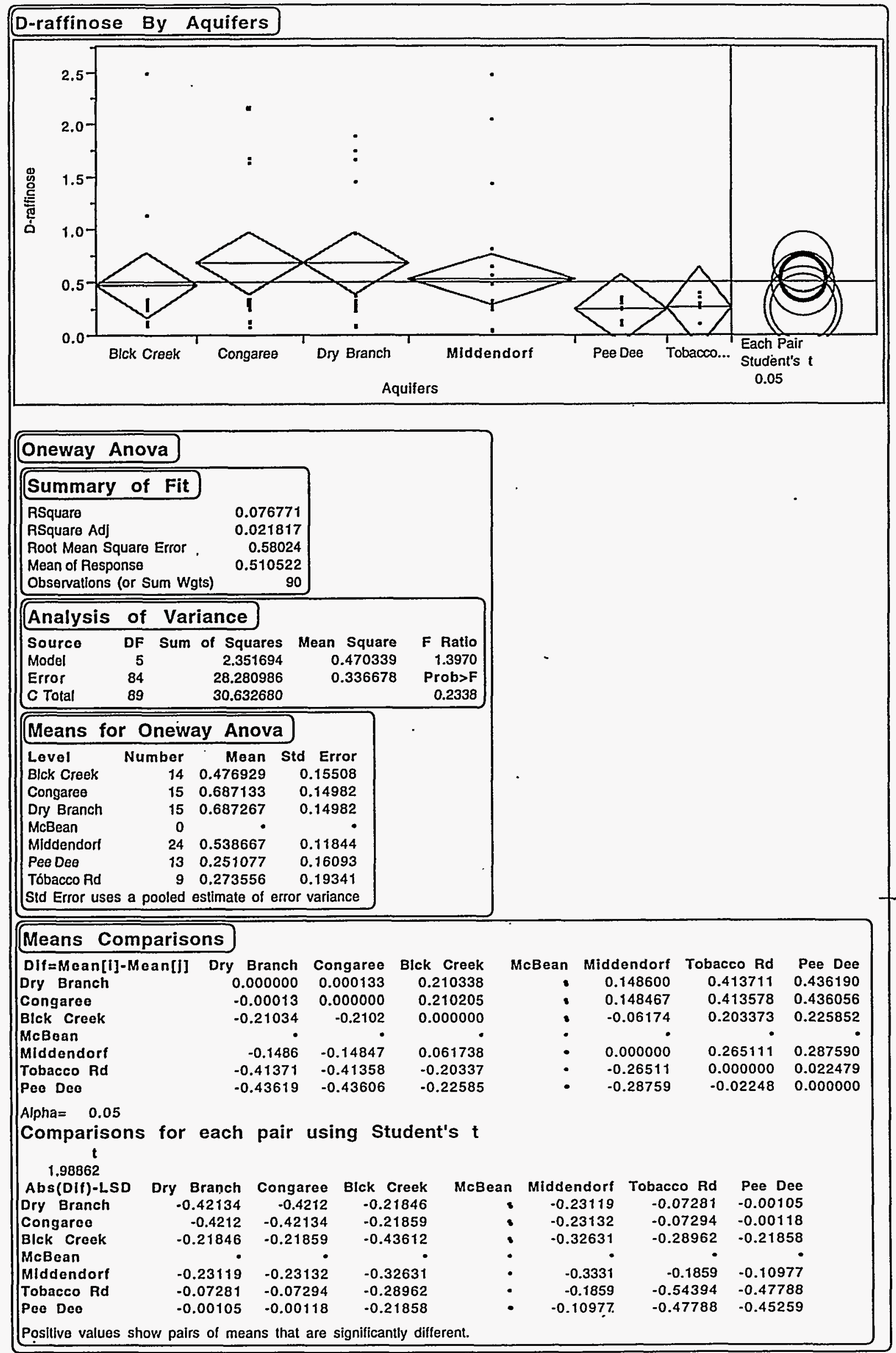


FIGURE $9 \mathrm{~W}$.

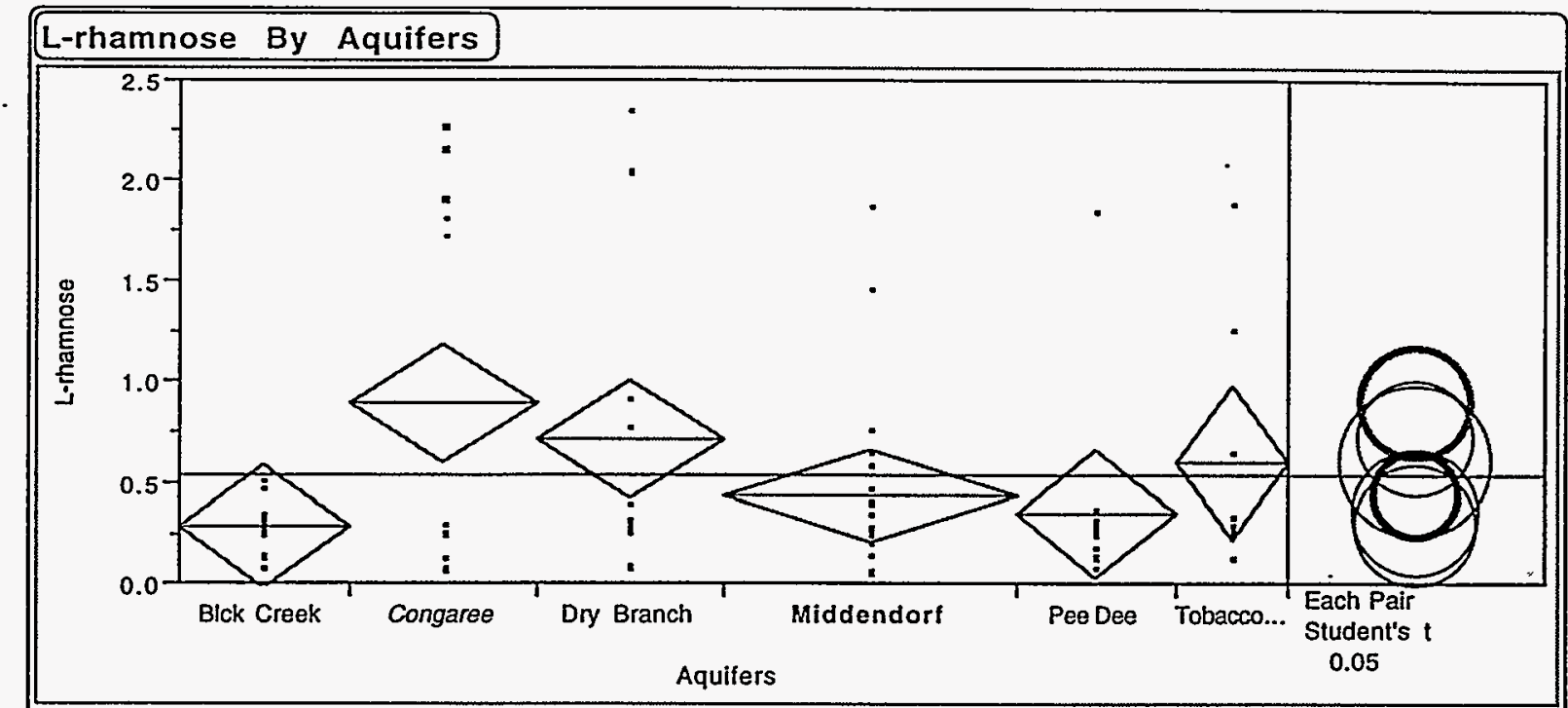

\section{Oneway Anova}

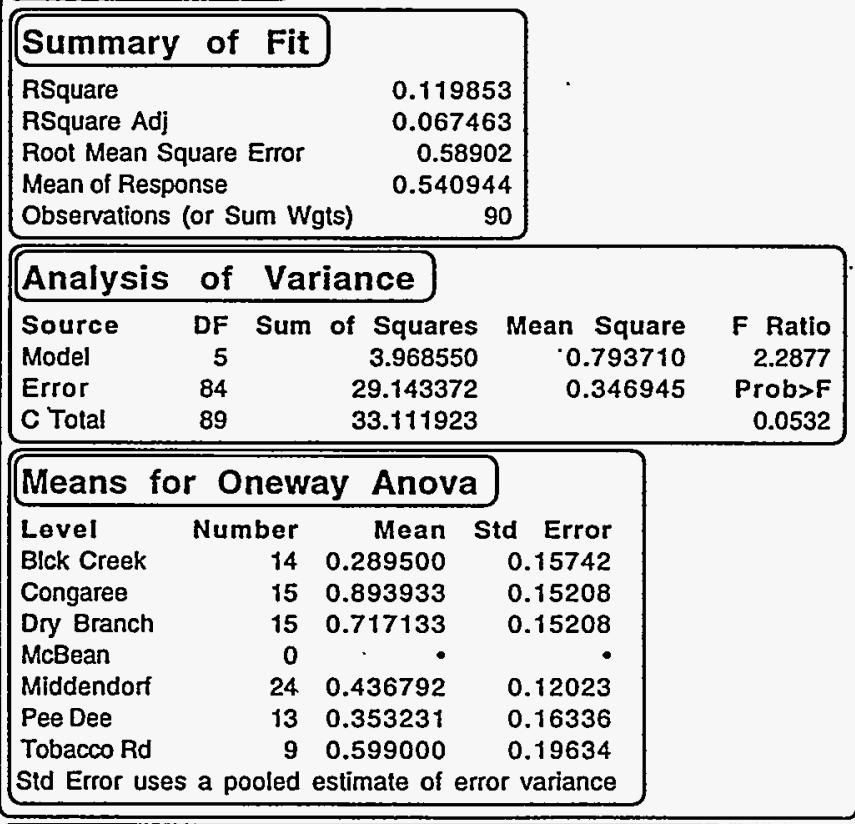

\section{Means Comparisons}$$
\text { Dif }=\text { Mean[i]-Mean[i] }
$$

Congaree

Dry Branch

Blck Creek

McBean

Tobacco Rd

Middendorf

Pee Dee

Alpha $=0.05$

Comparisons for each pair using Student's t

$\begin{array}{rrr}\text { Congaree } & \text { Dry Branch } & \text { Blck Creek } \\ 0.000000 & 0.176800 & 0.604433 \\ -0.1768 & 0.000000 & 0.427633 \\ -0.60443 & -0.42763 & 0.000000 \\ -0.29493 & -0.11813 & 0.309500 \\ -0.45714 & -0.28034 & 0.147292 \\ -0.5407 & -0.3639 & 0.063731\end{array}$

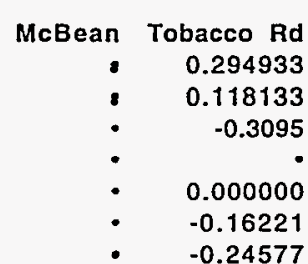

Middendorf

0.457142

0.280342

$-0.14729$

0.162208

0.000000

$-0.08356$
Pee Dee 0.540703 0.363903 $-0.06373$

0.245769 0.083561 0.000000
1.98862

Abs(Dif)-LSD

Congaree

Dry Branch

Blck Creek

McBean

Tobacco Rd

Middendort

Pee Dee $\begin{array}{lll}-0.42771 & -0.25091 & 0.169151\end{array}$

$-0.25091$

0.169151

$-0.19895$

0.071608

0.096845
$-0.42771$

$-0.0076$

$-0.44272$

$-0.07995$

$-0.19095$

$-0.24662$

$-0.38743$
$-0.10519$
McBean Tobacco Rd Middendorf

0.096845

$-0.10519 \quad-0.07995$

$-0.24662-0.38743$

$-0.29563-0.26216$

$-0.33814-0.31981$

$-0.31981 \quad-0.45944$

Positive values show pairs of means that are significantly different. 
FIGURE $9 x$.

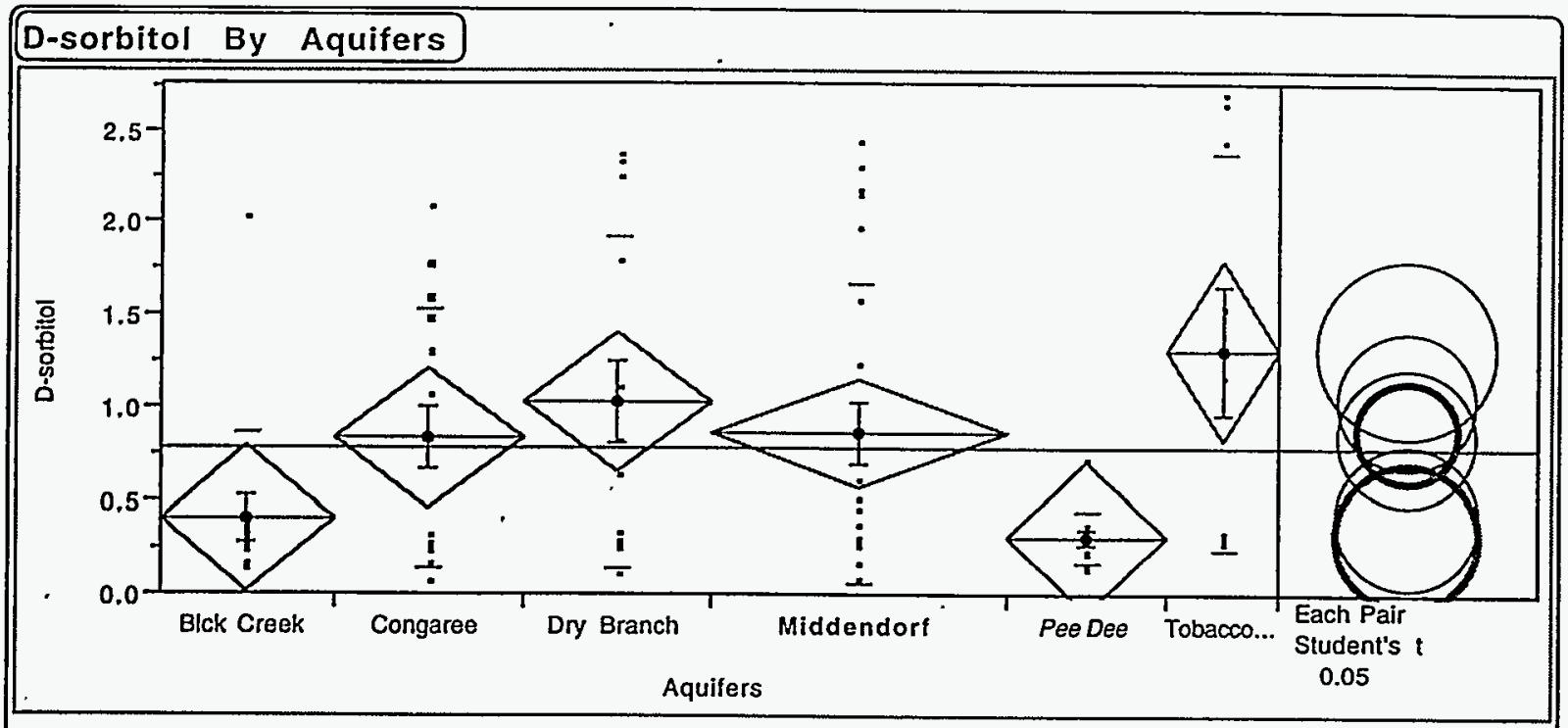

\title{
Oneway Anova
}

Summary of Fit

RSquare

RSquare Ad]

Root Mean Square Error

Mean of Response

Observations (or Sum Wgts)

\author{
0.155564 \\ 0.1053 \\ 0.735583 \\ 0.779467 \\ 90
}

\begin{tabular}{|c|c|c|c|c|c|c|}
\hline \multicolumn{7}{|c|}{ Analysis of Variance } \\
\hline $\begin{array}{l}\text { Source } \\
\text { Model } \\
\text { Error } \\
\text { C Total } \\
\end{array}$ & $\begin{array}{r}\text { DF } \\
5 \\
84 \\
89 \\
\end{array}$ & Sum & $\begin{array}{r}\text { of Squares } \\
8.373075 \\
45.450902 \\
53.823976 \\
\end{array}$ & Mean & $\begin{array}{l}\text { Square } \\
1.67461 \\
0.54108\end{array}$ & $\begin{array}{r}\text { F Ratio } \\
3.0949 \\
\text { Prob }>F \\
0.0130 \\
\end{array}$ \\
\hline \multicolumn{7}{|c|}{ Means for Oneway Anova } \\
\hline $\begin{array}{l}\text { Level } \\
\text { Blck Creek } \\
\text { Congaree } \\
\text { Dry Branch } \\
\text { McBean } \\
\text { Middendorf } \\
\text { Pee Dee } \\
\text { Tobacco Rd } \\
\text { Std Error use }\end{array}$ & $\begin{array}{c}\text { Num } \\
\text {. }\end{array}$ & $\begin{array}{r}\text { nber } \\
14 \\
15 \\
15 \\
0 \\
24 \\
13 \\
9 \\
\text { goled }\end{array}$ & $\begin{array}{r}\text { Mean } \mathrm{s} \\
0.41293 \\
0.83153 \\
1.03093 \\
0 . \\
0.85933 \\
0.31146 \\
1.30678 \\
\text { sstimate of err }\end{array}$ & $\begin{array}{r}\text { Std Err } \\
0.196 \\
0.189 \\
0.189 \\
\\
0.150 \\
0.204 \\
0.245 \\
\text { ror varian }\end{array}$ & $\begin{array}{r}\text { ror } \\
59 \\
93 \\
93 \\
15 \\
15 \\
01 \\
19 \\
\text { nce }\end{array}$ & \\
\hline
\end{tabular}

\section{Means Comparisons}

\begin{tabular}{|c|c|c|c|c|c|c|c|}
\hline $\begin{array}{l}\text { Dif=Mean[l]-Mean }[\}] \\
\text { Dry Branch }\end{array}$ & $\begin{array}{r}\text { Dry Branch } \\
0.000000\end{array}$ & $\begin{array}{r}\text { Middendorf } \\
0.171600\end{array}$ & $\begin{array}{r}\text { Blck Creek } \\
0.618005\end{array}$ & McBean & $\begin{array}{r}\text { Tobacco Rd } \\
-0.27584\end{array}$ & $\begin{array}{r}\text { Congaree } \\
0.199400\end{array}$ & $\begin{array}{l}\text { Pee Dee } \\
0.719472\end{array}$ \\
\hline Mlddendorf & -0.1716 & 0.000000 & 0.446405 & - & -0.44744 & 0.027800 & 0.547872 \\
\hline Blck Creek & -0.618 & -0.4464 & 0.000000 & $\bullet$ & -0.89385 & -0.4186 & 0.101467 \\
\hline McBean & $\bullet$ & - & - & - & $\bullet$ & - & $\bullet$ \\
\hline Tobacco Rd & 0.275844 & 0.447444 & 0.893849 & - & 0.000000 & 0.475244 & 0.995316 \\
\hline Congaree & -0.1994 & -0.0278 & 0.418605 & $\bullet$ & -0.47524 & 0.000000 & 0.520072 \\
\hline Pee Dee & -0.71947 & -0.54787 & -0.10147 & - & -0.99532 & -0.52007 & 0.000000 \\
\hline
\end{tabular}

Alpha $=0.05$

Comparisons for each pair using Student's t

$$
t
$$

1,98862

Abs(DIf)-LSD

Dry Branch

Dry Branch

Branch
-0.53414
-0.30986
0.074414
Middendorf Blck Creek $-0.30986$
$-0.42227$
$-0.04553$
0.074414
$-0.04553$
$-0.12431$
$-0.45366$
0.044132
$-0.55288$

Blck Creck

0.074414

McBean

Tobacco Rd

$-0.34092$

$-0.33474$

Poo Deo

0.165172

Posilive values show pairs of means that are significantly different.

Pee Dee

0.165172

0.044132

.0 .46195

0.361006

$-0.03423$

$-0.57375$ 
FIGURE $9 y$.

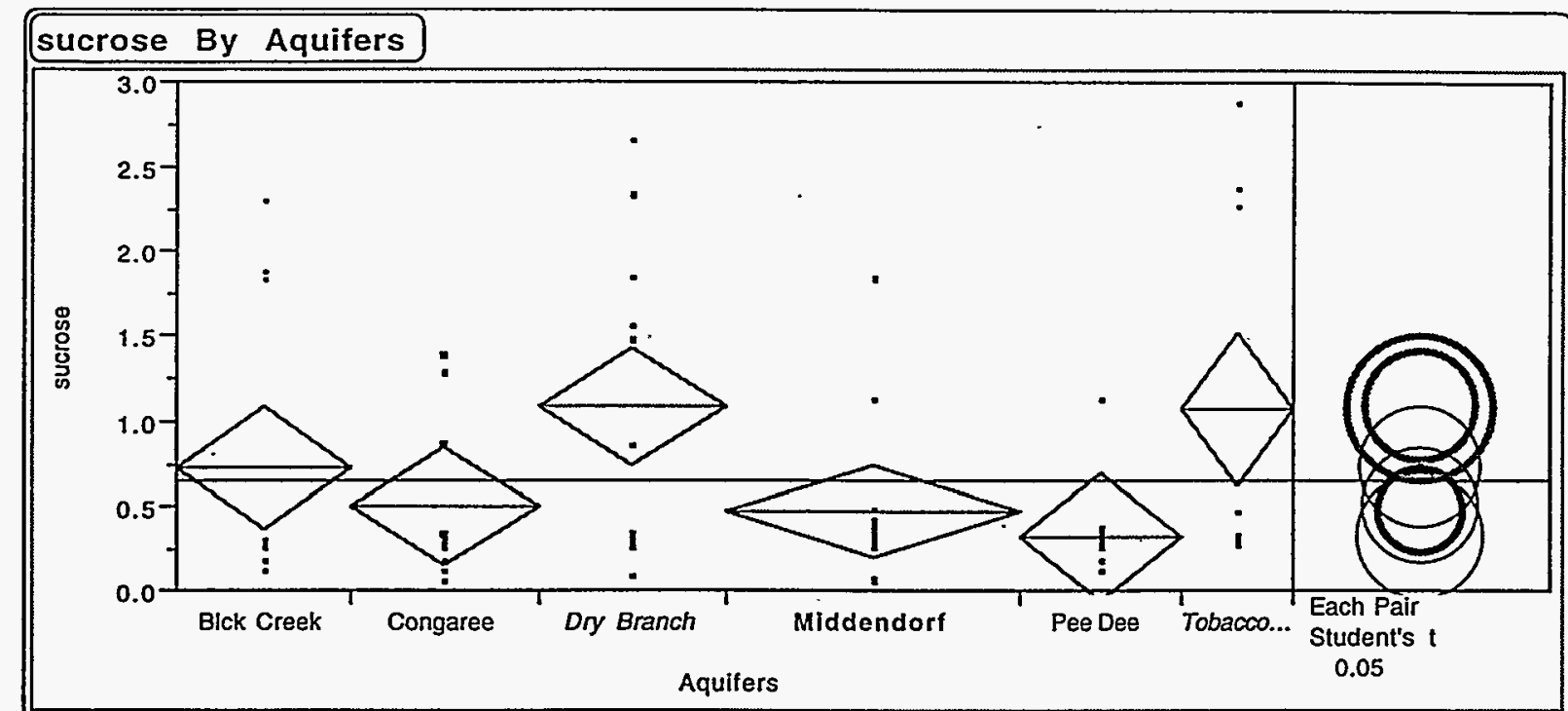

\section{Oneway Anova}

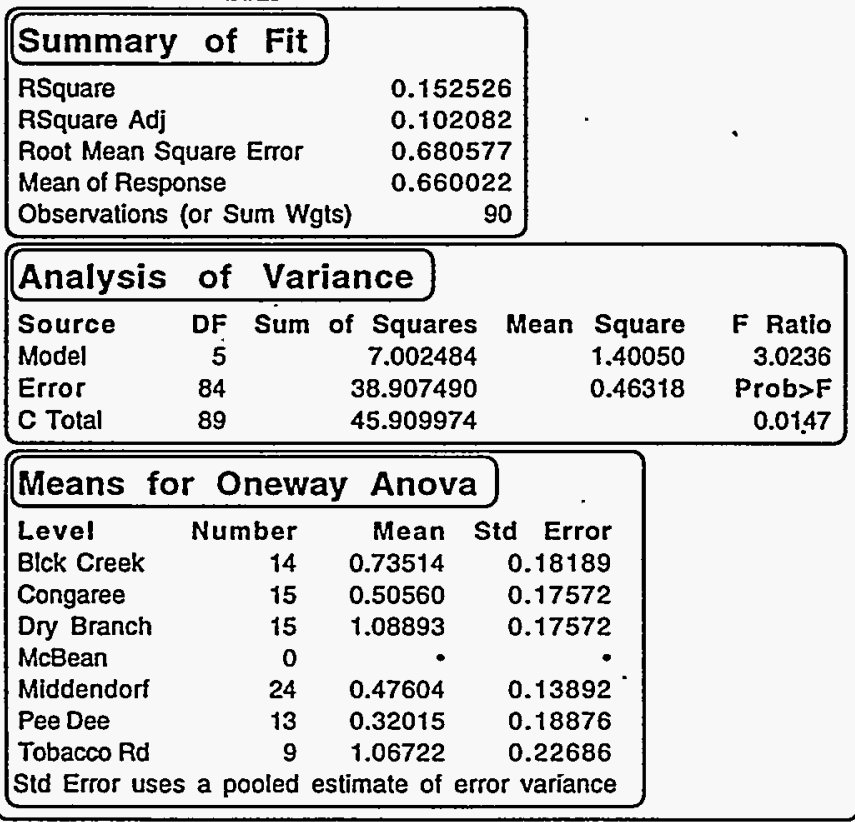

\section{Means Comparisons}

Dif=Mean[i]-Mean[j] Dry Branch

Dry Branch

Blck Creek

Congaree

McBean

Tobacco Rd

Middendorf

Pee Dee

Alpha $=0.05$

Comparisons for each pair using Student's t

$$
\begin{array}{rrr}
\text { Branch } & \text { Blck Creek } & \text { Congaree } \\
0.000000 & 0.353790 & 0.583333 \\
-0.35379 & 0.000000 & 0.229543 \\
-0.58333 & -0.22954 & 0.000000 \\
-0.02171 & 0.332079 & 0.561622 \\
-0.61289 & -0.2591 & -0.02956 \\
-0.76878 & -0.41499 & -0.18545
\end{array}
$$

\section{McBean Tobacco Rd Middendorf 0.612892 \\ 0.259101 \\ 0.029558 \\ 0.591181 \\ 0.000000 \\ $-0.15589$ \\ Pee Dee 0.768779 0.414989 0.185446 \\ 0.747068 0.155888 0.000000}

1.98862

Abs(Dif)-LSD

Dry Branch

Blck Creek

Congaree

McBean

Tobacco Rd

Middendorf

Pee Dee
Dry Branch Blck Creek Congaree
$-0.49419$
$-0.14915$
0.089139
$-0.54894$
0.167431
0.255930

McBean Tobacco Rd Middendorf Pee Dee

$0.167431 \quad 0.255930$

$-0.19604 \quad-0.1063$

$-0.4159-0.3274$

$0.062177 \quad 0.160192$

$-0.39069-0.31018$

$\begin{array}{ll}-0.31018 & -0.53085\end{array}$

Positive values show pairs of means that are significantly different. 
FIGURE $9 z$.

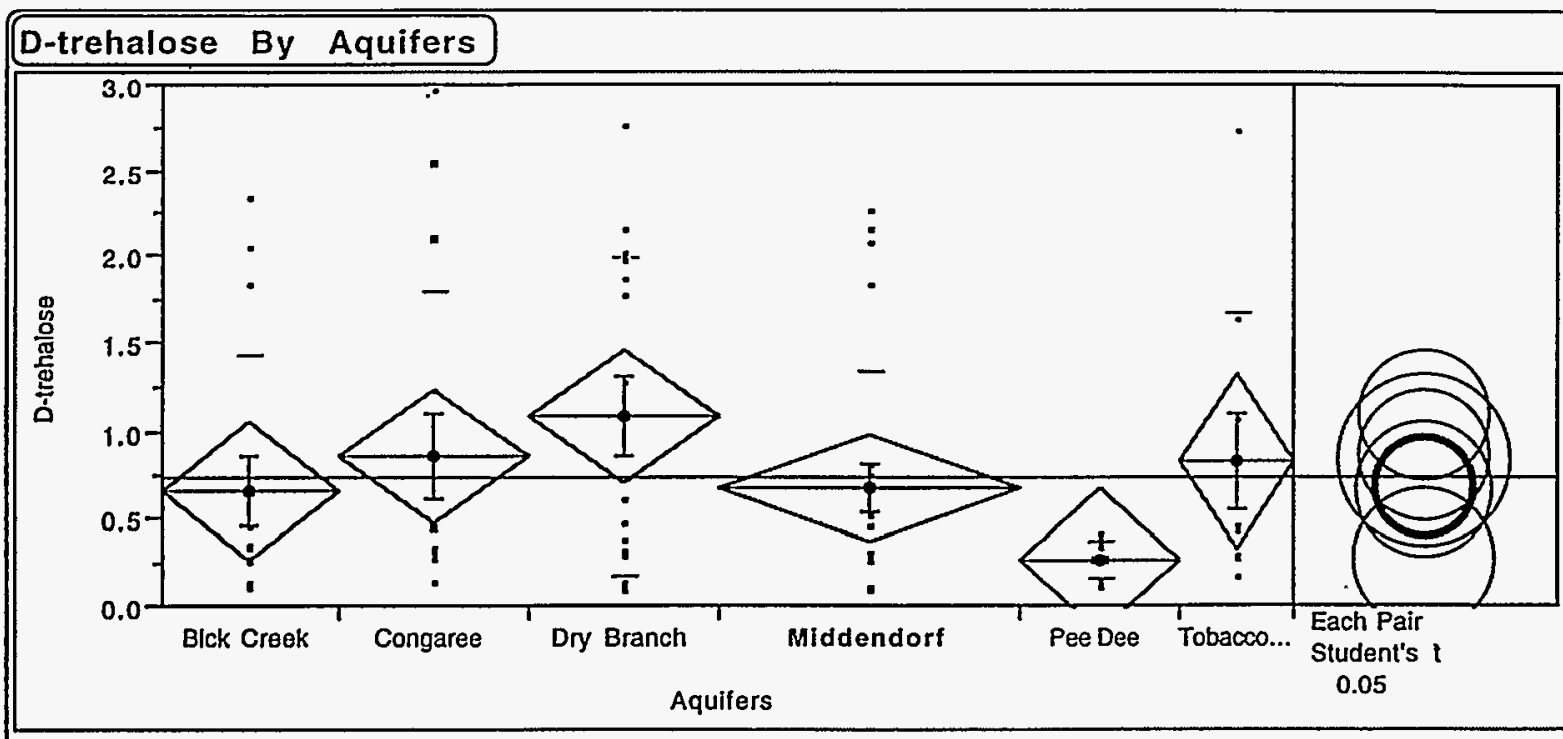

\section{Oneway Anova}

Summary of Fit

RSquare

RSquare Adj

Root Mean Square Error

Mean of Response

Observations (or Sum Wgts)

0.095861
0.042043
0.762667
0.728989
90

\begin{tabular}{|c|c|c|c|c|c|}
\hline \multicolumn{6}{|c|}{ Analysis of Variance } \\
\hline $\begin{array}{l}\text { Source } \\
\text { Model } \\
\text { Error } \\
\text { C Total }\end{array}$ & Sum & $\begin{array}{r}\text { of Squares } \\
5.180296 \\
48.859499 \\
54.039795\end{array}$ & Mean & $\begin{array}{l}\text { Square } \\
1.03606 \\
0.58166\end{array}$ & $\begin{array}{r}\text { F Ratio } \\
1.7812 \\
\text { Prob }>F \\
0.1255\end{array}$ \\
\hline \multicolumn{6}{|c|}{ Means for Oneway Anova } \\
\hline $\begin{array}{l}\text { Level } \\
\text { Blck Creek } \\
\text { Congaree } \\
\text { Dry Branch } \\
\text { McBean } \\
\text { Middendort } \\
\text { Pee Dee } \\
\text { Tobacco Rd } \\
\text { Std Error uses a p }\end{array}$ & $\begin{array}{r}\text { nber } \\
14 \\
15 \\
15 \\
0 \\
24 \\
13 \\
9 \\
\text { ooled e }\end{array}$ & $\begin{array}{c}\text { Mean S } \\
0.65929 \\
0.86187 \\
1.09007 \\
\text {. } \\
0.67546 \\
0.26931 \\
0.82089 \\
\text { stimate of err }\end{array}$ & $\begin{array}{l}\text { Std Err } \\
0.203 \\
0.196 \\
0.196 \\
\\
0.155 \\
0.211 \\
0.254 \\
\text { ror variar }\end{array}$ & $\begin{array}{r}\text { ror } \\
83 \\
92 \\
92 \\
\text { - } \\
68 \\
53 \\
22 \\
\text { ince }\end{array}$ & \\
\hline
\end{tabular}

\section{Means Comparisons}

\begin{tabular}{|c|c|c|c|c|c|c|c|}
\hline $\begin{array}{l}\text { Dif=Mean }[I]-\text { Mean }[J] \\
\text { Dry Branch }\end{array}$ & $\begin{array}{r}\text { Dry Branch } \\
0.000000\end{array}$ & $\begin{array}{r}\text { Congaree } \\
0.228200\end{array}$ & $\begin{array}{r}\text { Blck Creek } \\
0.430781\end{array}$ & McBean & $\begin{array}{r}\text { Tobacco Rd } \\
0.269178\end{array}$ & $\begin{array}{r}\text { Middendorf } \\
0.414608\end{array}$ & $\begin{array}{r}\text { Pee Dee } \\
0.820759\end{array}$ \\
\hline Congaree & -0.2282 & 0.000000 & 0.202581 & 8 & 0.040978 & 0.186408 & 0.592559 \\
\hline Blck Creek & -0.43078 & -0.20258 & 0.000000 & - & -0.1616 & -0.01617 & 0.389978 \\
\hline McBean & • & - & - & - & - & - & - \\
\hline Tobacco Rd & -0.26918 & -0.04098 & 0.161603 & - & 0.000000 & 0.145431 & 0.551581 \\
\hline Middendorf & -0.41461 & -0.18641 & 0.016173 & - & -0.14543 & 0.000000 & 0.406151 \\
\hline Pee Deo & -0.82076 & -0.59256 & -0.38998 & - & -0.55158 & -0.40615 & 0.000000 \\
\hline
\end{tabular}

Alpha $=0.05$

Comparisons for each pair using Student's $t$ $t$ 1.98862

Abs(DIf)-LSD

Dry Branch

Congaree

Blck Croek

McBean

Tobacco Rd

Middendorf

Pee Dee
Dry Branch
$-0.5538$
$-0.3256$
$-0.13283$
$-0.3703$
$-0.08458$

0.246050

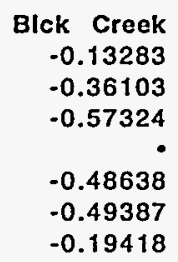

McBean

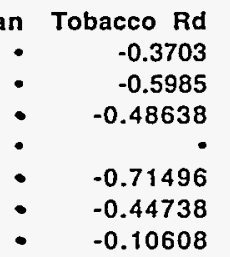

Middendorf

$\begin{array}{ll}0.08458 & 0.246050\end{array}$

$\begin{array}{ll}-0.31278 & 0.017850\end{array}$

$\begin{array}{ll}-0.49387 & -0.19418\end{array}$

$\begin{array}{ll}-0.44738 & -0.10608\end{array}$

$-0.43782 \quad-0.11614$

$\begin{array}{ll}-0.11614 & -0.59488\end{array}$

Posillve values show pairs of means that are significantly different. 
FIGURE 9aa.

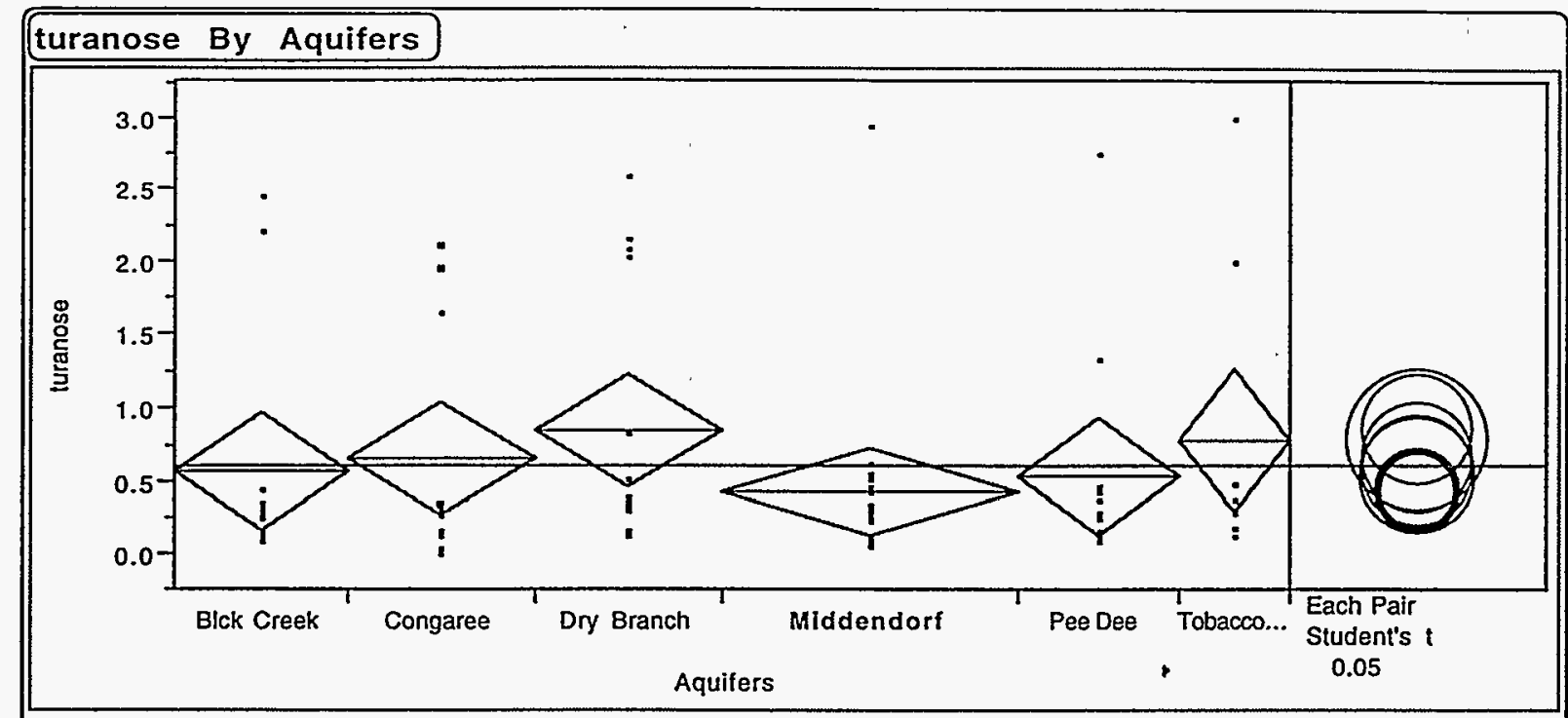

\section{Oneway Anova}

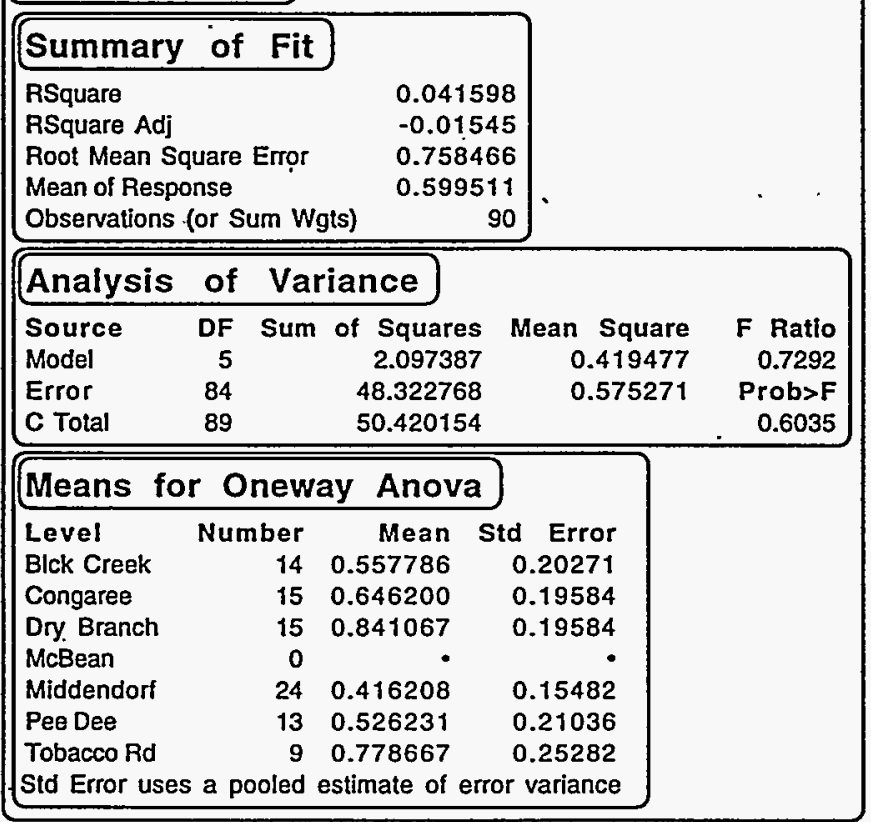

\section{Means Comparisons}

Dif=Mean[i]-Mean[j] Dry Branch Congaree Blck Creek Dry Branch

Congaree

Blck Creek

McBean

Tobacco Rd

Pee Dee

0.000000

\section{McBean Tobacco Rd}

Pee Dee

Middendorf

$\begin{array}{lll}-0.19487 & 0.000000 & 0.088414\end{array}$

$\begin{array}{lll}-0.28328 & -0.08841 & 0.000000\end{array}$

$\begin{array}{lll}-0.0624 & 0.132467 & 0.220881\end{array}$

$\begin{array}{lll}-0.31484 & -0.11997 & -0.03155\end{array}$

$\begin{array}{lll}-0.42486 & -0.22999 & -0.14158\end{array}$

Middendorf

$\begin{array}{rrr}\text { - } & 0.062400 & 0.314836 \\ \text { - } & -0.13247 & 0.119969 \\ \text { - } & 0.22088 & 0.031555 \\ \text { - } & 0.000000 & 0.252436 \\ \text { - } & -0.25244 & 0.000000 \\ & -0.36246 & -0.11002\end{array}$

0.424858

0.229992

0.141577

0.362458

0.110022

0.000000

Alpha $=0.05$

Comparisons for each pair using Student's $t$

1.98862

Abs(Dit)-LSD

Dry Branch

Congaree

Blck Creek

McBean

Tobacco Rd

Pee Dee

Middendorf

Dry Branch

$-0.55075$

$-0.35589$

$-0.27722$

$-0.57355$

$-0.25671$

$-0.07158$

Congaree

$-0.35589$

$-0.55075$

$-0.47209$

$-0.50349$

$-0.45157$

$-0.26645$
Bick Creek

0.27722

$-0.47209$

$-0.57008$

$-0.42353$

$-0.54939$

.0 .36566
McBean Tobacco Rd

- -0.57355

- $\quad-0.50349$

- -0.42353

.

$-0.71102$

- $\quad-0.40161$

- $\quad-0.22709$

Pee Dee

$-0.25671$

$-0.45157$

$-0.54939$

$-0.40161$

$-0.5916$

$-0.40939$

Middendort

$-0.07158$

$-0.26645$

$-0.36566$

$-0.22709$

$-0.40939$

$-0.43541$

Positive values show pairs of means that are significantly different. 
FIGURE $9 \mathrm{bb}$.

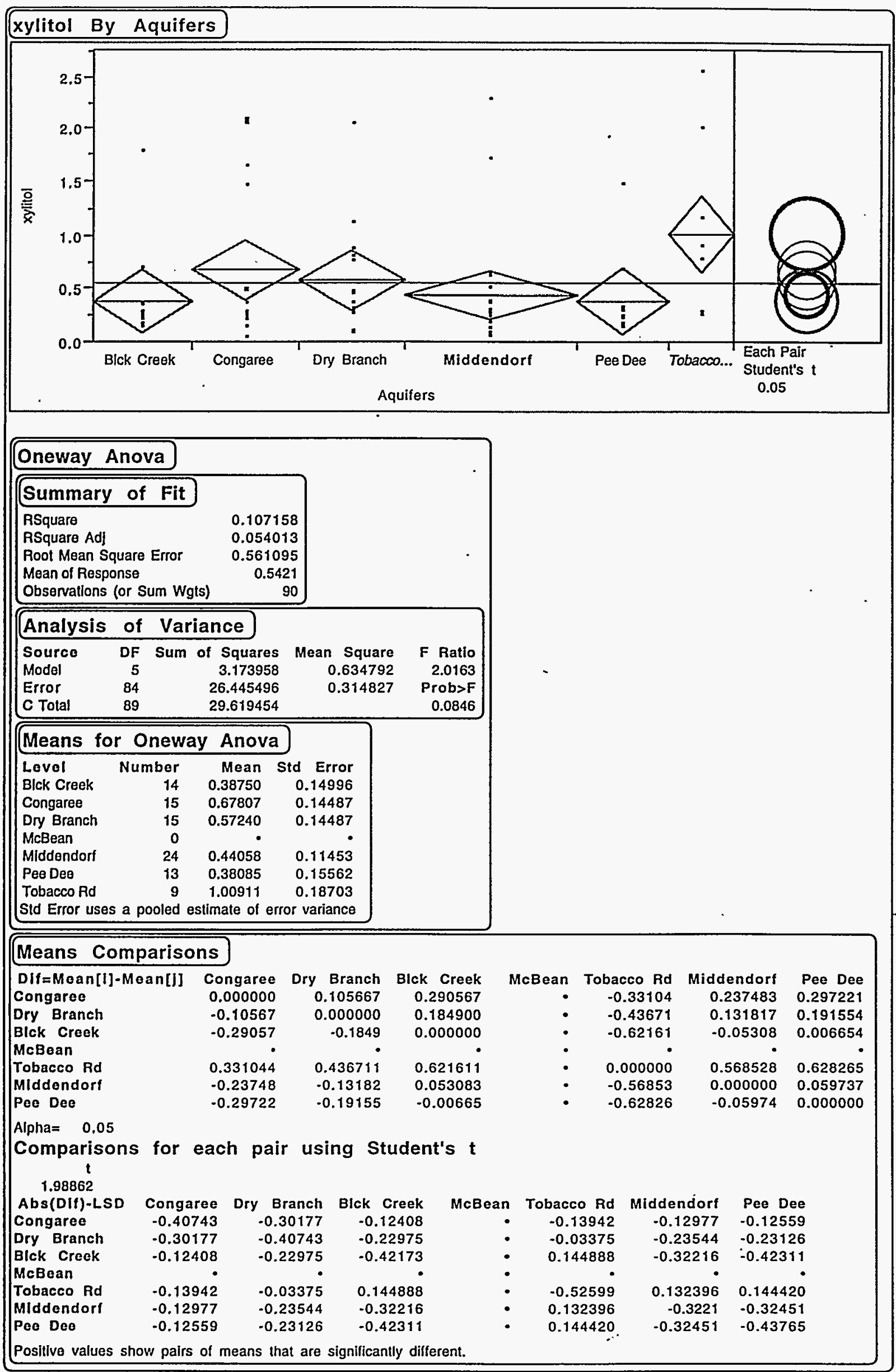


FIGURE 70 .

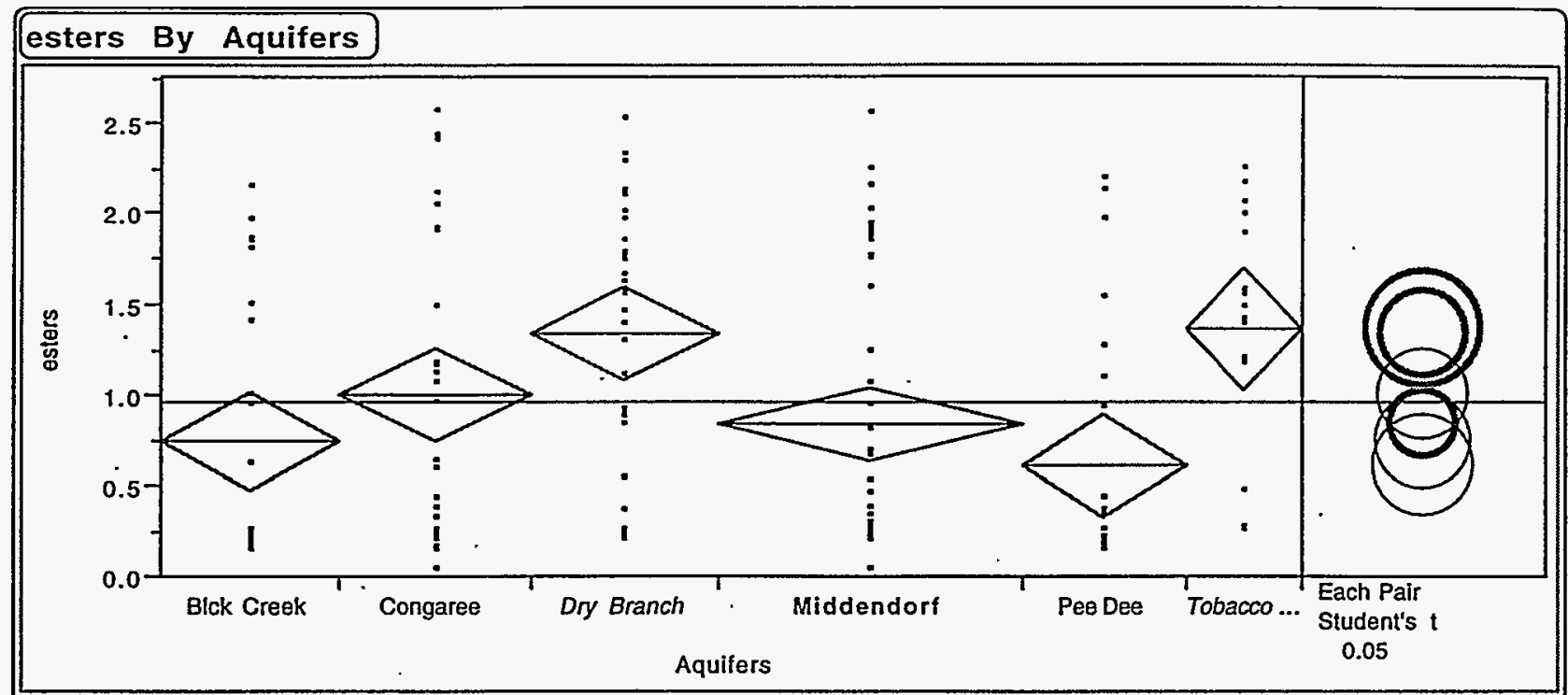

\section{Oneway Anova}

\section{Summary of Fit}

RSquare

0.116064

0.090664

RSquare Adj

0.730982

Root Mean Square Error

0.955928

Mean of Response

180

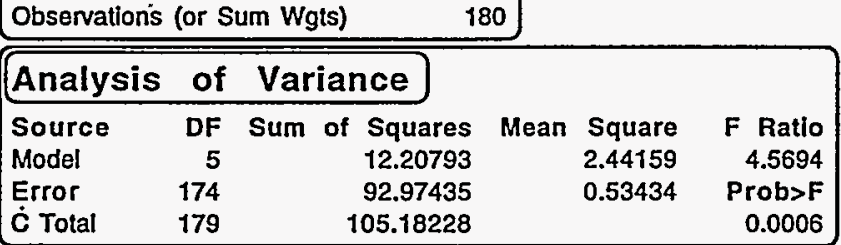

Means for Oneway Ánova

Level Number Mean Std Error

Blck Creek · $\quad 28 \quad 0.74868 \quad 0.13814$

Congaree : . $\quad 30 \quad 1.00467 \quad 0.13346$

$\begin{array}{llll}\text { Dry Branch } & 30 & 1.33797 & 0.13346\end{array}$

McBean

Middendorf

Pee Dee

$\begin{array}{rr}0 & \\ 48 & 0.84388\end{array}$

0.10551

0.61208

0.14336

Tobacco Rd

1.35583

0.17229

Std Error uses a pooled estimate of error variance

\section{Means Comparisons}

$\begin{array}{lrrr}\text { Dif=Mean[I]-Mean[J] } & \text { Dry Branch } & \text { Congaree } & \text { Blck Creek } \\ \text { Dry Branch } & 0.000000 & 0.333300 & 0.589288 \\ \text { Congaree } & -0.3333 & 0.000000 & 0.255988 \\ \text { Blck Creek } & -0.58929 & -0.25599 & 0.000000 \\ \text { McBean } & - & \bullet & \\ \text { Tobacco Rd } & 0.017867 & 0.351167 & 0.607155 \\ \text { Middendorf } & -0.49409 & -0.16079 & 0.095196 \\ \text { Pee Dee } & -0.72589 & -0.39259 & -0.1366\end{array}$

$-0.72589$

$-0.39259$

$-0.1366$
McBean Tobacco Rd Middendorf $-0.01787$

$-0.35117$

$-0.60715$

0.000000

$-0.51196$

$-0.74376$

$$
\begin{array}{rr}
0.494092 & 0.725890 \\
0.160792 & 0.392590 \\
-0.0952 & 0.136602 \\
\hline & \\
0.511958 & 0.743756 \\
0.000000 & 0.231798 \\
-0.2318 & 0.000000
\end{array}
$$

Comparisons for each pair using Student's $t$

1.97371

Abs(Dif)-LSD

Dry Branch

Congaree

Blck Creek

McBean

Tobacco Rd

Middendorf

Pee Dee.

$\begin{array}{rrr}\text { Dry Branch } & \text { Congaree } & \text { Blck Creek } \\ -0.37252 & -0.03922 & 0.210178 \\ -0.03922 & -0.37252 & -0.12312 \\ 0.210178 & -0.12312 & -0.38559 \\ -0.41228 & -0.07898 & 0.171287 \\ 0.158310 & -0.17499 & -0.24789 \\ 0.339311 & 0.006011 & -0.25633\end{array}$

McBea

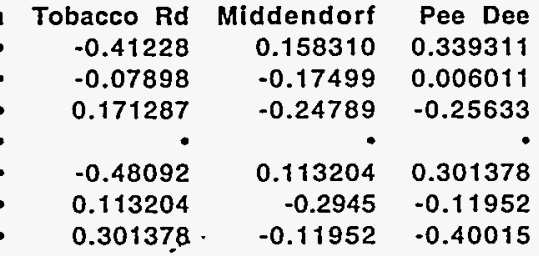


FIGURE $70 \mathrm{a}$.

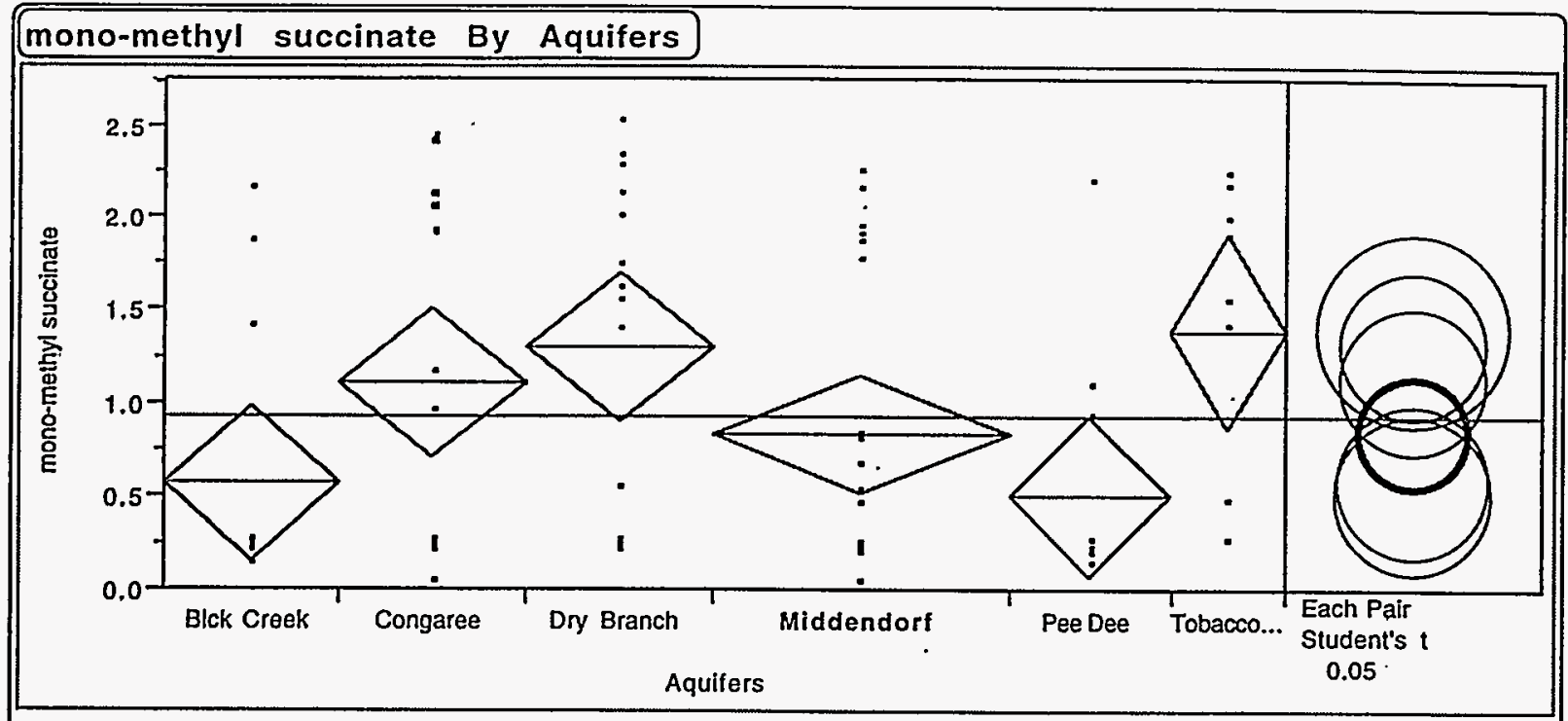

\section{Oneway Anova}

Summary of Fit

RSquare

RSquare Adj

Root Mean Square Error

Mean of Response

Observations (or Sum Wgts)

0.139297

0.088065

0.797599

0.922678

\section{Analysis of Variance}

\begin{tabular}{|lrrrrr|} 
Source & DF & Sum of Squares & Mean Square & F Ratio \\
Model & 5 & 8.648445 & 1.72969 & 2.7189 \\
Error & 84 & 53.437846 & 0.63616 & Prob $>$ F \\
C Total & 89 & 62.086292 & & 0.0251 \\
\hline
\end{tabular}

Means for Oneway Anova

Lovel Number Mean Std Error

$\begin{array}{llll}\text { Blck Creek } & 14 & 0.57371 & 0.21317\end{array}$

$\begin{array}{llll}\text { Congaree } & 15 & 1.10473 & 0.20594\end{array}$

Dry Branch

McBean

Middendorf

Pee Dee

Tobacco Rd

$15 \quad 1,29827$

0.83558

0.50208

0.20594

$\begin{array}{llll}13 & 9 & 1.37589 & 0.26587\end{array}$

Sid Error uses a pooled estimate of error variance

\section{Means Comparisons}

Dif=Mean[I]-Mean[J] Dry Branch

Dry Branch

Congaree

Blck Creek

McBean

Tobacco Rd

Middendorf

Pee Dee

Alpha $=0.05$

Comparisons for each pair using Student's $t$

1.98862

Abs(DIf)-LSD

Dry Branch

Congaree

Blck Creek

McBean

Tobacco Rd

Middendorf

Pee Dee

Dry Branch $-0.57917$

$-0.38564$

Congaree Blck Creek

.000000

$-0.19353$

$-0.72455$

0.077622

$-0.46268$

Congaree Blck Creek

0.193533

0.724552

0.531019

$-0.53102$

0.000000

0.271156

$-0.26915$

0.802175

0.261869

$-0.07164$
McBean Tobacco Rd Middendorf

0.462683

0.269150

$-0.26187$

0.540306

0.000000

$-0.33351$
Pee Dee

0.796190

0.602656

0.071637

0.873812

0.333506

0.000000 


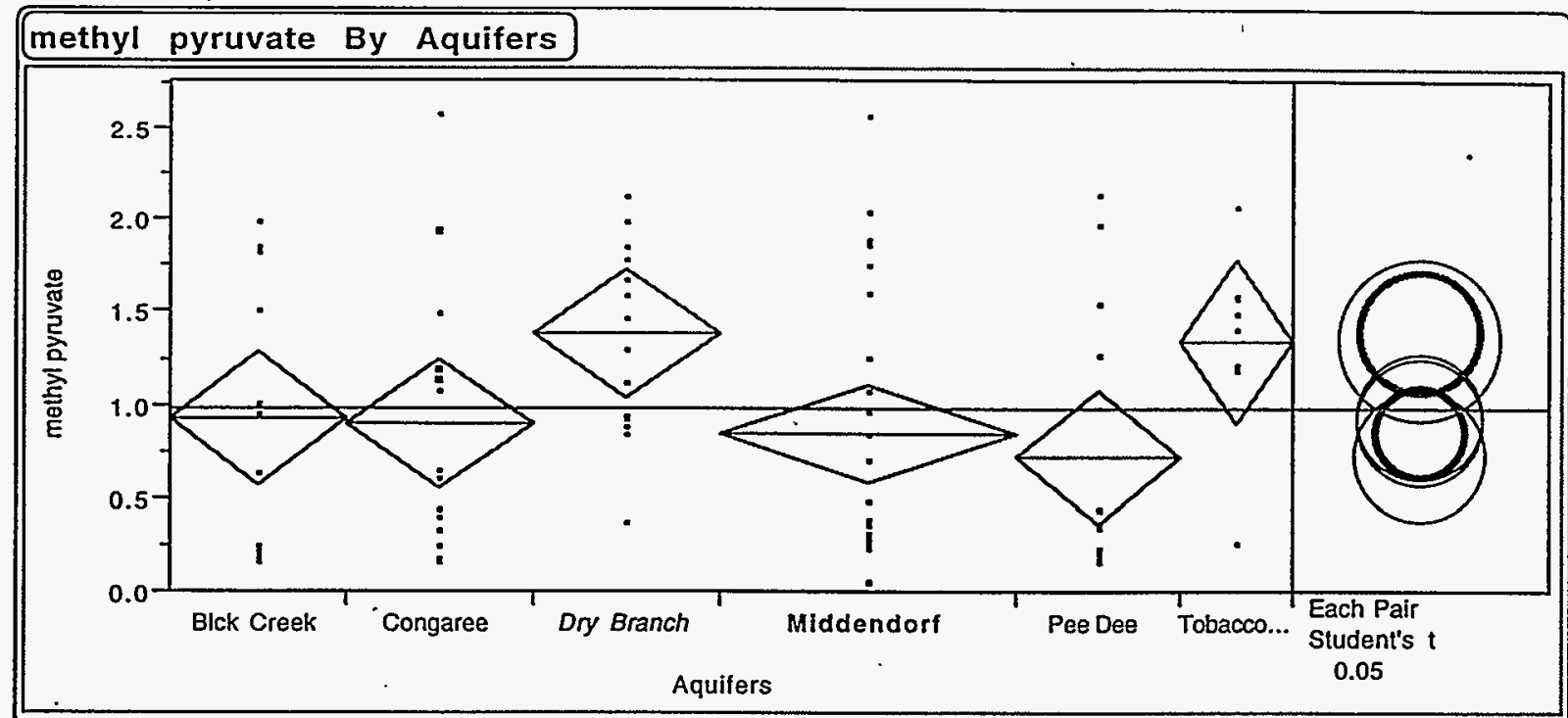

\section{Oneway Anova}

Summary of Fit

RSquare

0.114005

RSquare Adj

0.061267

Root Mean Square Error

0.672651

Mean of Response

0.989178

Observations (or Sum Wgts)

90

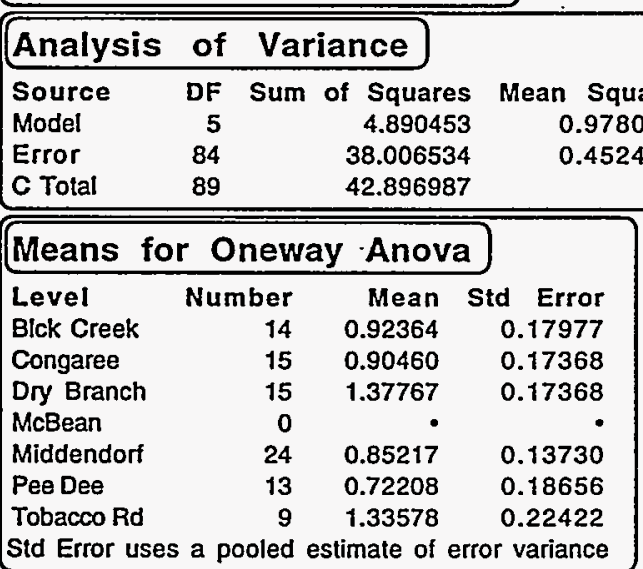

\section{Means Comparisons}

DIf=Mean[i]-Mean[j] Dry Branch

Dry Branch

Blck Creek

Congaree

McBean

Tobacco Rd

Middendorf

Pee Dee

Alpha $=0.05$

Comparisons for each pair using Student's t

$\begin{array}{rr}\text { Blck Creek } & \text { Congaree } \\ 0.454024 & 0.473067 \\ 0.000000 & 0.019043 \\ -0.01904 & 0.000000 \\ . & \\ 0.412135 & 0.431178 \\ -0.07148 & -0.05243 \\ -0.20157 & -0.18252\end{array}$

0.000000

$-0.45402$

$-0.47307$

.0 .04189

$-0.5255$

$-0.65559$
F Ratio

2.1617

Prob $>$ F 0.0660
1.98862

Abs(Dif)-LSD

Dry Branch

Blck Creek

Congaree

McBean

Tobacco Rd

Middendorf

Pee Dee
Dry Branch

$-0.04306$

$-0.01537$

$-0.52211$

0.085227

0.148713
Creek Congaree

$-0.04306$

$-0.50558$

$-0.47804$

$-0.15937$

$-0.37837$

$-0.31365$
$-0.01537$

$-0.47804$

$-0.48844$

$-0.13282$

$-0.38784$

$-0.32435$
McBean Tobacco Rd Middendorf - $\quad-0.52211 \quad 0.085227$

- $\quad-0.15937 \quad-0.37837$

$-0.13282$

- $\quad-0.63057$

$-0.63057$

$-0.03923$

0.033659
$-0.38784$

$-0.03923$

$-0.38614$

$-0.33055$
Pee Dee

0.148713

$-0.31365$

$-0.32435$

0.033659

$-0.33055$

$-0.52467$ 


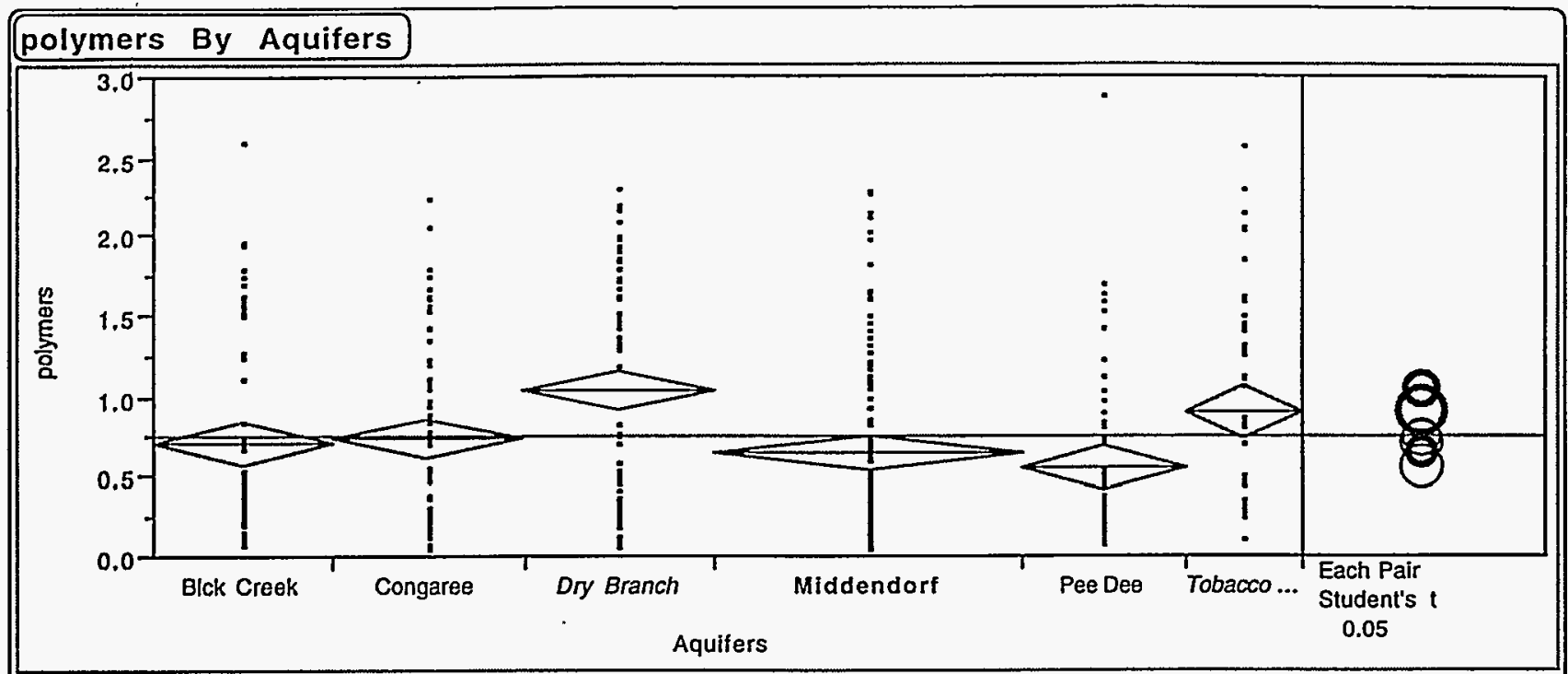

\section{Oneway Anova}

Summary of Fit

RSquare

RSquare Adj

Root Mean Square Error

Mean of Response

0.068679

0.058191

0.598172

0.744598

Observations (or Sum Wgts)

450

\begin{tabular}{||rrrrrr|}
\hline Analysis & of & Variance & & & \\
Source & DF & Sum of Squares & Mean Square & F Ratio \\
Model & 5 & 11.71549 & 2.34310 & 6.5484 \\
Error & 444 & 158.86777 & 0.35781 & Prob $>$ F \\
C Total & 449 & 170.58326 & & 0.0000 \\
\hline
\end{tabular}

Means for Oneway Anova

\begin{tabular}{lrrr}
\hline Level & Number & Mean & Std. Error \\
Blck Creek & 70 & 0.69791 & 0.07150 \\
Congaree & 75 & 0.73108 & 0.06907 \\
Dry Branch & 75 & 1.04011 & 0.06907 \\
McBean & 0 & - &. \\
Middendorf & 120 & 0.64218 & 0.05461 \\
Pee Dee & 65 & 0.54626 & 0.07419 \\
Tobacco Rd & 45 & 0.90682 & 0.08917
\end{tabular}

Std Error uses a pooled estimate of error variance

Means Comparisons

\begin{tabular}{|c|c|c|c|c|c|c|c|}
\hline Dif=Mean[i]-Mean[l] & $\begin{array}{l}\text { Dry Branch } \\
0,000000\end{array}$ & $\begin{array}{r}\text { Congaree } \\
0.309027\end{array}$ & $\begin{array}{r}\text { Blck Creek } \\
0.342192\end{array}$ & McBean & $\begin{array}{r}\text { Tobacco Rd } \\
0.133284\end{array}$ & $\begin{array}{r}\text { Middendorf } \\
0.397923\end{array}$ & $\begin{array}{l}\text { Pee Dee } \\
0.493845\end{array}$ \\
\hline Congaree & -0.30903 & 0.000000 & 0.033166 & - & -0.17574 & 0.088897 & 0.184818 \\
\hline Blck Creok & -0.34219 & -0.03317 & 0.000000 & - & -0.20891 & 0.055731 & 0.151653 \\
\hline McBean & 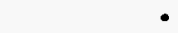 & . & & - & $\bullet$ & 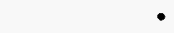 & \\
\hline Tobacco Rd & -0.13328 & 0.175742 & 0.208908 & - & 0.000000 & 0.264639 & 0.360561 \\
\hline Mlddendorf & -0.39792 & -0.0889 & -0.05573 & - & -0.26464 & 0.000000 & 0.095922 \\
\hline Pee Dee & -0.49385 & -0.18482 & -0.15165 & - & -0.36056 & -0.09592 & 0.000000 \\
\hline
\end{tabular}

Alpha $=0.05$

Comparisons for each pair using Student's $t$

$$
\text { t }
$$

Abs(DIf)-LSD

Dry Branch

Congaree

Blck Creek

McBean

Tobacco Rd

MIddendorf

Pee Dee
Dry Branch
$-0.19198$
0.117049
0.146817
$-0.08839$
0.224877
0.294620

Blck Creek

0.146817

$-0.16221$

$-0.19872$

$-0.01572$

$-0.12108$

$-0.05085$
McBean Tobacco Rd Mid -0.08839 Mddendorf $-0.04593$ $-0.01572$

$-0.24784$ 0.059139

0.132579
Pee Dee 0.2248770 .294620

$-0.08415 \quad-0.01441$

$-0.12108 \quad-0.05085$

$0.059139 \quad 0.132579$

$\begin{array}{ll}-0.15177 & -0.08513\end{array}$

$-0.08513-0.20622$

Positive values show pairs of means that are significantly different. 
FIGURE $77 \mathrm{a}$.

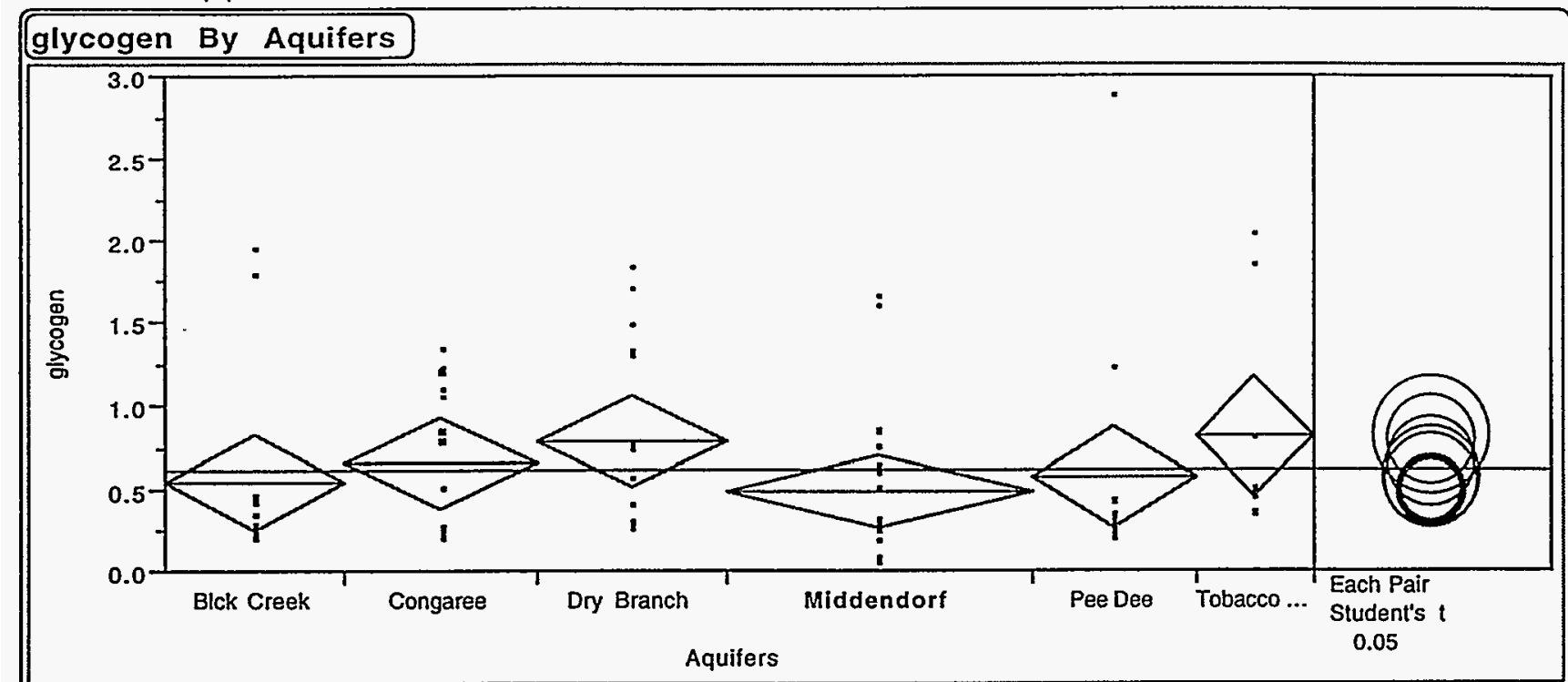

\section{Oneway Anova}

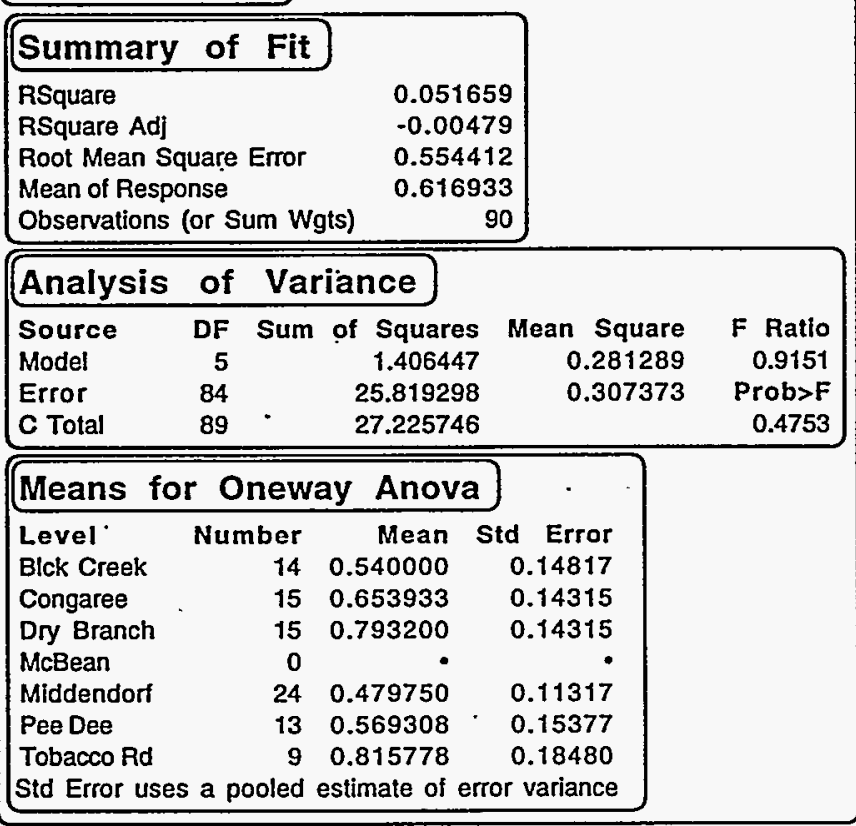

\section{Means Comparisons}

Dif=Mean[i]-Mean[j] Dry Branch

Dry Branch

Congaree

Blck Creek

McBean

Tobacco Rd

Pee Dee

Middendorf

0.000000

$-0.13927$

$-0.2532$

0.022578

$-0.22389$

$-0.31345$

Congaree Bick Creek

0.139267

0.000000

$-0.11393$

\subsection{0}

0.113933

0.000000

0.161844

$-0.08463$

$-0.17418$
0.275778

0.029308

$-0.06025$
McBean Tobacco Rd

$-0.02258$

$-0.16184$

$-0.27578$

0.000000

$-0.24647$

$-0.33603$
Pee Dee Middendorf

\subsection{0 .313450}

$\begin{array}{ll}0.084626 & 0.174183\end{array}$

$\begin{array}{ll}-0.02931 & 0.060250\end{array}$

0.246470

0.000000

$-0.08956$

\subsection{8}

0.089558

0.000000

Comparisons for each pair using Student's $t$

1.98862

Abs(Dif)-LSD

Dry Branch

Congaree

Blck Creek

McBean

Tobacco Rd

Pee Dee

Middendorf

Dry Branch

$-0.40258$

$-0.26331$

$-0.15651$

$-0.44228$

$-0.19389$

$-0.04943$

Congaree

$-0.26331$

Blck Creek

$-0.40258$

$-0.29577$

$-0.41671$

$-0.30302$

$-0.33315$

$-0.1887$

$-0.19527$

$-0.39534$

$-0.31052$
McBean Tobacco Rd

$-0.44228$

$-0.30302$

$-0.19527$

$-0.51973$

$-0.23161$

-0.0949 .1 .
Pee Dee

$-0.19389$

$-0.33315$

$-0.39534$

$-0.23161$

$-0.43244$

$-0.29011$
Middendorf $-0.04943$

$-0.1887$

$-0.31052$

$-0.09491$

$-0.29011$

$-0.31827$

Positive values show pairs of means that are significantly different. 
FIGURE $17 \mathrm{~b}$.

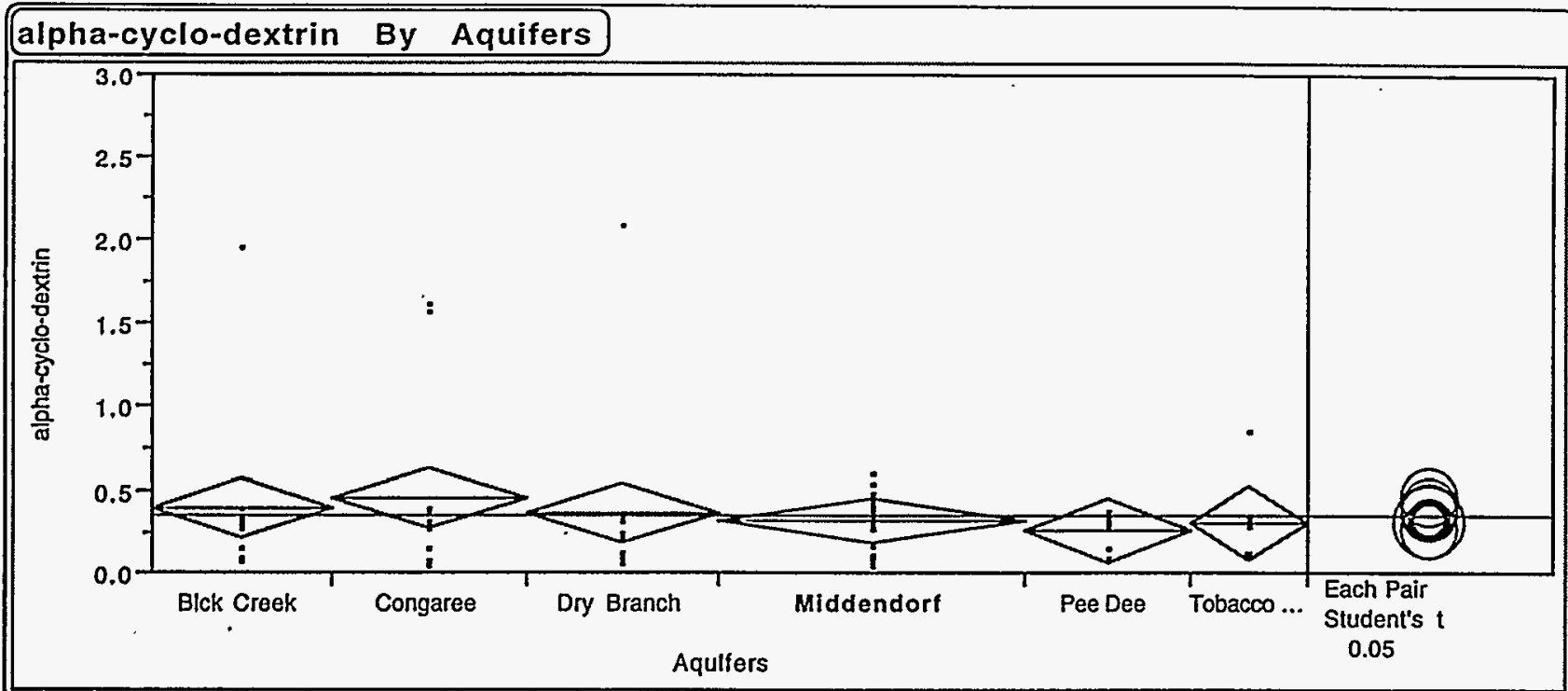

\section{Oneway. Anova}

\section{Summary of Fit}

RSquare

0.031521

$-0.02613$

RSquare Ad]

Root Mean Square Error

Mean of Response

0.34904

Observations (or Sum Wgts)

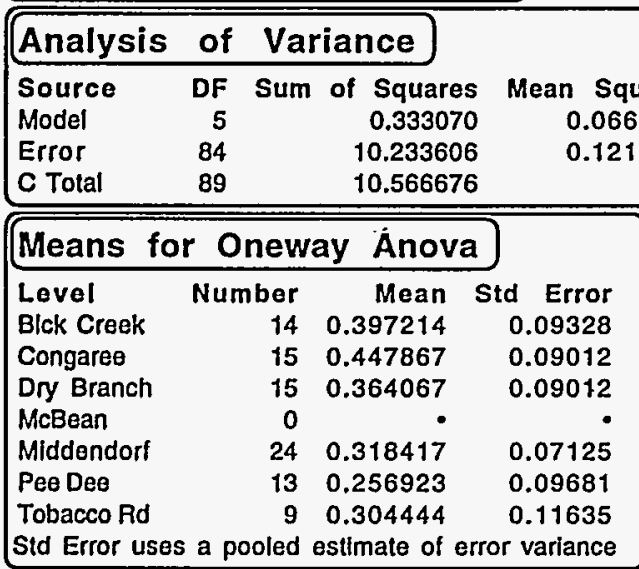

\section{Means Comparisons}

\begin{tabular}{|lrrr} 
Dlf=Mean[l]-Mean[I] & Congaree & Blck Creek & Dry Branch \\
Congaree & 0.000000 & 0.050652 & 0.083800 \\
Blck Creek & -0.05065 & 0.000000 & 0.033148 \\
Dry Branch & -0.0838 & -0.03315 & 0.000000 \\
McBean & - & - & \\
Middendorf & -0.12945 & -0.0788 & -0.04565 \\
Tobacco Rd & -0.14342 & -0.09277 & -0.05962 \\
Peo Deo & -0.19094 & -0.14029 & -0.10714 \\
& & &
\end{tabular}

McBean Middendorf Tobacco Rd Pee Dee - 0.129450

0.078798

0.045650

- 0.000000

-0.01397
$-\quad-0.06149$

$$
\begin{array}{rr}
0.143422 & 0.190944 \\
0.092770 & 0.140291 \\
0.059622 & 0.107144 \\
\hline & \\
0.013972 & 0.061494 \\
0.000000 & 0.047521 \\
-0.04752 & 0.000000
\end{array}
$$

Alpha $=0.05$

Comparisons for each pair using Student's $t$ $t$ 1.98862

Abs(DIf)-LSD

Congareo

Blck Creek

Dry Branch

McBean

Mlddendorf

Tobacco Rd

Pee Dee

$$
\begin{array}{r}
\text { Congaree } \\
-0.25345 \\
-0.20729 \\
-0.16965 \\
-0.09901 \\
-0.14924
\end{array}
$$

Blck Creek

$-0.20729$

ry Branch

$-0.16965$

McBean Middendorf Tobacco Rd

$-0.22479$

$-0.22479$

$-0.25345$

$-0.15463$

$-0.20378$

$-0.18281$

$-0.23304$

$-0.12705$

$-0.15588$

$-0.09901 \quad-0.14924$

$-0.15463-0.20378$

$-0.18281$

$-0.20037$

$-0.25733$

-0.17753 .

$-0.23304-0.15588$

$-0.25733$

$-0.25346$

Positive values show pairs of means that are significantly different. 


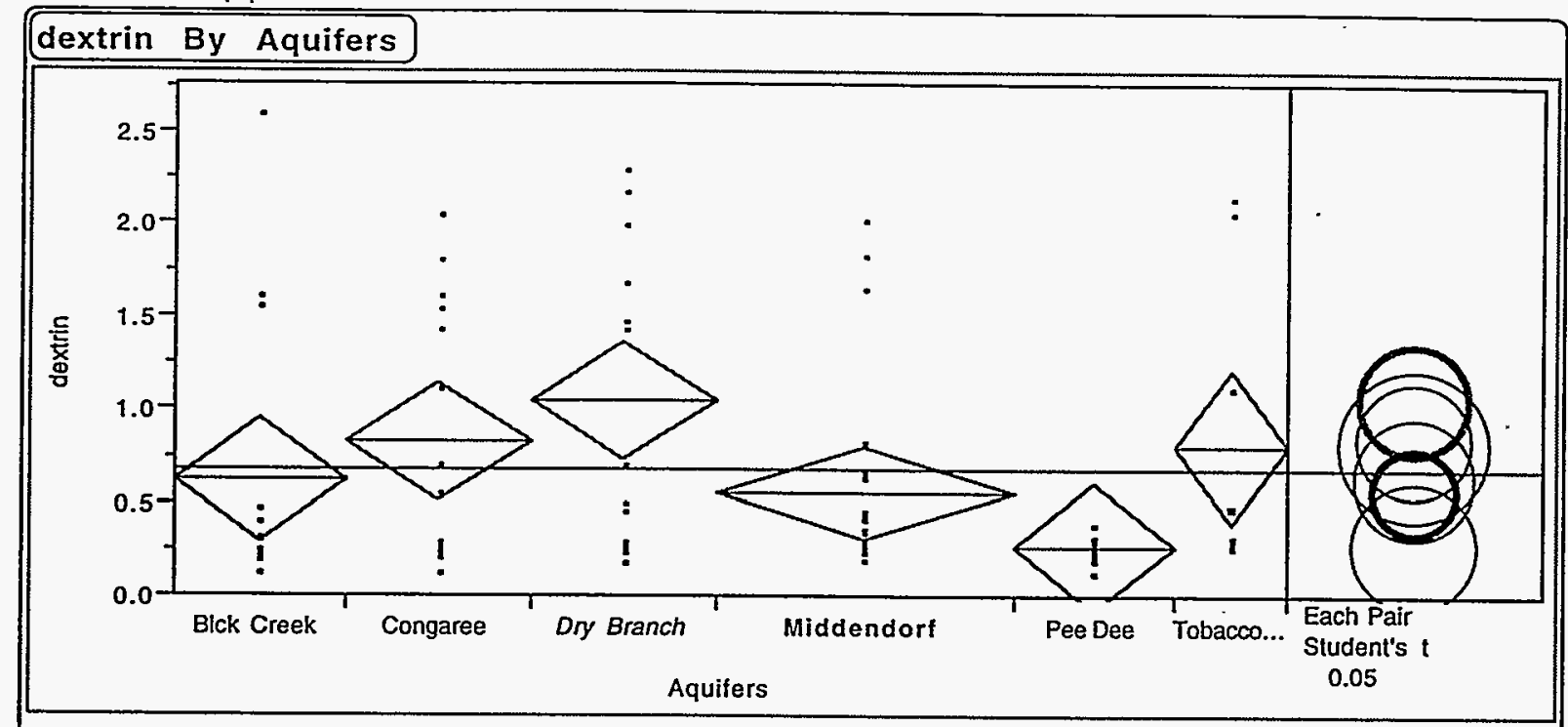

\section{Oneway Anova \\ Summary of Fit \\ RSquare}

RSquare Adj

Root Mean Square Error

Mean of Response

Observations (or Sum Wgts)

0.134728

0.083224

0.626902

0.674778

Analysis of Variance

\begin{tabular}{|lrrrrr}
\hline Source & DF & Sum of Squares & Mean Square & F Ratio \\
Model & 5 & 5.140248 & 1.02805 & 2.6159 \\
Error & 84 & 33.012531 & 0.39301 & Prob $>$ F \\
C Total & 89 & 38.152780 & & 0.0301 \\
\hline
\end{tabular}

Means for Oneway Anova

Level

Blck Creek

Congaree

Dry Branch

McBean

Middendorf

Pee Dee

Tobacco Rd

Number

Mean Std Error

140.62600

0.16755

$0.83220 \quad 0.16187$

1.04093

0.55333

0.16187

0.12797

0.17387

0.20897

Sid Error uses a pooled estimate of error variance

\section{Means Comparisons}

Dif=Mean[i]-Mean[i] Dry Branch Dry Branch

Congaree

Blck Creek

McBean

Tobacco Rd

Middendorf

Pee Dee

Alpha $=0.05$

Comparisons for each pair using Student's t

$$
\text { t }
$$

Abs(Dif)-LSD

Dry Branch

Congaree

Blck Creek

McBean

Tobacco Rd

Middendorf

Pee Dee

$\begin{array}{rr}\text { Branch } & \text { Congaree } \\ -0.45522 & -0.24649 \\ -0.24649 & -0.45522 \\ -0.04834 & -0.25708 \\ -0.28471 & -0.49344 \\ 0.077271 & -0.13146\end{array}$

$0.307838 \quad 0.099104$
Blck Creek

Congaree 0.208733

$-0.41493$

$-0.24093$

-0.4876
-0.78024

-0.0322
-0.27887

$-0.57151$
0.414933

0.206200

0.000000

0.174000

$-0.07267$

$-0.36531$
McBean Tobacco Rd Middendorf

- 0.240933

- 0.032200

- $\quad-0.174$

- $\quad-0.174$

- 0.000000

- $\quad 0.24667$

$-0.53931$
0.780241

$0.278867 \quad 0.571508$

0.072667

0.246667

0.000000

$-0.29264$
0.571508
0.365308

0.539308

0.292641

0.000000

Positive values show pairs of means that are significantly different. 
FIGURE 17d.

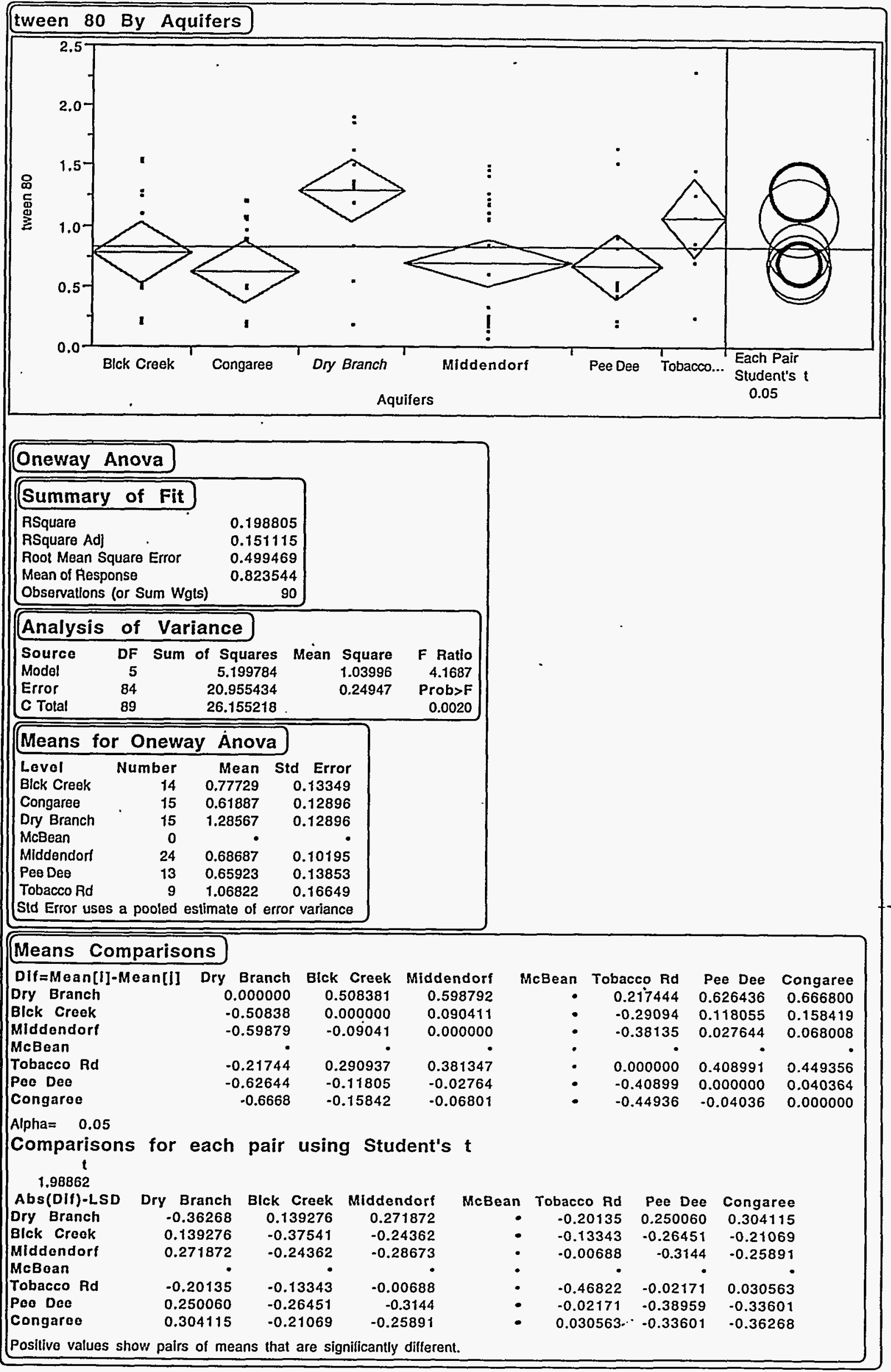


FIGURE 17e.

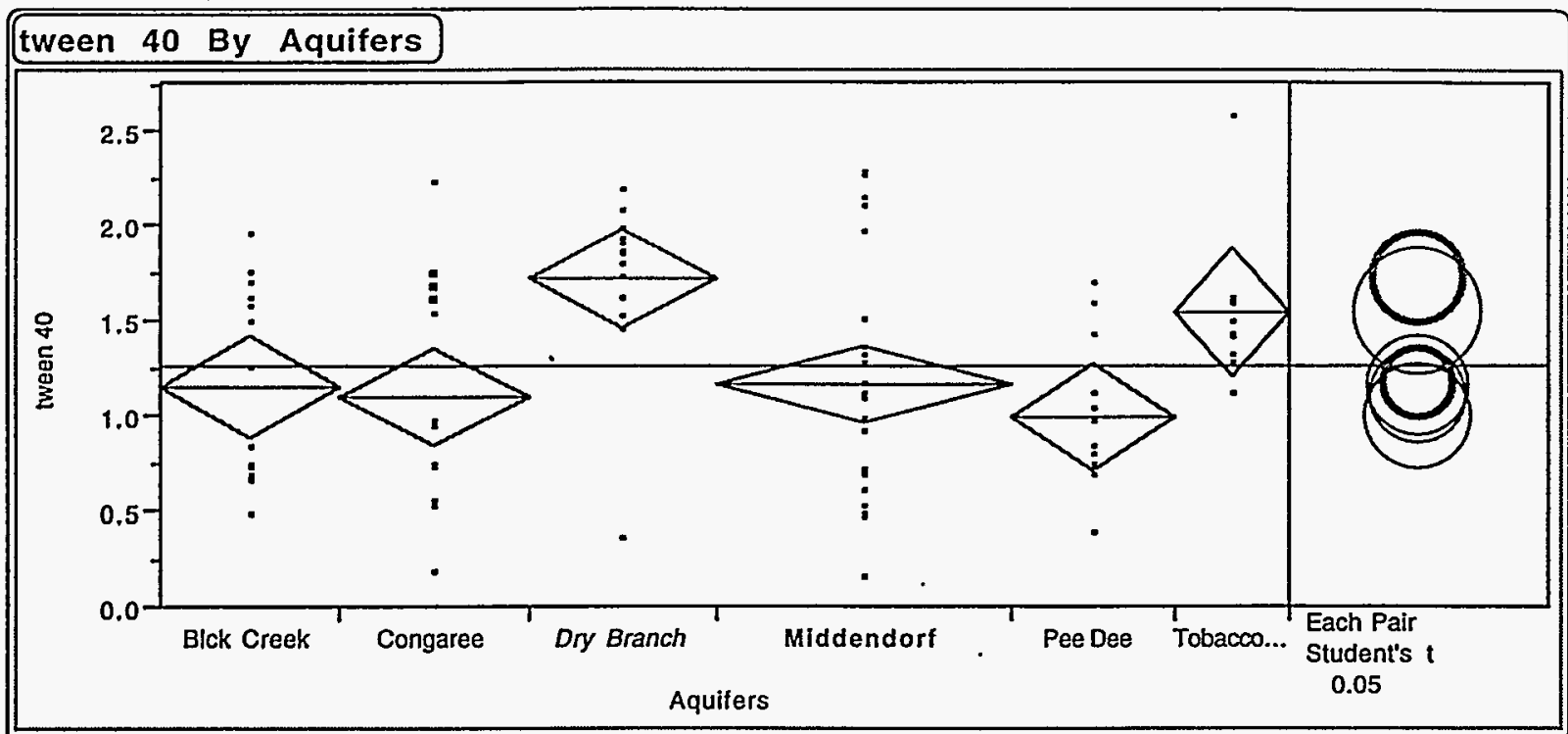

\section{Oneway Anova}

\section{Summarý of Fit}

RSquare

RSquare Adj

0.197341

Root Mean Square Error

Mean of Response

0.149564

0.51943

Observations (or Sum Wgts)

1.258156

\begin{tabular}{|lrrrrr}
\hline Analysis & of & \multicolumn{2}{c}{ Variance } & & \\
Source $_{\text {Model }}$ & DF & Sum of Squares & Mean Square & F Ratio \\
Error & 5 & 5.572118 & 1.11442 & 4.1304 \\
C Total & 84 & 22.663806 & 0.26981 & Prob $>$ F \\
\hline
\end{tabular}

Means for Oneway Ánova

Level Number " Mean Std Erro

$\begin{array}{llll}\text { Blck Creek } & 14 & 1.14907 & 0.13882\end{array}$

$\begin{array}{llll}\text { Congaree } & 15 & 1.10253 & 0.13412\end{array}$

Dry Branch

McBean

Middendorf

Pee Dee

Tobacco Rd

$15 \quad 1.71667$

1.17254

0.98515

1.54567

0.13412

Std Error uses a pooled estimate of error variance

\section{Means Comparisons}

Dif=Mean[i]-Mean[j] Dry Branch

Dry Branch

Middendor

Blck Creek

MeBean

Tobacco Rd

Congaree

Pee Dee

Alpha $=0.05$

Comparisons for each pair using Student's $t$

$\begin{array}{rrr}0.000000 & 0.544125 & 0.567595 \\ -0.54412 & 0.000000 & 0.023470 \\ -0.5676 & -0.02347 & 0.000000 \\ -0.171 & 0.373125 & 0.396595 \\ -0.61413 & -0.07001 & -0.04654 \\ -0.73151 & -0.18739 & -0.16392\end{array}$

McBean Tobacco Rd

0.171000

$-0.37312$

$-0.3966$

0.000000

$-0.44313$

$-0.56051$
Congaree

0.614133

0.070008

0.046538

0.443133

0.000000

$-0.11738$
Pee Dee

0.731513

0.187388

0.163918

0.560513

0.117379 0.000000
1.98862

Abs(Dif)-LSD

Dry Branch

Mlddendor

Blck Creek

McBean

Tobacco Rd

Congaree

Peo Dee

Dry Branch

$-0.37718$

0.204140

0.183740

$-0.26453$

0.236954

0.340096
Middendorf Blck Creek

$0.204140 \quad 0.183740$

$-0.29819 \quad-0.32391$

$-0.32391$

$-0.39042$

$-0.03062 \quad-0.04473$

$-0.26998$

$-0.16833$

$-0.33732$

$-0.23394$
McBean Tobacco Rd Congaree $-0.26453$

$-0.03062$

$-0.04473$

$-0.48694$

0.007605

0.112597
0.236954

$-0.26998$

$-0.33732$

0.007605

$-0.37718$

$-0.27404$
Pee Dee

0.340096

$-0.46833$

$-0.23394$

0.112597

$-0.27404$

$-0.40516$

Positive values show pairs of means that are significantly different. 
FIGURE 12 .

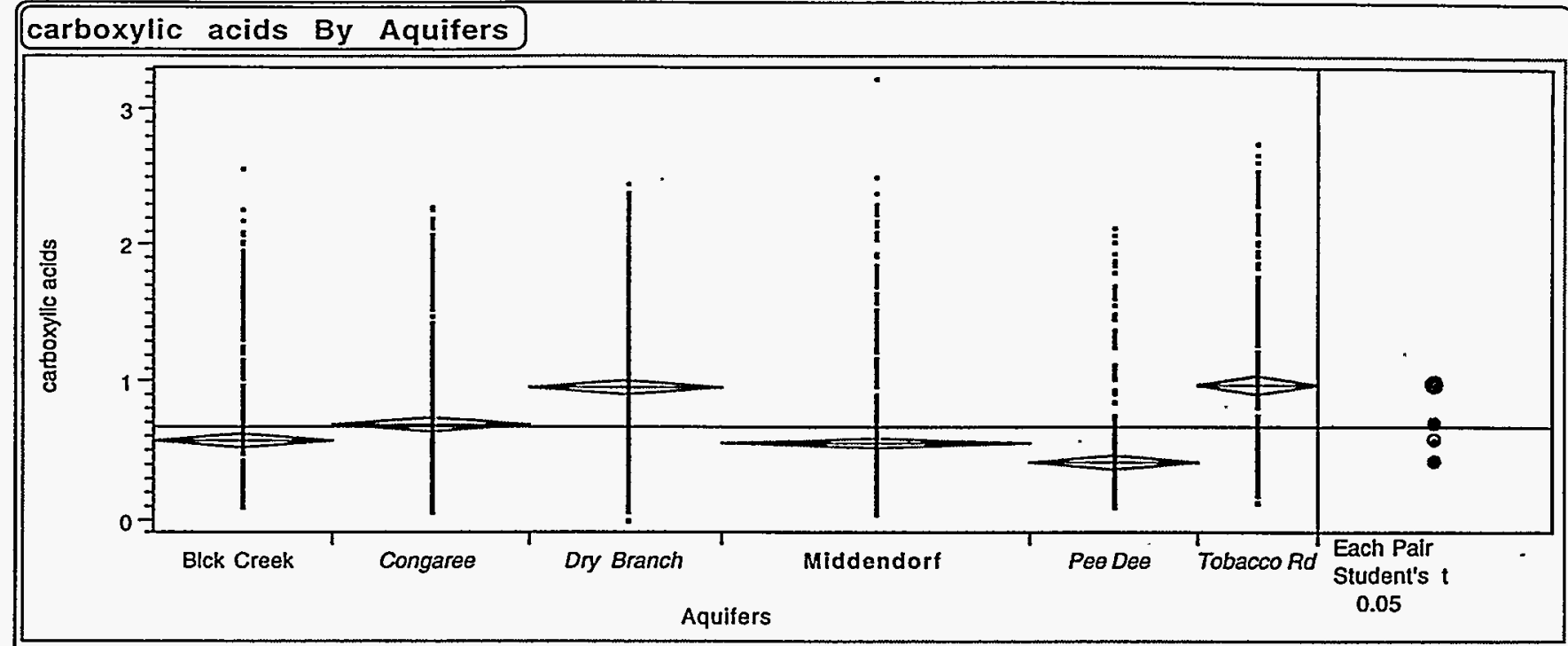

\section{Oneway Anova}

\section{Summary of Fit}

RSquare

0.101771

RSquare Ad]

0.09977

Root Mean Square Error

Mean of Response

0.575794

0.666681

Observations (or Sum Wgts)

2250

\begin{tabular}{|c|c|c|c|c|c|}
\hline \multicolumn{6}{|c|}{ Analysis of Variance } \\
\hline $\begin{array}{l}\text { Source } \\
\text { Model } \\
\text { Error } \\
\text { C Total }\end{array}$ & Sum & $\begin{array}{r}\text { of Squares } \\
84.29378 \\
743.97328 \\
828.26706\end{array}$ & Mean & $\begin{array}{r}\text { Square } \\
16.8588 \\
0.3315\end{array}$ & $\begin{array}{r}\text { F Ratio } \\
50.8500 \\
\text { Prob>F } \\
0.0000\end{array}$ \\
\hline \multicolumn{6}{|c|}{ Means for Oneway Anova } \\
\hline $\begin{array}{l}\text { Level } \\
\text { Blck Creek } \\
\text { Congaree } \\
\text { Dry Branch } \\
\text { McBean } \\
\text { Middendorf } \\
\text { Pee Dee } \\
\text { Tobacco Rd } \\
\text { Std Error uses a po }\end{array}$ & $\begin{array}{r}\text { orer } \\
350 \\
375 \\
375 \\
0 \\
600 \\
325 \\
225 \\
\text { oled }\end{array}$ & $\begin{array}{r}\text { Mean } \\
0.566397 \\
0.688472 \\
0.951947 \\
0.551388 \\
0.417289 \\
0.978596 \\
\text { estimate of en }\end{array}$ & $\begin{array}{r}\text { Std Err } \\
0.030 \\
0.029 \\
0.029 \\
\\
0.023 \\
0.031 \\
0.038 \\
\text { rror varian }\end{array}$ & $\begin{array}{r}\text { ror } \\
78 \\
73 \\
73 \\
\text {. } \\
51 \\
94 \\
39 \\
\text { nce }\end{array}$ & \\
\hline
\end{tabular}

\section{Means Comparisons}

$\begin{array}{lrrr}\text { Dif=Mean[i]-Mean[j] } & \text { Dry Branch } & \text { Congaree } & \text { Blck Creek } \\ \text { Dry Branch } & 0.000000 & 0.263475 & 0.385550 \\ \text { Congaree } & -0.26347 & 0.000000 & 0.122075 \\ \text { Blck Creek } & -0.38555 & -0.12207 & 0.000000 \\ \text { McBean } & \bullet & \bullet & \\ \text { Tobacco Rd } & 0.026649 & 0.290124 & 0.412198 \\ \text { Middendorf } & -0.40056 & -0.13708 & -0.01501 \\ \text { Pee Dee } & -0.53466 & -0.27118 & -0.14911\end{array}$

\begin{tabular}{rrrr} 
McBean & Tobacco Rd & Middendorf & Pee Dee \\
- & -0.02665 & 0.400558 & 0.534657 \\
- & -0.29012 & 0.137084 & 0.271183 \\
- & -0.4122 & 0.015009 & 0.149108 \\
\hline & 0.000000 & 0.427207 & 0.561306 \\
- & -0.42721 & 0.000000 & 0.134099 \\
- & -0.56131 & -0.1341 & 0.000000
\end{tabular}

Alpha $=0.05$

Comparisons for each pair using Student's t

$$
t
$$

1.96106

Abs(DIf)-LSD

Dry Branch

Congaree

Blck Creek

McBean

Tobacco Rd

Middendorf

Pee Dee

Dry Branch

$-0.08246$

0.181012

0.301627

$-0.06857$

0.326228

0.449082

Congaree
0.181012
-0.08246
0.038153
0.194904
0.062753

Blck Creek 0.301627

0.038153

$-0.08536$

0.315712

$-0.06094$

0.185607
McBean Tobacco Rd Middendorf

$0.062753 \quad 0.185607$

$-0.06094 \quad 0.062125$

$0.338936 \quad 0.463379$

$\begin{array}{ll}-0.06519 & 0.056329\end{array}$

$0.056329 \quad-0.08858$

Positive values show pairs of means that are significantly different. 
FIGURE $12 \mathrm{a}$.

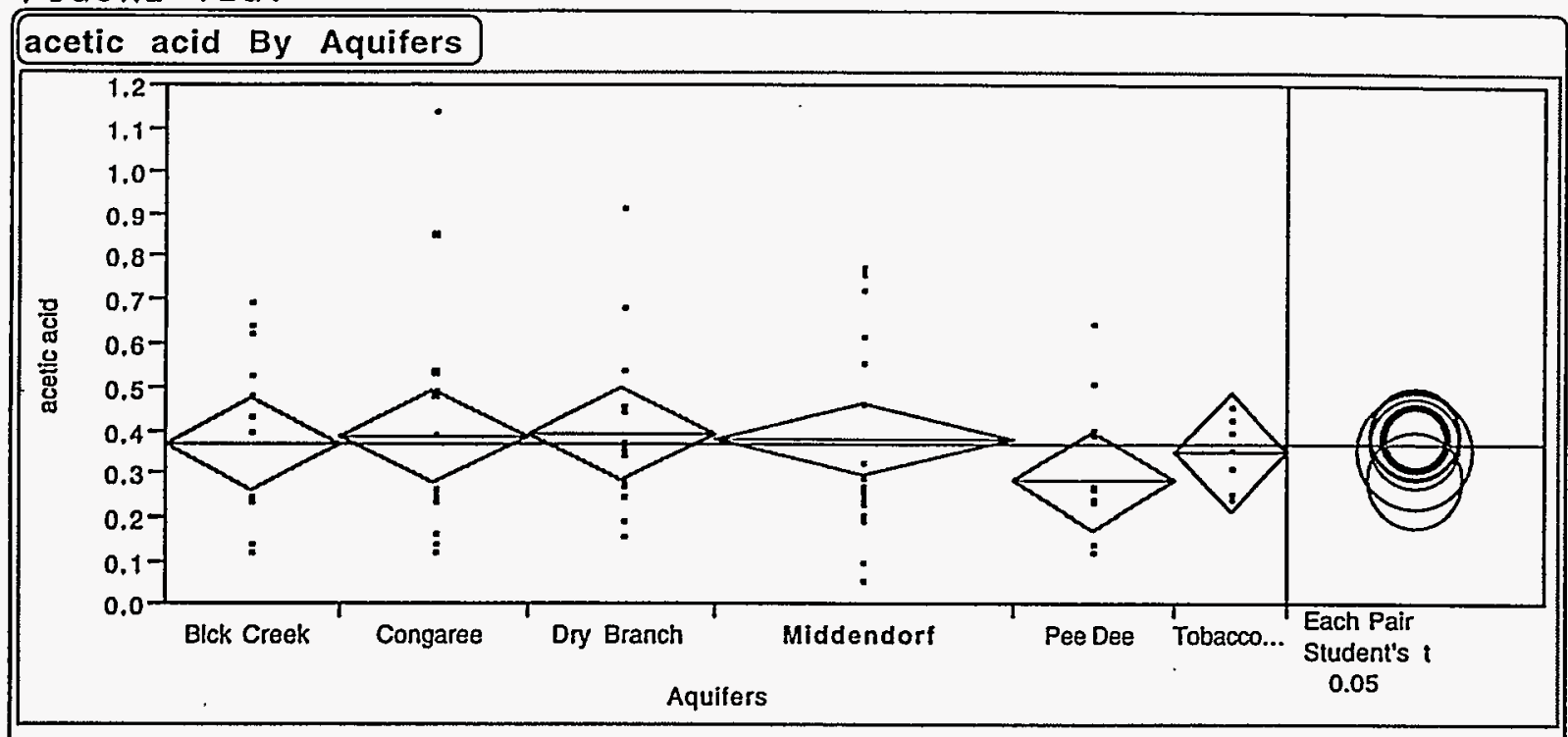

\section{Oneway Anova}

\section{Summary of Fit}

RSquare

RSquare Ad]

Root Mean Square Error

Mean of Response

Observations (or Sum Wgts)

$-0.02971$

0.213367

0.364811

\begin{tabular}{|c|c|c|c|c|c|}
\hline \multicolumn{6}{|c|}{ Analysis of Variance } \\
\hline $\begin{array}{l}\text { Source } \\
\text { Model } \\
\text { Error } \\
\text { C Total }\end{array}$ & $\begin{array}{r}\text { DF } \\
5 \\
84 \\
89\end{array}$ & Sum & $\begin{array}{r}\text { of Squares } \\
0.1107393 \\
3.8241225 \\
3.9348618\end{array}$ & $\begin{array}{r}\text { Mean Square } \\
0.022148 \\
0.045525 \\
\end{array}$ & $\begin{array}{r}\text { F Ratio } \\
0.4865 \\
\text { Prob }>F \\
0.7855\end{array}$ \\
\hline \multicolumn{6}{|c|}{ Means for Oneway Anova } \\
\hline $\begin{array}{l}\text { Level } \\
\text { Blck Creek } \\
\text { Congaree } \\
\text { Dry Branch } \\
\text { McBean } \\
\text { Middendorf } \\
\text { Pee Dee } \\
\text { Tobacco Rd } \\
\text { Std Error use }\end{array}$ & Num & $\begin{array}{r}\text { aber } \\
14 \\
15 \\
15 \\
0 \\
24 \\
13 \\
9 \\
\text { ooled e }\end{array}$ & $\begin{array}{r}\text { Mean S } \\
0.368500 \\
0.386400 \\
0.392200 \\
\text {. } \\
0.381708 \\
0.285000 \\
0.347667 \\
\text { estimate of erro }\end{array}$ & $\begin{array}{r}\text { Std Error } \\
0.05702 \\
0.05509 \\
0.05509 \\
. \\
0.04355 \\
0.05918 \\
0.07112 \\
\text { ror variance }\end{array}$ & \\
\hline
\end{tabular}

\section{Means Comparisons}

\begin{tabular}{lrrrr}
\hline Dif=Mean[i]-Mean[1] & Dry Branch & Congaree & Blck Creek \\
Dry Branch & 0.000000 & 0.005800 & 0.023700 \\
Congaroe & -0.0058 & 0.000000 & 0.017900 \\
Blck Creek & -0.0237 & -0.0179 & 0.000000 \\
McBean & - & & \\
Mlddendorf & -0.01049 & -0.00469 & 0.013208 \\
Tobacco Rd & -0.04453 & -0.03873 & -0.02083 \\
Peo Deo & -0.1072 & -0.1014 & -0.0835
\end{tabular}

$$
\begin{array}{rrrr}
\text { McBean } & \text { Middendorf } & \text { Tobacco Rd } & \text { Pee Dee } \\
* & 0.010492 & 0.044533 & 0.107200 \\
- & 0.004692 & 0.038733 & 0.101400 \\
- & -0.01321 & 0.020833 & 0.083500 \\
- & \bullet & \bullet & \bullet \\
- & 0.000000 & 0.034042 & 0.096708 \\
- & -0.03404 & 0.000000 & 0.062667 \\
& -0.09671 & -0.06267 & 0.000000
\end{array}
$$

Alpha $=0.05$

Comparisons for each pair using Student's $t$ 1

1.98862

Abs(Dif)-LSD

Dry Branch

Congareo

Blck Creek

McBean

Middendorf

Tobacco Rd

Pee Dee

Dry Branch
-0.15493
-0.14913
-0.13398
-
-0.12916
-0.13437
-0.05358

Congaree

$-0.14913$

$-0.15493$

$-0.13978$

$-0.13496$

$-0.14017$

$-0.05938$
BIck Creek $-0.13398$

$-0.13978$

$-0.16037$

$-0.12948$

$-0.16045$

$-0.07993$
McBean Middendorf Tobacco Rd $-0.12916$

$-0.13496$

$-0.12948$

$-0.12249$

$-0.13181$

-0.04941 .
$-0.13437$

$-0.14017$

$-0.16045$

$-0.13181$

$-0.20002$

$-0.12132$
Pee Dee $-0.05358$ $-0.05938$ $-0.07993$

$-0.04941$

$-0.12132$

$-0.16643$

Posilive values show pairs of means that are significantly different. 
FIGURE $12 \mathrm{~b}$.

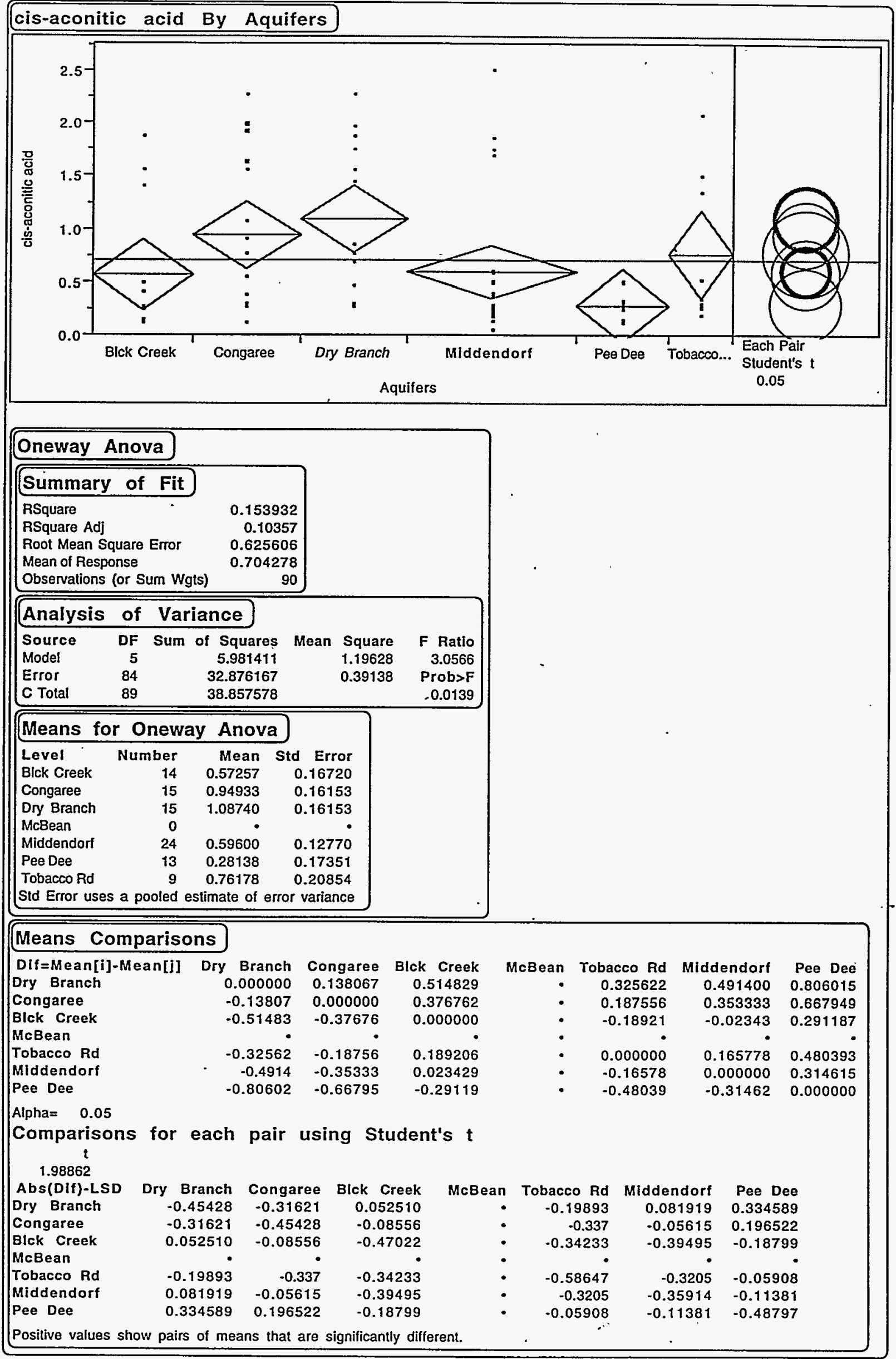


FIGURE $12 \mathrm{c}$.

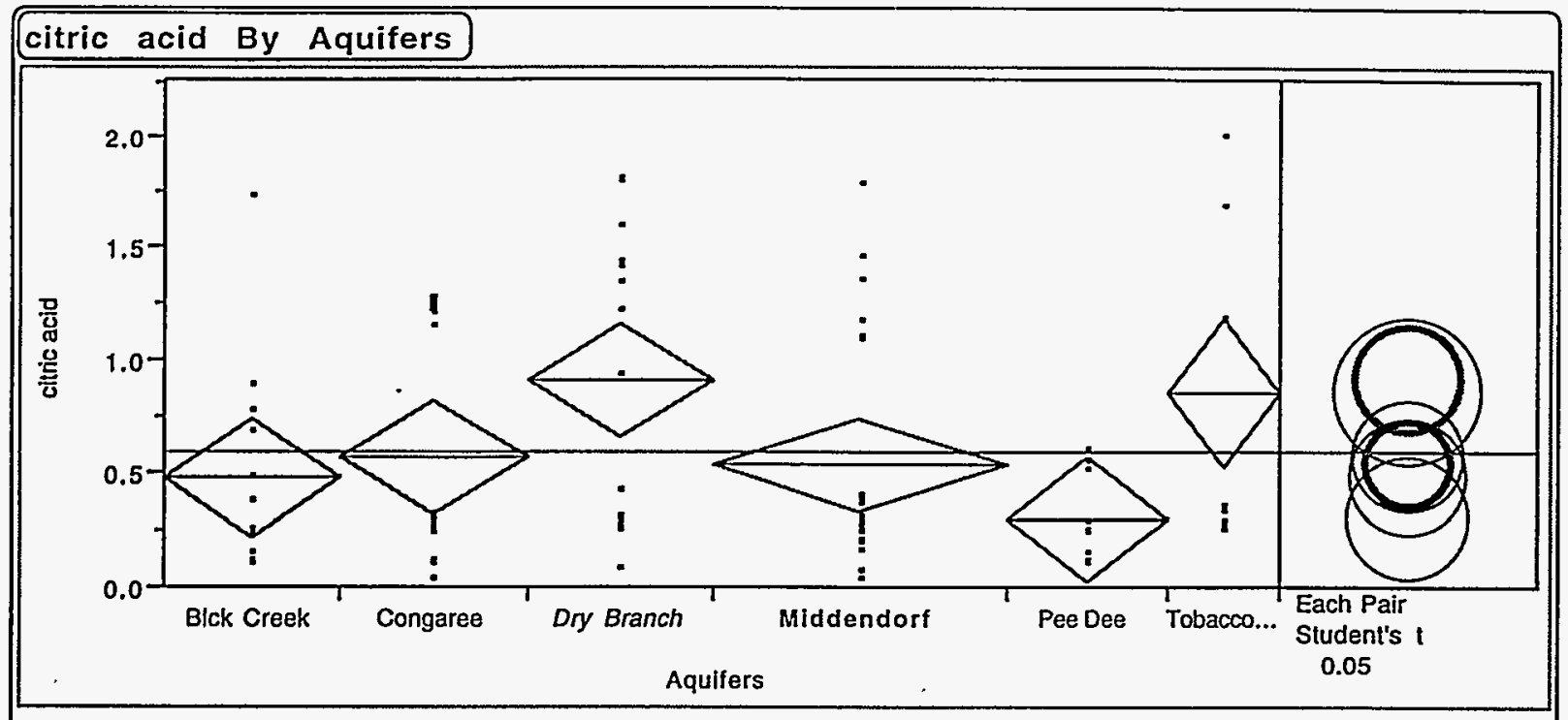

\section{Oneway Anova}

\begin{tabular}{|lr|}
\hline Summary of Fit & \\
RSquare & 0.138878 \\
RSquare Adj & 0.08762 \\
Root Mean Square Error & 0.503472 \\
Mean of Response & 0.590567 \\
Observations (or Sum Wgts) & 90 \\
\hline
\end{tabular}

\begin{tabular}{|lrrrr|}
\hline Analysis & of & Variance & & \\
Source & DF & Sum of Squares & Mean Square & F Ratio \\
Model & 5 & 3.433971 & 0.686794 & 2.7094 \\
Error & 84 & 21.292619 & 0.253484 & Prob $>F$ \\
C Total & 89 & 24.726590 & & 0.0255 \\
\hline
\end{tabular}

Means for Oneway Anova

Lovel Number Mean Std Error

$\begin{array}{llll}\text { Blck Creek } & 14 & 0.480357^{\circ} & 0.13456\end{array}$

$\begin{array}{llll}\text { Congaree } & 15 & 0.566200 & 0.13000\end{array}$

$\begin{array}{llll}\text { Dry Branch } & 15 & 0.905333 & 0.13000\end{array}$

McBean

$\begin{array}{llll}\text { Middendorf } & 24 & 0.532708 & 0.10277\end{array}$

$\begin{array}{llll}\text { Pee Dee } & 13 & 0.301692 & 0.13964 \\ & & 0.849556 & 0.16782\end{array}$

$\begin{array}{llll}\text { Tóbacco Rd } & 9 & 0.849556 & 0.16782\end{array}$

Sid Error uses a pooled estimate of error variance

\section{Means Comparisons}

$\begin{array}{lrrr}\text { Dlf=Mean[I]-Mean[J] } & \text { Dry Branch } & \text { Congaree } & \text { Blck Creek } \\ \text { Dry Branch } & 0.000000 & 0.339133 & 0.424976 \\ \text { Congaree } & -0.33913 & 0.000000 & 0.085843 \\ \text { Blck Creek } & -0.42498 & -0.08584 & 0.000000 \\ \text { McBean } & - & & \\ \text { Tobacco Rd } & -0.05578 & 0.283356 & 0.369198 \\ \text { Mlddendorf } & -0.37262 & -0.03349 & 0.052351 \\ \text { Peo Dee } & -0.60364 & -0.26451 & -0.17866\end{array}$

McBean Tobacco Rd Middendorf

$\begin{array}{ll}0.033492 & 0.264508\end{array}$

$\begin{array}{rr}-0.05235 & 0.178665\end{array}$

0.316847

0.231016

$\begin{array}{ll}-0.23102 & 0.000000\end{array}$

Alpha $=0.05$

Comparisons for each pair using Student's t $t$ 1.98862

Abs(DIt)-LSD

Dry Branch

Congareo

Blck Creek

McBean

Tobacco Rd

Mlddendorf

Par Dee

Dry Branch $-0.36559$

$-0.02646$

0.052914

$-0.36637$

0.043086

0.224249
McBean Tobacco Rd $-0.36637$

$-0.13879$

$-0.05857$

$-0.47198$

$-0.07449$

0.113708

Positive values show pairs of means that are significantly different.

Middendorf Pee Dee $0.043086 \quad 0.224249$

$-0.29605-0.11488$

$-0.28435-0.20697$

$-0.07449$

0.113708

$-0.28903-0.11377$

$-0.11377-0.39271$ 
FIGURE $12 \mathrm{~d}$.

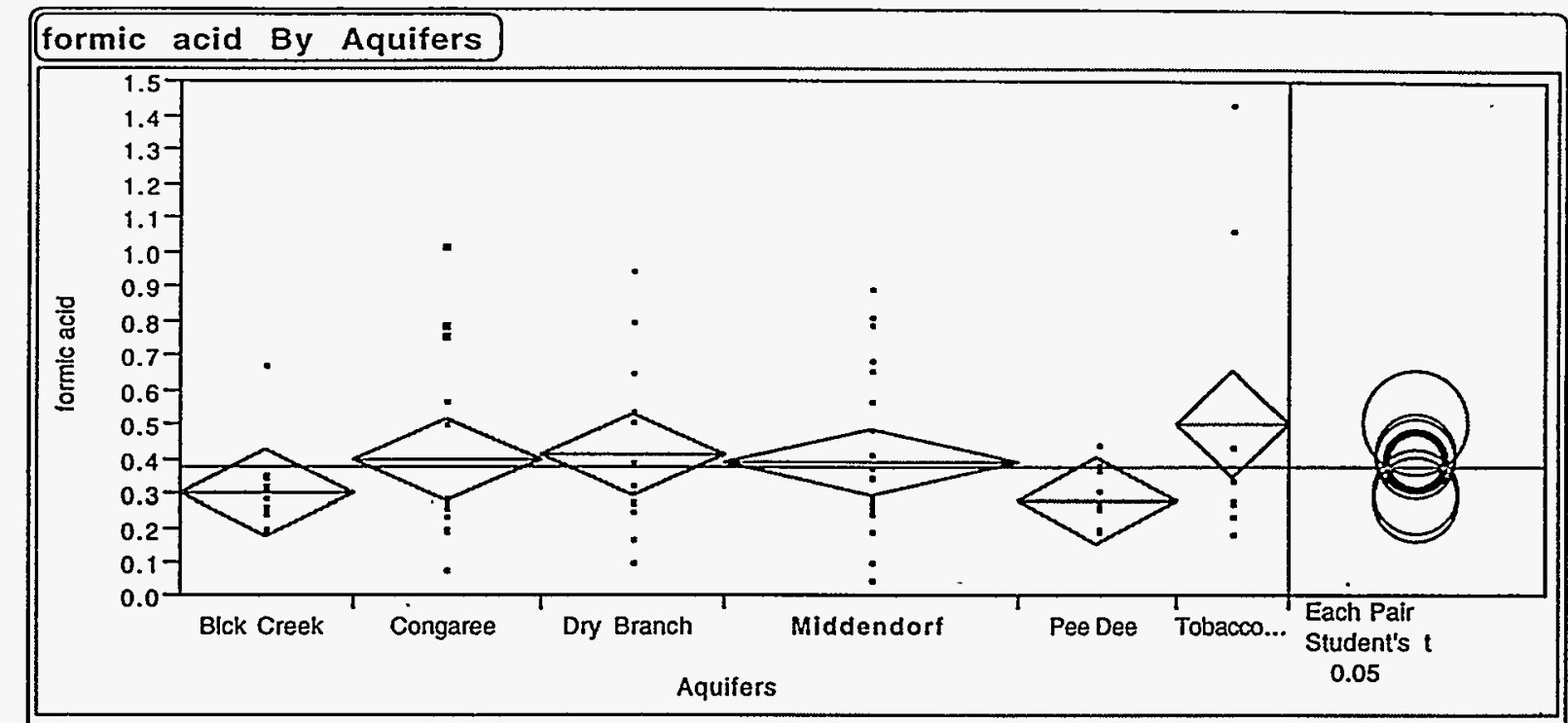

\section{Oneway Anova}

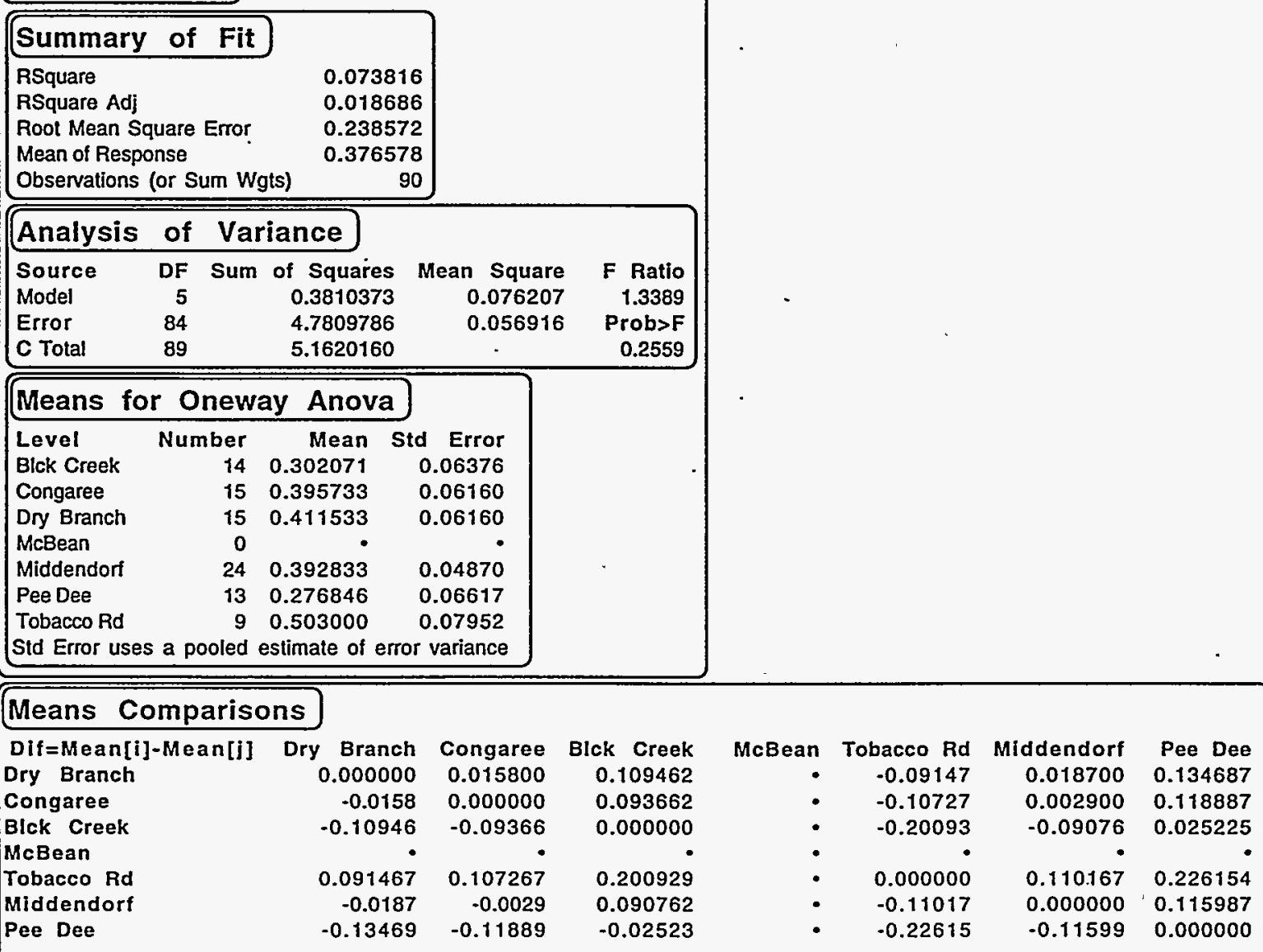

Alpha $=0.05$

Comparisons for each pair using Student's $t$

\section{t}

1.98862

Abs(Dif)-LSD Dry Branch Congaree Blck Creek

Dry Branch

Congaree

Blck Creek

$-0.17324$

$-0.15744$

$-0.15744$

$-0.06684$

McBean

Tobacco Rd

$-0.06684$

$-0.17324$

$-0.08264$

McBean Tobacco Rd Middendorf Pee Dee

Middendor

$-0.08264$

$-0.17932$

$\begin{array}{lll}-0.10857 & -0.09277 & -0.00177\end{array}$

$\begin{array}{lll}-0.13745 & -0.15325 & -0.06879\end{array}$

$\begin{array}{lll}-0.04509 & -0.06089 & -0.15751\end{array}$

Positive values show pairs of means that are significantly different. 
FIGURE $12 \mathrm{e}$.

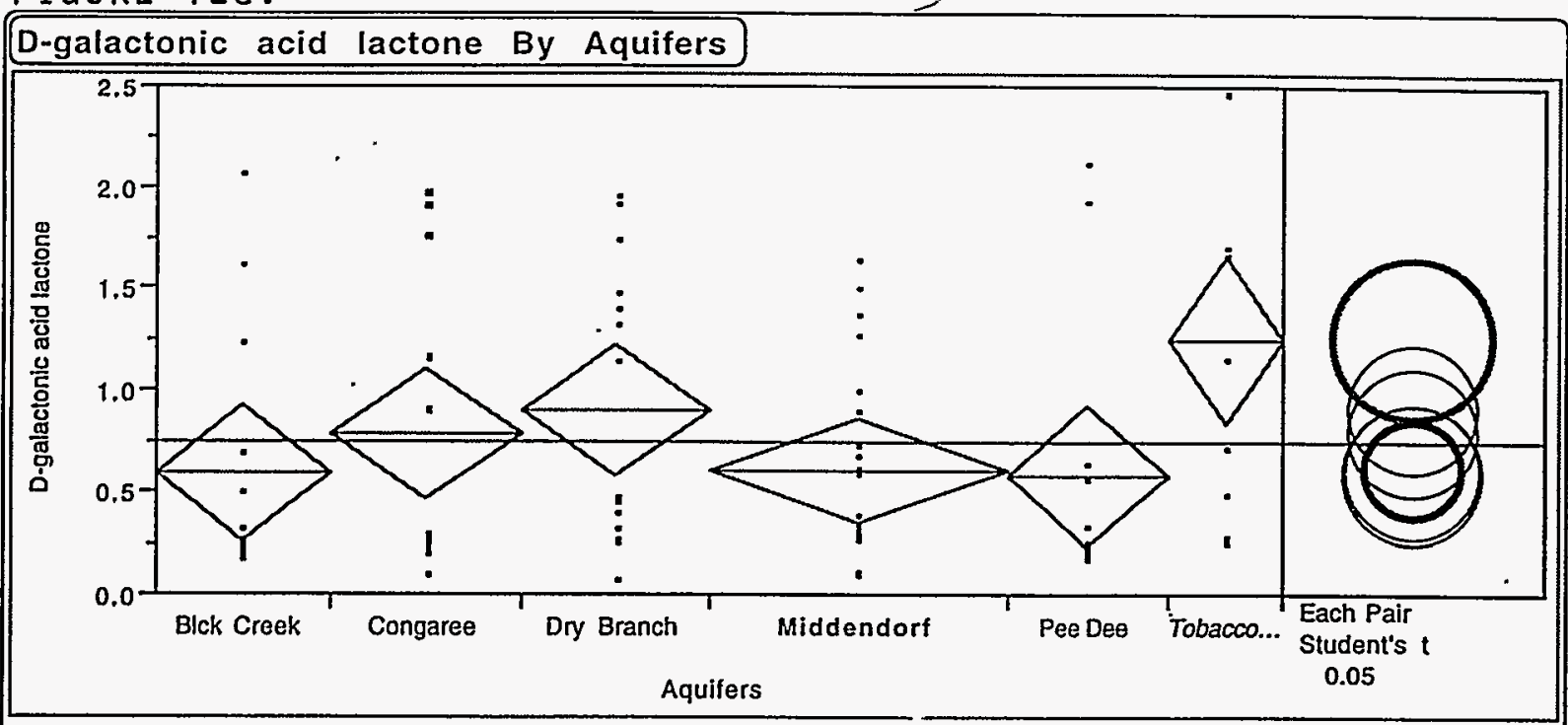

\section{Oneway Anova}

\section{Summary of Fit}

RSquare

0.100042

RSquare Ad

0.046473

Root Mean Square Error

Mean of Response

0.632288

Observations (or Sum Wgts)

0.7445

\section{Analysis of Variance}

\begin{tabular}{lrrrrr} 
Source & DF & Sum of Squares & Mean Square & F Ratio \\
Model & 5 & 3.733083 & 0.746617 & 1.8675 \\
Error & 84 & 33.582178 & 0.399788 & Prob $>F$ \\
C Total & 89 & 37.315260 & & 0.1087 \\
\hline
\end{tabular}

\begin{tabular}{lrrrr}
\hline Means for Oneway & \multicolumn{2}{c}{ Anova } & \\
Level & Number & Mean & Std & Error \\
Blck Creek & 14 & 0.59757 & 0.16899 \\
Congaree & 15 & 0.77980 & 0.16326 \\
Dry Branch & 15 & 0.89933 & 0.16326 \\
McBean & 0 & & \\
Middendort & 24 & 0.61233 & 0.12907 \\
Pee Dee & 13 & 0.57908 & 0.17537 \\
Tobacco Ad & 9 & 1.24756 & 0.21076
\end{tabular}

Std Error uses a pooled estimate of error variance

\section{Means Comparisons}

\begin{tabular}{|c|c|c|c|c|c|c|c|}
\hline DIf=Mean[i]-Mean[j] & Dry Branch & Congaree & Blck Creek & McBean & Tobacco Rd & Middendorf & Pee Dee \\
\hline Dry Branch & 0.000000 & 0.119533 & 0.301762 & - & -0.34822 & 0.287000 & 0.320256 \\
\hline Congareo & -0.11953 & 0.000000 & 0.182229 & - & -0.46776 & 0.167467 & 0.200723 \\
\hline Blck Crook & -0.30176 & -0.18223 & 0.000000 & - & -0.64998 & -0.01476 & 0.018495 \\
\hline cBean & - & - & $\bullet$ & - & $\bullet$ & $\bullet$ & • \\
\hline bbacco Rd & 0.348222 & 0.467756 & 0.649984 & - & 0.000000 & 0.635222 & 0.668479 \\
\hline Middendorf & -0.287 & -0.16747 & 0.014762 & - & -0.63522 & 0.000000 & 0.033256 \\
\hline Pee Dee & -0.32026 & -0.20072 & -0.01849 & - & -0.66848 & -0.03326 & 0.000000 \\
\hline
\end{tabular}

Alpha $=0.05$

Comparisons for each pair using Student's $t$

1.98862

Abs(DIf)-LSD Dry Branch

Dry Branch

Congaree

Blck Croek

McBean

Tobacco Rd

Middendorf

Pee Dee

$-0.45913$

$-0.3396$

$-0.16549$

Congaree $-0.3396$

Blck Creek

$-0.45913$

$-0.16549$

$-0.18194 \quad-0.0624$

$-0.12685$

$-0.15621$

-0.0624
-0.24639

$-0.27574$
$-0.28503$

$-0.47524$

0.112773

$-0.40809$

$-0.4658$
McBean Tobacco Rd Middendorf

Pee Dee

$\begin{array}{rr}-0.24639 & -0.27574\end{array}$

$-0.40809 \quad-0.4658$

$0.112773-0.40809$

0.143753

$-0.36297-0.39975$

$-0.39975-0.49318$

Positive values show pairs of means that are significantly different. 
FIGURE $12 \mathrm{f}$.

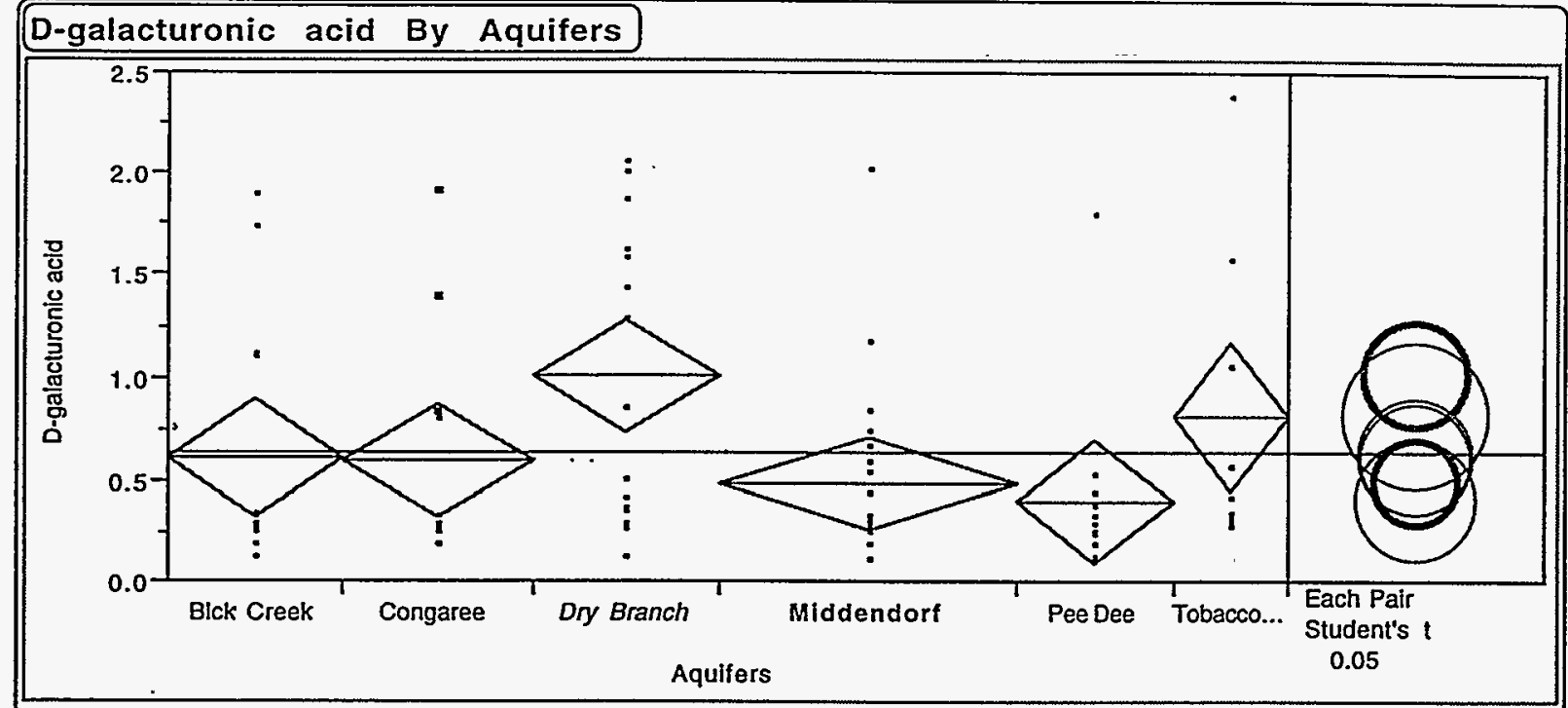

\section{Oneway Anova}

Summary of Fit

RSquare

0.124578

RSquare Adj

0.07247

Root Mean Square Error

Mean of Response

0.556226

0.6274

Observations (or Sum Wgts)

90

\begin{tabular}{|lrrrrr|}
\hline Analysis & of & Variance & & \\
Source & DF & Sum of Squares & Mean Square & F Ratio \\
Model & 5 & 3.698334 & 0.739667 & 2.3907 \\
Error & 84 & 25.988542 & 0.309387 & Prob>F \\
C Total & 89 & 29.686876 & & 0.0445 \\
\hline
\end{tabular}

Means for Oneway Anova

Level Number Mean Std Error

$\begin{array}{llll}\text { Blck Creek } & 14 & 0.60121 & 0.14866\end{array}$

$\begin{array}{llll}\text { Congaree } & 15 & 0.59607 & 0.14362\end{array}$

$\begin{array}{llll}\text { Dry Branch } & 15 & 1.00860 & 0.14362\end{array}$

McBean

Middendorf

Pee Dee

Tobacco Rd

$\begin{array}{llll}9 & 0.80822 & 0.18541\end{array}$

Shan

\section{Means Comparisons}

\begin{tabular}{|c|c|c|c|c|c|c|c|}
\hline Dif $=$ Mean[l]-Mean[j] & Dry Branch & Blck Creek & Congaree & McBean & Tobacco Rd & Middendorf & Pee Dee \\
\hline Dry Branch & 0.000000 & 0.407386 & 0.412533 & 8 & 0.200378 & 0.528725 & 0.609523 \\
\hline Blck Creek & -0.40739 & 0.000000 & 0.005148 & $\bullet$ & -0.20701 & 0.121339 & 0.202137 \\
\hline Congaree & -0.41253 & -0.00515 & 0.000000 & 8 & -0.21216 & 0.116192 & 0.196990 \\
\hline McBean & - & - & - & - & • & $\bullet$ & $\bullet$ \\
\hline Tobacco Rd & -0.20038 & 0.207008 & 0.212156 & - & 0.000000 & 0.328347 & 0.409145 \\
\hline Middendorf & -0.52872 & -0.12134 & -0.11619 & - & -0.32835 & 0.000000 & 0.080798 \\
\hline Pee Dee & -0.60952 & -0.20214 & -0.19699 & - & -0.40915 & -0.0808 & 0.000000 \\
\hline
\end{tabular}

Pee Dee

$-0.20214$

$-0.19699$

$-0.0808$

Comparisons for each pair using Student's, $t$

\begin{tabular}{lrrrr}
\multicolumn{1}{c}{$t$} & & & \\
\multicolumn{1}{l}{ 1.98862 } & & & \\
Abs(Dif)-LSD & Dry & Branch & Blck Creek & Congaree \\
Dry Branch & -0.4039 & -0.00366 & 0.008635 \\
Blck Creek & -0.00366 & -0.41807 & -0.4059 \\
Congaree & 0.008635 & -0.4059 & -0.4039 \\
McBean & - & $\bullet$ &. \\
Tobacco Rd & -0.266 & -0.26558 & -0.25423 \\
Middendorf & 0.164656 & -0.25065 & -0.24788 \\
Pee Dee & 0.190378 & -0.2239 & -0.22216
\end{tabular}

Positive values show pairs of means that are significantly different.

\begin{tabular}{|c|c|c|c|}
\hline McBean & Tobacco Rd & Middendorf & Pee Dee \\
\hline$\bullet$ & -0.266 & 0.164656 & 0.190378 \\
\hline - & -0.26558 & -0.25065 & -0.2239 \\
\hline - & -0.25423 & -0.24788 & -0.22216 \\
\hline - & - & - & - \\
\hline - & -0.52143 & -0.104 & -0.0705 \\
\hline - & -0.104 & -0.31931 & -0.30012 \\
\hline - & -0.0705 & -0.30012 & -0.43386 \\
\hline
\end{tabular}


FIGURE $12 \mathrm{~g}$.

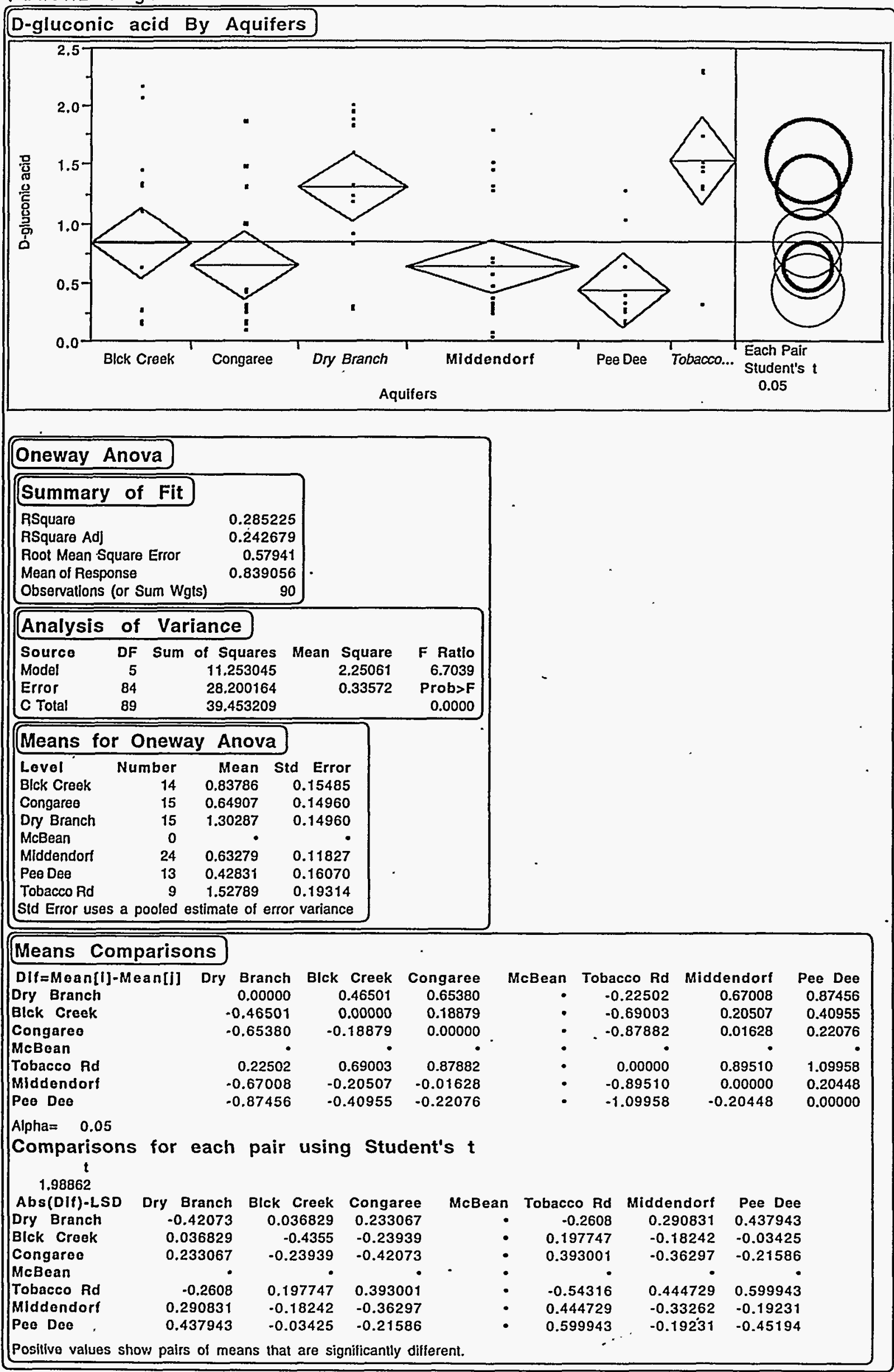




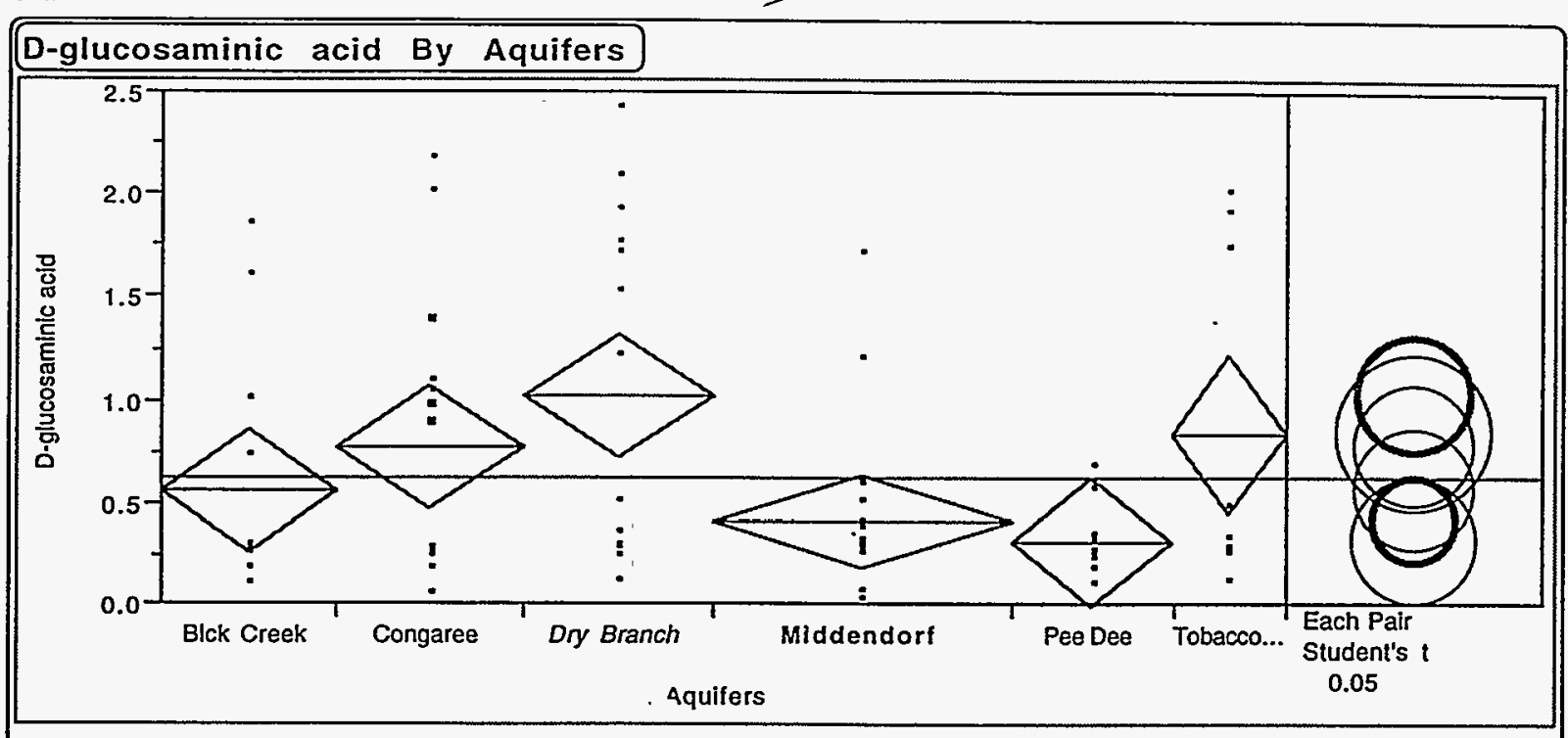

\section{Oneway Anova}

\begin{tabular}{|lr|}
\hline Summary of Fit & \\
RSquare & 0.164126 \\
RSquare Adj & 0.114372 \\
Root Mean Square Error & 0.584924 \\
Mean of Response & 0.619544 \\
Observations (or Sum Wgts) & 90 \\
\hline
\end{tabular}

\begin{tabular}{|lrrrrr|}
\hline \hline Analysis & of & Variance & & \\
Source & DF & Sum of Squares & Mean Square & F Ratio \\
Model & 5 & 5.643069 & 1.12861 & 3.2987 \\
Error & 84 & 28.739463 & 0.34214 & Prob $>$ F \\
C Total & 89 & 34.382532 & & 0.0091 \\
\hline
\end{tabular}

Means for Oneway Anova

Level Number Mean Std Error

$\begin{array}{llll}\text { Blck Creek } & 14 & 0.55586 & 0.15633\end{array}$

$\begin{array}{llll}\text { Congaree } & 15 & 0.76513 & 0.15103\end{array}$

$\begin{array}{llll}\text { Dry Branch } & 15 & 1.02233 & 0.15103\end{array}$

McBean

$\begin{array}{lrrr}\text { Middendorf } & 24 & 0.40133 & 0.11940 \\ \text { Pee Dee } & 13 & 0.30754 & 0.16223\end{array}$

$\begin{array}{lrll}\text { Tobacco Rd } & 9 & 0.83722 & 0.19497\end{array}$

Std Error uses a pooled estimate of error variance

\section{Means Comparisons}

Dif=Mean[i]-Mean[j] Dry Branc Dry Branch

Congaree

Blck Creek

McBean

Tobacco Ro

Middendorf

Pee Dee

Alpha $=0.05$

Comparisons for each pair using Student's t
Congaree Bick Creek

$0.257200 \quad 0.466476$

$\begin{array}{lll}-0.2572 & 0.000000 & 0.209276\end{array}$

$\begin{array}{lll}-0.46648 & -0.20928 & 0.000000\end{array}$

$\begin{array}{lll}-0.621 & -0.3638 & -0.15452\end{array}$

$\begin{array}{lll}-0.71479 & -0.45759 & -0.24832\end{array}$ $\begin{array}{lll}-0.18511 & 0.072089 & 0.281365\end{array}$
McBean Tobacco Rd Middendorf

0.185111

$-0.07209$

$-0.28137$

0.000000

$-0.43589$

$-0.52968$
0.621000

0.363800

0.154524

0.435889

0.000000

$-0.09379$
Pee Dee 0.714795 0.457595 0.248319

0.529684 0.093795 0.000000
1.98862

Abs(DIf)-LSD Dry Branch Congaree Blck Creek

Dry Branch

Congaree

8lck Creek

McBean

Tobacco Rd

Middendorf

Pee Dee

$$
\begin{array}{rrr}
-0.42474 & -0.16754 & 0.034221 \\
-0.16754 & -0.42474 & -0.22298 \\
0.034221 & -0.22298 & -0.43964 \\
-0.30533 & -0.41836 & -0.2156 \\
0.238147 & -0.01905 & -0.23665
\end{array}
$$$$
0.274024 \quad 0.016824
$$

$-0.1997$
McBean Tobacco Rd Middendorf

$-0.01905 \quad 0.016824$

$-0.23665 \quad-0.1997$

$\begin{array}{rr}-0.01877 & 0.025290\end{array}$

$\begin{array}{ll}-0.33578 & -0.30677\end{array}$

$-0.30677 \quad-0.45624$

Positive values show pairs of means that are signilicantly different. 
FIGURE $12 i$.

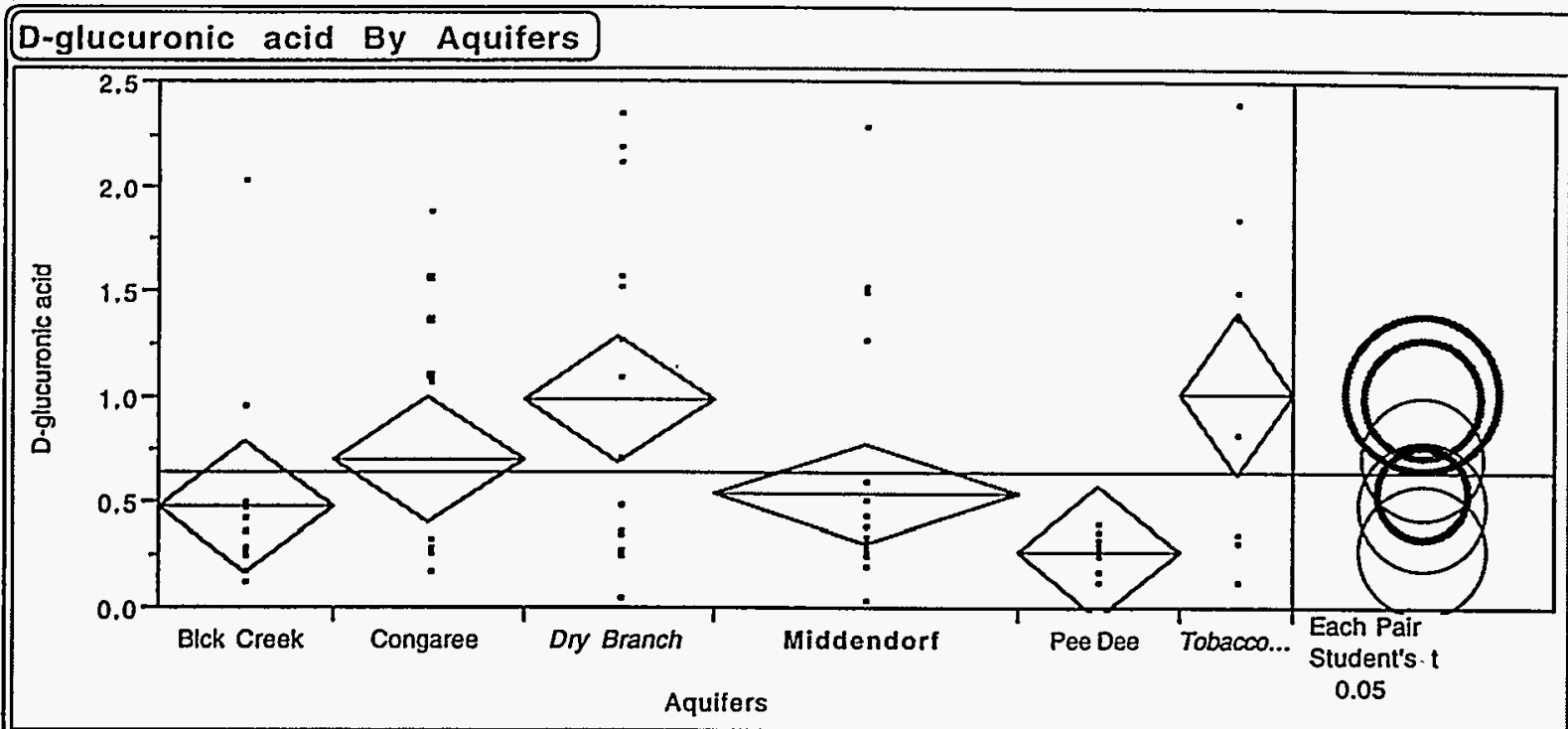

\section{Oneway Anova}

Summary of Fit

RSquare

0.164445

0.11471

RSquare Adj

0.58576

Root Mean Square Error

Mean of Response

0.638211

Observations (or Sum Wgts)

90

\begin{tabular}{|lrrrrr|}
\hline Analysis & of & Variance & & & \\
Source & DF & Sum of Squares & Mean Square & F Ratio \\
Model & 5 & 5.672364 & 1.13447 & 3.3064 \\
Error & 84 & 28.821635 & 0.34311 & Prob>F \\
C Total & 89 & 34.493999 & & 0.0089 \\
\hline
\end{tabular}

Means for Oneway Anova

Lovel Number Mean Std Error

$\begin{array}{llll}\text { Blck Creek } & 14 & 0.47429 & 0.15655\end{array}$

$\begin{array}{llll}\text { Congaree } & 15 & 0.69880 & 0.15124\end{array}$

$\begin{array}{llll}\text { Dry Branch } & 15 & 0.99533 & 0.15124\end{array}$

McBean

Middendorf

Pee Dee

$24 \quad 0.53738$

0.11957

Tobacco Rd

0.26177

0.16246

Sid Error uses a pooled estimat

\section{Means Comparisons}

$\begin{array}{lrrr}\text { Dif=Mean[l]-Mean[]] } & \text { Dry Branch } & \text { Congaree } & \text { Blck Creek } \\ \text { Dry Branch } & 0.000000 & 0.296533 & 0.521048 \\ \text { Congaree } & -0.29653 & 0.000000 & 0.224514 \\ \text { Blck Creek } & -0.52105 & -0.22451 & 0.000000 \\ \text { McBean } & - & - & \\ \text { Tobacco Rd } & 0.014333 & 0.310867 & 0.535381 \\ \text { Middendorf } & -0.45796 & -0.16143 & 0.063089 \\ \text { Pee Dee } & -0.73356 & -0.43703 & -0.21252\end{array}$

Alpha $=0.05$

Comparisons for each pair using Student's $t$
1.98862

Abs(DIf)-LSD

Dry Branch

Congaree

Bick Creek

McBean

Tobacco Rd

Middendorf

Peo Dee

$\begin{array}{rrr}\text { Dry Branch } & \text { Congaree } & \text { Blck Creek } \\ -0.42534 & -0.12881 & 0.088175 \\ -0.12881 & -0.42534 & -0.20836 \\ 0.088175 & -0.20836 & -0.44027 \\ - & \bullet & . \\ -0.47681 & -0.18028 & 0.037702 \\ 0.074558 & -0.22197 & -0.32865 \\ 0.292164 & -0.00437 & -0.23614\end{array}$

McBean Tobacco Rd Middendorf

$-0.22197-0.00437$

$-0.32865$

0.016988

$-0.33626$

$-0.12553$

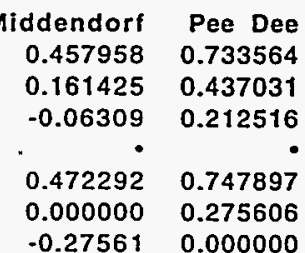

Positive values show pairs of means that are significantly different. 
FIGURE $12 j$.

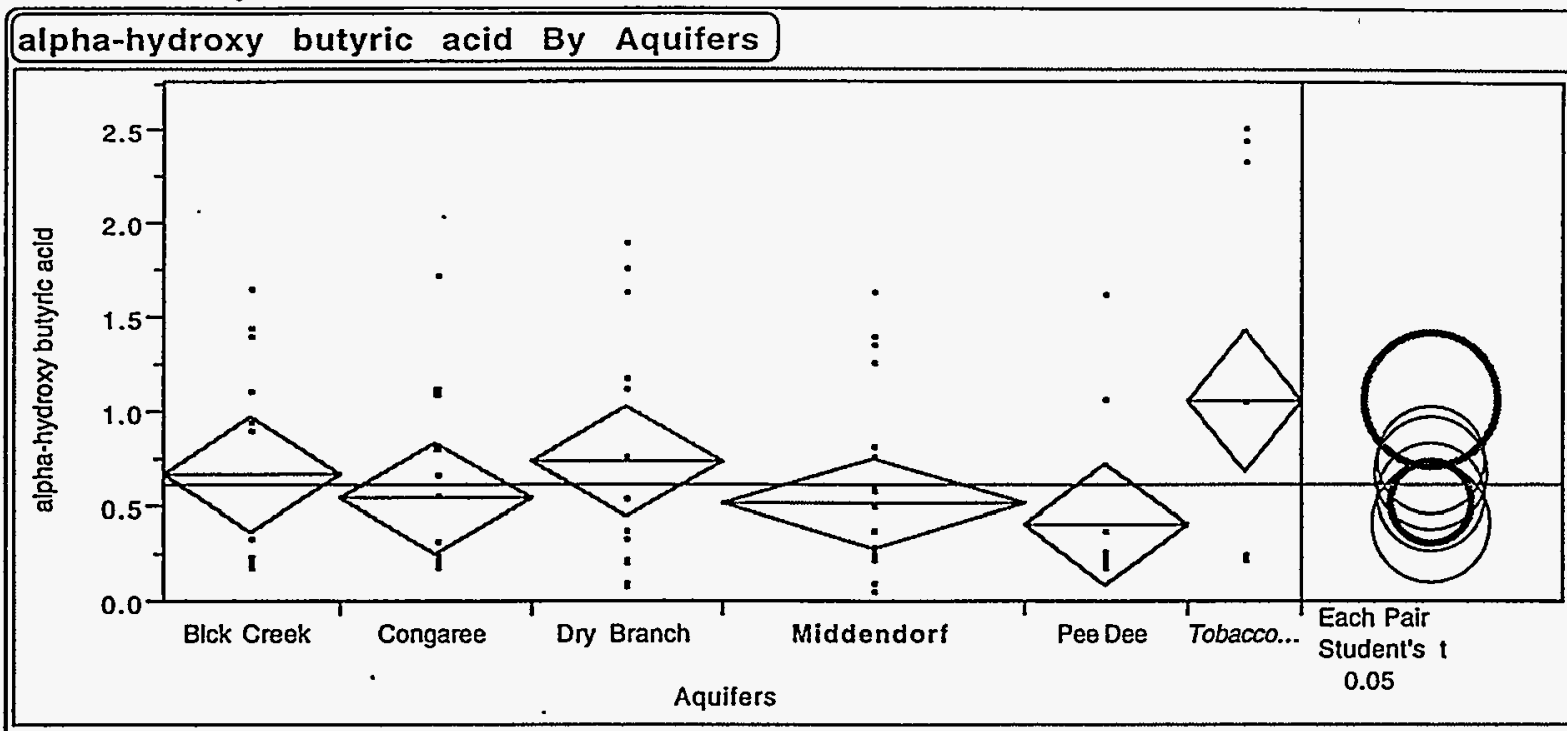

\section{Oneway Anova}

\begin{tabular}{|lr}
\hline Summary of Fit & \\
RSquare & 0.090808 \\
RSquare Adj & 0.03669 \\
Root Mean Square Error & 0.580686 \\
Mean of Response & 0.622144 \\
Observations (or Sum Wgts) & 90 \\
\hline
\end{tabular}

\begin{tabular}{|lrrrrr|}
\hline Analysis & of & \multicolumn{2}{c|}{ Variance } & & \\
Source & DF & Sum of Squares & Mean Square & F Ratio \\
Model & 5 & 2.828989 & 0.565798 & 1.6779 \\
Error & 84 & 28.324486 & 0.337196 & Prob>F \\
C Total & 89 & 31.153475 & & 0.1488 \\
\hline
\end{tabular}

Means for Oneway Anova

Level Number Mean Std Error

$\begin{array}{llll}\text { Blck Creek } & 14 & 0.66757 & 0.15519\end{array}$

$\begin{array}{llll}\text { Congaree * } & 15 & 0.54520 & 0.14993\end{array}$

$\begin{array}{llll}\text { Dry Branch } & 15 & 0.73467 & 0.14993\end{array}$

McBean

Middendorf

Pee Dee

$\begin{array}{rr}0 & \bullet \\ 24 & 0.52383\end{array}$

0.41162

$9 \quad 1.05844$

0.11853

0.16105

0.19356

Std Error uses a pooled estimate of error variance

\section{Means Comparisons}

Dry Branch

Blck Creek

Congaree

McBean

Tobacco Rd

Middendorf

Pee Dee

Alpha $=0.05$

Comparisons for each pair using Student's t

$$
t
$$

1.98862

Abs(Dif)-LSD Dry Branch Blck Creek Congare

Dry Branch

Blck Creek

Congaree

McBean

Tobacco Rd

Middendorf

Pee Dee

$-0.42166$

$-0.36203$

$-0.23219$

$-0.36203$

$-0.43646$

$-0.30675$

$-0.23219$

$-0.30675$

$-0.42166$

$-0.16311$

$-0.16925$

$-0.11453$

$-0.1025$

0.026354

$-0.2446-0.35871$

$-0.18882-0.30399$
McBean Tobacco Rd Middendorf

$-0.16311$

$-0.1025$

0.026354

$-0.54436$

0.083251

0.146090
$-0.16925-0.11453$

$-0.2446-0.18882$

$-0.35871-0.30399$

$0.083251 \quad 0.146090$

$-0.33335-0.28545$

$-0.28545-0.45294$

Positive values show pairs of means that are significantly different. 


\section{F.IGURE' $12 \mathrm{k}$.}

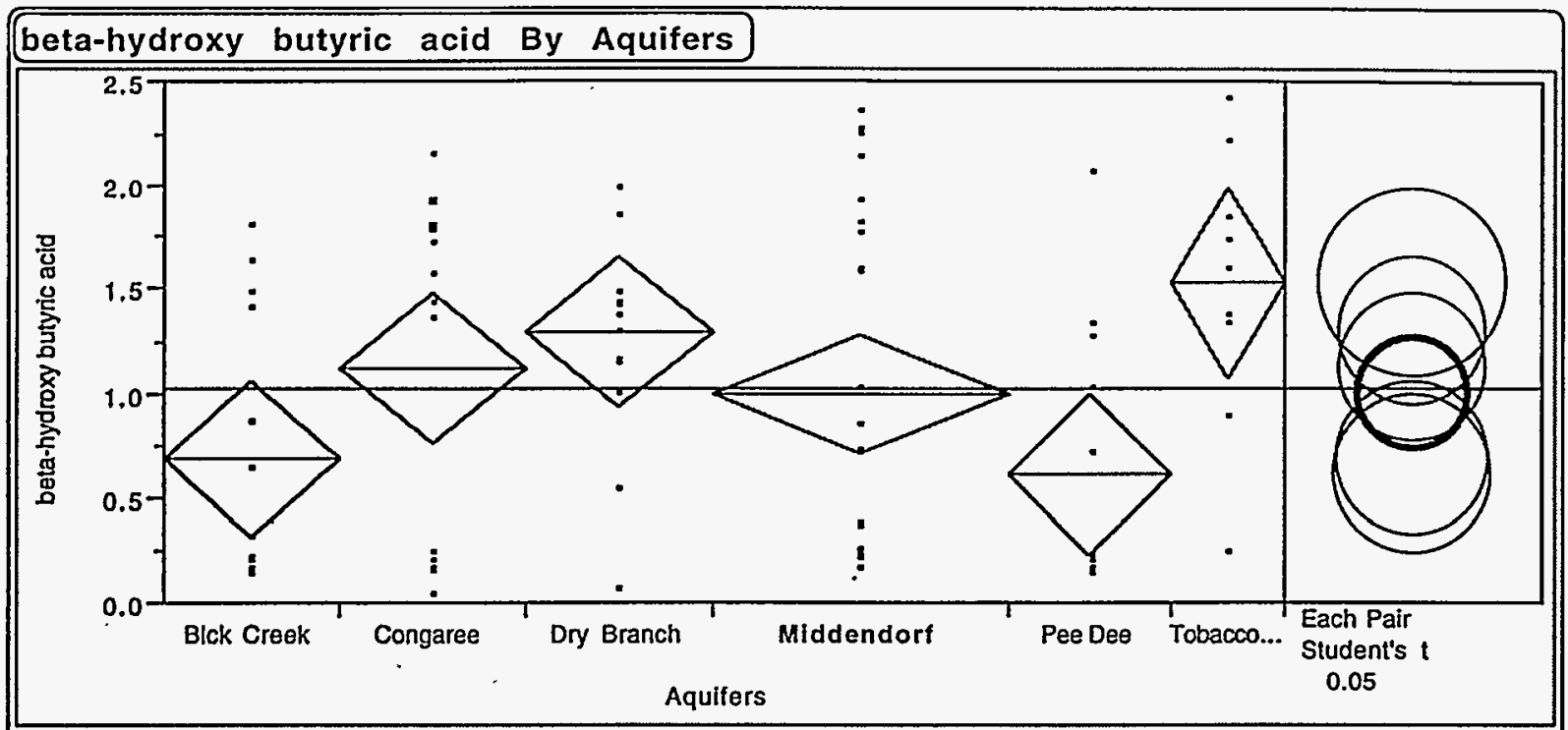

\section{Oneway Anova}

\begin{tabular}{|lr|}
\hline Summary of Fit & \\
RSquare & 0.148348 \\
RSquare Ad] & 0.097654 \\
Root Mean Square Error & 0.704485 \\
Mean of Response & 1.016978 \\
Observations (or Sum Wgts) & 90 \\
\hline
\end{tabular}

\begin{tabular}{||rrrrr|}
\hline Analysis & of & Variance & & \\
Source & DF . Sum of Squares & Mean Square & F Ratio \\
Model & 5 & 7.261756 & 1.45235 & 2.9264 \\
Error & 84 & 41.689072 & 0.49630 & Prob>F \\
C Tolal & 89 & 48.950828 & & .0 .0175 \\
\hline
\end{tabular}

\begin{tabular}{|c|c|c|c|}
\hline \multicolumn{4}{|c|}{ Means for Oneway Anova } \\
\hline $\begin{array}{l}\text { Level } \\
\text { Blck Creek }\end{array}$ & $\begin{array}{r}\text { Number } \\
14\end{array}$ & $\begin{array}{r}\text { Mean } \\
0.68529\end{array}$ & $\begin{array}{r}\text { Std Error } \\
0.18828\end{array}$ \\
\hline Congaree & 15 & 1.12153 & 0.18190 \\
\hline Dry Branch & 15 & 1.29087 & 0.18190 \\
\hline McBean & 0 & - & - \\
\hline Midderidorf & 24 & 0.99925 & 0.14380 \\
\hline Pee Dee & 13 & 0.61692 & 0.19539 \\
\hline Tobacco Rd & 9 & 1.52733 & 0.23483 \\
\hline Std Error us & led & itimate of & \\
\hline
\end{tabular}

\section{Means Comparisons}

Dif=Mean[i]-Mean[i] Dry Branch Dry Branch Congaree Blck Creek McBean

Tobacco Rd Mlddendorf Pee Dee 0.000000 $-0.16933$ $-0.60558$ Congaree 0.169333 0.000000 $-0.43625$

$0.236467 \quad 0.405800$

$\begin{array}{ll}-0.29162 & -0.12228\end{array}$

$-0.67394$
Blck Creek 0.605581

0.436248

0.000000

0.842048

0.313964

$-0.06836$
McBean Tobacco Rd Middendorf

$-0.23647$

-0.23647
-0.4058

$-0.84205$

0.000000

$-0.52808$

$-0.91041$
0.291617
0.122283

$-0.31396$

0.673944

0.504610

0.068363

0.5280830 .910410

$0.000000 \quad 0.382327$

$\begin{array}{ll}-0.38233 & 0.000000\end{array}$

Alpha $=0.05$

Comparisons for each pair using Student's $t$

$$
t
$$

1.98862

Abs(DIf)-LSD

Dry Branch

Congaree

Blck Creek

McBean

Tobacco Rd

Middendorf

Pee Dee

$\begin{array}{rrr}\text { Dry Branch } & \text { Congaree } & \text { Blck Creek } \\ -0.51155 & -0.34222 & 0.084971 \\ -0.34222 & -0.51155 & -0.08436 \\ 0.084971 & -0.08436 & -0.52951 \\ -0.35423 & -0.18489 & 0.243496 \\ -0.16949 & -0.33883 & -0.15717 \\ 0.143078 & -0.02626 & -0.47123\end{array}$

McBean Tobacco Rd

\begin{tabular}{rr}
-0.35423 \\
-0.18489 \\
$: \quad 0.243496$ \\
\hline & -0.66041 \\
- $\quad 0.0195$ \\
\hline & 0.302917
\end{tabular}

Middendorf $-0.16949$

$-0.33883$

$-0.15717$

$-0.0195$

$-0.40442$

$-0.10012$
Pee Dee 0.143078 $-0.02626$

$-0.47123$

0.302917

$-0.10012$

$-0.5495$

Posilive values show pairs of means that are significantly different. 


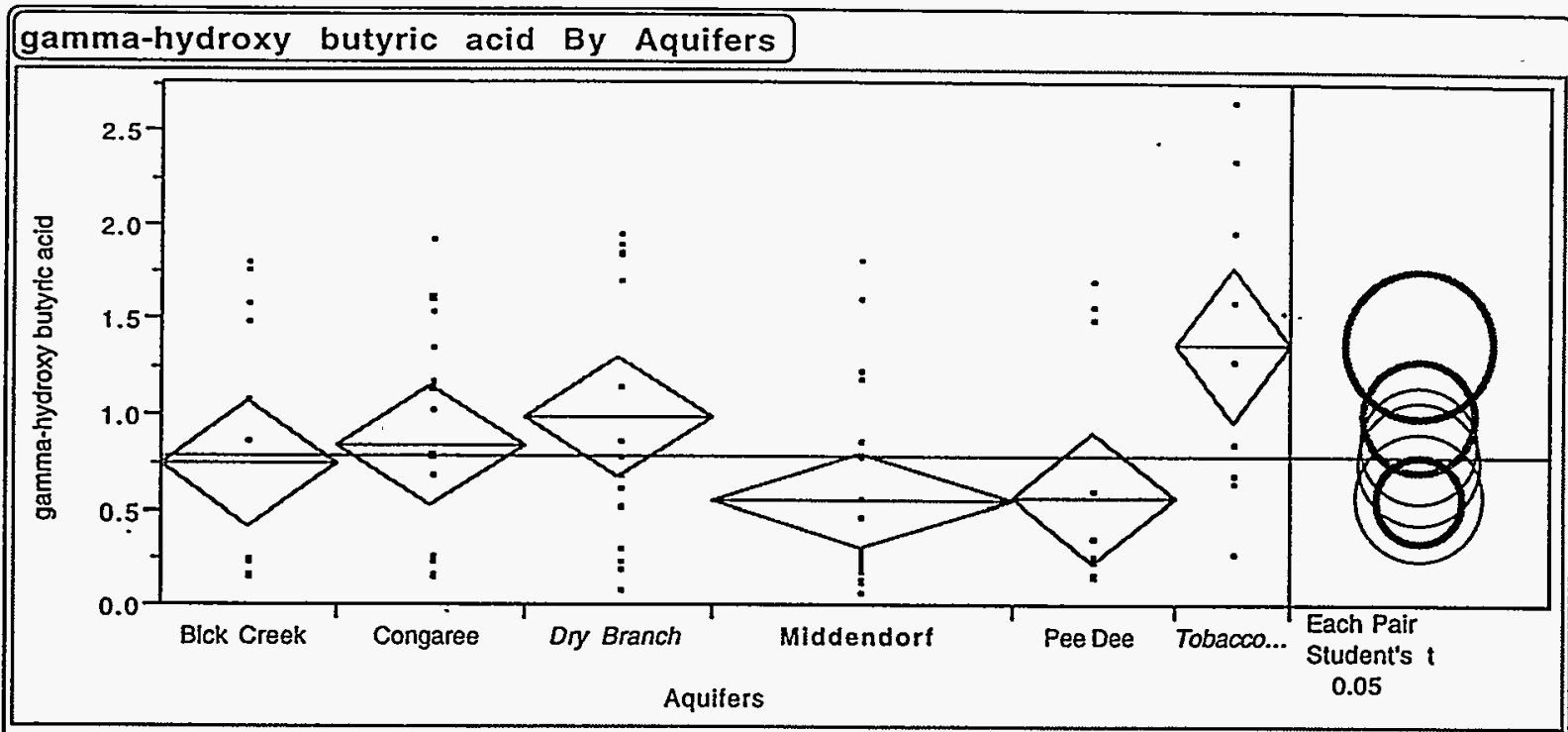

\section{Oneway Anova}

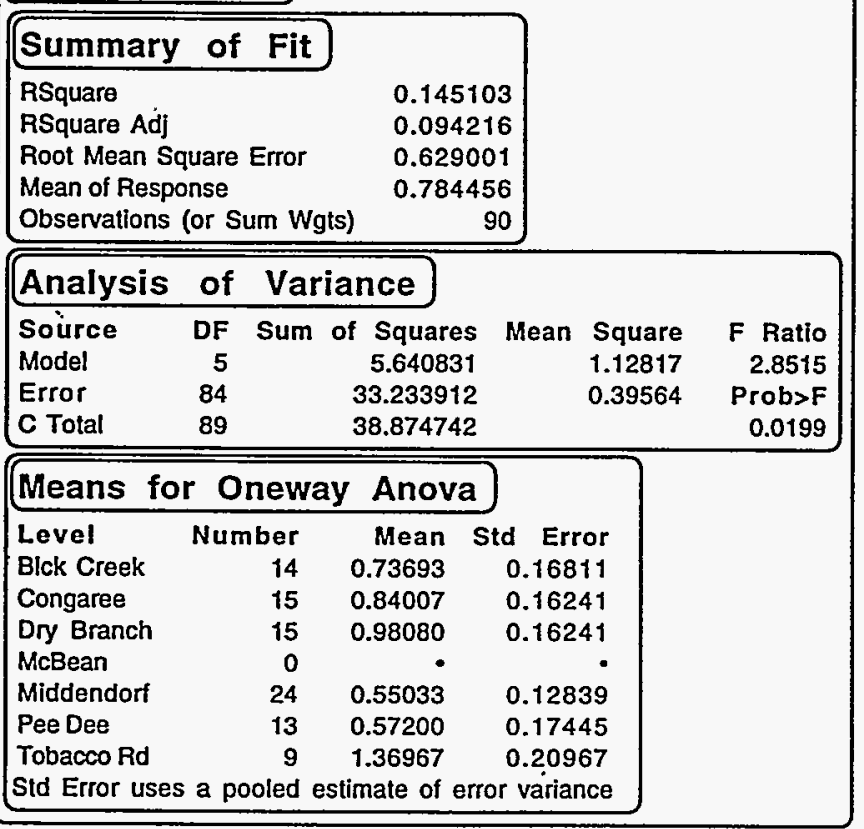

\section{Means Comparisons}

\begin{tabular}{|c|c|c|c|c|c|c|c|}
\hline $\begin{array}{l}\text { Dif=Mean }[i] \text {-Mean }[j] \\
\text { Dry Branch }\end{array}$ & $\begin{array}{r}\text { Dry Branch } \\
0.000000\end{array}$ & $\begin{array}{r}\text { Congaree } \\
0.140733\end{array}$ & $\begin{array}{r}\text { Blck Creek } \\
0.243871\end{array}$ & McBean & $\begin{array}{r}\text { Tobacco Rd } \\
-0.38887\end{array}$ & $\begin{array}{l}\text { Pee Dee } \\
0.408800\end{array}$ & $\begin{array}{r}\text { Middendorf } \\
0.430467\end{array}$ \\
\hline Congaree & -0.14073 & 0.000000 & 0.103138 & - & -0.5296 & 0.268067 & 0.289733 \\
\hline Blck Creek & -0.24387 & -0.10314 & 0.000000 & - & -0.63274 & 0.164929 & 0.186595 \\
\hline McBean & - & $\bullet$ & - & • & - & - & . \\
\hline Tobacco Rd & 0.388867 & 0.529600 & 0.632738 & - & 0.000000 & 0.797667 & 0.819333 \\
\hline Pee Dee & -0.4088 & -0.26807 & -0.16493 & - & -0.79767 & 0.000000 & 0.021667 \\
\hline Middendorf & -0.43047 & -0.28973 & -0.1866 & - & -0.81933 & -0.02167 & 0.000000 \\
\hline
\end{tabular}

Alpha $=0.05$

Comparisons for each pair using Student's $t$

1.98862

Abs(Dif)-LSD

Dry Branch

Congaree

Blck Creek

McBean

Tobacco Rd

Pee Dee

MIddendorf
Dry Branch

$-0.45674$

$-0.31601$

$-0.22096$

$-0.13853$

$-0.06518$

0.018764
Congaree

$-0.31601$

$-0.45674$

$-0.36169$

0.002199

$-0.20592$

$-0.12197$
Blck Creek

$-0.22096$

$-0.36169$

$-0.47277$

0.098320

$-0.31685$

$-0.23406$
McBean Tobacco Rd

- $\quad 0.13853$

- $\quad 0.002199$

- $\quad 0.098320$

- $\quad 0.0$

- 0.58965

- $\quad 0.255265$

- $\quad 0.330419$.
Pee Dee

$-0.06518$

$-0.20592$

$-0.31685$

0.255265

$-0.49062$

$-0.40908$
Middendorf 0.018764

$-0.12197$

$-0.23406$

0.330419

$-0.40908$

$-0.36109$

Positive values show pairs of means that are significantly different. 


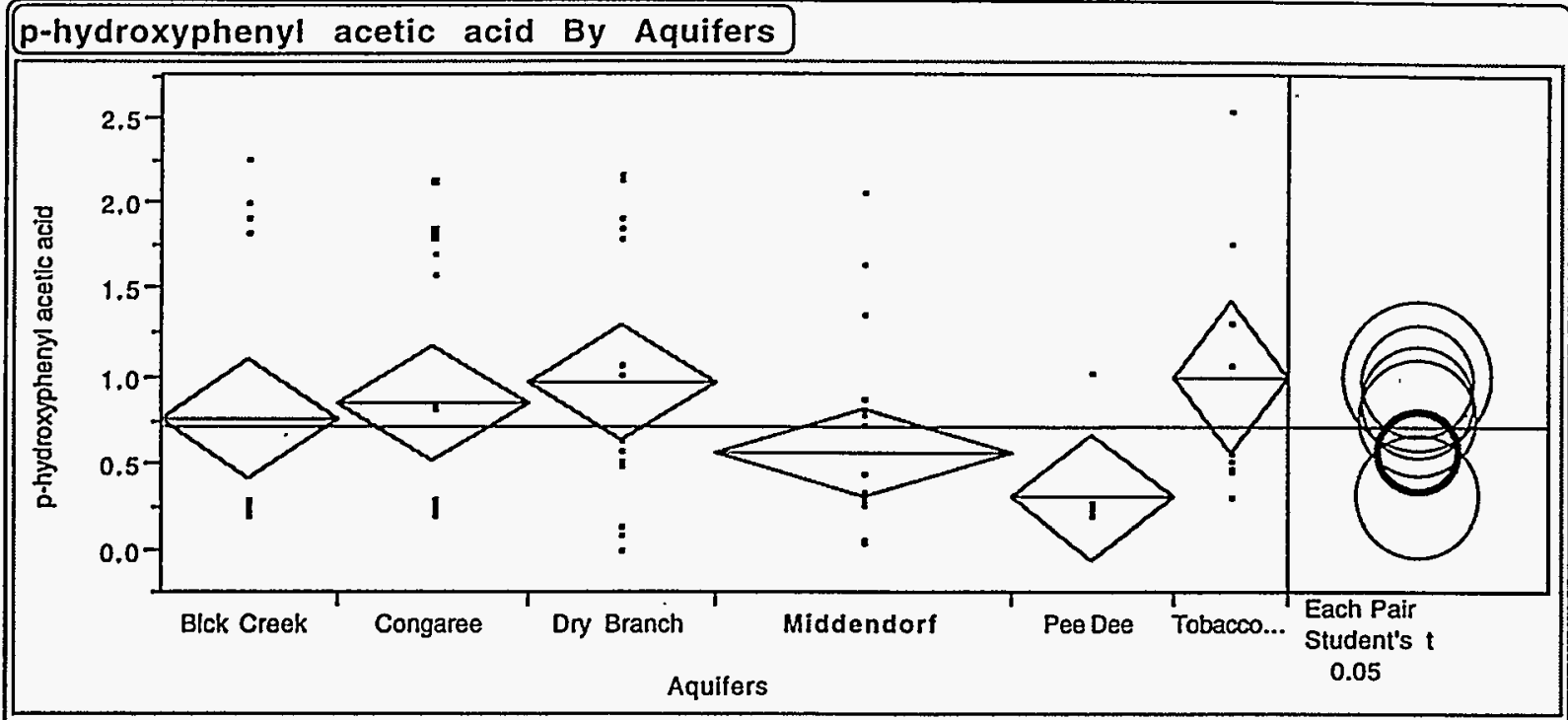

\section{Oneway Anova \\ Summary of Fit \\ RSquare}

RSquare Ad]

Root Mean Square Error

Mean of Response

Observations (or Sum Wgts)

Analysis of Variance

\begin{tabular}{|lrrrr|}
\hline Source & DF & Sum of Squares & Mean Square & F Ratio \\
Model & 5 & 4.701541 & 0.940308 & 2.1418 \\
Error & 84 & 36.877546 & 0.439018 & Prob>F \\
C Total & 89 & 41.579087 & & 0.0683 \\
\hline
\end{tabular}

Means for Oneway Anova

Level Number Mean Std Error

Blck Creek

Congaree

Dry Branch

McBean

Mlddendorf

Pee Dee

Tobacco Ro

$14 \quad 0.749643$

$15 \quad 0.841333$

150.957800

0

240.549708

130.304231

90.997444

Std Error uses a pooled estimate of error variance

0.17708

0.17108

0.17108

0.13525

0.18377

0.22086
Means Comparisons

DIf=Mean[I]-Mean[]] Dry Branch

Dry Branch

Congaree

Blck Creok

McBean

Tobacco Rd

Middendorf

Pee Deo

0.000000

Congaree Blck Creek

$-0.11647$

0.116467

\subsection{7}

0.091690

$-0.20816$

0.000000

0.000000

0.039644

$-0.40809$

0.156111

0.247802

$-0.29162$

$-0.65357$

$-0.5371$

Alpha $=0.05$

Comparisons for each pair using Student's t $\mathbf{t}$ 1.98862 Abs(Dif)-LSD Dry Branch Congareo Blck Creek McBean Tobacco Rd Mlddendorf Peo Deo

$\begin{array}{rrr}\text { Dry Branch } & \text { Congaree } & \text { Blck Creek } \\ -0.48113 & -0.36466 & -0.28149 \\ -0.36466 & -0.48113 & -0.39796 \\ -0.28149 & -0.39796 & -0.49802 \\ -0.51592 & -0.39945 & -0.31515 \\ -0.02559 & -0.14206 & -0.24318 \\ 0.154277 & 0.037811 & -0.06209\end{array}$

McBean Tobacco Rd Middendorf Pee Dee

McBean Tobacco Rd Middendorf Pee Dee - $\quad 0.03964 \quad 0.408092$ - $\quad-0.15611$ $\begin{array}{r}-0.2478 \\ \hline\end{array}$

0.1999350 .445412

Positive values show palrs of means that are significantly different. 
FIGURE $12 \mathrm{n}$.

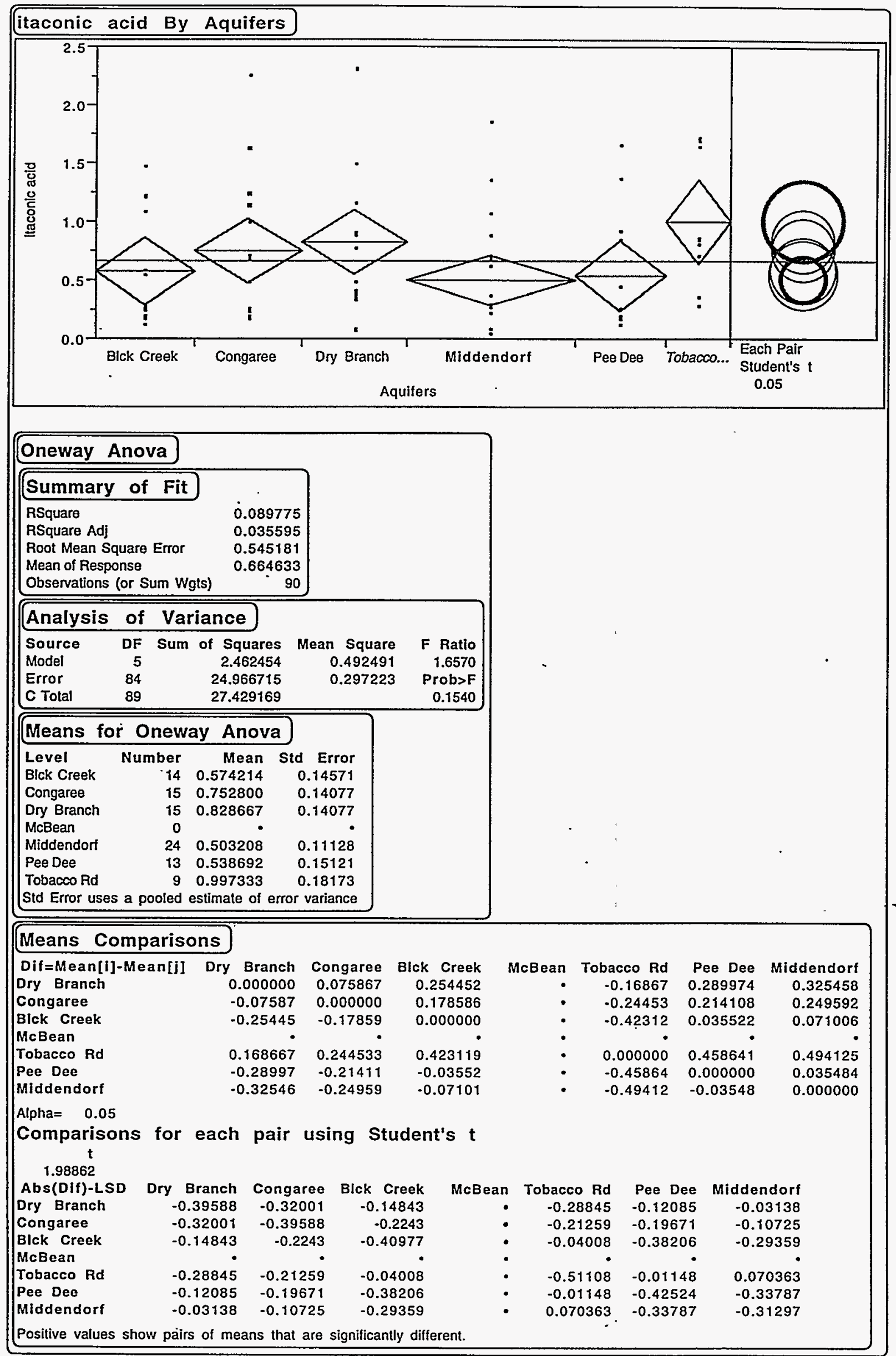




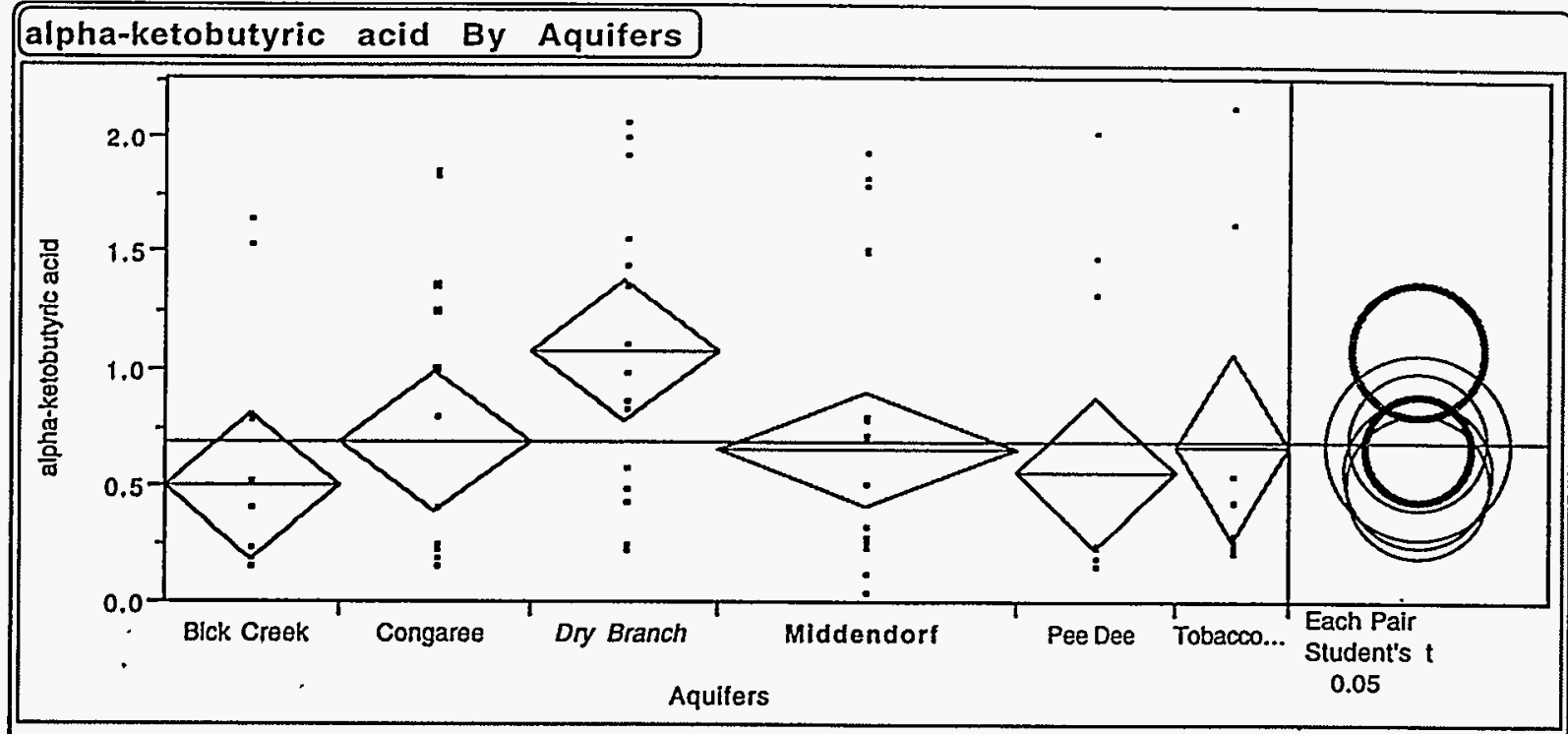

\section{Oneway Anova}

\section{Summary of Fit}

RSquare

RSquare Ad]

Root Mean Square Error

0.035377

Mean of Response

0.605774

Observations (or Sum Wgts)

0.688211

\begin{tabular}{|lrrrrr|}
\hline Analysis & of & Variance & & \\
Source & DF & Sum of Squares & Mean Square & F Ratio \\
Model & 5 & 3.032581 & 0.606516 & 1.6528 \\
Error & 84 & 30.824806 & 0.366962 & Prob $>$ F \\
C Total & 89 & 33.857387 & & 0.1551 \\
\hline
\end{tabular}

Means for Oneway Anova

Level Number Mean Std Error

$\begin{array}{llll}\text { Blck Creek } & 14 & 0.49657 & 0.16190\end{array}$

$\begin{array}{llll}\text { Congaree } & 15 & 0.68687 & 0.15641\end{array}$

$\begin{array}{llll}\text { Dry Branch } & 15 & 1.07247 & 0.15641\end{array}$

McBean

Middendorf

Pee Dee

$\begin{array}{rr}15 & 1.07247 \\ 0 & \text {. }\end{array}$

Tobacco Rd

$\begin{array}{ll}24 & 0.64675 \\ 13 & 0.54792\end{array}$

0.12365

$\begin{array}{ccc}9 & 0.66133 & 0.20192\end{array}$

Means Comparisons

DIf=Mean[I]-Mean[j] Dry Branch

Dry Branch

Congareo

Blck Creek

0.000000

$-0.3856$

Congaree Blck Creek

$-0.5759$

0.385600

0.575895

ddendorf Pee Dee $0.000000 \quad 0.190295$

$-0.1903$

0.000000

Tobacco Rd

Middendorf

$-0.41113$

$-0.42572$

$-0.02553$

Pee Dee

$-0.52454$

$-0.04012$

0.164762

0.150179

0.051352

\begin{tabular}{rrr} 
McBean Tobacco Rd Middendorf \\
\hline$\quad 0.411133$ & 0.425717
\end{tabular}

- 0.025533

$-0.16476$

-

- 0.000000

- $\quad-0.01458$

- -0.11341
0.040117

$-0.15018$

0.014583

0.000000

$-0.09883$

Comparisons for each pair using Student's t $t$

$$
1.98862
$$

Abs(DIf)-LSD

Dry Branch

Congareo

Blck Creek

McBean

Tobacco Rd

Middendorf

Pee Dee

Dry Branch
-0.43988
-0.05428
0.128232
-0.09679
0.029217
0.068062

Congareé

$-0.05428$

Blck Creek

$-0.43988$

0.128232

$-0.25737$

$-0.25737$

$-0.45532$

$-0.48239$

$-0.34992$

$-0.35638$

$-0.31754$

-0.25494
-0.41264
McBean Tobacco Rd Middendorf

- 0.09679

- $\quad-0.48239$

$-0.34992$

- 0.34992

- $\quad-0.56788$

- $\quad 0.45628$

$-0.40896$
0.029217

$-0.35638$

$-0.25494$

$-0.34775$

$-0.31602$
$-0.45628$
Pee Dee

0.068062

$-0.31754$

$-0.41264$

$-0.40896$

$-0.31602$

$-0.4725$

Positlve values show pairs of means that are significantly different 
FIGURE $12 p$.

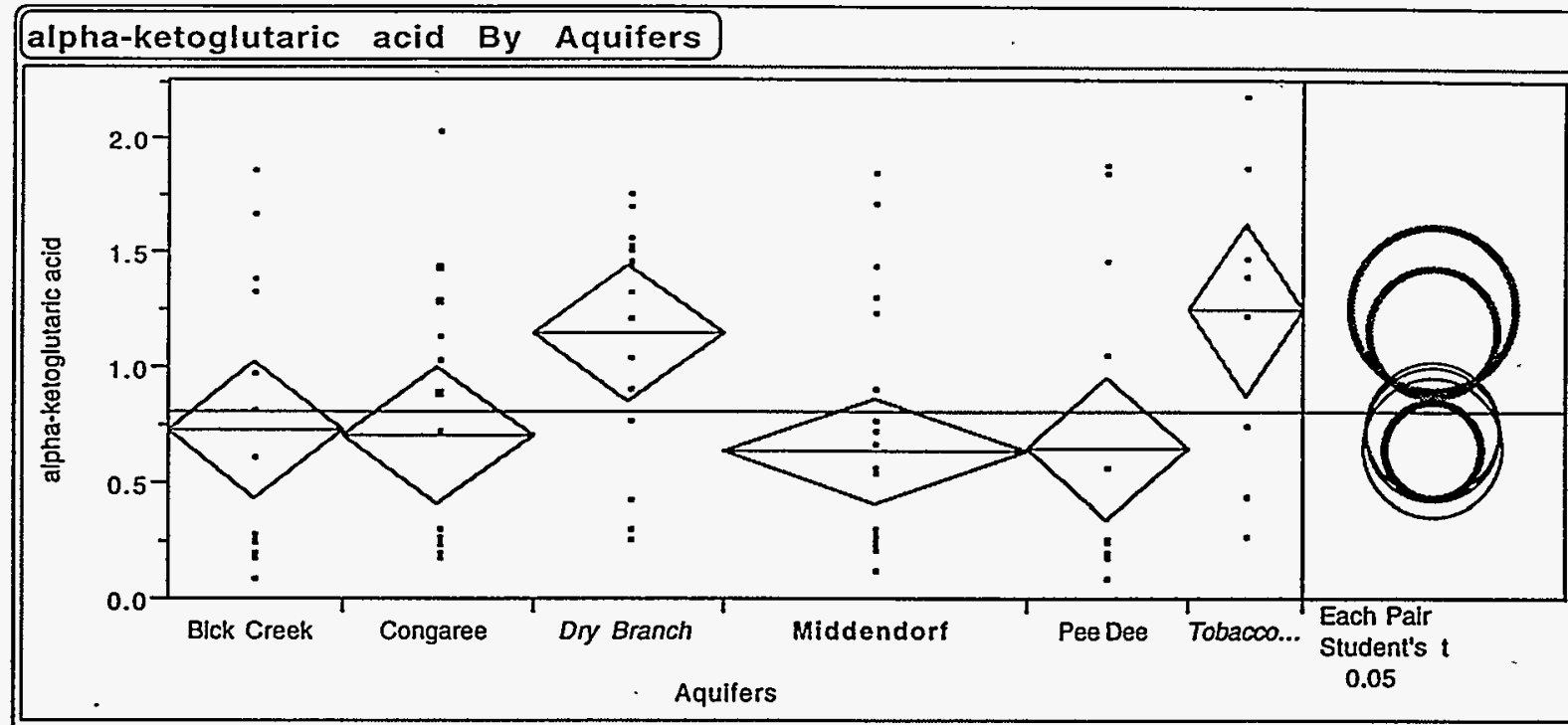

Oneway Anova

Summary of -Fit

RSquare

RSquare Adj

Root Mean Square Error

Mean of Response

Observations (or Sum Wgts)

0.146148

0.095324

0.573784

0.812911

\begin{tabular}{|lrrrr|}
\hline Analysis & of & Variance & & \\
Source & DF & Sum of Squares & Mean Square & F Ratio \\
Model & 5 & 4.733543 & 0.946709 & 2.8755 \\
Error & 84 & 27.655131 & 0.329228 & Prob>F \\
C Total & 89 & 32.388673 & & 0.0191 \\
\hline
\end{tabular}

Means for Oneway Anova

Level Number Mean std Error

$\begin{array}{llll}\text { Blck Creek } & 14 & 0.73064 & 0.15335\end{array}$

$\begin{array}{llll}\text { Congaree } & 15 & 0.73064 & 0.15335 \\ & 15 & 0.70333 & 0.14815\end{array}$

Dry Branch $\quad 15 \quad 1.15007 \quad 0.14815$

McBean

Middendorf

Pee Dee

Tobacco Rd

0.6434

0.64938

1.24967

0.11712

0.15914

0.19126

Std Error uses a pooled estimate of error variance

\section{Means Comparisons}

Dif=Mean[i]-Mean[j] Dry Branch Dry Branch

Blck Creek

Congaree

McBean

Tobacco Rd

Pee Dee

Middendorf

Alpha $=0.05$

Comparisons for each pair using Student's t

$$
1.98862
$$

Abs(Dif)-LSD

Dry Branch

Blck Creek

Congaree

McBean

Tobacco Rd

Pee Dee

Middendorf
Dry Branch
-0.41665
$-0.0046$
0.030086
$-0.3815$
0.068306

0.131047
Blck Creek Congaree 0.000000

$-0.41942$

$-0.44673$

0.099600

$-0.50068$

$-0.50661$
$0.419424 \quad 0.446733$

$0.000000 \quad 0.027310$

$\begin{array}{ll}-0.02731 & 0.000000\end{array}$

$0.519024 \quad 0.546333$

$\begin{array}{ll}-0.08126 & -0.05395\end{array}$

$\begin{array}{ll}-0.08718 & -0.05987\end{array}$

\section{McBean Tobacco Rd} $-0.0996$

$-0.51902$

$-0.54633$

0.000000

$-0.60028$

$-0.60621$
Pee Dee Middendorf

0.500682

0.081258

0.053949

0.600282

0.000000

$-0.00593$
0.506608

0.087185

0.059875

0.606208

0.005926

0.000000

Positive values show pairs of means that are significantly different. 
FIGURE 129.

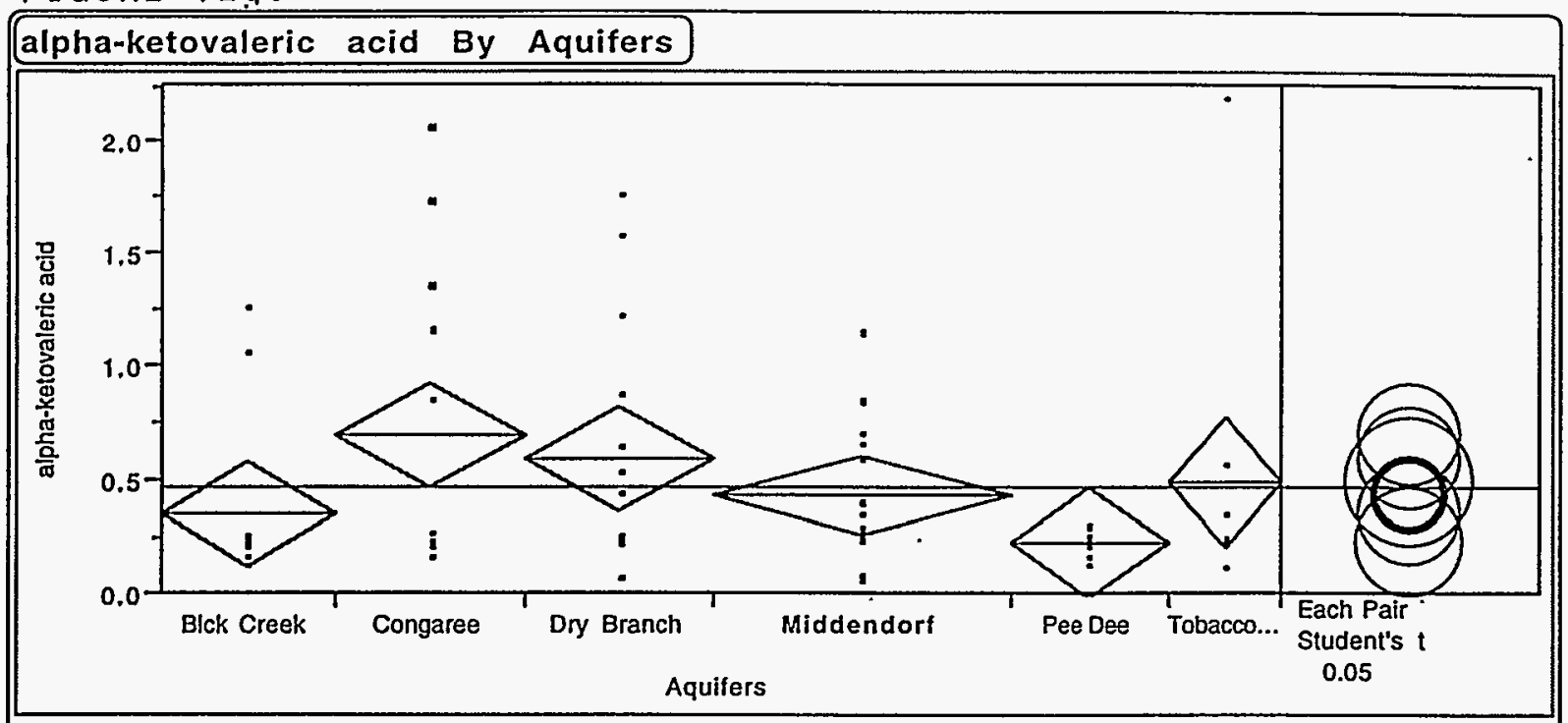

\section{Oneway Anova}

\begin{tabular}{|lr|}
\hline Summary of Fit & \\
\hline RSquare & 0.104358 \\
RSquare Ad] & 0.051046 \\
Root Mean Square Error & 0.449336 \\
Mean of Response & 0.463067 \\
Observations (or Sum Wgts) & 90 \\
\hline
\end{tabular}

\begin{tabular}{|lrrrr|}
\hline Analysis & of & Variance & & \\
Source & DF & Sum of Squares & Mean Square & F Ratio \\
Model & 5 & 1.976111 & 0.395222 & 1.9575 \\
Error & 84 & 16.959832 & 0.201903 & Prob>F \\
C Total & 89 & 18.935944 & & 0.0934 \\
\hline
\end{tabular}

\begin{tabular}{|c|c|c|c|}
\hline \multicolumn{4}{|c|}{ Means for Oneway Anova } \\
\hline Lovel & $\begin{array}{r}\text { Number } \\
14\end{array}$ & $\begin{array}{r}\text { Mean } \\
0.354500\end{array}$ & $\begin{array}{r}\text { Std Error } \\
0.12009\end{array}$ \\
\hline & 15 & 0.69 & 0.1 \\
\hline & 15 & 0.58 & 0.11 \\
\hline & 0 & & \\
\hline dorf & 24 & 0.426 & 0.0917 \\
\hline & 13 & 0.220 & 0.1246 \\
\hline ח & 9 & 0.492667 & 0.14978 \\
\hline
\end{tabular}

\begin{tabular}{|c|c|c|c|c|c|c|c|}
\hline \multicolumn{8}{|c|}{ Means Comparisons } \\
\hline $\begin{array}{l}\text { Dif = Mean }[I]-\text { Mean }[j] \\
\text { Congaree }\end{array}$ & $\begin{array}{r}\text { Congaree } \\
0.000000\end{array}$ & $\begin{array}{r}\text { Dry Branch } \\
0.101600\end{array}$ & $\begin{array}{r}\text { Blck Creek } \\
0.335500\end{array}$ & McBean & $\begin{array}{r}\text { Tobacco Rd } \\
0.197333\end{array}$ & $\begin{array}{r}\text { Middendorf } \\
0.263667\end{array}$ & $\begin{array}{l}\text { Pee Dee } \\
0.469154\end{array}$ \\
\hline Dry Branch & -0.1016 & 0.000000 & 0.233900 & - & 0.095733 & 0.162067 & 0.367554 \\
\hline Blck Creek & -0.3355 & -0.2339 & 0.000000 & - & -0.13817 & -0.07183 & 0.133654 \\
\hline McBean & - & - & - & - & - & - & • \\
\hline Tobacco Rd & -0.19733 & -0.09573 & 0.138167 & - & 0.000000 & 0.066333 & 0.271821 \\
\hline Middendorf & -0.26367 & -0.16207 & 0.071833 & - & -0.06633 & 0.000000 & 0.205487 \\
\hline Pee Dee & -0.46915 & -0.36755 & -0.13365 & - & -0.27182 & -0.20549 & 0.000000 \\
\hline
\end{tabular}

Alpha $=0.05$

Comparisons for each pair using Student's $t$ $t$

1.98862

Abs(DIf)-LSD

Congaree

Dry Branch

Blck Creek

McBean

Tobacco Rd

Middendort

Pee Dee

Congaree

Bry Branch Blck Creek

$-0.32628$

$-0.22468$

$-0.22468 \quad 0.003444$

$\begin{array}{ll}-0.22468 & 0.003444 \\ -0.32628 & -0.09816\end{array}$

0.003444

$-0.09816-0.33773$

$-0.17942$

$-0.03044$

$-0.28102$

$-0.13204$

0

$-0.22867$

$-0.21051$

McBean Tobacco Rd

$$
\begin{array}{r}
-0.17942 \\
-0.28102
\end{array}
$$

$-0.2436$

$-0.42123$

$-0.28293$

$-0.11565$
Middendorf

Pee Dee

$\begin{array}{ll}-0.03044 & 0.130556\end{array}$

$\begin{array}{ll}-0.13204 & 0.028956\end{array}$

$\begin{array}{lll}-0.22867 & -0.21051\end{array}$

$-0.28293 \quad-0.11565$

$-0.25795-0.10223$

$\begin{array}{ll}-0.10223 & -0.35048\end{array}$

Positive values show pairs of means that are signilicantly different. 
FIGURE $12 r$.

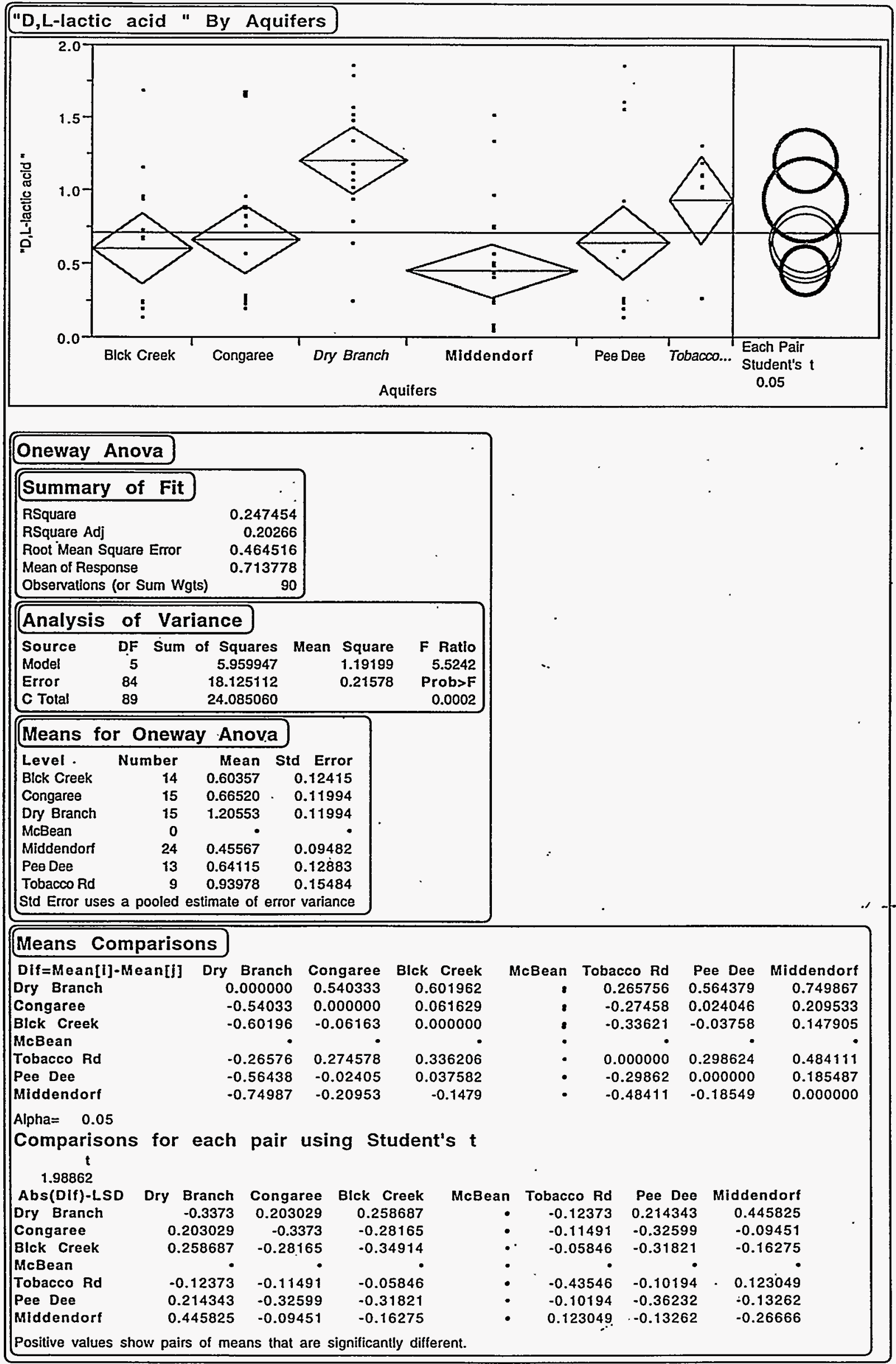


FIGURE - $12 \mathrm{~s}$.

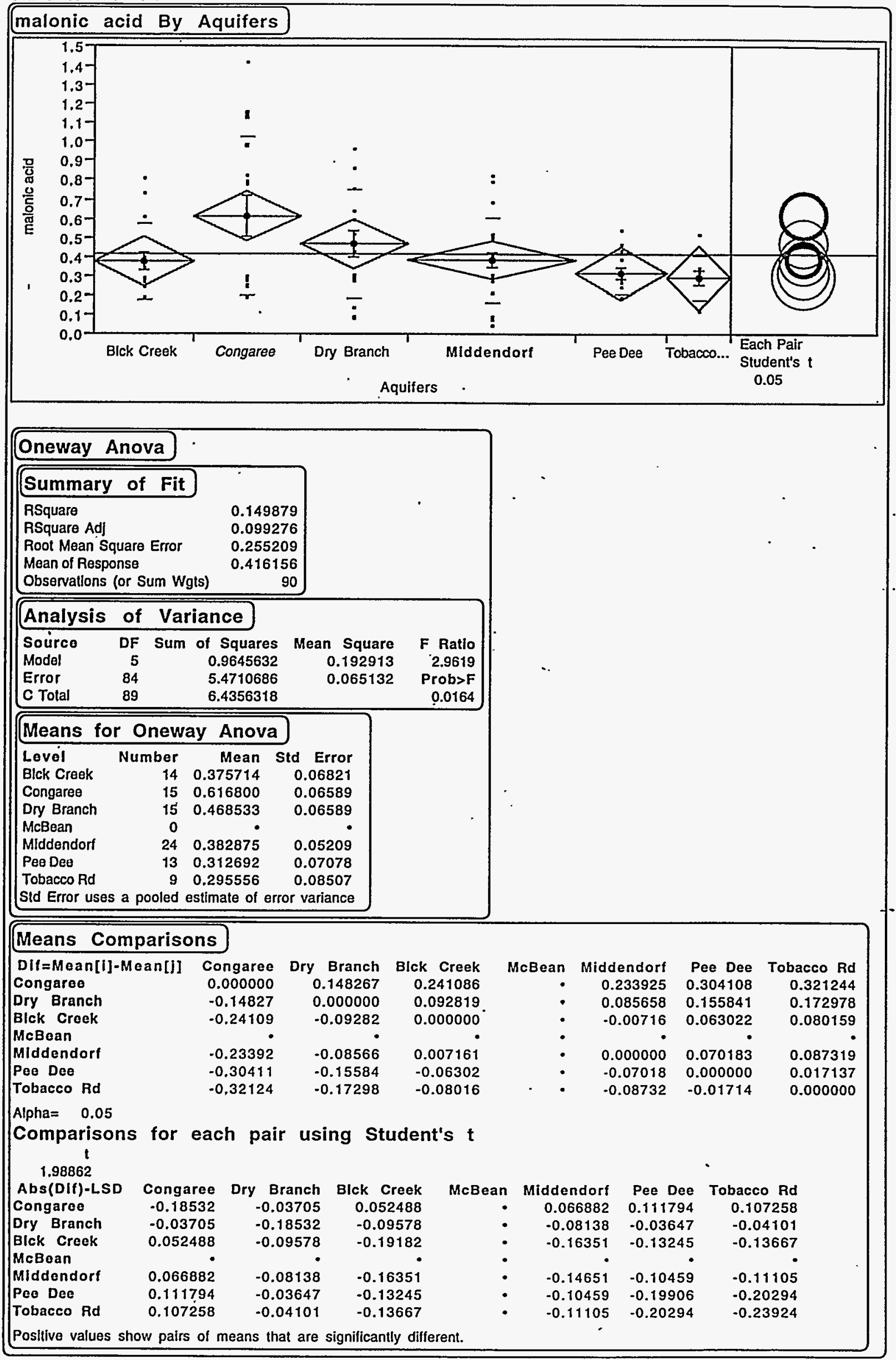


FIGURE $12 t$.

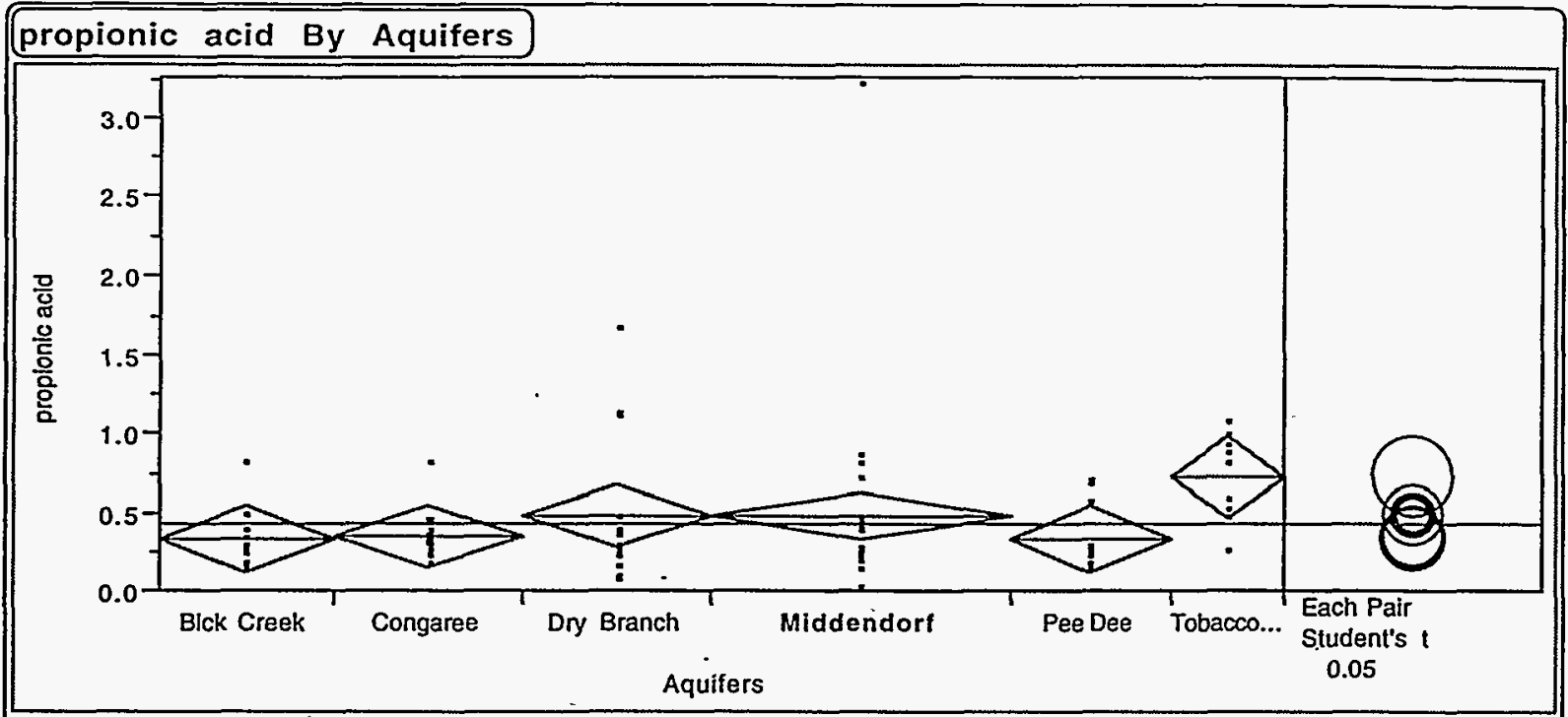

\section{Oneway Anova}

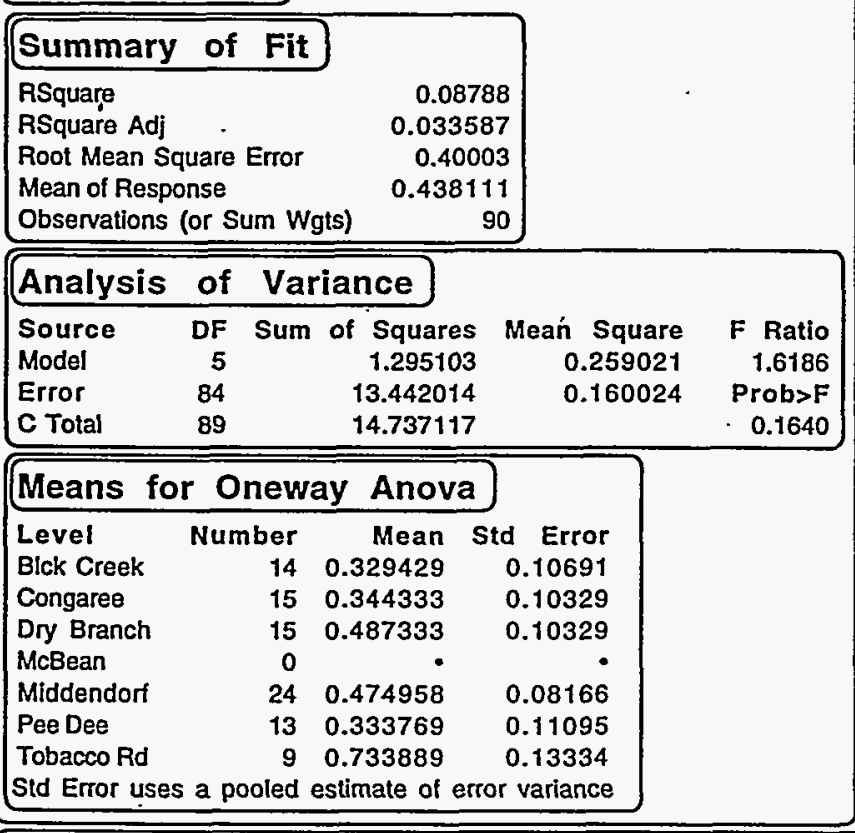

\section{Means Comparisons}

Dif=Mean[i]-Mean[i] Dry Branch Middendorf Blck Creek Dry Branch

Middendorf

0.157905

McBean Tobacco Ro Congaree

Pee Dee Blck Creek

McBean

Tobacco Rd

0.000000

0.145530

$-0.1579-0.14553$

Congaree

Pee Dee

0.246556

$-0.143$

0.258931

0.000000

0.404460

$-0.13063 \quad 0.014905$

$-0.14119$

0.004341 $-0.24656$

$-0.25893$

$-0.40446$

0.000000

$-0.38956$

$-0.40012$
0.143000

0.130625

$-0.0149$

0.389556

0.000000

$-0.01056$
0.153564

0.141189

$-0.00434$

0.400120

0.010564

0.000000

Alpha $=0.05$

Comparisons for each pair using Student's $t$

$t$

1.98862

Abs(Dif)-LSD Dry Branch Middendorf Blak Creek

Middendor

$-0.29048$

$\begin{array}{rrr}-0.24946 & \text { Blck Creek } \\ -0.13771\end{array}$

$-0.24946-0.22964$

$-0.122$

$-0.13771$

$-0.122$

$-0.30067$

McBean

Tobacco Rd

$-0.08886$

$-0.14748$

$-0.05201$

$-0.13121$

$-0.14788$

$-0.13276$

0.064583

$-0.28071$

$-0.30206$

McBean Tobacco Rd Congaree

Pee Dee

Pee Dee

Positive values show pairs of means that are significantly different.

$-0.14788$

$-0.13276$

$-0.30206$

0.055165

$-0.29088$

$-0.31202$ 
FIGURE' $12 u$.

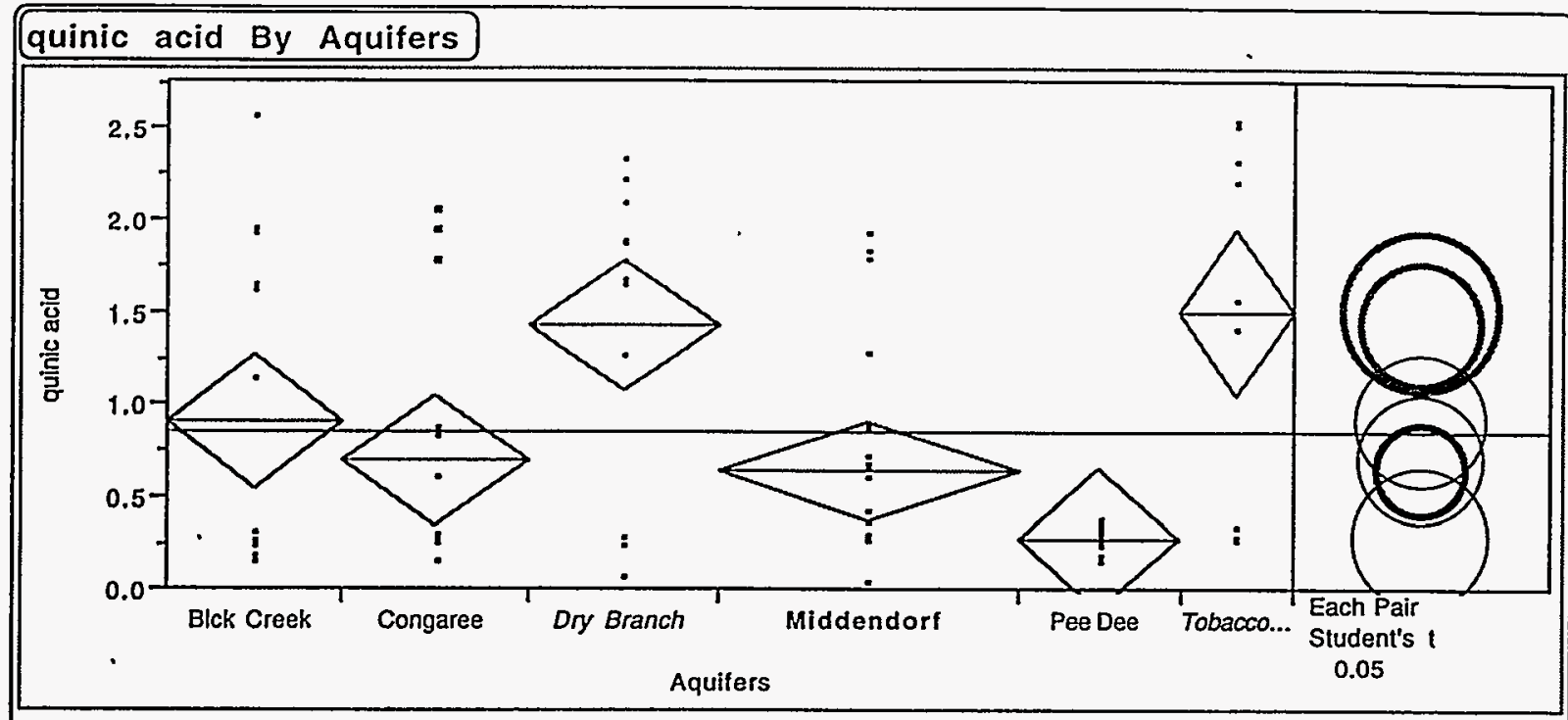

\section{Oneway Anova}

Summary of Fit

RSquare

RSquare Ad]

Root Mean Square Error

Mean of Response

Observations (or Sum Wgts)

0.222735

0.688154

0.853111

90

\begin{tabular}{|c|c|c|c|c|c|}
\hline \multicolumn{6}{|c|}{ Analysis of Variance } \\
\hline $\begin{array}{l}\text { Source } \\
\text { Model. } \\
\text { Error } \\
\text { C Total }\end{array}$ & Sum & $\begin{array}{r}\text { of Squares } \\
14.445362 \\
39.778703 \\
54.224065\end{array}$ & Mean & $\begin{array}{l}\text { Square } \\
2.88907 \\
0.47356\end{array}$ & $\begin{array}{r}\text { F Ratio } \\
.6 .1008 \\
\text { Prob>F } \\
0.0001\end{array}$ \\
\hline \multicolumn{6}{|c|}{ Means for Oneway Anova } \\
\hline $\begin{array}{l}\text { Level } \\
\text { Btck Creek } \\
\text { Congaree } \\
\text { Dry Branch } \\
\text { McBean } \\
\text { Middendorf } \\
\text { Pee Dee } \\
\text { Tobacco Rd } \\
\text { Std Error uses a po }\end{array}$ & $\begin{array}{r}\text { Iber } \\
14 \\
15 \\
15 \\
0 \\
24 \\
13 \\
9 \\
\text { oled es }\end{array}$ & $\begin{array}{c}\text { Mean S } \\
0.90957 \\
0.68987 \\
1.41940 \\
. \\
0.63967 \\
0.27462 \\
1.49833 \\
\text { stimate of err }\end{array}$ & $\begin{array}{r}\text { Std Err } \\
0.183 \\
0.177 \\
0.177 \\
\\
0.140 \\
0.190 \\
0.229 \\
\text { ror varian }\end{array}$ & $\begin{array}{l}\text { ror } \\
92 \\
68 \\
68 \\
4 \\
47 \\
86 \\
38 \\
\text { nce }\end{array}$ & \\
\hline
\end{tabular}

\section{Means Comparisons}

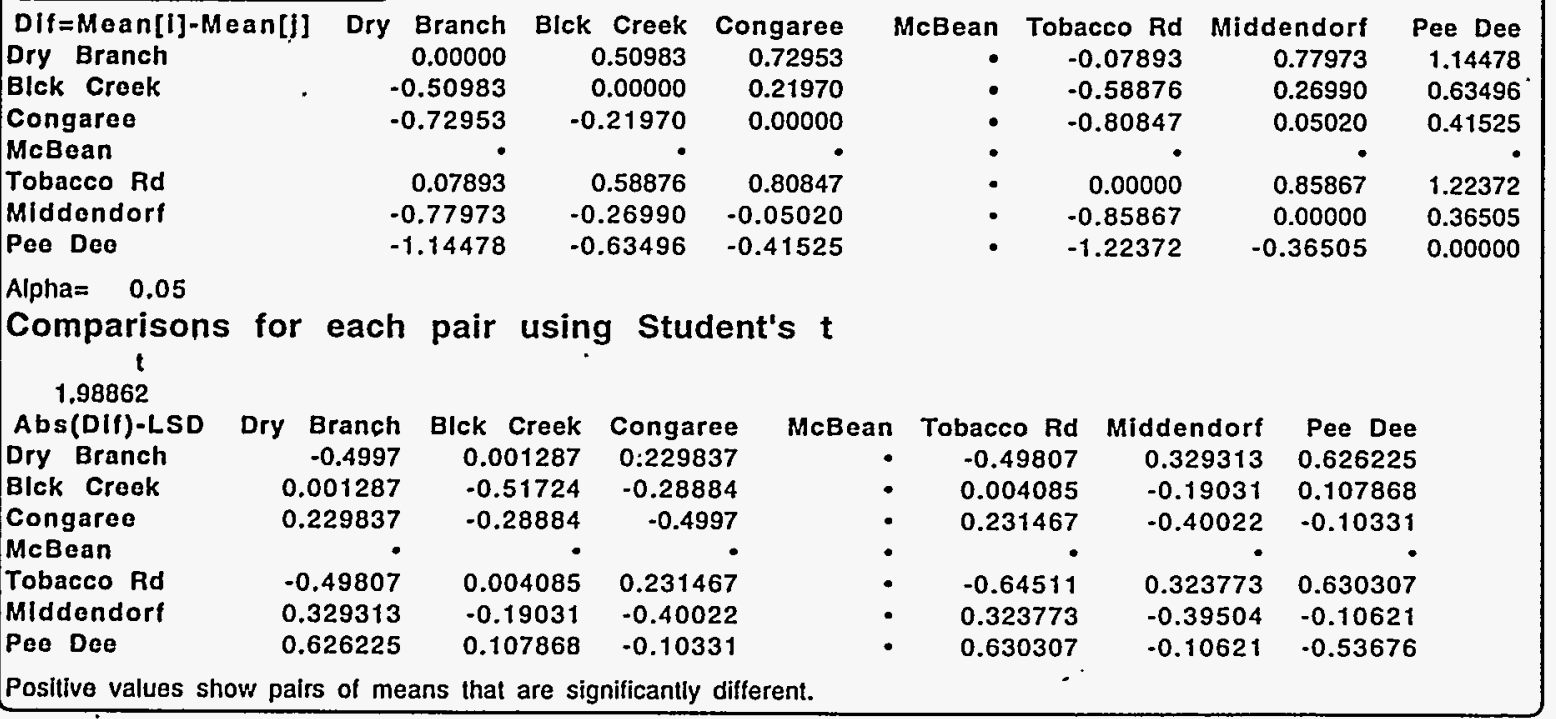




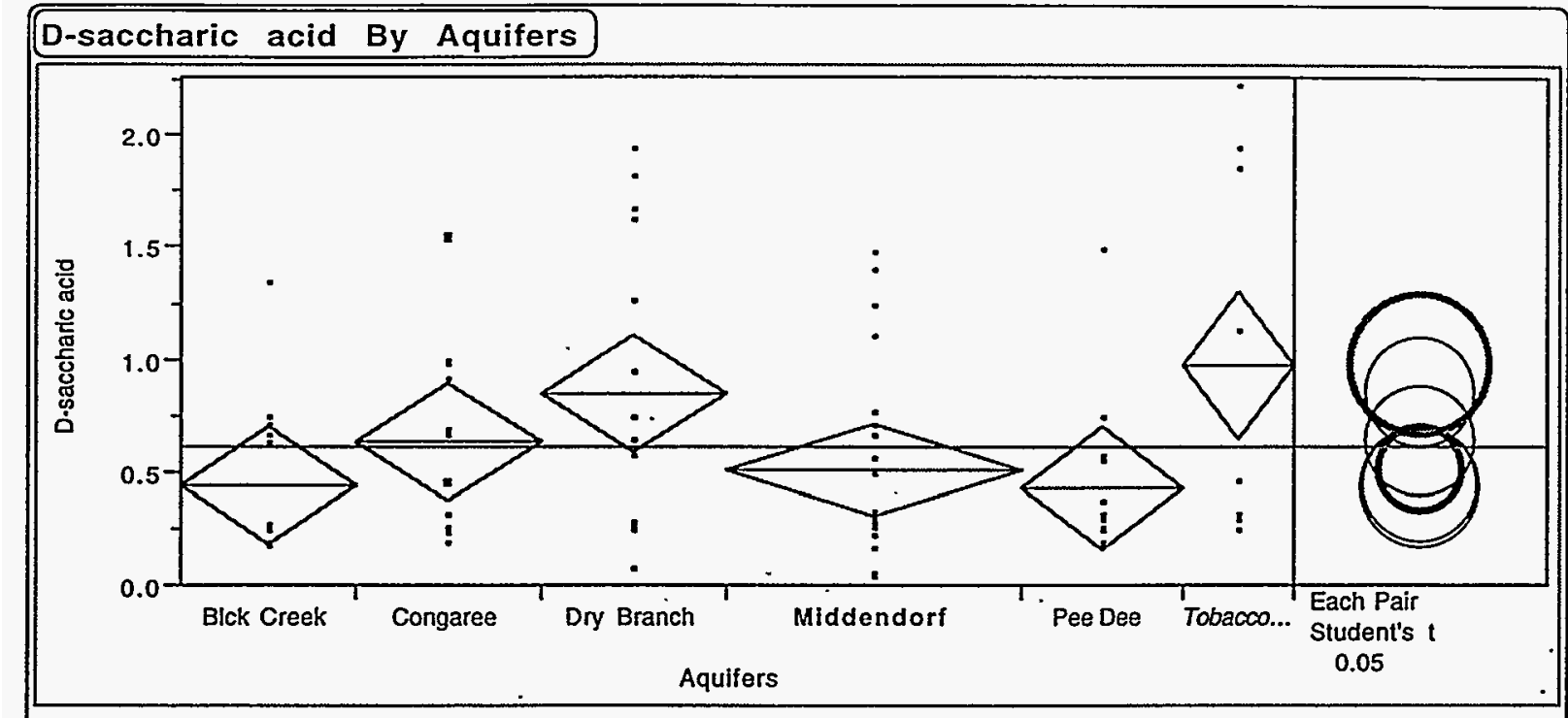

\section{Oneway Anova}

\section{Summary of Fit}

RSquare

RSquare Adj

Root Mean Square Error

Mean of Response

Observations (or Sum Wgts)

$\begin{array}{r} \\ 0.123233 \\ 0.071044 \\ 0.503975 \\ 0.6147 \\ 90 \\ \hline\end{array}$

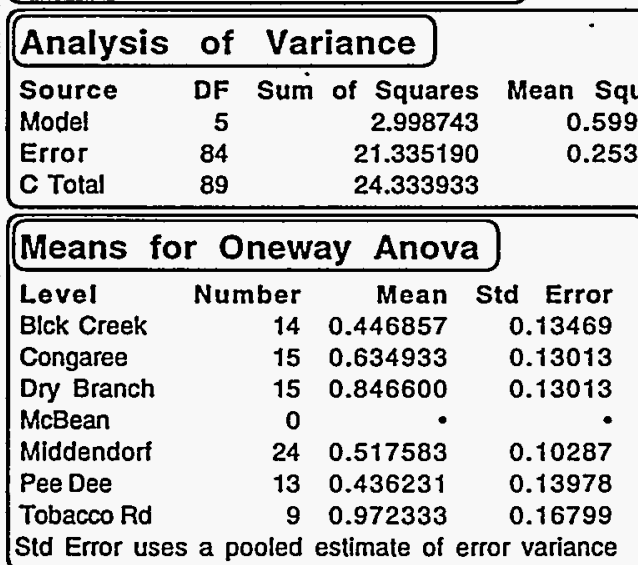

\section{Means Comparisons}

$\begin{array}{lrrr}\text { Dif=Mean[i]-Mean[]] } & \text { Dry Branch } & \text { Congaree } & \text { Blck Creek } \\ \text { Dry Branch } & 0.000000 & 0.211667 & 0.399743 \\ \text { Congaree } & -0.21167 & 0.000000 & 0.188076 \\ \text { Blck Creek } & -0.39974 & -0.18808 & 0.000000 \\ \text { McBean } & - & - & \\ \text { Tobacco Rd } & 0.125733 & 0.337400 & 0.525476 \\ \text { Middendorf } & -0.32902 & -0.11735 & 0.070726 \\ \text { Pee Dee } & -0.41037 & -0.1987 & -0.01063\end{array}$

$$
\begin{array}{rrrr}
\text { McBean } & \text { Tobacco Rd } & \text { Middendorf } & \text { Pee Dee } \\
- & -0.12573 & 0.329017 & 0.410369 \\
\dot{*} & -0.3374 & 0.117350 & 0.198703 \\
\dot{-} & -0.52548 & -0.07073 & 0.010626 \\
\dot{-} & 0.000000 & 0.454750 & 0.536103 \\
- & -0.45475 & 0.000000 & 0.081353 \\
& -0.5361 & -0.08135 & 0.000000
\end{array}
$$

Alpha $=0.05$

Comparisons for each pair using Student's $t$

$$
\text { t }
$$

$$
1.98862
$$

Abs(Dif)-LSD

Dry Branch

Congaree

Blck Creek

McBean

Tobacco Rd

MIddendorf

Pee Dee
Dry Branch Congaree
$-0.36596$
$-0.15429$
0.027309
$-0.36596$
$-0.18436$
$-0.29684$
$-0.00085$
0.030598
$-0.08517$
$-0.21252$
$-0.18107$
Blck Creek
0.027309
$-0.18436$
$-0.3788$
0.097284
$-0.26631$
$-0.37539$

McBean Tobacco Rd Middendorf

\section{- -0.29684}

$-0.08517$

0.097284

Positive values show pairs of means that are significantly different.

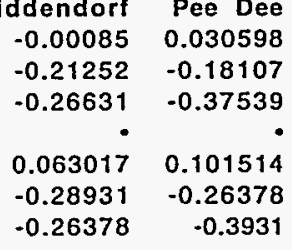


FIGURE $12 W$.

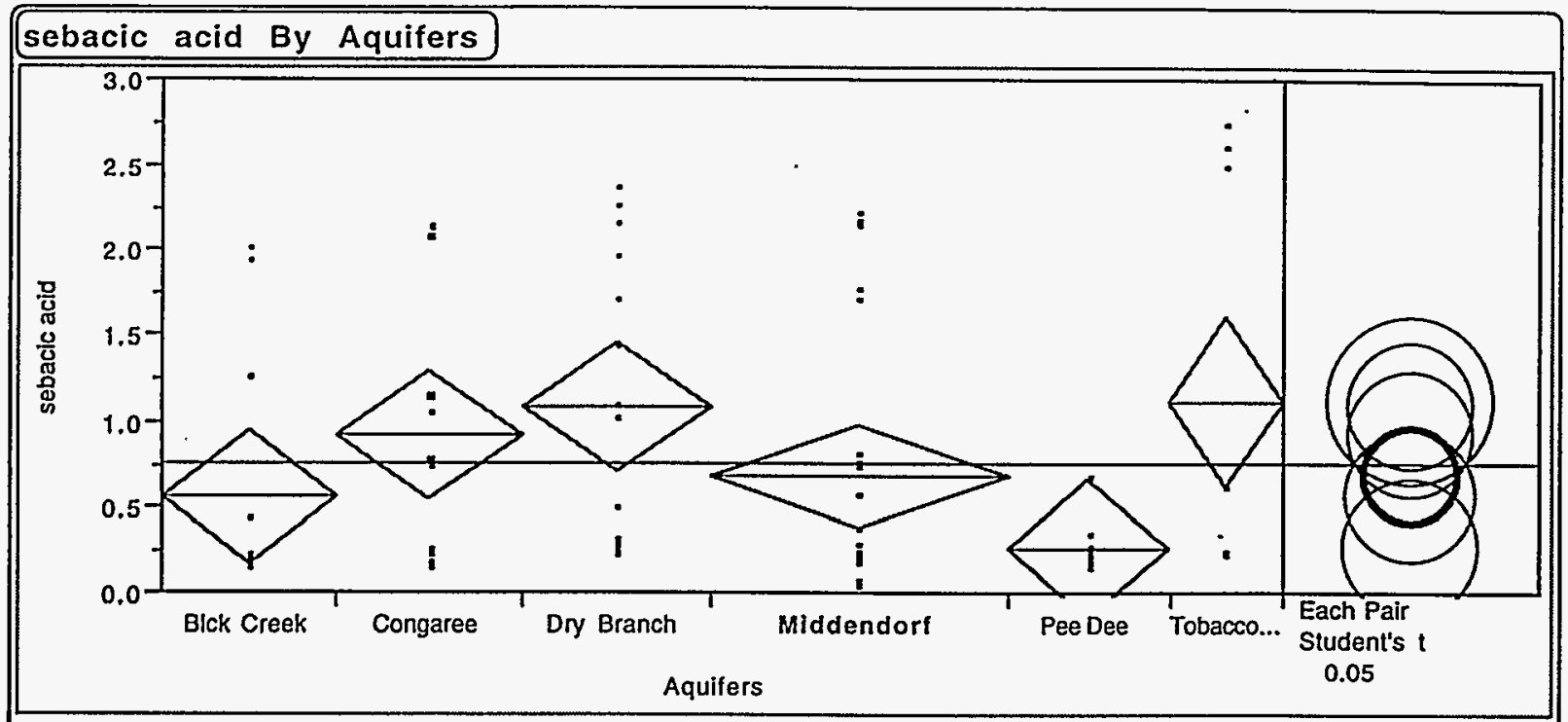

\section{Oneway Anova}

Summary of Fit

RSquare

RSquare Adj

Root Mean Square Error

Mean of Response

0.075666

0.755126

Observations (or Sum Wgts)

90

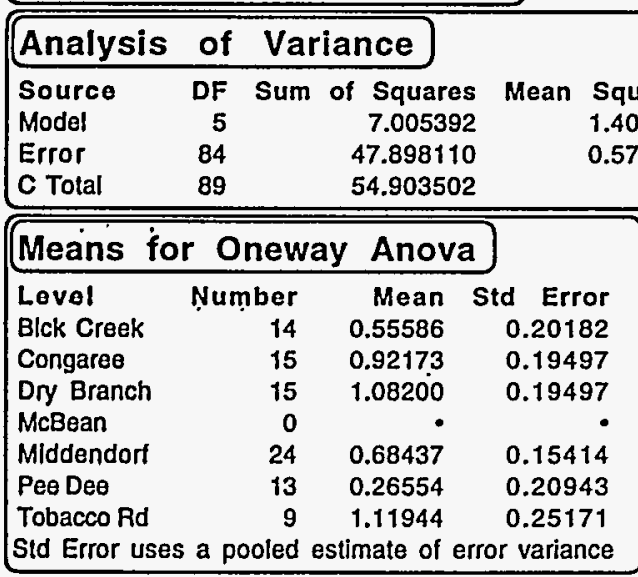

Means Comparisons

Dlf=Mean[i]-Mean[j] Dry Branch

Dry Branch

Congaree

Blck Creek

McBean

Tobacco Rd

Middendorf

Pee Deo

0.000000

$-0.16027$

$-0.52614$

Congaree

0.160267

Blck Creek

0.365876

$-0.36588$

0.000000

0.037444

$-0.39763$

0.197711

0.563587

$-0.23736$

0.128518

$\begin{array}{lll}-0.81646 & -0.65619 & -0.29032\end{array}$

\begin{tabular}{rrrr} 
McBean & Tobacco Rd & Middendorf & Pee Dee \\
- & -0.03744 & 0.397625 & 0.816462 \\
- & -0.19771 & 0.237358 & 0.656195 \\
- & 0.56359 & -0.12852 & 0.290319 \\
\hline & 0.000000 & 0.435069 & 0.853906 \\
$\cdot$ & -0.43507 & 0.000000 & 0.418837 \\
- & -0.85391 & -0.41884 & 0.000000
\end{tabular}

Alpha $=0.05$

Comparisons for each pair using Student's $t$

1.98862

Abs(DII)-LSD

Dry Branch

Congaree

Blck Creok

McBean

Tobacco Rd

Mlddendorf

Pee Deo

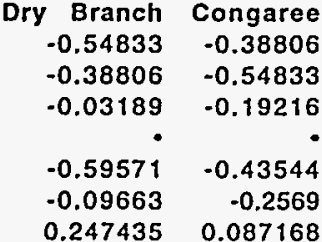

Congaree

Blck Creek

$-0.03189$

$-0.19216$

$-0.56757$

$-0.07799$

$-0.37648$

$-0.28807$
McBean Tobacco Rd Middendorf $-0.09663$

$-0.2569$

$-0.37648$

$-0.15188$

$-0.43349$

$-0.09829$
Pee Dee

0.247435

0.087168

$-0.28807$

0.202744

$-0.09829$

$-0.589$

Positive values show pairs of means that are significantly different. 
FIGURE $12 x$.

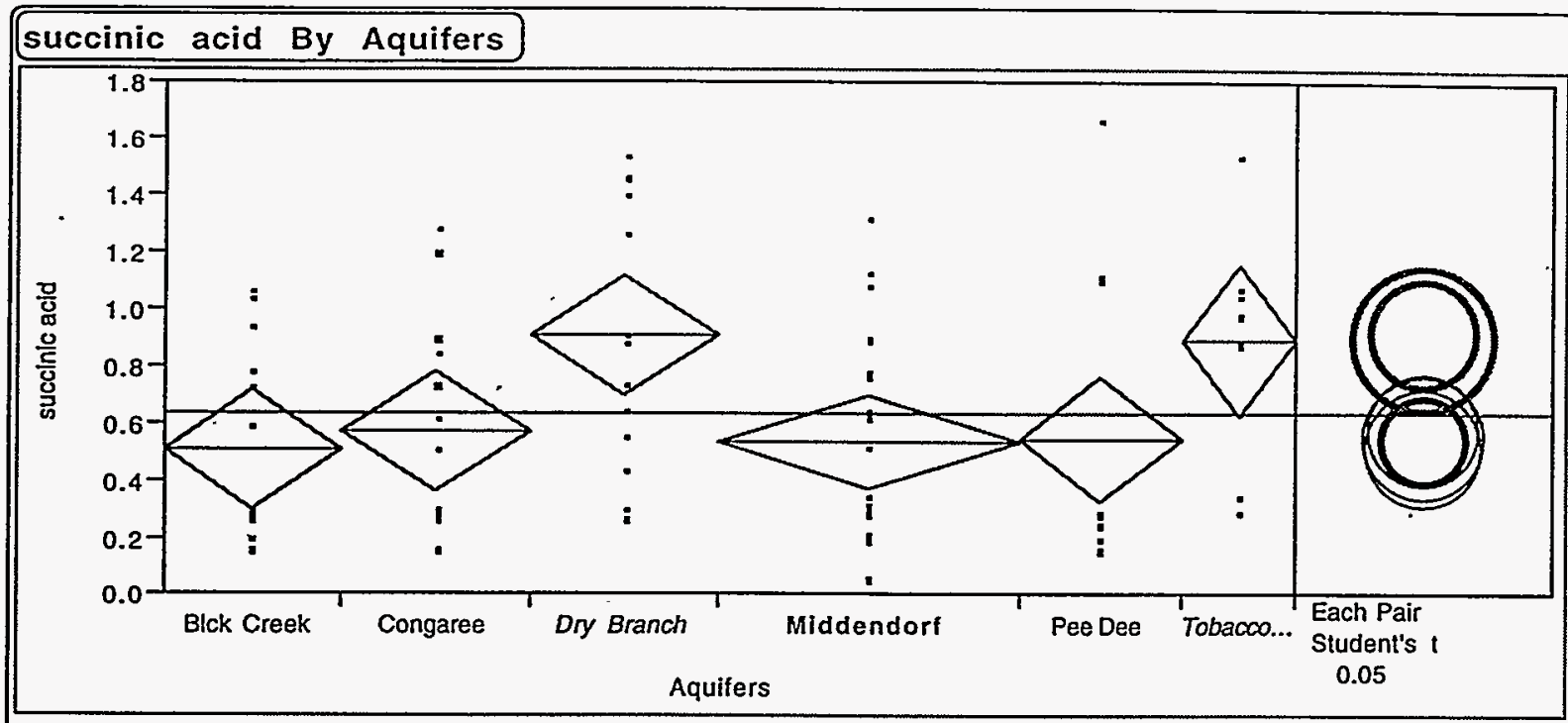

\section{Oneway Anova}

\section{Summary of Fit}

RSquare

RSquare Adj

Root Mean Square Error

Mean of Response

Observations (or Sum Wgts)

Analysis of Variance

\begin{tabular}{|lrrrrr}
\hline Source & DF & Sum of Squares & Mean Square & F Ratio \\
Model & 5 & 2.357348 & 0.471470 & 2.8083 \\
Error & 84 & 14.102469 & 0.167887 & Prob>F \\
C Total & 89 & 16.459817 & & 0.0215 \\
\hline
\end{tabular}

\begin{tabular}{|lrrrr}
\hline \multicolumn{3}{|l}{ Means for Oneway } & \multicolumn{3}{c|}{ Anova } \\
\cline { 1 - 4 } Level & Number & Mean & Std Error \\
Blck Creek & 14 & 0.506429 & 0.10951 \\
Congaree & 15 & 0.567133 & 0.10579 \\
Dry Branch & 15 & 0.909200 & 0.10579 \\
McBean & 0 &. & \\
Middendorf & 24 & 0.535125 & 0.08364 \\
Pee Dee & 13 & 0.540692 & 0.11364 \\
Tobacco Rd & 9 & 0.887556 & 0.13658 \\
Std Error uses a pooled estimate of error variance &
\end{tabular}

\section{Means Comparisons}

Dif=Mean[i]-Mean[j] Dry Branch

Dry Branch

Congaree

Blck Creek

McBean

Tobacco Rd

Pee Dee

Middendorf

Alpha $=0.05$

Comparisons for each pair using Student's $t$
Branch Congarèe Blck Creek

$=0.34207 \quad 0.000000$

$-0.40277$

$-0.02164$

$-0.36851$

$-0.37408$
0.402771

0.060705

.0 .000000

0.320422

$-0.02644$

$-0.03201$
0.381127

0.034264

0.028696
0.342067
McBean Tobacco Rd

- $\quad 0.021644$

- $\quad 0.32042$

- -0.38113

- 0.000000

-0.34686
$-\quad-0.35243$
Pee Dee

0.368508

0.026441

$-0.03426$

0.346863

0.000000

$-0.00557$
Middendorf 0.374075

0.032008 $-0.0287$

0.352431

0.005567

0.000000
1.98862

\section{Abs(Dif)-LSD}

Dry Branch

Congaree

Blck Creek

McBean

Tobacco Rd

Pee Dee

Middendorf

Dry Branch

Congaree

$-0.297530 .044538$

0.044538

$-0.29753$

$-0.24209$

$-0.023 i 3$

$0.059748 \quad-0.28232$

0.105886

$-0.23618$
Blck Creek 0.099976

$-0.24209$

$-0.30797$

0.033000

$-0.27957$

$-0.24532$
McBean Tobacco Rd $-0.32191$

$-0.02313$

0.033000

$-0.38411$

$-0.00646$

0.033945
Pee Dee Middendorf 0.059748

$-0.28232$

$-0.27957$

.0 .00646

$-0.3196$

$-0.27503$
0.105886

$-0.23618$

$-0.24532$

0.033945

.0 .27503

$-0.23522$

Positive values show pairs of means that are significantly different. 
FIGURE 13.

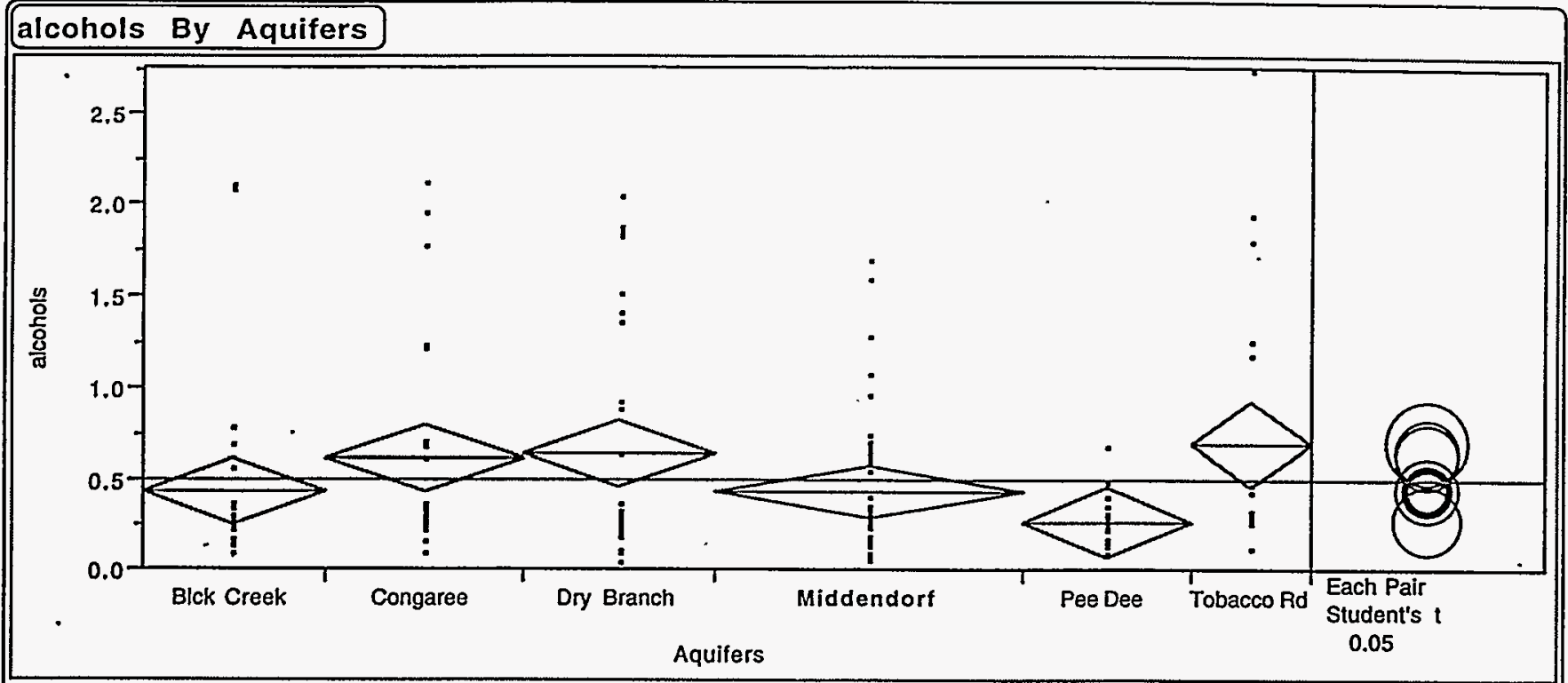

\section{Oneway Anova}

\section{Summary of Fit}

RSquare

RSquare Adj

Root Mean Square Error

Mean of Response

Observallons (or Sum Wgts)

0.041546

0.510374

0.496828

180

Analysis of Variance

\begin{tabular}{lrrrrr} 
Source & DF & Sum of Squares & Mean Square & F Ratio \\
Model & 5 & 3.323523 & 0.664705 & 2.5518 \\
Error & 174 & 45.323750 & 0.260481 & Prob $>$ F \\
C Total & 179 & 48.647274 & & 0.0295 \\
\hline
\end{tabular}

Means for Oneway Anova

Level Number Mean Std Error

$\begin{array}{llll}\text { Blck Creek } & 28 & 0.427179 & 0.09645\end{array}$

$\begin{array}{llll}\text { Congaree } & 30 & 0.608233 & 0.09318\end{array}$

$\begin{array}{llll}\text { Dry Branch } & 30 & 0.632267 & 0.09318\end{array}$

McBean

$\begin{array}{lrrr}\text { Middendorf } & 48 & 0.433833 & 0.07367\end{array}$

$\begin{array}{llll}\text { Pee Dee } & 26 & 0.266846 & 0.10009\end{array}$

$\begin{array}{llll}\text { Tobacco Rd } & 18 & 0.693944 & 0.12030\end{array}$

Sid Error uses a pooled estimate of error variance

\section{Means Comparisons}

\begin{tabular}{lrrrr}
\hline Dif=Mean[I]-Mean[j] & Dry Branch & Congaree & Blck Creek \\
Dry Branch & 0.000000 & 0.024033 & 0.205088 \\
Congaree & -0.02403 & 0.000000 & 0.181055 \\
Blck Creek & -0.20509 & -0.18105 & 0.000000 \\
McBean & - & $\bullet$ & \\
Tobacco Rd & 0.061678 & 0.085711 & 0.266766 \\
Mlddendorf & -0.19843 & -0.1744 & 0.006655 \\
Pee Dee & -0.36542 & -0.34139 & -0.16033
\end{tabular}

\section{McBean Tobacco Rd Middendorf Pee Dee \\ $0.198433 \quad 0.365421$ \\ $0.174400 \quad 0.341387$ \\ $-0.006650 .160332$ \\ $-0.08571$ \\ . \\ 0.000000 \\ $-0.26011$ \\ 0.260111 \\ $0.000000 \quad 0.166987$ \\ $-0.16699 \quad 0.000000$}

Alpha $=0.05$

Comparisons for each pair using Student's t

1.97371

Abs(Dif)-LSD

Dry Branch

Congaree

Blck Creek

McBean

Tobacco Ad

MIddendorf

Pee Dee

$\begin{array}{rrr}\text { Dry Branch } & \text { Congaree } & \text { Blck Creek } \\ -0.26009 & -0.23606 & -0.05961 \\ -0.23606 & -0.26009 & -0.08364 \\ -0.05961 & -0.08364 & -0.26922 \\ -0.23865 & -0.21462 & -0.03756 \\ -0.03601 & -0.06004 & -0.23289 \\ 0.095511 & 0.071477 & -0.11402\end{array}$

McBean Tobacco Rd Middendorf

$-0.23865$

$-0.21462$

$-0.03756$

$-0.33578$

$-0.0183$

0.118228 -0.03601

$-0.06004$

$-0.23289$

$-0.0183$

$-0.20562$

$-0.0783$
Pee Dee

0.095511

0.071477

$-0.11402$

0.118228

$-0.0783$

$-0.27938$

Posllive values show pairs of means that are signilicantly dilferent. 
FIGURE $13 \mathrm{a}$.

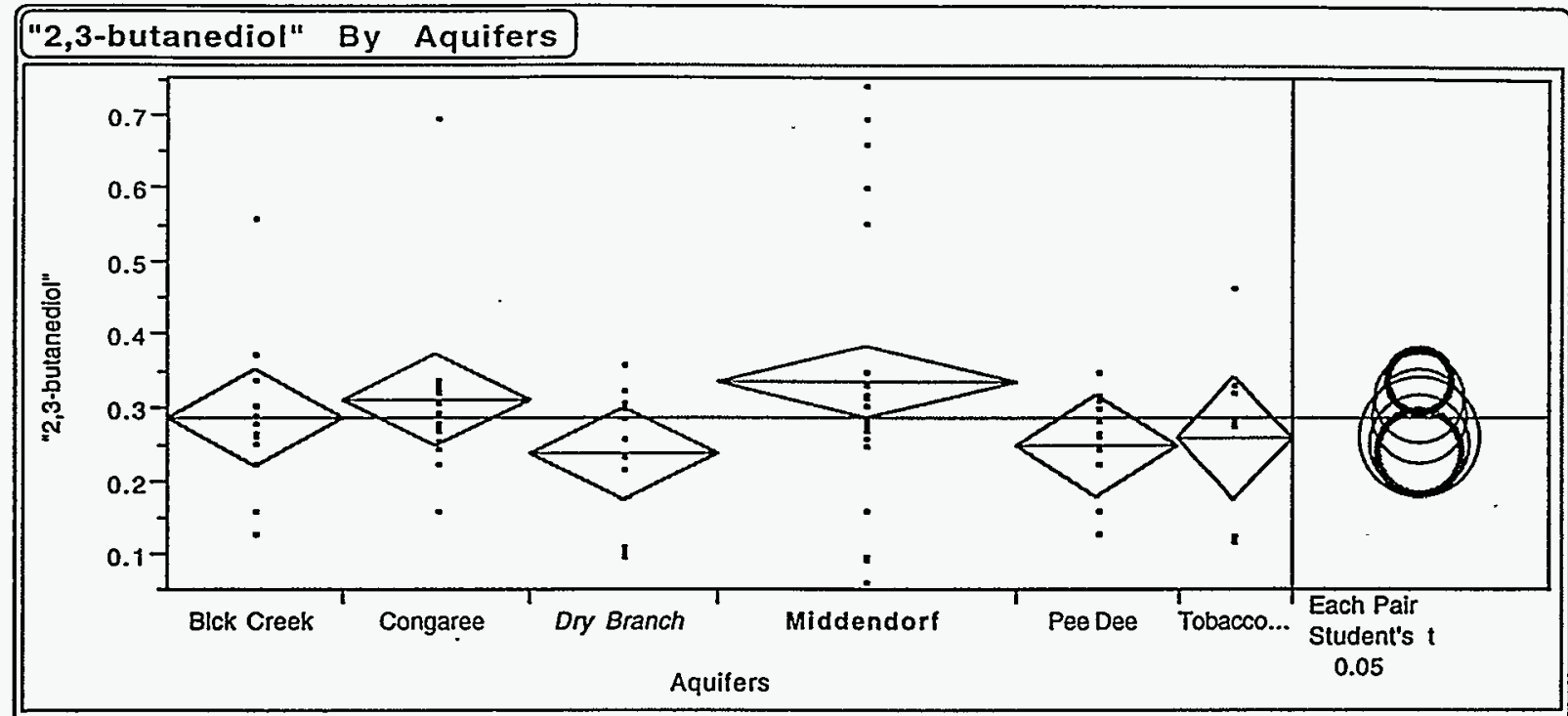

\section{Oneway Anova}

Summary of Fit

RSquare

RSquare Adj

Root Mean Square Error

Mean of Response

Observations (or Sum Wgts)

0.028212

0.128872

0.286833

90

\begin{tabular}{|lrrrrr|}
\hline Analysis & of & \multicolumn{2}{c|}{ Variance } & & \\
Source & DF & Sum of Squares & Mean Square & F Ratio \\
Model & 5 & 0.1259500 & 0.025190 & 1.5167 \\
Error & 84 & 1.3950665 & 0.016608 & Prob>F \\
C Total & 89 & 1.5210165 & & 0.1933 \\
\hline
\end{tabular}

Means for Oneway Anova

Level Number Mean Std Error

$\begin{array}{llll}\text { Blck Creek } & 14 & 0.284929 & 0.03444\end{array}$

$\begin{array}{llll}\text { Congaree } & 15 & 0.310667 & 0.03327\end{array}$

$\begin{array}{llll}\text { Dry Branch } & 15 & 0.239000 & 0.03327\end{array}$

McBean

$\begin{array}{rrrr}15 & 0.239000 & 0.03327\end{array}$

Middendorf $\quad \begin{array}{llll} & 24 & 0.335000 & 0.02631\end{array}$

$\begin{array}{llll}\text { Pee Dee } & 13 & 0.248000 & 0.03574\end{array}$

$\begin{array}{llll}\text { Tobacco Rd } & 9 & 0.257444 & 0.04296\end{array}$

Std Error uses a pooled estimate of error variance

\section{Means Comparisons}

Dif=Mean[i]-Mean[j] Middendorf Blck Creek Middendorf

Blck Creek

0.000000

Pee Dee 0.0500710 .087000

$0.000000,0.036929$

0.05007

$-0.03693 \cdot 0.000000$

Pee Dee

McBean

Congaree

Tobacco Rd

Dry Branch

.

$0.025738 \quad 0.062667$

$\begin{array}{rr}-0.02748 & 0.009444\end{array}$

$\begin{array}{ll}-0.04593 & -0.009\end{array}$

$-0.07756$

$-0.096$

Alpha $=0.05$

Comparisons for each pair using Student's t

$$
t
$$

1.98862

Abs(Dif)-LSD Middendorf Blck Creek

Middendorf

$-0.07398-0.03611$

$-0.03611-0.09686$

-0.03611
-0.00125

$-0.06178$

Pee Dee

$-0.00125$

Pee Dee

$-0.06178$

McBean

Congaree

Tobacco Rd

$-0.06002$

$-0.0695$

$-0.10052$

$-0.02261$

$-0.08201$

0.011649

$-0.04931$

$-0.03444$

$-0.10168$

$-0.08811$
McBean Congaree
$-0.06002$
Tobacco Rd
$-0.02261$
$-0.08201$
$-0.10168$
$-0.03444$
$-0.03444$
$\quad-0.09358$
$-0.05483$
$-0.12081$
- -0.05483
-0.12081
-0.08961

Dry Branch

0.011649

$-0.04931$

$-0.08811$

$-0.02191$

$-0.08961$

$-0.09358$ 
FIGURE $13 \mathrm{~b}$.

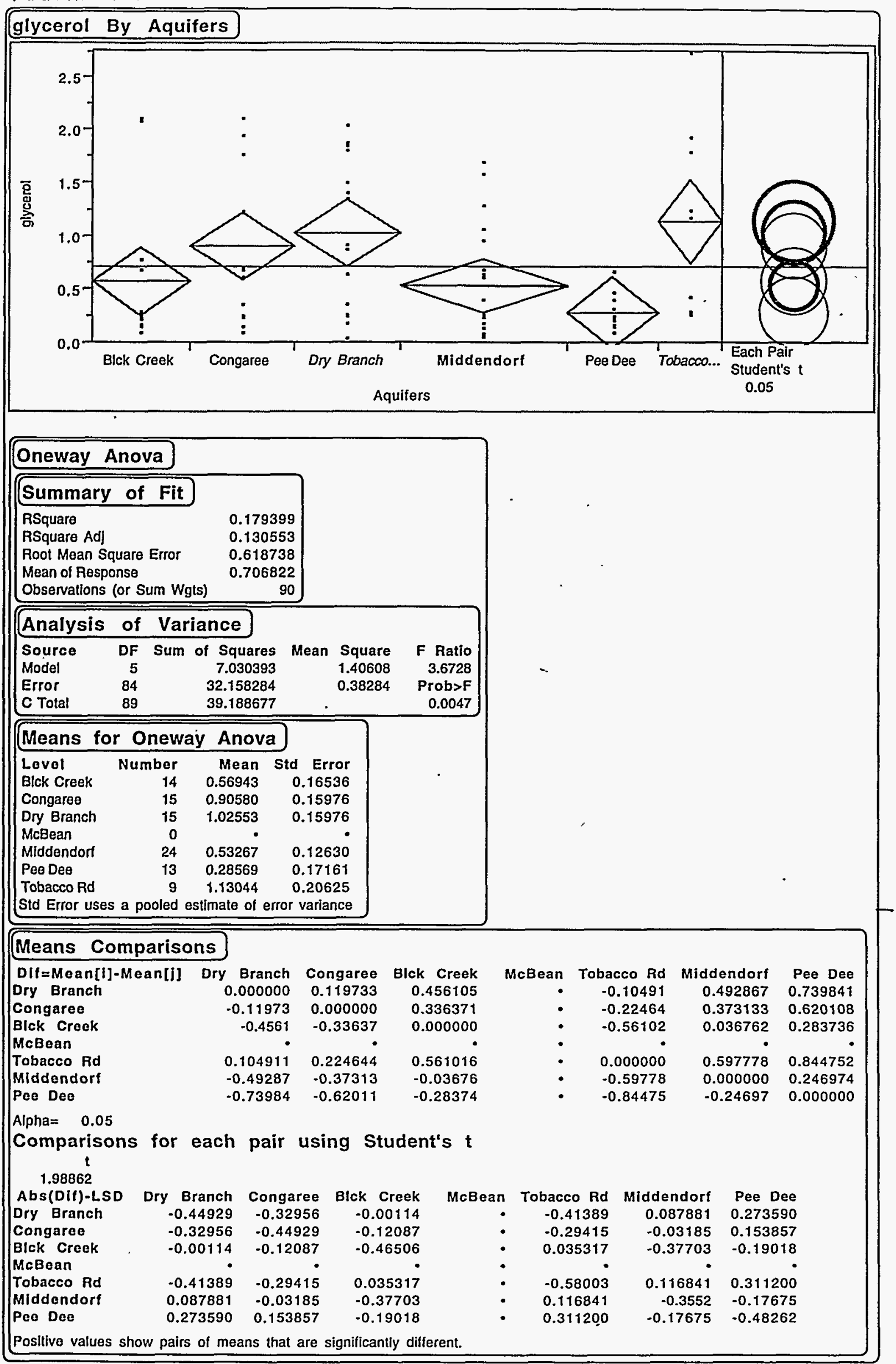


FIGURE 14.

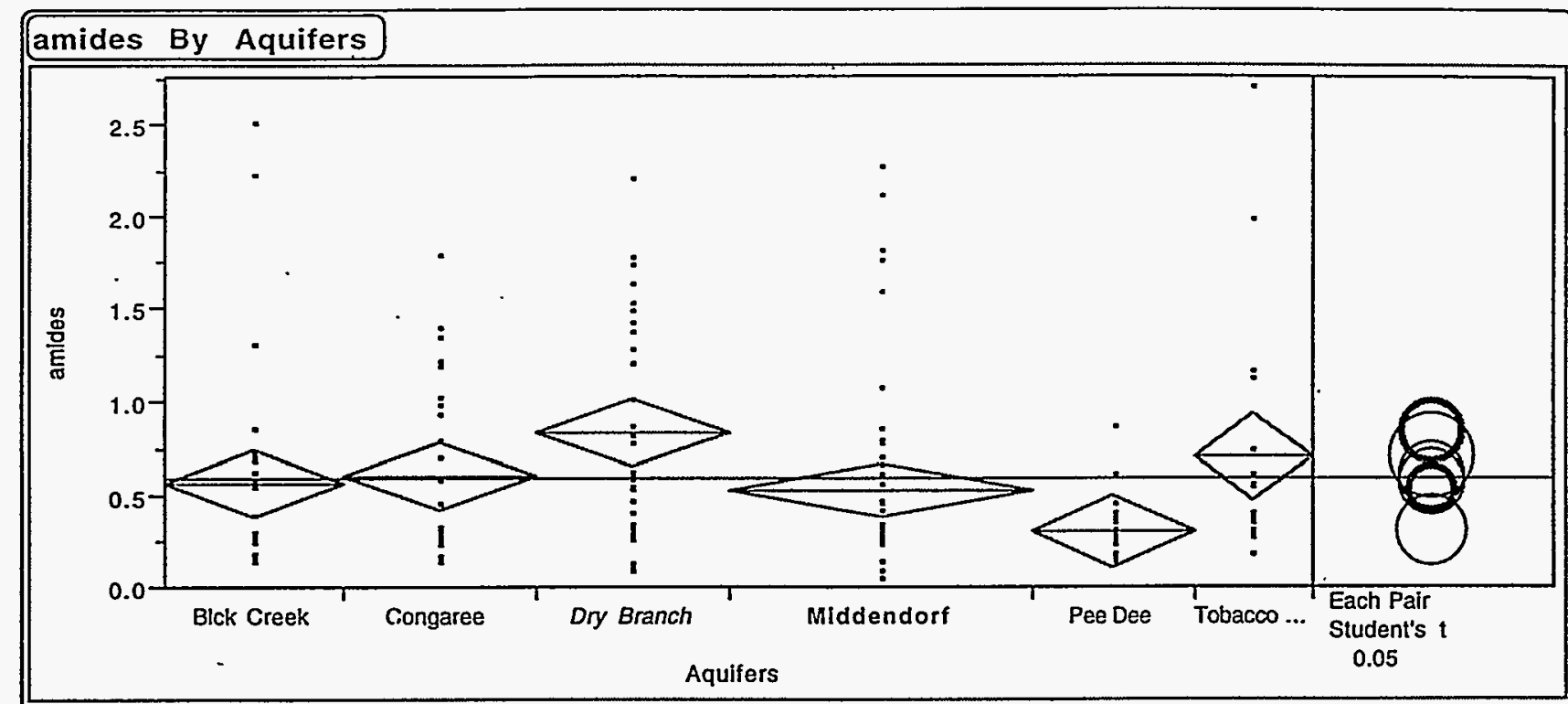

\section{Oneway Anova}

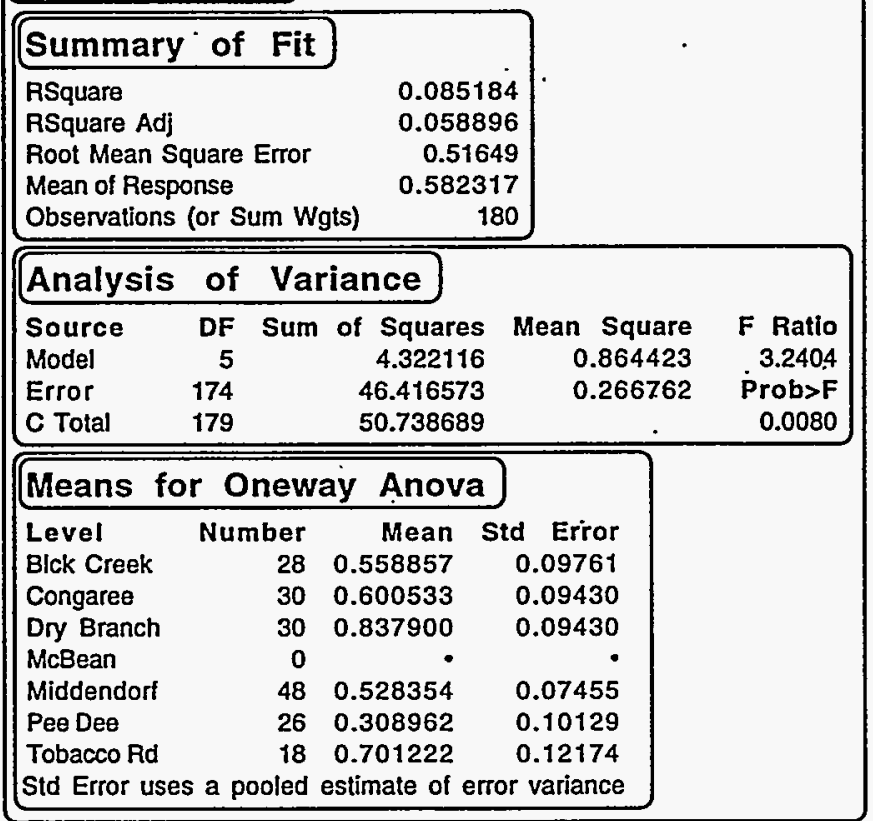

\section{Means Comparisons}

Dif-Mean[i]-Mean[i]

Dry Branch

Congaree

Blck Creek

McBean

Tobacco Rd

Middendorf

Pee Dee

Alpha $=0.05$

Comparisons for each pair using Student's t

$\begin{array}{rrr}0.000000 & 0.237367 & 0.279043 \\ -0.23737 & 0.000000 & 0.041676 \\ -0.27904 & -0.04168 & 0.000000 \\ -0.13668 & 0.100689 & 0.142365 \\ -0.30955 & -0.07218 & -0.0305 \\ -0.52894 & -0.29157 & -0.2499\end{array}$

ongare

- $\quad 0.136678$

- $\quad-0.10069$

- $\quad 0.14237$

-

- 0.000000

- $\quad-0.17287$

$-0.39226$

$\begin{array}{rr}\text { Middendorf } & \text { Pee Dee } \\ 0.309546 & 0.528938 \\ 0.072179 & 0.291572 \\ 0.030503 & 0.249896 \\ 0 & \\ 0.172868 & 0.392261 \\ 0.000000 & 0.219393 \\ -0.21939 & 0.000000\end{array}$

1.97371

Abs(Dif)-LSD

Dry Branch

Congaree

Blck Creek

McBean

Tobacco Rd

Middendort

Pee Dee
Dry Branch $-0.26321$

$-0.02584$

0.011175

$-0.16725$

0.072293

0.255794
Congaree

$-0.02584$

$-0.26321$

$-0.22619$

$-0.20324$

$-0.16507$

0.018427
Blck Creek 0.011175

$-0.22619$

$-0.27245$

$-0.16561$

$-0.21191$

$-0.02774$
McBean

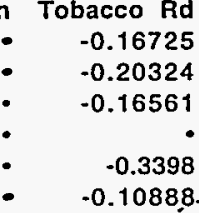

Middendorf 0.072293

$-0.16507$

$-0.21191$

$-0.10888$

$-0.20808$

$-0.02884$
Pee Dee

0.255794

0.018427

$-0.02774$

0.079689

$-0.02884$

$-0.28273$

Positive values show pairs of means that are significantly different. 
FIGURE $14 \mathrm{a}$.

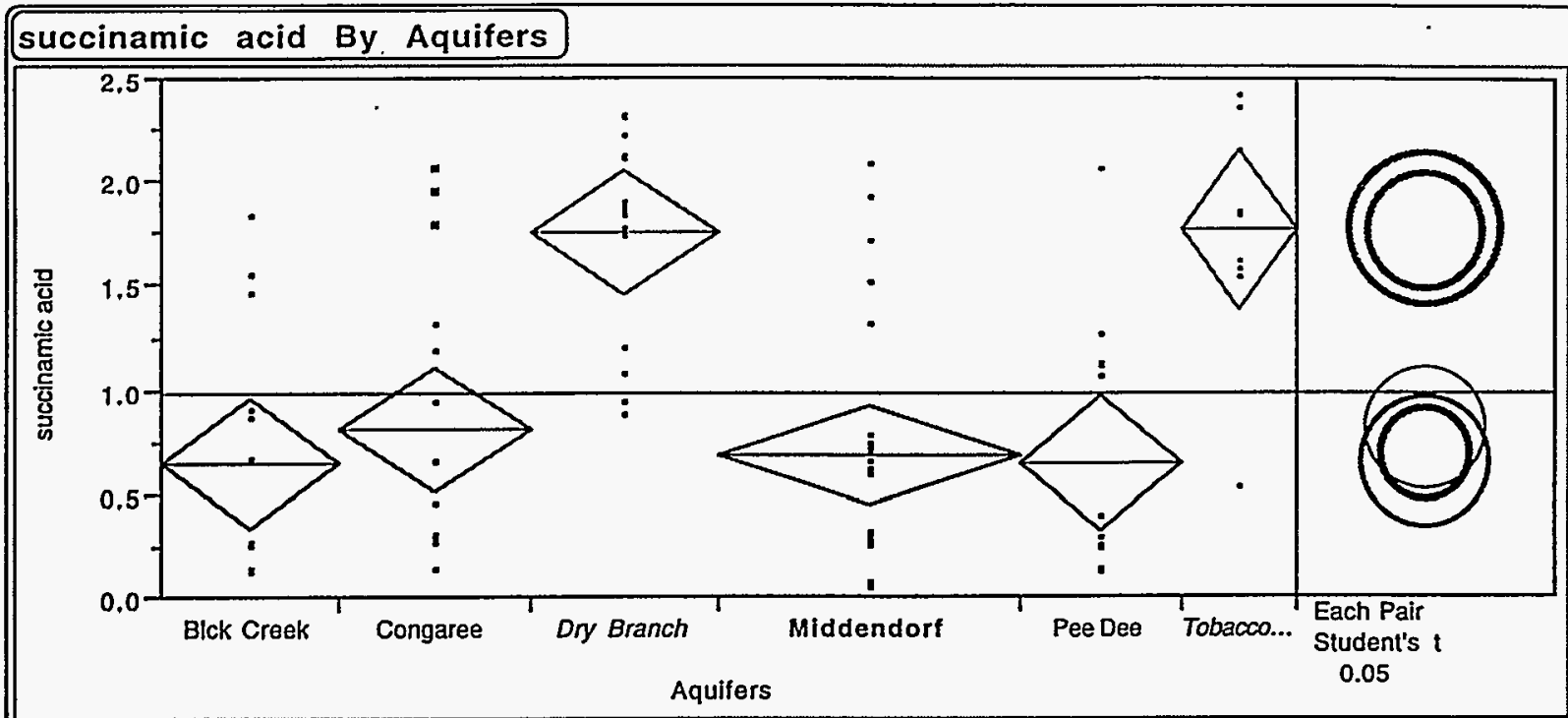

\section{Oneway Anova}

\section{Summary of Fit}

RSquare

RSquare Ad]

Root Mean Square Error

Mean of Response

0.366747

0.596712

Observations (or Sum Wgts)

0.979467

\begin{tabular}{|lrrrrr|}
\hline Analysis & of & \multicolumn{2}{c}{ Variance } & & \\
Source & DF & Sum of Squares & Mean Square & F Ratio \\
Model & 5 & 20.133429 & 4.02669 & 11.3088 \\
Error & 84 & 29.909525 & 0.35607 & Prob>F \\
C Total & 89 & 50.042954 & & 0.0000 \\
\hline
\end{tabular}

Means for Oneway Anova

Level Number ${ }^{\circ}$ Mean Std Error

$\begin{array}{llll}\text { Blck Creek } & 14 & 0.64686 & 0.15948\end{array}$

$\begin{array}{llll}\text { Congaree } & 15 & 0.80413 & 0.15407\end{array}$

$\begin{array}{lrrr}\text { Dry Branch } & 15 & 1.75140 & 0.15407\end{array}$

McBean

Middendorf

Pee Dee

$\begin{array}{rr}0 & \text {. } \\ 24 & 0.68463\end{array}$

.

0.12180

Tobacco Rd

$\begin{array}{rr}13 & 0.64523 \\ 9 & 1.77156\end{array}$

0.16550

0.19890

Std Error uses a pooled estimate of error variance

\section{Means Comparisons}

\begin{tabular}{|c|c|c|c|c|c|c|c|c|}
\hline $\begin{array}{l}\text { Dlf=Mean[1]-Mean[]] } \\
\text { Dry Branch }\end{array}$ & Dry & $\begin{array}{l}\text { Branch } \\
0.00000\end{array}$ & $\begin{array}{r}\text { Congaree } \\
0.94727\end{array}$ & $\begin{array}{r}\text { Blck Creek } \\
1.10454\end{array}$ & $\begin{array}{r}\text { McBean } \\
\text {. }\end{array}$ & $\begin{array}{r}\text { Tobacco Rd } \\
-0.02016\end{array}$ & $\begin{array}{r}\text { Middendorf } \\
1.06678\end{array}$ & $\begin{array}{r}\text { Pee Dee } \\
1.10617\end{array}$ \\
\hline Congaree & & -0.94727 & 0.00000 & 0.15728 & - & -0.96742 & 0.11951 & 0.15890 \\
\hline Blck Creek & & -1.10454 & -0.15728 & 0.00000 & - & -1.12470 & -0.03777 & 0.00163 \\
\hline McBean & & $\bullet$ & • & $\bullet$ & $\bullet$ & $\bullet$ & - & • \\
\hline Tobacco Rd & & 0.02016 & 0.96742 & 1.12470 & - & 0.00000 & 1.08693 & 1.12632 \\
\hline Middendorf & & -1.06678 & -0.11951 & 0.03777 & - & -1.08693 & 0.00000 & 0.03939 \\
\hline Pee Dee & & -1.10617 & -0.15890 & -0.00163 & - & -1.12632 & -0.03939 & 0.00000 \\
\hline
\end{tabular}

Pee Dee

$-1.10617$

$-0.15890$

$-1.12632$

Comparisons for each pair using Student's t

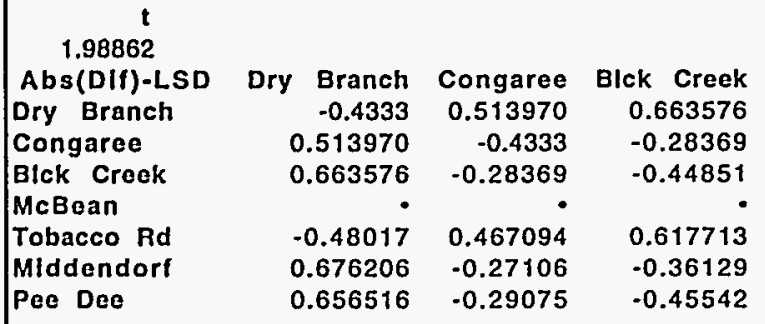

Positive values show pairs of means that are signilicantly different.

\begin{tabular}{rrrr} 
McBean & Tobacco Rd & Middendorf & Pee Dee \\
$\therefore$ & -0.48017 & 0.676206 & 0.656516 \\
$\therefore$ & 0.467094 & -0.27106 & -0.29075 \\
$\therefore$ & 0.617713 & -0.36129 & -0.45542 \\
$\therefore$ & -0.55938 & 0.623114 & 0.611766 \\
\hline & 0.623114 & -0.34255 & -0.36924 \\
- & 0.611766 & -0.36924 & -0.46544
\end{tabular}


FIGURE 14c.

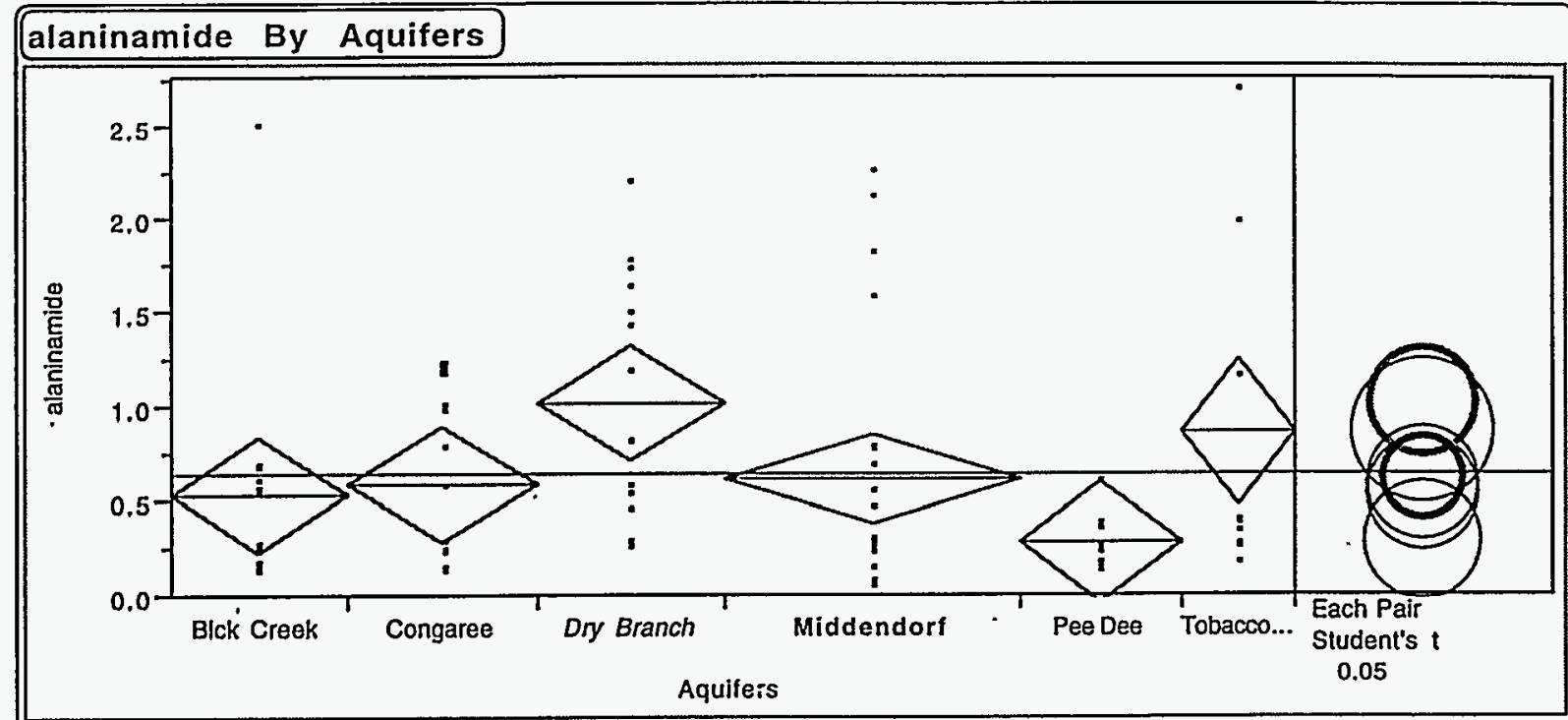

\begin{tabular}{|lr|}
\hline Oneway Anova \\
\hline Summary of Fit \\
\hline RSquare & 0.130632 \\
RSquare Adj & 0.078884 \\
Root Mean Square Error & $\mathbf{0 . 5 9 3 0 3 7}$ \\
Mean of Response & 0.642244 \\
Observattons (or Sum Wgts) & 90 \\
\hline
\end{tabular}

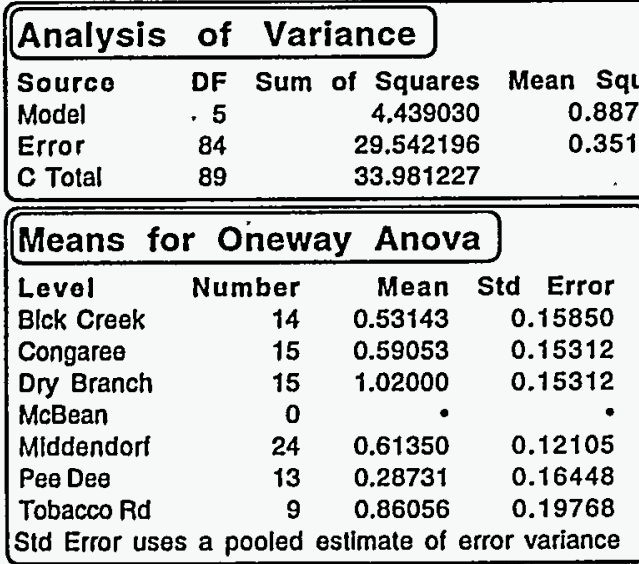

\begin{tabular}{|c|c|c|c|c|c|c|c|}
\hline \multicolumn{8}{|c|}{ Means Comparisons } \\
\hline $\begin{array}{l}\text { Dif=Mean }[1]-\text { Mean }[j] \\
\text { Dry Branch }\end{array}$ & $\begin{array}{r}\text { Dry Branch } \\
0.000000\end{array}$ & $\begin{array}{r}\text { Middendorf } \\
0.406500\end{array}$ & $\begin{array}{r}\text { Blck Creek } \\
0.488571\end{array}$ & McBean & $\begin{array}{r}\text { Tobacco Rd } \\
0.159444\end{array}$ & $\begin{array}{r}\text { Congaree } \\
0.429467\end{array}$ & $\begin{array}{r}\text { Pee. Dee } \\
0.732692\end{array}$ \\
\hline Middendort & -0.4065 & 0.000000 & 0.082071 & - & -0.24706 & 0.022967 & 0.326192 \\
\hline Blck Creek & -0.48857 & -0.08207 & 0.000000 & - & -0.32913 & -0.0591 & 0.244121 \\
\hline McBean & - & - & - & - & . & - & \\
\hline Tobacco Rd & -0.15944 & 0.247056 & 0.329127 & - & 0.000000 & 0.270022 & 0.573248 \\
\hline Congaree & -0.42947 & -0.02297 & 0.059105 & - & -0.27002 & 0.000000 & 0.303226 \\
\hline Pee Dee & -0.73269 & -0.32619 & -0.24412 & - & -0.57325 & -0.30323 & 0.000000 \\
\hline
\end{tabular}

Alpha $=0.05$

Comparisons for each pair using Student's t 1.98862 Abs(DIf)-LSD Dry Branch

$\begin{array}{rrr}\text { Dry Branch } & \text { Middendorf } & \text { Blck Creek } \\ -0.43063 & 0.018337 & 0.050321 \\ 0.018337 & -0.34044 & -0.31453 \\ 0.050321 & -0.31453 & -0.44574 \\ - & - & \\ -0.3378 & -0.2139 & -0.17474 \\ -0.00116 & -0.3652 & -0.37915 \\ 0.285808 & -0.07993 & -0.21011\end{array}$

\begin{tabular}{rr} 
McBean & Tobacco Rd \\
\hline & -0.3378 \\
\hline & -0.2139 \\
\hline & -0.17474 \\
\hline & -0.55594 \\
\hline & -0.22722 \\
\hline & 0.061859
\end{tabular}

$\begin{array}{rr}\text { Congaree } & \text { Pee Dee } \\ -0.00116 & 0.285808 \\ -0.3652 & -0.07993 \\ -0.37915 & -0.21011 \\ - & \\ -0.22722 & 0.061859 \\ -0.43063 & -0.14366 \\ -0.14366 & -0.46257\end{array}$
Mlddendor Blck Creek McBean Tobacco Rd Congaree 0.07993

$-0.21011$

Positive values show pairs of means that are significantly different 
EIGURE 15.

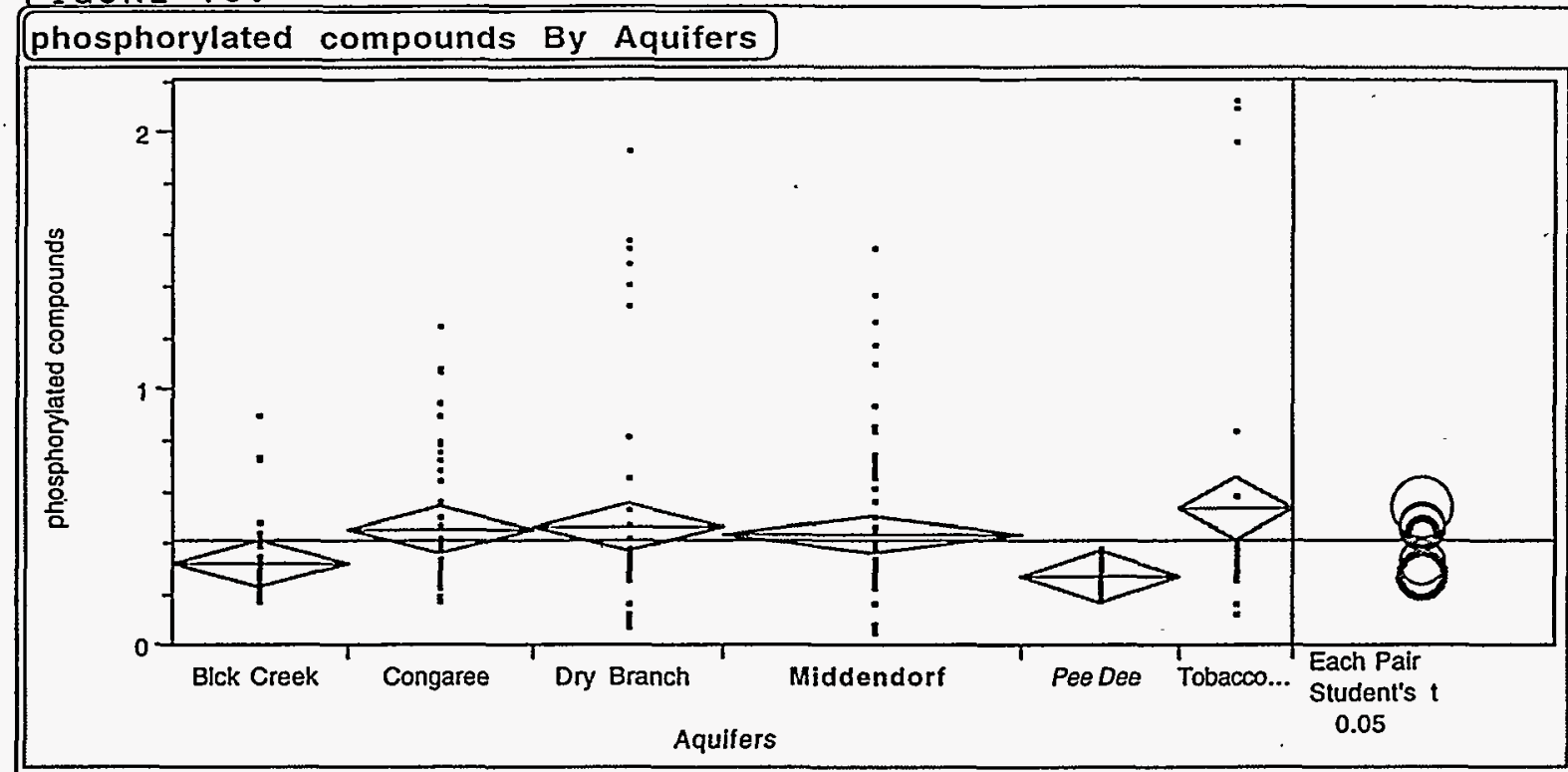

\section{Oneway Anova}

\section{Summary of Fit}

RSquare

RSquare Adj

Root Mean Square Error

Mean of Response

Observations (or Sum Wgts)

0.037289

0,329997

0.409626

270

Analysis of Variance

Source DF Sum of Squares Mean Square F Ratio

$\begin{array}{lrrrr}\text { Model } & 5 & 1.679127 & 0.335825 & 3.0838\end{array}$

$\begin{array}{lllll}\text { Error } & 264 & 28.749108 & 0.108898 & \text { Prob }>F\end{array}$

\begin{tabular}{lll}
$C$ Total. & 269 & 30.428235 \\
\hline
\end{tabular}

0.0101

Means for Oneway Anova.

Level Number Mean Std Error

Blck Creek $\quad 420.321762 \cdot 0.05092$

$\begin{array}{llll}\text { Congaree } & 45 & 0.454578 & 0.04919\end{array}$

$\begin{array}{lrll}\text { Dry Branch } & 45 & 0.463356 & 0.04919\end{array}$

McBean

Middendor

Pee Dee

$72 \quad 0.427750$

$39 \quad 0.273718$

Tobacco Rd

$27 \quad 0.529815$

0.03889

0.05284

0.06351

Std Error uses a pooled estimảte of error variance

\begin{tabular}{|lrrrrrrrr}
\hline Means Comparisons & & & & & \\
\hline
\end{tabular}

Alpha $=0.05$

Comparisons for each pair using Student's $t$

$\mathbf{t}$

1.96902

Abs(Dif)-LSD

Dry Branch

Congaree

Blck Creek

McBean

Tobacco Rd

Middendorf

Pee Dee
McBean Tobacco Rd Middendorf

$-0.09172$

$-0.08294$

0.047774

$-0.17684$

$-0.04457$

0.093423 $\begin{array}{rr}\text { iddendorf } & \text { Pee Dee } \\ -0.08787 & 0.047483 \\ -0.09665 & 0.038705 \\ -0.02017 & -0.09645 \\ -0.04457 & 0.093423 \\ -0.10829 & 0.024844 \\ 0.024844 & -0.14714\end{array}$

Positive values show pairs of means that are significantly different 
FIGURÉ $15 \mathrm{a}$.

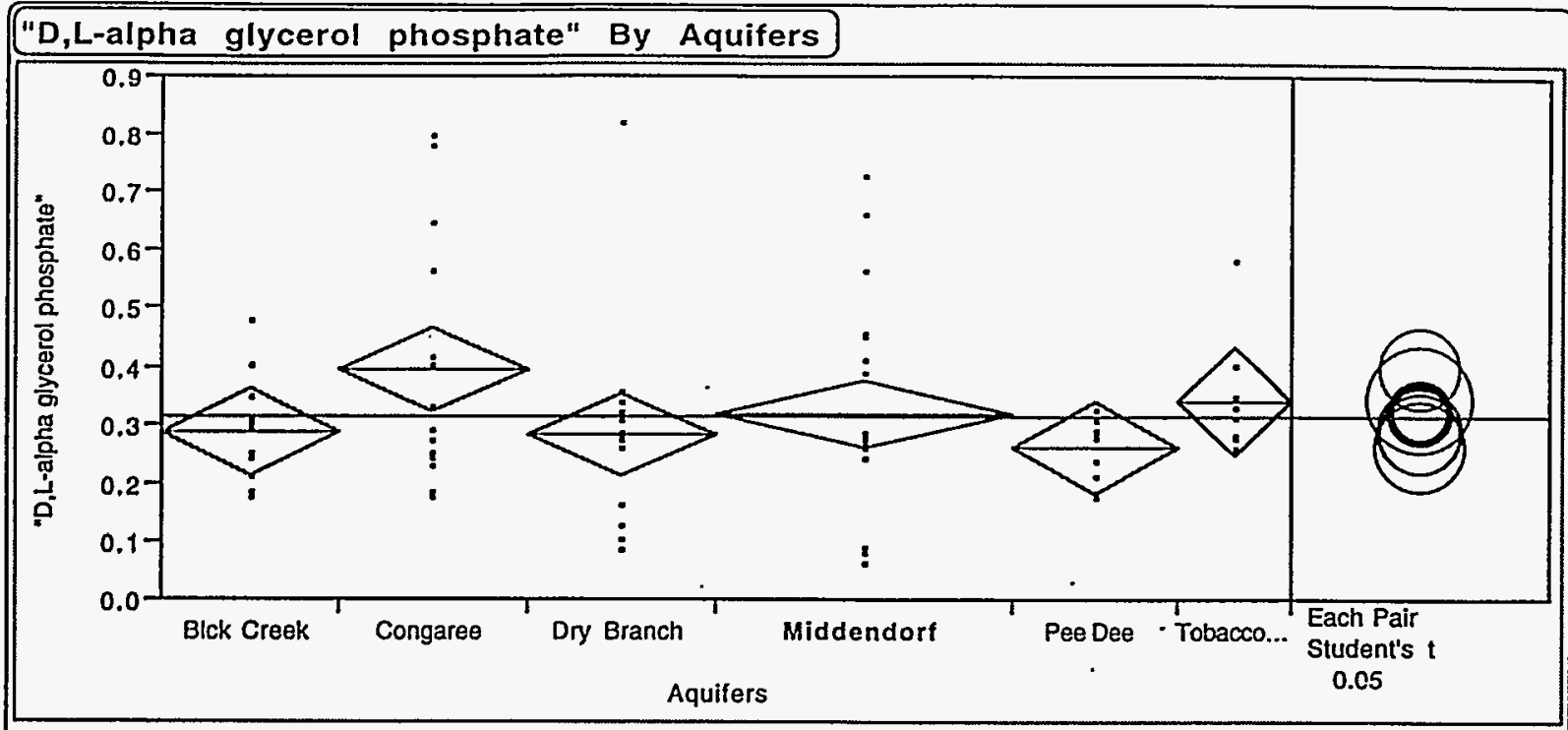

\section{Oneway. Anova}

Summary of Fit

RSquare Adj

Root Mean Square Error

Mean of Response

Observations (or Sum Wgts)

$$
\begin{aligned}
& 0.084259 \\
& 0.029751 \\
& 0.147122 \\
& 0.315078
\end{aligned}
$$

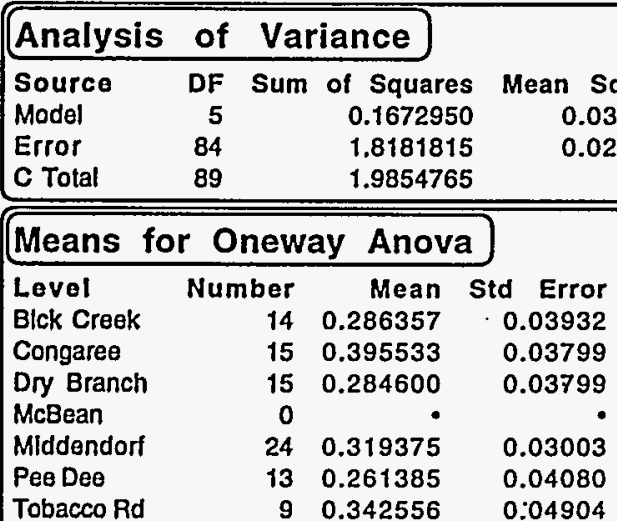

Sid Error uses a pooled estimate of error variance

\section{Means Comparisons}

Dif=Moan[i]-Mean[j] Congaree

Congaroo

Blck Creok

Dry Branch

McBean

Tobacco Rd

Middendorf

Pee Dee

Alpha $=0.05$

Comparisons for each pair using Student's $t$ $t$

1,98862

Abs(DII)-LSD

Congareo

Blck Creek

Dry Branch

McBean

Tobacco Rd

Mlddendorf

Peo Dee

Congaree
-0.10683
0.000454
0.004102
$\cdot$
-0.07038
-0.02014
0.023284

lck Creek

0.000454

$-0.11058$

$-0.10697$

Creek
0.109176
0.000000
-0.00176
.
0.056198
0.033018
-0.02497

Dry Branch 0.110933 0.001757 0.000000

0.057956

0.034775

$-0.02322$
$-0.06537$

$-0.08772$
$-0.0688$
Dry Branch

0.004102

$-0.10697$

$-0.10683$

$-0.0654$

$-0.06152$

$-0.08765$
McBean Tobacco Rd $-0.07038$

$-0.0688$

$-0.0654$

$-0.13792$

$-0.09118$

$-0.0457$
Middendorf $-0.02014$

$-0.06537$

$-0.06152$

$-0.09118$

$-0.08446$

$-0.04276$
Pee Dee

0.023284

$-0.08772$

$-0.08765$

$-0.0457$

$-0.04276$

$-0.11476$

Positlve values show pairs of means that are significantly different. 
FIGURE 15b.

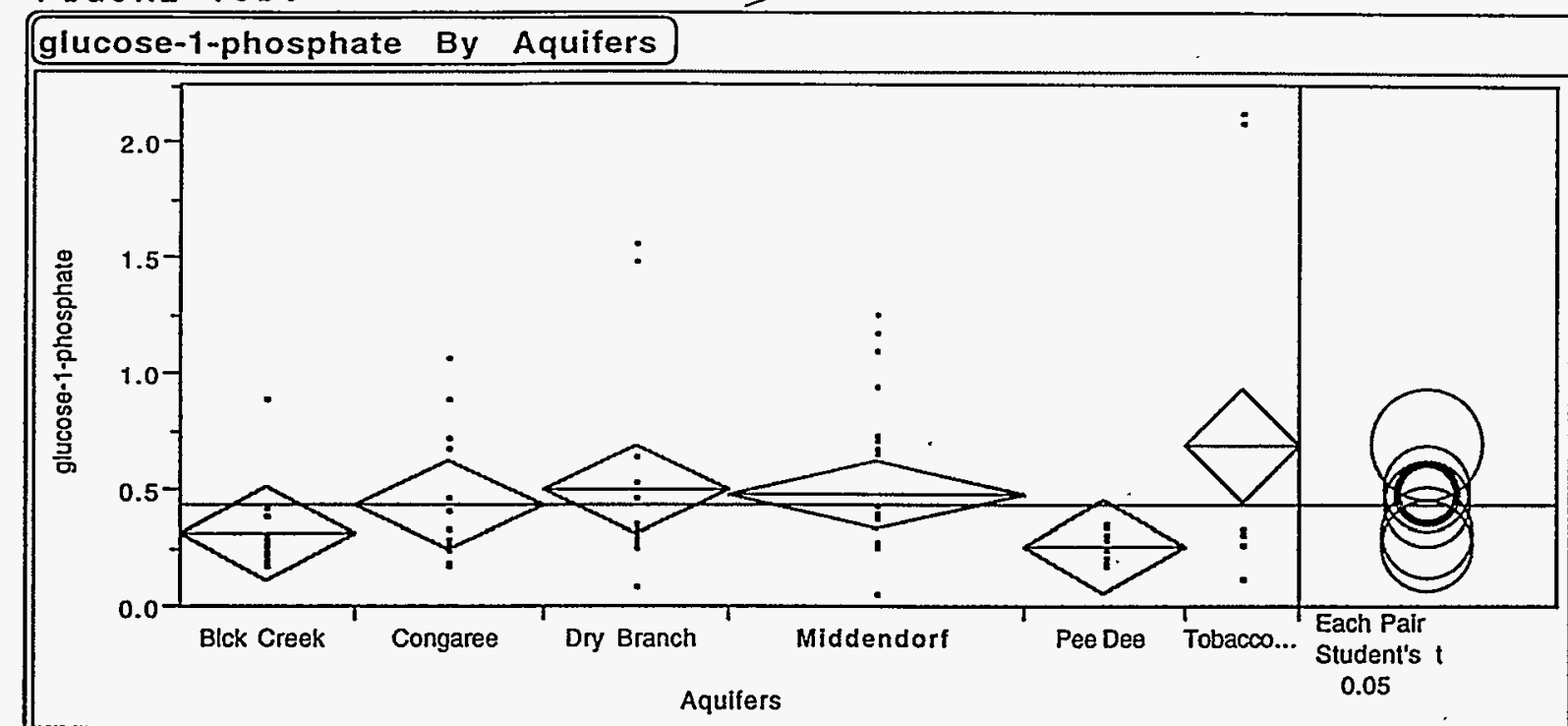

\section{Oneway Anova}

\begin{tabular}{|lr|}
\hline Summary of Fit & \\
RSquare & 0.095217 \\
RSquare Adj & 0.041361 \\
Root Mean Square Error & 0.380073 \\
Mean of Response & 0.443467 \\
Observations (or Sum Wgts) & 90 \\
\hline
\end{tabular}

\begin{tabular}{|lrrrr|}
\hline Analysis & of & Variance & & \\
Source & DF & Sum of Squares & Mean Square & F Ratio \\
Model & 5 & 1.276981 & 0.255396 & 1.7680 \\
Error & 84 & 12.134267 & 0.144456 & Prob $>$ F \\
C Total & 89 & 13.411248 & & 0.1283 \\
\hline
\end{tabular}

Means for Oneway Anova

Level Number Mean Std Error

$\begin{array}{lrrr}\text { Blck Creek } & 14 & 0.319429 & 0.10158\end{array}$

$\begin{array}{lll}15 & 0.443667\end{array}$

$\begin{array}{llll}\text { Dry Branch } & 15 & 0.501867 & 0.09813\end{array}$

McBean

Middendorf

Pee Dee

Tobacco Rd

$240.483333 \quad 0.07758$

$13 \quad 0.264077 \quad 0.10541$

Std Error uses a pooled estimate of error variance

Means Comparisons

Dif=Mean[i]-Mean[j] Dry Branch

Dry Branch

Middendorf

Blck Creek

McBean

Tobacco Rd

Congaree

Pee Dee

Alpha $=0.05$

Comparisons for each pair using Student's t

1.98862

Abs(DIf)-LSD

Dry Branch

Middendorf

Blck Creek

McBean

Tobacco Rd

Congaree

Pee Dee

0.000000

Middendorf Blck Creek

0.018533

0.182438

$-0.01853$

$-0.18244$

0.000000

0.163905

0.000000

0.189689

$-0.0582$

$-0.23779$
0.208222

$-0.03967$

0.372127

0.124238

$-0.05535$
McBean Tobacco Rd Congaree

$-0.18969$

$-0.20822$

$-0.37213$

0.000000

$-0.24789$

$-0.42748$
0.058200

0.039667

$-0.12424$

0.247889

0.000000

$-0.17959$
Pee Dee

0.237790

0.219256

0.055352

0.427479

0.179590

0.000000

Positive values show pairs of means that are significantly different.

McBea

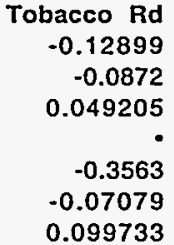

Congaree
-0.21779
-0.2091
-0.15663
-0.07079
-0.27599
-0.10681

Pee Dee

$-0.04861$

$-0.04102$

$-0.23576$

0.099733

$-0.10681$

$-0.29646$ 
FIGURE $15 \mathrm{c}$.

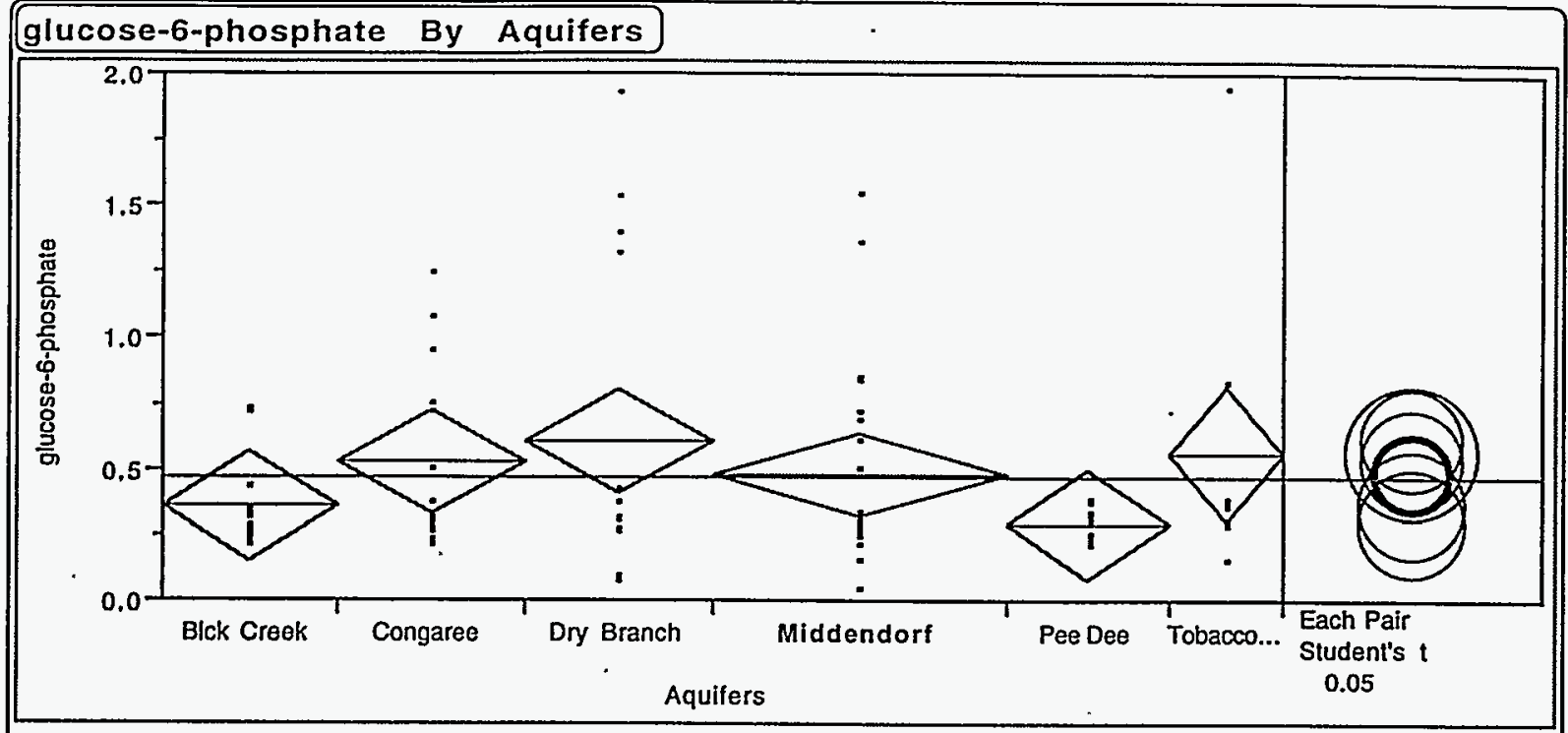

\section{Oneway Anova}

\section{Summary of Fit}

RSquare

RSquare Ad]

Root Mean Square Error

Mean of Respionse

0.068623

0.391057

Observations (or Sum Wgts)

Analysis of Variance

\begin{tabular}{|lrrrrr} 
Source & DF & Sum of Squares & Mean Square & F Ratio \\
Model & 5 & 0.946460 & 0.189292 & 1.2378 \\
Error & 84 & 12.845756 & 0.152926 & Prob>F \\
C Total & 89 & 13.792216 &. & 0.2988 \\
\hline
\end{tabular}

Means for Oneway. Anova

Level

Blck Creek

Congaree

Dry Branch

McBean

Middendorf

Pee Dee

Tobacco Rd

Number

Mean Std Error

140.359500

- 150.524533

0.10451
0.10097

150.603600

0

240.480542

$13 \quad 0.295692$

0.10097

Std Error uses a pooled estimate of error variance

Means Comparisons

Dif=Moan[I]-Mean[i] Dry Branch

Dry Branch

Congaree

Blck Creek

McBean

Tobacco Rd

Middendorf

Pee Dee

0.000000

$-0.07907$

$-0.2441$

$-0.04827$

$-0.12306$

$-0.30791$
Congaree 0.079067

0.000000

$-0.16503$

0.030800

$-0.04399$

$-0.22884$
Blck Creek 0.244100 0.165033 0.000000

0.195833

0.121042

$-0.06381$
McBean Tobacco Rd 0.048267

$-0.0308$

$-0.19583$

0.000000

$-0.07479$

$-0.25964$
Middendorf

Pee Dee

$\begin{array}{ll}0.043992 & 0.228841\end{array}$

$\begin{array}{ll}-0.12104 & 0.063808\end{array}$

0.074792

0.000000

$-0.18485$

Comparisons for each pair using Student's t

1.98862

Abs(DIf)-LSD

Dry Branch

Congaree

Blck Croek

McBean

Tobacco Rd

Middendorf

Poo Deo

$$
\begin{array}{r}
\text { Dry Branch } \\
-0.28396 \\
-0.2049
\end{array}
$$

$-0.04489$

$-0.27962$

$-0.1329$

0.013226
Congaree Blck Creek $: 0.2049$

$-0.28396$

$-0.12396$

$-0.29709$

$-0.21197$

$-0.06584$
$-0.04489$

$-0.29393$

$-0.13642$

.0 .14048

$-0.23572$
McBean Tobacco Rd $-0.27962$

$-0.29709$

$-0.13642$

$-0.36659$

$-0.22917$

$-0.07758$
Middendorf

$\begin{array}{ll}-0.1329 & 0.013226\end{array}$

$-0.21197-0.06584$

$-0.14048 \quad-0.23572$

$\begin{array}{rr}-0.22917-0.07758 \\ -0.02449 & -0.08295\end{array}$

$-0.22449-0.08295$

$-0.08295-0.30502$

Positive values show pairs of means that are significantly different. 
FIGURE 16.

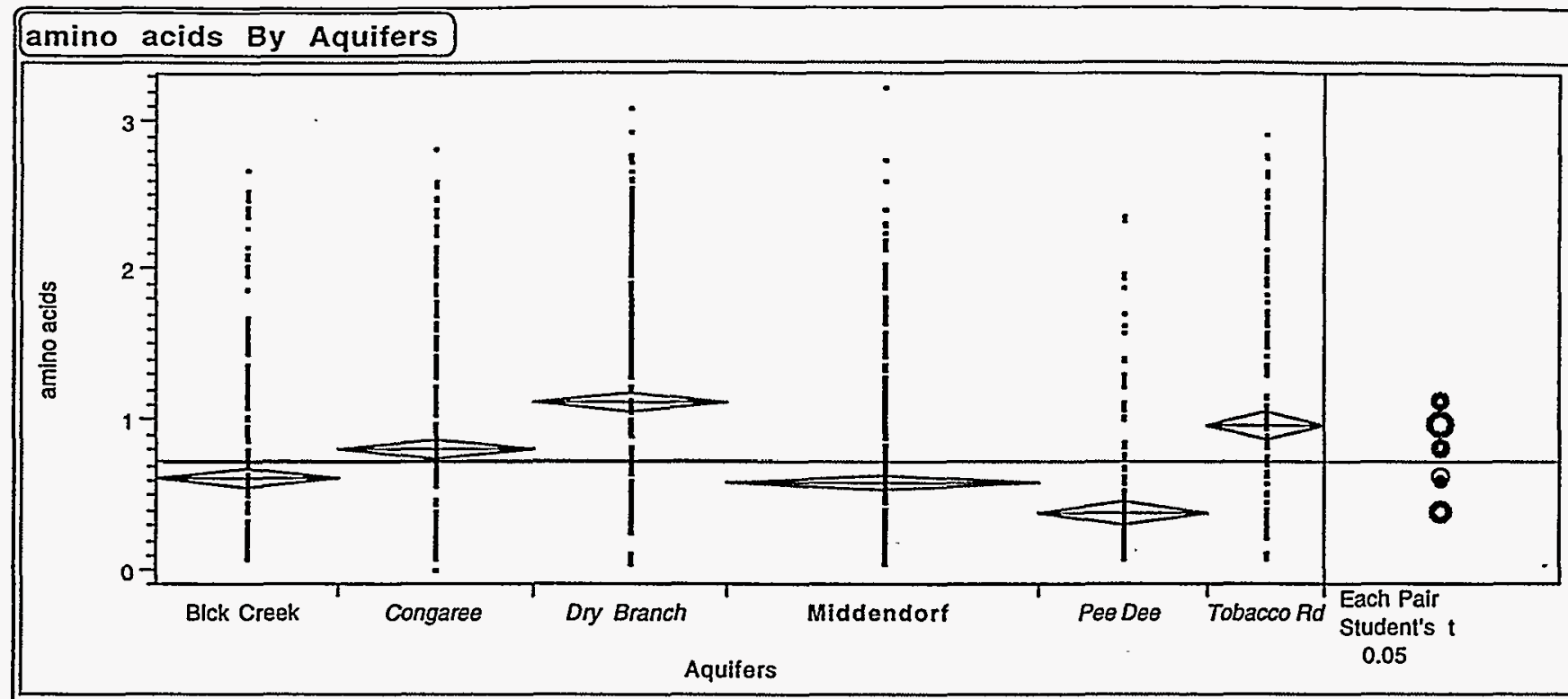

\section{Oneway Anova}

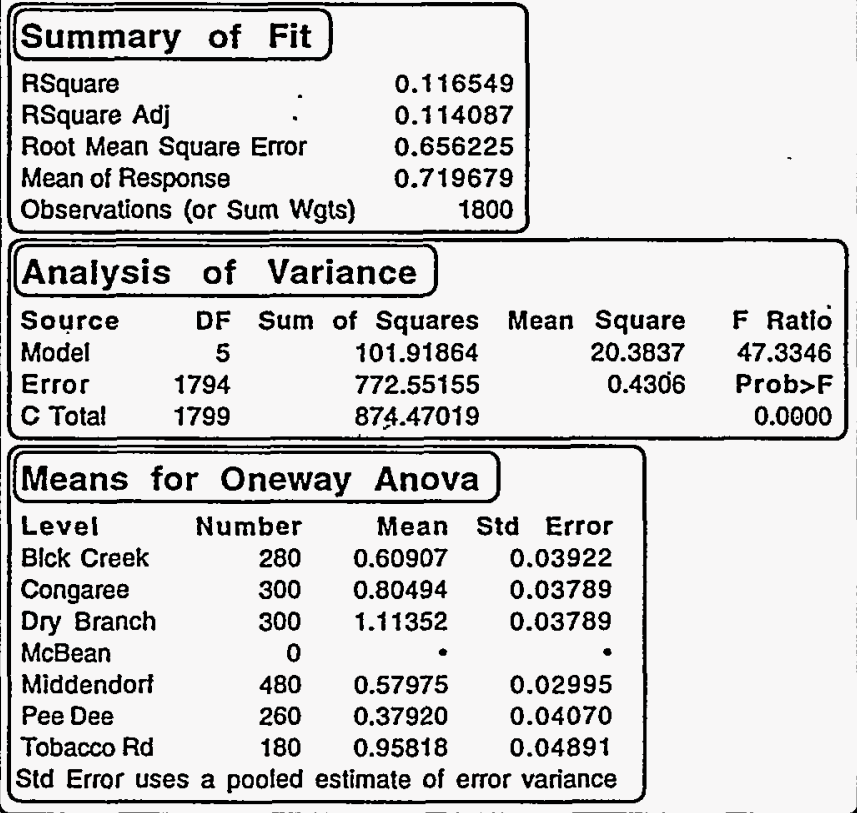

\begin{tabular}{|c|c|c|c|c|c|c|c|}
\hline Means Compariso & & & & & & & \\
\hline Dif=Mean[i]-Mean[i] & Dry Branch & Congaree & Blck Creek & McBean & Tobacco Rd & Middendorf & Pee Dee \\
\hline Dry Branch & 0.000000 & 0.308577 & 0.504452 & - & 0.155337 & 0.533772 & 0.734320 \\
\hline Congaree & -0.30858 & 0.000000 & 0.195875 & - & -0.15324 & 0.225195 & 0.425743 \\
\hline Blck Creek & -0.50445 & -0.19588 & 0.000000 & - & -0.34912 & 0.029320 & 0.229868 \\
\hline McBean & - & - & - & - & - & - & \\
\hline Tobacco Rd & -0.15534 & 0.153240 & 0.349115 & - & 0.000000 & 0.378435 & 0.578983 \\
\hline Middendorf & -0.53377 & -0.2252 & -0.02932 & - & -0.37844 & 0.000000 & 0.200548 \\
\hline Pee Dee & -0.73432 & -0.42574 & -0.22987 & - & -0.57898 & -0.20055 & 0.000000 \\
\hline
\end{tabular}

Alpha $=0.05$

Comparisons for each pair using Student's $t$

1.96132

Abs(Dit)-LSD

Dry Branch

Congaree

Blck Creek

McBean

Tobacco Rd

Middendorf

Dry Branch

Congaree Blck Creek

0.397503

$\begin{array}{lll}0.203488 & -0.10509 & 0.088927\end{array}$

$\begin{array}{lll}0.397503 & 0.088927 & -0.10878\end{array}$

$0.033991 \quad 0.031894$

$0.439046 \quad 0.130470$

0.625264

0.316688

0.226155

$-0.06747$

0.119019

McBean Tobacco Rd 0.033991

0.031894

0.226155

$-0.13567$

0.265945 .

0.454186
Middendorf

$0.130470 \quad 0.316688$

$-0.06747$

0.265945

$\begin{array}{ll}-0.08308 & 0.101440\end{array}$

$0.101440 \quad-0.11288$

Positive values show pairs of means that are significantly different. 
FIGURE $16 \mathrm{a}$.

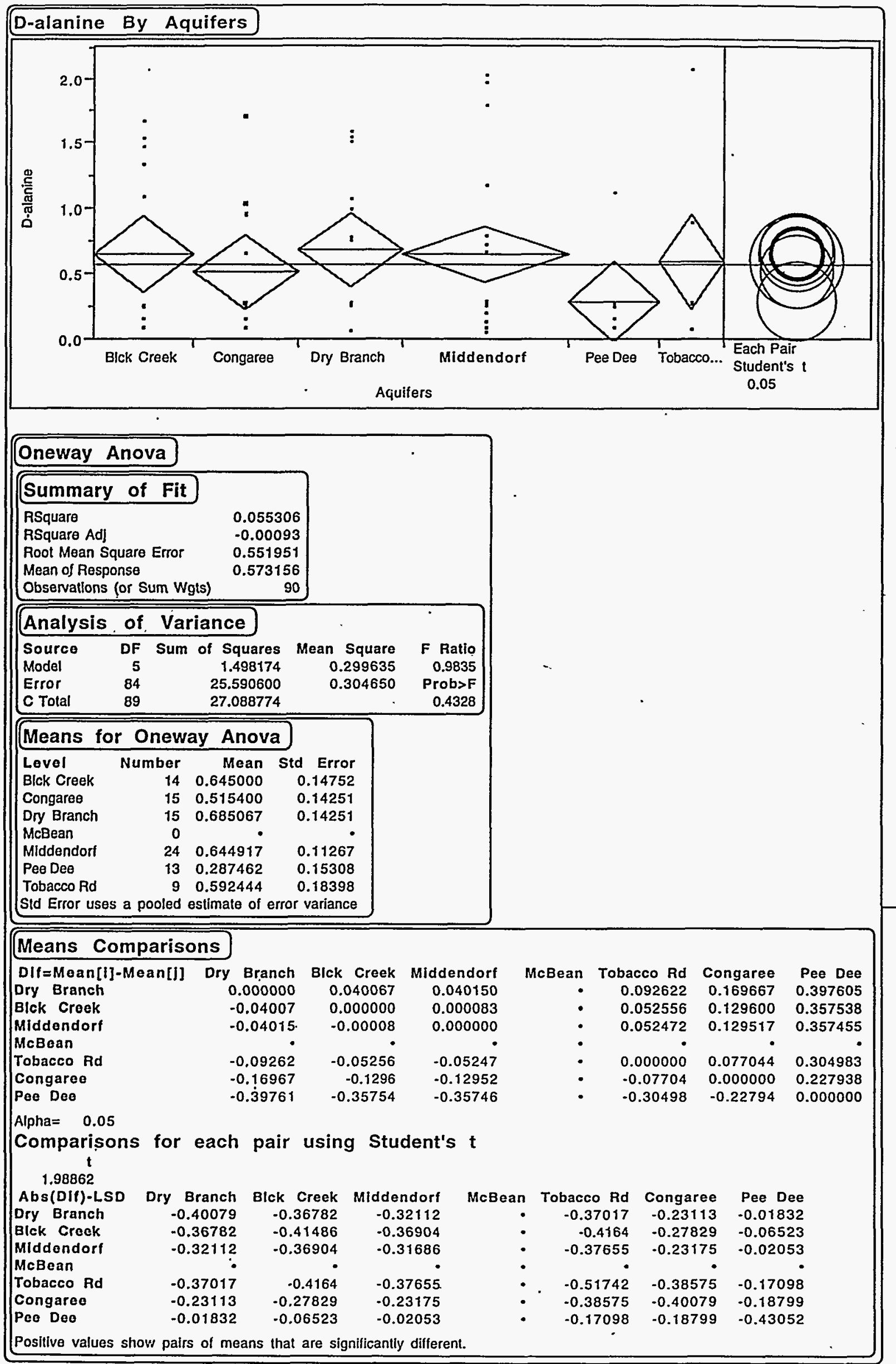




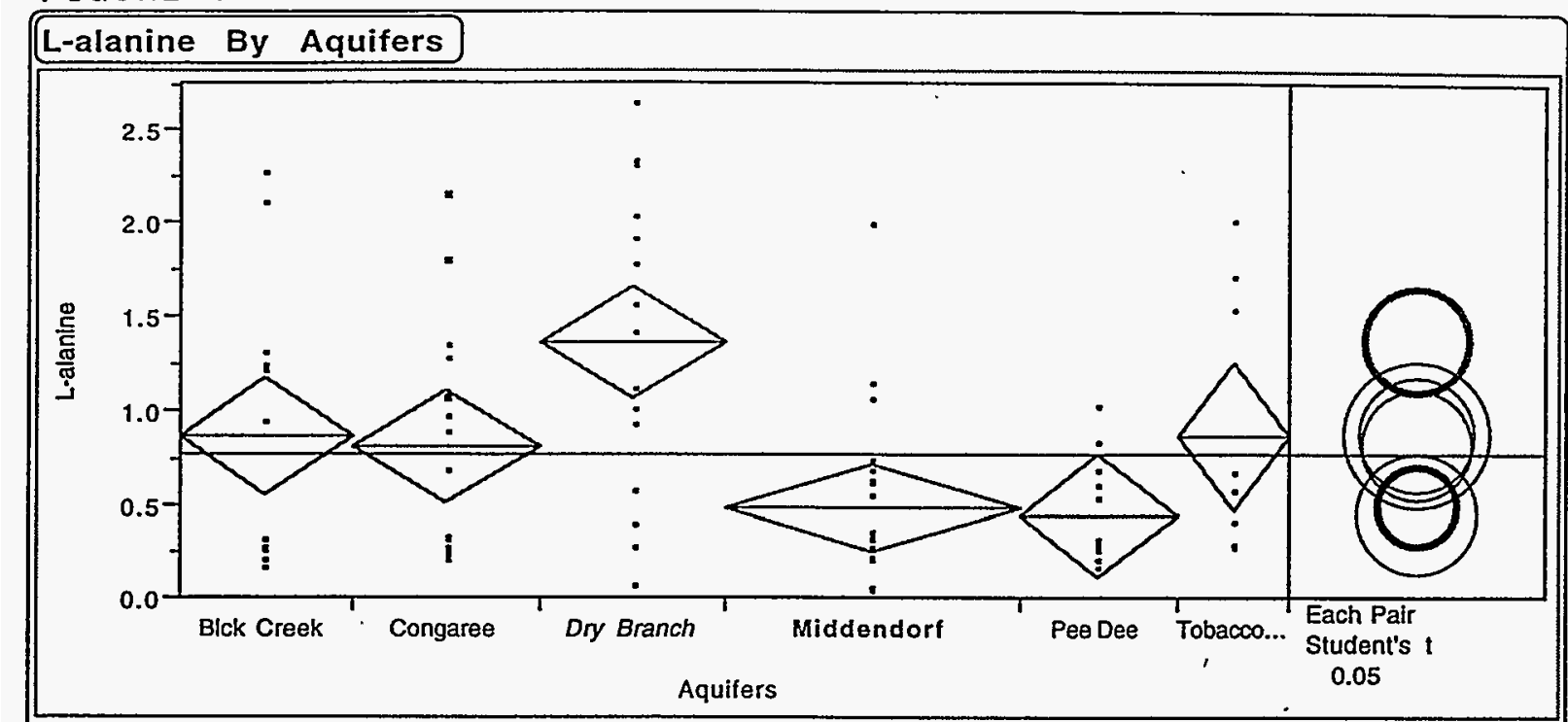

\section{Oneway Anova}

Summary of Fit

RSquare

RSquare Adj

Root Mean Square Error

Mean of Response

Observations (or Sum Wgts)

Analysis . of Variance

\begin{tabular}{|lrrrr|} 
Source & DF & Sum of Squares & Mean Square & F Ratio \\
Model & 5 & 8.875176 & 1.77504 & 4.9200 \\
Error & 84 & 30.305308 & 0.36078 & Prob $>$ F \\
C Total & 89 & 39.180483 & & 0.0005 \\
\hline
\end{tabular}

Means for Oneway Anova

Level Number Mean Std Error

$\begin{array}{llll}\text { Blck Creek } & 14 & 0.86379 & 0.16053\end{array}$

$\begin{array}{llll}\text { Congaree } & 15 & 0.80220 & 0.15509 \\ & 15 & 1.35787 & 0.15509\end{array}$

$\begin{array}{llll}\text { Dry Branch } & 15 & 1.35787 & 0.15509\end{array}$

McBean

$\begin{array}{llll}\text { Middendorf } & 24 & 0.47996 & 0.12261\end{array}$

$\begin{array}{llll}\text { Pee Dee } & 13 & 0.43554 & 0.16659\end{array}$

$\begin{array}{llll}\text { Tobacco Rd } & 9 & 0.86411 & 0.20022\end{array}$

Sid Error uses a pooled estimate of error variance

Means Comparisons

Dif=Mean[i]-Mean[j] Dry Branch

Dry Branch

Blck Creek

Congaree.

McBean

Tobacco Rd

Middendorf

Pee Dee

0.000000

$-0.49408$

$-0.55567$

Blck Creek

Congaree

$0.000000 \quad 0.061586$

$-0.06159$

$-0.49376$

$-0.87791$

$-0.92233$

0.000325

$-0.38383$

$-0.42825$

0.000000

0.061911

$-0.32224$

$-0.36666$

Alpha $=0.05$

Comparisons for each pair using Student's t $t$

1.98862

Abs(Dif)-LSD

Dry Branch

Blck Creek

Congaree

McBean

Tobacco Rd

Middendorf

Pee Dee

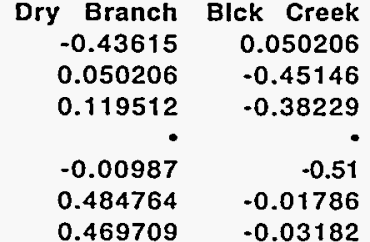

Congaree

0.119512

$-0.38229$

$-0.43615$

$-0.44172$

$-0.0709$

$-0.08596$
McBean Tobacco Rd $-0.00987$ $-0.51$ $-0.44172$

$-0.56307$

$-0.08272$

$-0.08938$
Middendorf 0.484764 $-0.01786$

$-0.0709$

$-0.08272$

$-0.34481$

$-0.36691$
Pee Dee 0.469709

$-0.03182$

$-0.08596$

$-0.08938$

$-0.36691$

$-0.46851$

Positive values show pairs of means that are significantly different. 
FIGURE $16 \mathrm{c}$.

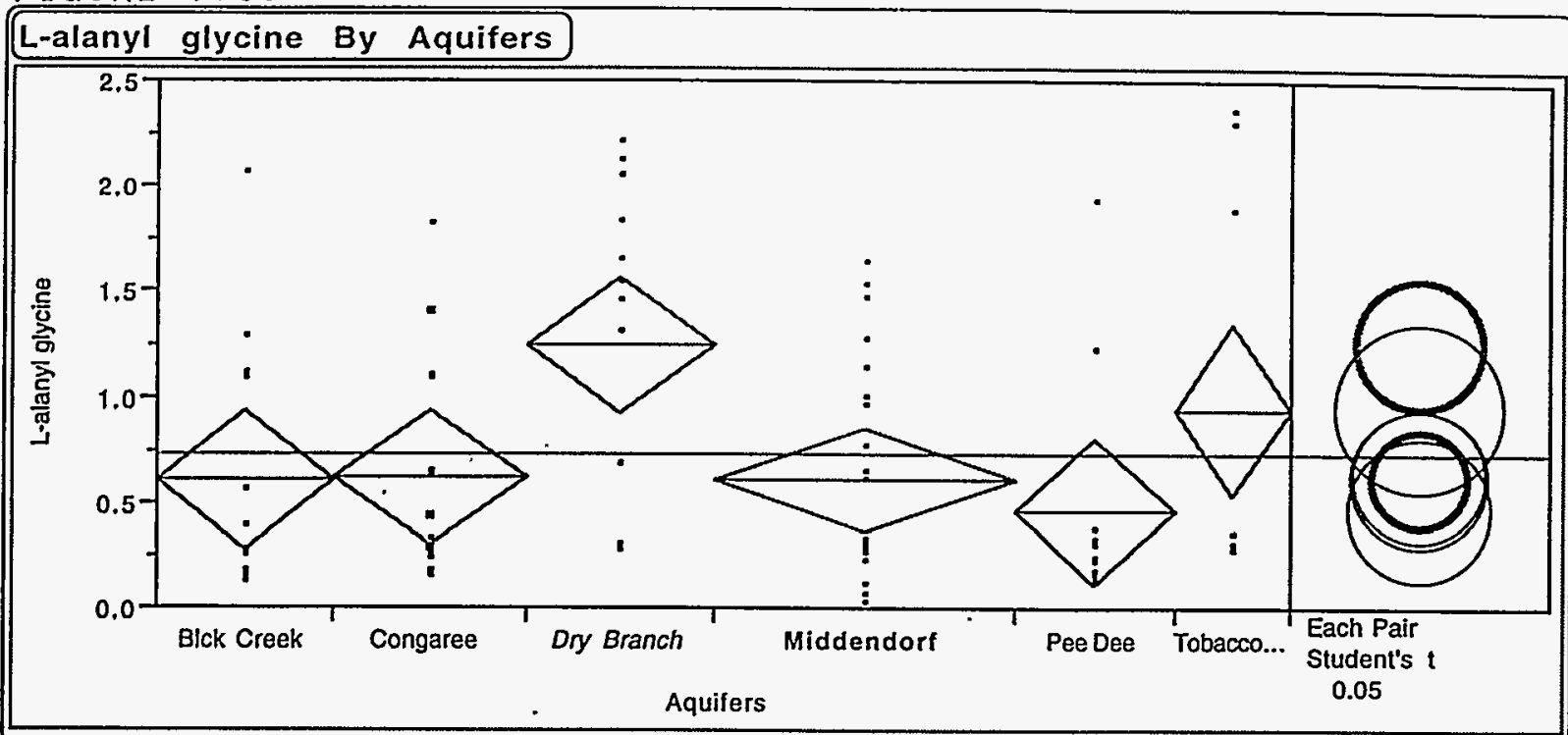

\section{Oneway Anova}

\section{Summary of Fit}

RSquare

RSquare Adj

Root Mean Square Error

Mean of Response

Observalions (or Sum Wgts)

0.155463

0.105193

0.624334

0.726944

\begin{tabular}{|lrrrrr|}
\hline Analysis & of & Variance & & \\
Source & DF & Sum of Squares & Mean Square & F Ratio \\
Model & 5 & 6.027282 & 1.20546 & 3.0926 \\
Error & 84 & 32.742641 & 0.38979 & Prob $>F$ \\
C Total & 89 & 38.769923 & & 0.0130 \\
\hline
\end{tabular}

Means for Oneway Anova

Level Number Mean Std Error

$\begin{array}{lrrr}\text { Blck Creek } & 14 & 0.60329 & 0.16686 \\ \text { Congare } & 15 & 0.61787 & 0.16120\end{array}$

$\begin{array}{llll}\text { Congaree } & 15 & 0.61787 & 0.16120\end{array}$

$\begin{array}{lrrr}\text { Dry Branch } & 15 & 1.24200 & 0.16120\end{array}$

McBean

Mlddendorf

Pee Dee

Tobacco Rd

0.61087

0.45954

$0.93844^{\circ}$

0.12744

0.17316

Std Error uses a pooled estimate of error variance

\section{Means Comparisons}

Dlf=Mean[I]-Mean[J] Dry Branch Congaree Blck Creek

Dry Branch

0.000000

0.624133

\subsection{4}

Congaree

$-0.62413$

0.000000

0.014581

Blck Creok

$-0.63871$

$-0.0145,8$

Tobaceo Rd

Mlddendort

$\begin{array}{ll}-0.30356 & 0.320578\end{array}$

$-0.63113-0.00699$

Pee Dee

$-0.78246$

$-0.15833$

0.000000

0.335159

0.007589

$-0.14375$

McBean Tobacco Rd Middendorf

0.303556

$-0.32058$

$-0.33516$

0.000000

$-0.32757$

$-0.47891$
0.631125 Pee Dee

$0.006992 \quad 0.158328$

$\begin{array}{ll}-0.00759 & 0.143747\end{array}$

0.327569

0.478906

$0.000000 \quad 0.151337$

$\begin{array}{ll}-0.15134 & 0.000000\end{array}$

Alpha $=0.05$

Comparisons for each pair using Student's $t$ $\mathbf{t}$

1,98862

Abs(DIf)-LS

Dry Branch

Congaree

Blck Creek

McBean

Tobacco Rd

Middendorf

Peo Dee

Positive values show pairs of means that are significantly different.

$\begin{array}{rrr}\text { Dry Branch } & \text { Congaree } & \text { Blck Creek } \\ -0.45335 & 0.170779 & 0.177335 \\ 0.170779 & -0.45335 & -0.4468 \\ 0.177335 & -0.4468 & -0.46927 \\ -0.21993 & -0.20291 & -0.19529 \\ 0.222477 & -0.40166 & -0.40994 \\ 0.311993 & -0.31214 & -0.33446\end{array}$

McBean Tobacco Rd Middendorf

0.2224770 .311993

$-0.40166-0.31214$

$-0.40994-0.33446$

$-0.15772-0.05947$

$-0.35841-0.27622$

$-0.27622-0.48698$ 
FIGURE $16 \mathrm{~d}$.

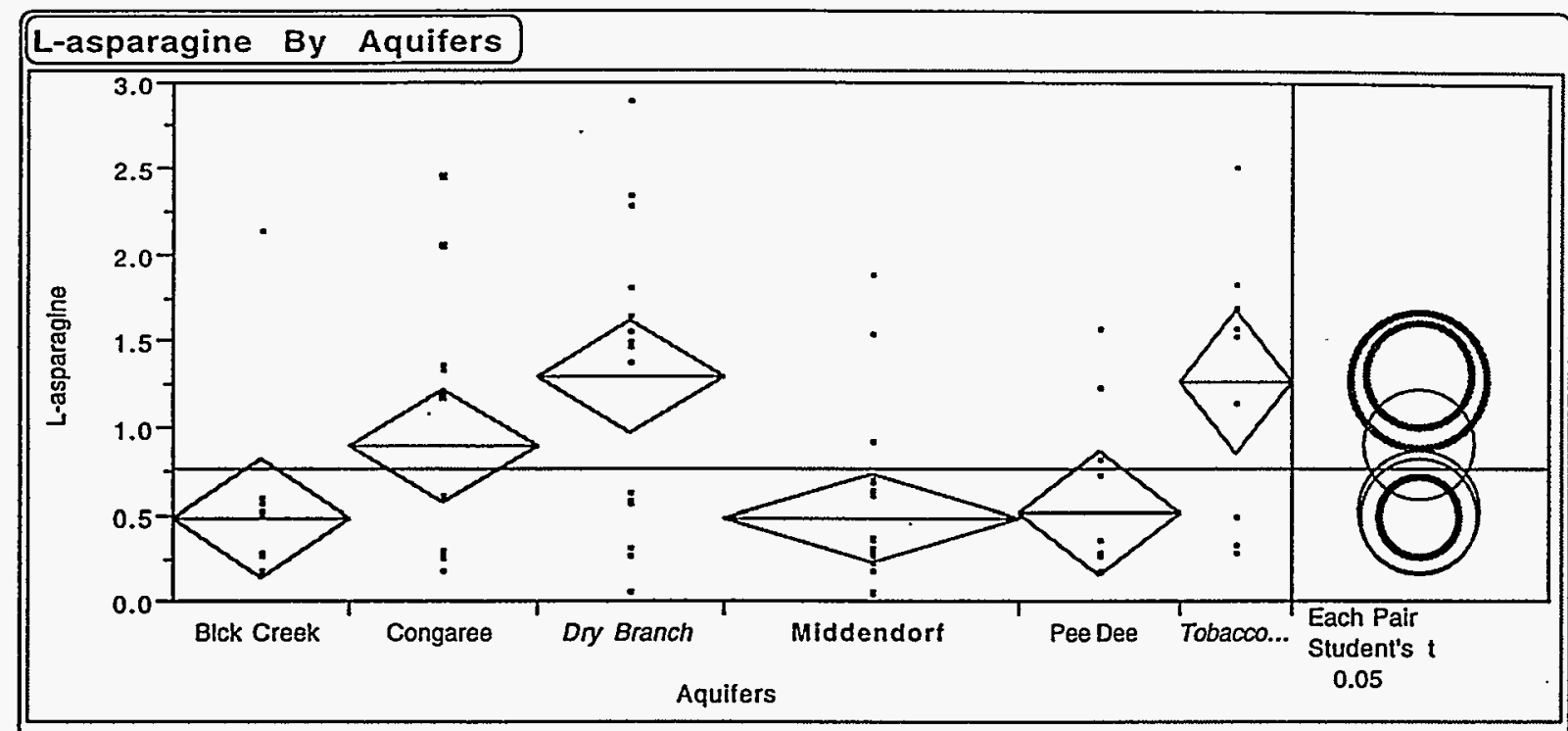

\section{Oneway Anova}

\begin{tabular}{|lr}
\hline Summary of Fit & \\
\hline RSquare & 0.233837 \\
RSquare Adj . & 0.188232 \\
Root Mean Square Error & 0.64731 \\
Mean of Response & 0.771589 \\
Observations (or Sum Wgts) & 90 \\
\hline
\end{tabular}

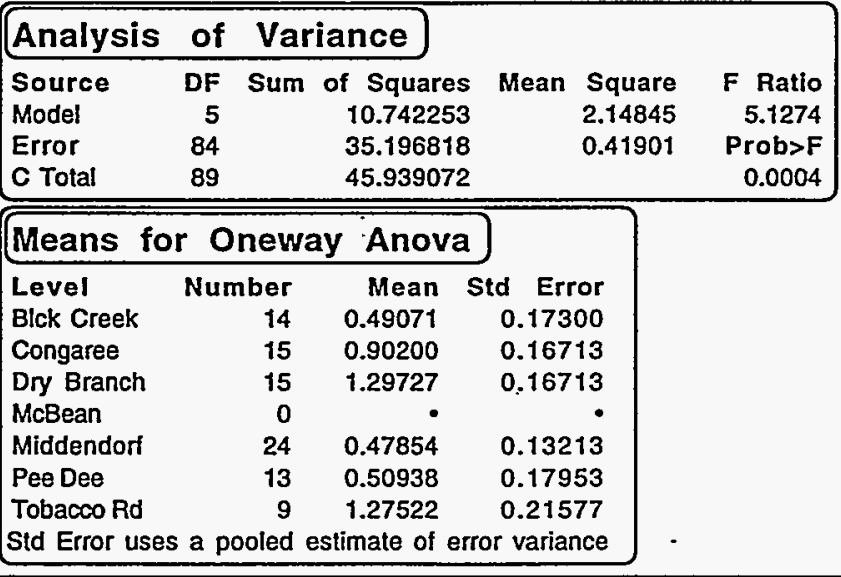

\section{Means Comparisons}

\section{Dry Branch}

Congaree

Blck Creek

McBean

Tobacco Rd

Pee Dee

Middendorf

Alpha $=0.05$

Comparisons for each pair using Student's $t$
Branch

0.000000

$-0.39527$

$-0.80655$

$-0.02204$

$-0.78788$

$-0.81872$
Congaree Blck Creek

0.395267

0.000000

$-0.41129$

0.373222

$-0.39262$

$-0.42346$
0.806552

0.411286

0.000000

0.784508

0.018670

$-0.01217$

\section{McBean Tobacco Rd}

\section{- 0.022044}

- $\quad-0.37322$

- 0.78451

-

0.000000

$-0.76584$

$-0.79668$
Pee Dee

0.787882

0.392615

$-0.01867$

0.765838

0.000000

$-0.03084$
Middendorf 0.818725 0.423458 0.012173

0.796681 0.030843 0.000000
1.98862

Abs(Dif)-LSD

Dry Branch

Congaree

BIck Creek

McBean

Tobacco Rd

Pee Dee

Middendorf
Dry Branch

$-0.47004$

$-0.07477$

0.328195

$-0.52071$

0.300101

0.395039
Congaree

$-0.07477$

$-0.47004$

$-0.06707$

$-0.16953$

$-0.09517$

$-0.00023$
Blck Creek 0.328195

$-0.06707$

$-0.48654$

0.234534

$-0.47713$

$-0.42073$
McBean Tobacco Rd $-0.52071$

$-0.16953$

0.234534

$-0.60682$

0.207648

0.293535
Pee Dee Middendorf

0.300101

$-0.09517$

$-0.47713$

0.207648

$-0.5049$

$-0.41245$
0.395039

$-0.00023$

$-0.42073$

0.293535

$-0.41245$

$-0.3716$

Positive values show pairs of means that are significantly different. 
FIGURE $16 \mathrm{e}$.

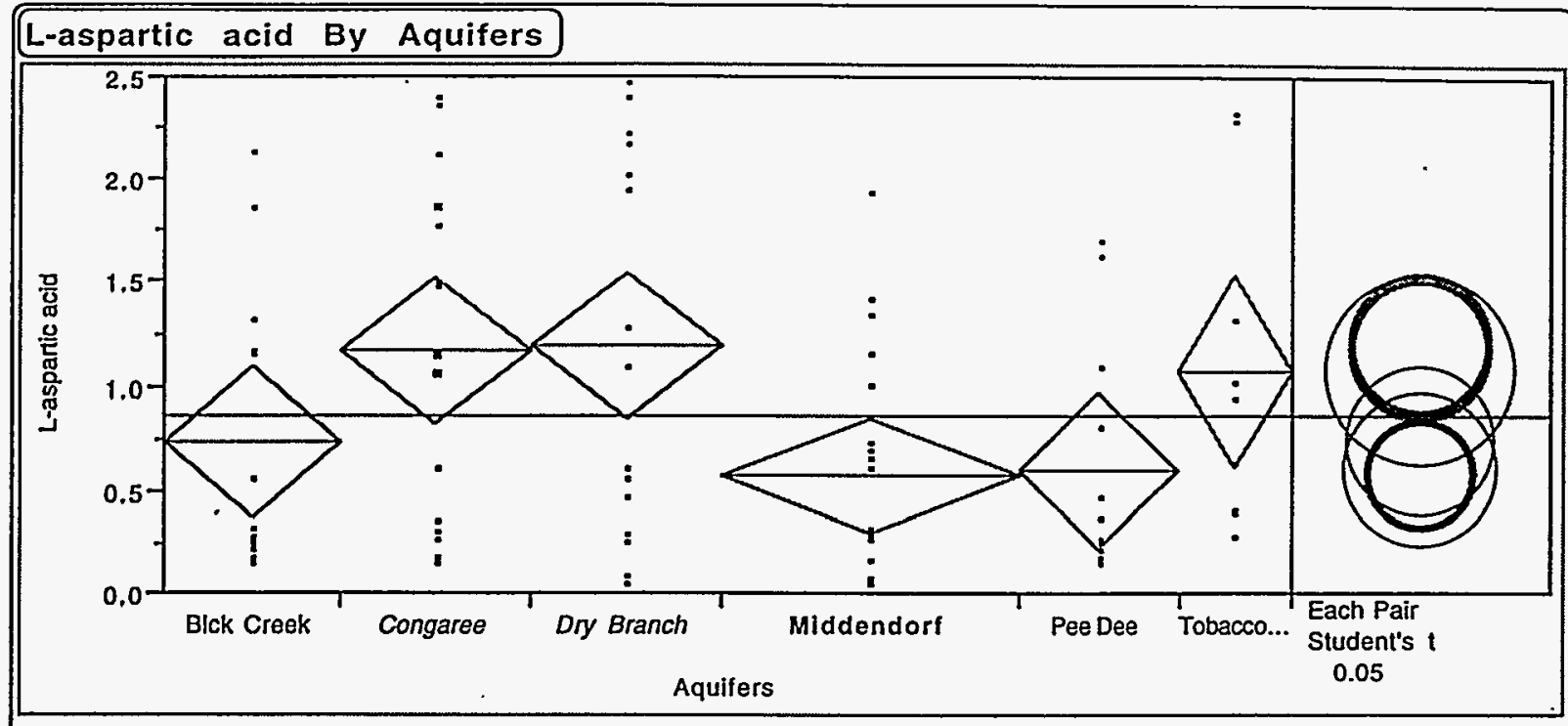

\section{Oneway Anova}

\begin{tabular}{|lr|}
\hline Summary of Fit & \\
RSquare & $\mathbf{0 . 1 4 1 6 0 7}$ \\
RSquare Adj & $\mathbf{0 . 0 9 0 5 1 3}$ \\
Root Mean Square Error & $\mathbf{0 . 7 0 1 1 9 5}$ \\
Mean of Response & $\mathbf{0 . 8 5 2 3 1 1}$ \\
Observations (or Sum Wgts) & 90 \\
\hline
\end{tabular}

\begin{tabular}{|lrrrrr|}
\hline Analysis & of & Variance & & & \\
Source & DF & Sum of Squares & Mean Square & F Ratio \\
Model & 5 & 6.813293 & 1.36266 & 2.7715 \\
Error & 84 & 41.300633 & 0.49167 & Prob $>$ F \\
C Total & 89 & 48.113925 & & 0.0229 \\
\hline
\end{tabular}

\begin{tabular}{|c|c|c|c|}
\hline \multicolumn{4}{|c|}{ Means for Oneway Anova } \\
\hline $\begin{array}{l}\text { Level } \\
\text { Blck Creek }\end{array}$ & $\begin{array}{r}\text { Number } \\
14\end{array}$ & $\begin{array}{r}\text { Mean } \\
0.72571\end{array}$ & $\begin{array}{l}\text { Std Error } \\
0.18740\end{array}$ \\
\hline Congaree & 15 & 1.17280 & 0.18105 \\
\hline Dry Branch & 15 & 1.19987 & 0.18105 \\
\hline McBean & 0 & - & - \\
\hline Mlddendorf & 24 & 0.57046 & 0.14313 \\
\hline Pee Dee & 13 & 0.58815 & 0.19448 \\
\hline Tobacco Rd & 9 & 1.06900 & 0.23373 \\
\hline Std Error use & pooled & & \\
\hline
\end{tabular}

\section{Means Comparisons}

Dif=Mean[j]-Mean[J] Dry Branch

Dry Branch

Congareo

Blck Creek

McBean

Tobacco Rd

Pee Dee

Middendorf

Alpha $=0.05$

Comparisons for each pair using Student's t 0.027067 $-0.02707$ $-0.47415$

\subsection{0}

$-0.44709$

$-0.13087 \quad-0.1038$

$-0.61171-0.58465$

$\begin{array}{ll}-0.62941 & -0.60234\end{array}$

\subsection{2}

0.447086

0.000000

0.343286

$-0.13756$

$-0.15526$
McBean Tobacco Rd

- $\quad 0.130867$

- -0.34329

- 0.000000

- $\quad-0.48085$
Pee Dee 0.611713

0.584646

0.137560

0.480846 0.000000

$-0.0177$
Middendorf 0.629408 0.602342 0.155256

0.498542 0.017696 0.000000
1.98862

Abs(DIf)-LSD

Dry Branch

Congaree

Blck Creek

McBean

Tobacco Rd

Pee Dee

Middendorf
Dry Branch $-0.50917$

$-0.4821$

$-0.04403$

$-0.45707$

0.083326

0.170452
Congaree

$-0.50917$

$-0.07109$

0.

0.056260

0.143386 $-0.04403$

$-0.07109$

$-0.52704$

0.

$-0.39952$

$-0.31368$
McBean Tobacco Rd $-0.45707$

$-0.48413$

$-0.25247$

$-0.65733$

$-0.12381$

$-0.04649$
Pee Dee 0.083326 0.056260 $-0.39952$

$-0.1238$

$-0.54693$

$-0.4625$
Middendorf

0.170452

0.143386

$-0.31368$

$-0.04649$

$-0.4625$

$-0.40253$

Positive values show pairs of means that are significantly different. 
FIGURE $16 f$.

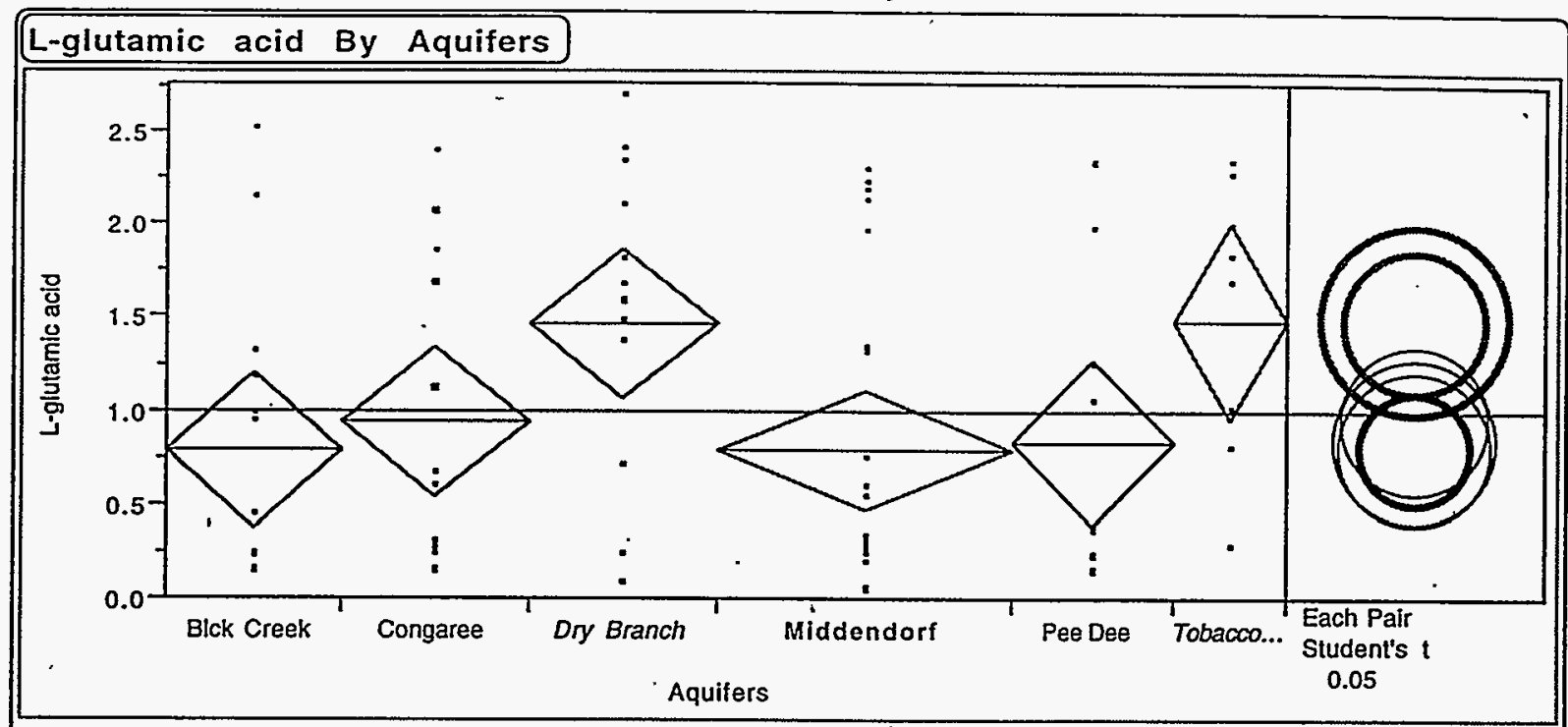

\section{Oneway Anova}

\section{Summary of Fit}

RSquare

RSquare Adj

0.120381

Root Mean Square Error

Mean of Response

0.068023

0.798969

1.003289

Observations (or Sum Wgts)

90

\begin{tabular}{|lrrrrr|}
\hline Analysis & of & Variance & & \\
Source & DF . Sum of Squares & Mean Square & F Ratio \\
Model & 5 & 7.338426 & 1.46769 & 2.2992 \\
Error & 84 & 53.621469 & 0.63835 & Prob>F \\
C Tolal & 89 & 60.959894 & & 0.0521 \\
\hline
\end{tabular}

Means for Oneway Anova

Level

Blck Creek

Congaree

Dry Branch

McBean

Middendorf

Pee Dee

Tobacco Rd

Mean Std Error

Number

$\begin{array}{rr}0.79207 & 0.21353\end{array}$

$0.93633 \quad 0.20629$

$15 \quad 1.46360$

0.20629

$\begin{array}{lrrr}\text { Tobacco Rd } & 9 & 1.47911 & 0.26632 \\ \text { Std Error uses a pooled estimate of error variance }\end{array}$

Means Comparisons

$$
\text { Di }
$$

Congaree

Blck Creek

McBean

Tobacco Rd

Pee Dee

Middendorf

0.79108

0.79108

0.16309

0.22159

Alpha $=0.05$

Comparisons for each pair using Student's $t$

$\begin{array}{rrr}\text { Branch } & \text { Congaree } & \text { Blck Creek } \\ 0.000000 & 0.527267 & 0.671529 \\ -0.52727 & 0.000000 & 0.144262 \\ -0.67153 & -0.14426 & 0.000000 \\ . & \bullet & \bullet \\ 0.015511 & 0.542778 & 0.687040 \\ -0.62437 & -0.0971 & 0.047159 \\ -0.67252 & -0.14525 & -0.00099\end{array}$

-

Pee Dee Middendorf 0.624369 0.097103 $-0.04716$ 0.672517 0.145250 0.000988 0.688028 0.048147 0.000000
1.98862 Abs(Dif)-LS Dry Branch Congaree Blck Creek McBean Tobacco Rd Pee Dee Middendorf

$\begin{array}{rrr}\text { Dry Branch } & \text { Congaree } & \text { Blck Creek } \\ -0.58016 & -0.0529 & 0.081096 \\ -0.0529 & -0.58016 & -0.44617 \\ 0.081096 & -0.44617 & -0.60053 \\ . & \bullet & \\ -0.6544 & -0.12714 & 0.008212 \\ 0.022305 & -0.50496 & -0.56481 \\ 0.149564 & -0.3777 & -0.53333\end{array}$

McBea

Positive values show pairs of means that are significantly different. 
FIGURE $16 \mathrm{~g}$.

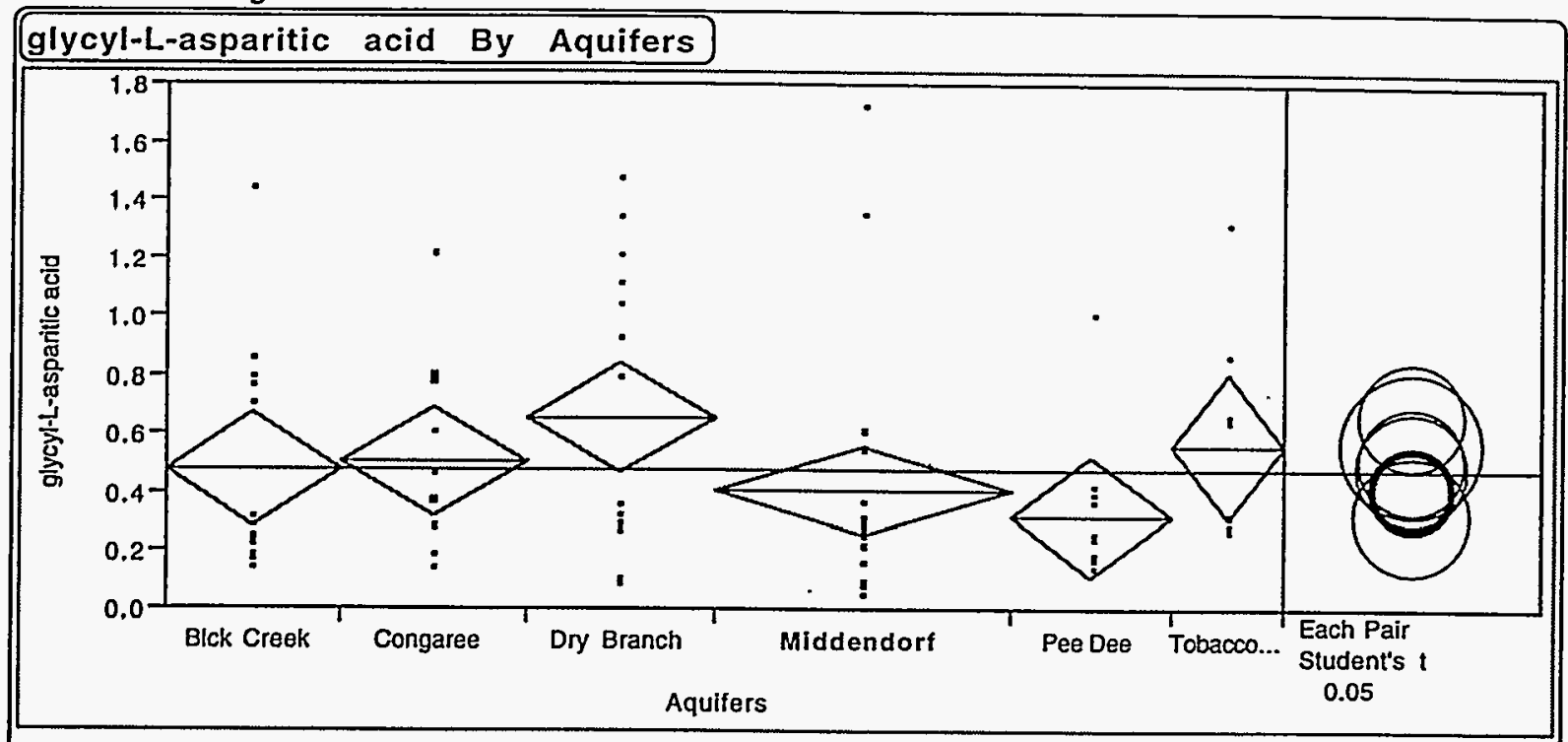

\section{Oneway Anova

Summary of Fit

RSquare

RSquare Adj

Root Mean Square Error

Mean of Response

Observations (or Sum Wgts)

0.018483

0.380665

0.476211

Analysis of Variance

\begin{tabular}{lrrrr} 
Source & DF & Sum of Squares & Mean Square & F Ratio \\
Model & 5 & 0.967392 & 0.193478 & 1.3352 \\
Error & 84 & 12.172099 & 0.144906 & Prob $>F$ \\
C Total & 89 & 13.139491 & & 0.2574 \\
\hline
\end{tabular}

Means' for Oneway Anova

Level Number Mean Std Error

$\begin{array}{llll}\text { Blck Creek } & 14 & 0.476857 & 0.10174\end{array}$

$\begin{array}{llll}\text { Congaree } & 15 & 0.500733 & 0.09829\end{array}$

$\begin{array}{lrrr}\text { Dry Branch } & 15 & 0.652267 & 0.09829\end{array}$

McBean

Middendort

Pee Dee

0 .

Tobacco Rd

$13 \quad 0.320462$

$9 \quad 0.555556$

0.07770

0.10558

\section{Means Comparisons}

\begin{tabular}{lrrr}
\hline Dif=Mean[i]-Mean[j] & Dry Branch & Congaree & Blck Creek \\
Dry Branch & 0.000000 & 0.151533 & 0.175410 \\
Congaroe & -0.15153 & 0.000000 & 0.023876 \\
Blck Creek & -0.17541 & -0.02388 & 0.000000 \\
McBean & - & - & - \\
Tobacco Rd & -0.09671 & 0.054822 & 0.078698 \\
Middendorf & -0.24718 & -0.09565 & -0.07177 \\
Pee Dee & -0.33181 & -0.18027 & -0.1564
\end{tabular}

$$
\begin{array}{rrrr}
\text { McBean } & \text { Tobacco Rd } & \text { Middendorf } & \text { Pee Dee } \\
- & 0.096711 & 0.247183 & 0.331805 \\
- & -0.05482 & 0.095650 & 0.180272 \\
- & -0.0787 & 0.071774 & 0.156396 \\
- & 0.000000 & 0.150472 & 0.235094 \\
- & -0.15047 & 0.000000 & 0.084622 \\
- & -0.23509 & -0.08462 & 0.000000
\end{array}
$$

Alpha $=0.05$

Comparisons for each pair using Student's t $t$ 1.98862 Abs(DIf)-LSD Dry Branch Congaree Blck Creek McBean Tobacco Rd Middendorf Pee Dee

$\begin{array}{rrr}\text { Dry Branch } & \text { Congaree } & \text { Blck. Creek } \\ -0.27642 & -0.12488 & -0.1059 \\ -0.12488 & -0.27642 & -0.25743 \\ -0.1059 & -0.25743 & -0.28612 \\ -0.22247 & -0.26436 & -0.24473 \\ -0.00198 & -0.15351 & -0.1828 \\ 0.044954 & -0.10658 & -0.13517\end{array}$
McBean Positive values show pairs of means that are significantly different.

\begin{tabular}{rrrr} 
- & Tobacco Rd & Middendorf & Pee Dee \\
\hline & -0.22247 & -0.00198 & 0.044954 \\
$:-0.26436$ & -0.15351 & -0.10658 \\
- & -0.24473 & -0.1828 & -0.13517 \\
\hline & -0.35685 & -0.14541 & -0.09316 \\
$:$ & -0.14541 & -0.21853 & -0.17606 \\
- & -0.09316 & -0.17606 & -0.29692
\end{tabular}




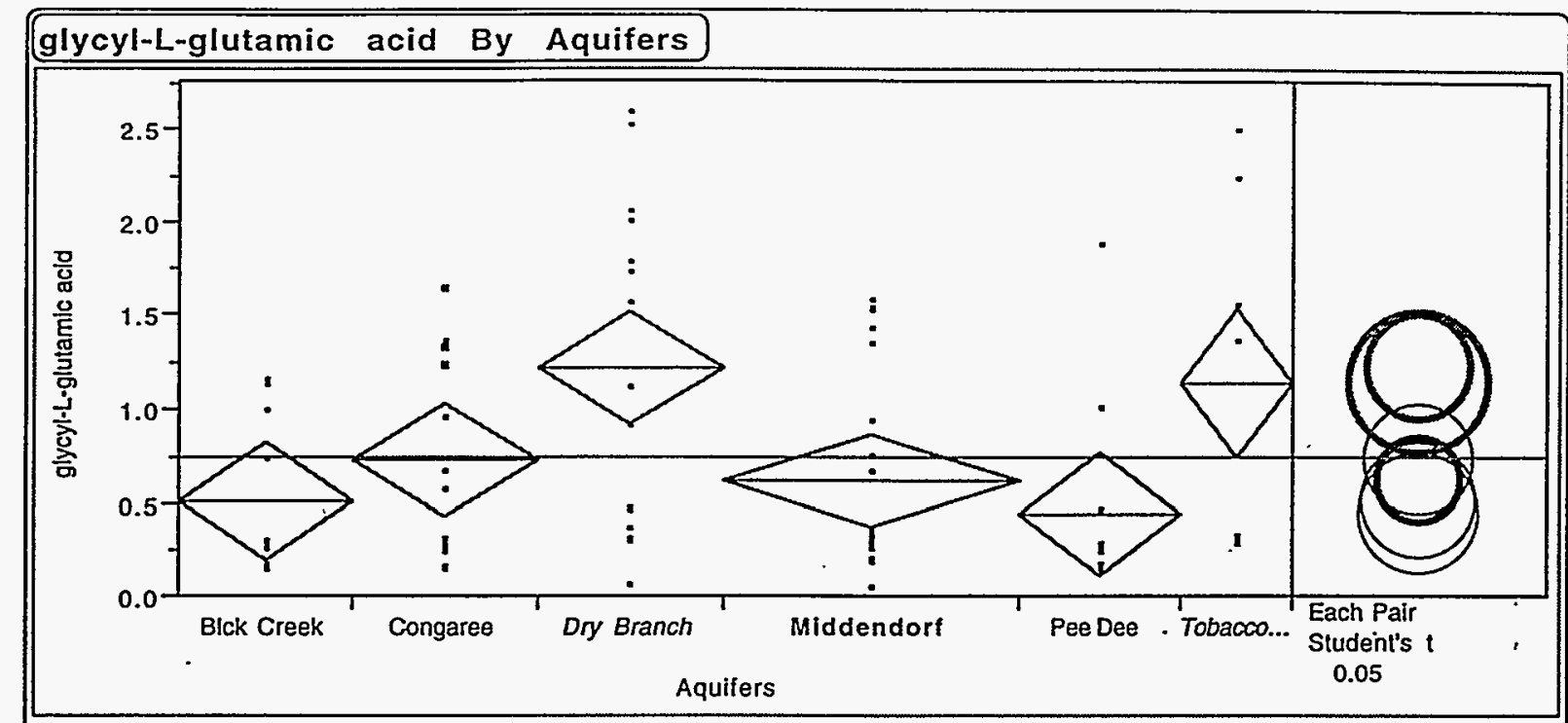

\section{Oneway Anova}

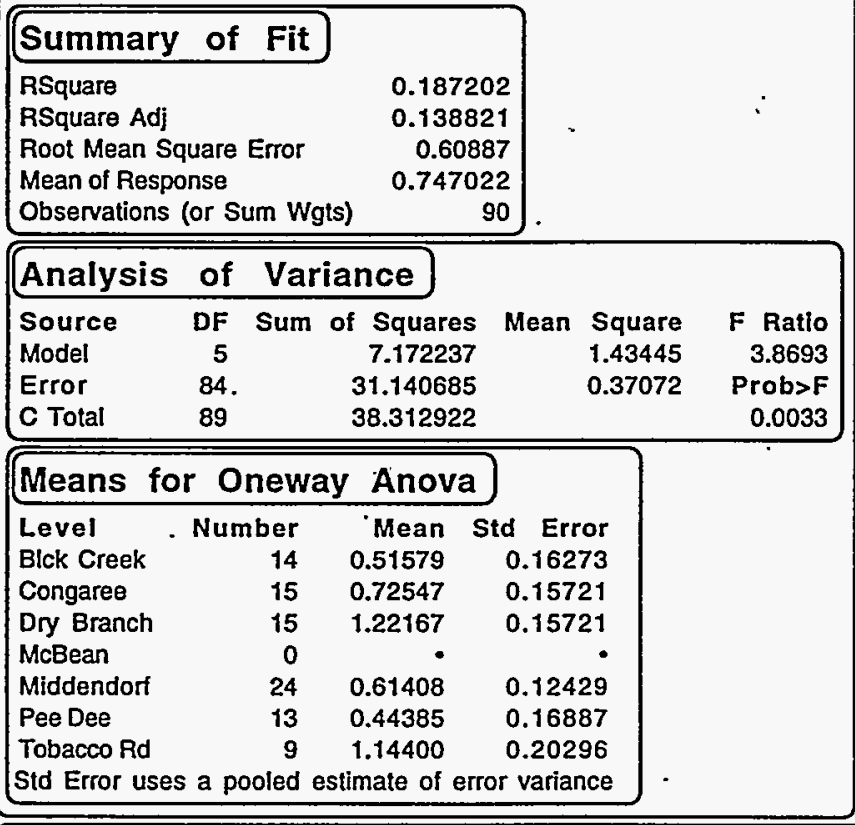

\section{Means Comparisons}

Dif=Mean[i]-Mean[i] Dry Branch Congaree Bick Creek

Dry Branch

Congaree

Branch

$0.000000 \quad 0.496200$

0.705881

$-0.4962 \quad 0.000000$

0.209681

Blck Creek

McBean

$-0.70588-0.20968$

0.000000

Tobacco Rd

Middendorf

$-0.07767 \quad 0.418533$

$-0.60758-0.11138$

0.628214

0.628214
0.098298

$\begin{array}{lll}-0.77782 & -0.28162 & -0.07194\end{array}$

$\begin{array}{rrrr}\text { McBean } & \text { Tobacco Rd } & \text { Middendorf } & \text { Pee Dee } \\ - & 0.077667 & 0.607583 & 0.777821 \\ \text { - } & -0.41853 & 0.111383 & 0.281621 \\ \text { - } & -0.62821 & -0.0983 & 0.071940 \\ \text { - } & 0.000000 & 0.529917 & 0.700154 \\ \text { - } & -0.52992 & 0.000000 & 0.170237 \\ & -0.70015 & -0.17024 & 0.000000\end{array}$

Alpha $=0.05$

Comparisons for each pair using Student's $t$

1.98862

Abs(DIf)-LSD

Dry Branch

Dry Branch

$-0.44213$

0.054075

0.255930

Congaree

0.054075

Blck Creek

Bick Creek

$\begin{array}{ll}-0.44213 & -0.24027\end{array}$

McBean

$-0.24027$

$-0.45764$

Tobacco Rd

Middendorf

$\begin{array}{lll}-0.43286 & -0.09199 & 0.110900\end{array}$

$0.209057-0.28714$

$0.319006-0.17719$

$-0.30889$

$-0.39442$

McBean Tobacco Rd

$-0.43286$

$-0.09199$

0.110900

$-0.57078$

0.056650

0.175112
Middendorf

0.209057

$-0.28714$

$-0.30889$

0.056650

$-0.34953$

$-0.24673$
Pee Dee

0.319006

$-0.17719$

$-0.39442$

0.175112

$-0.24673$

$-0.47492$

Positive values show pairs of means that are signilicantly different. 
FIGURÉ $16 i$.

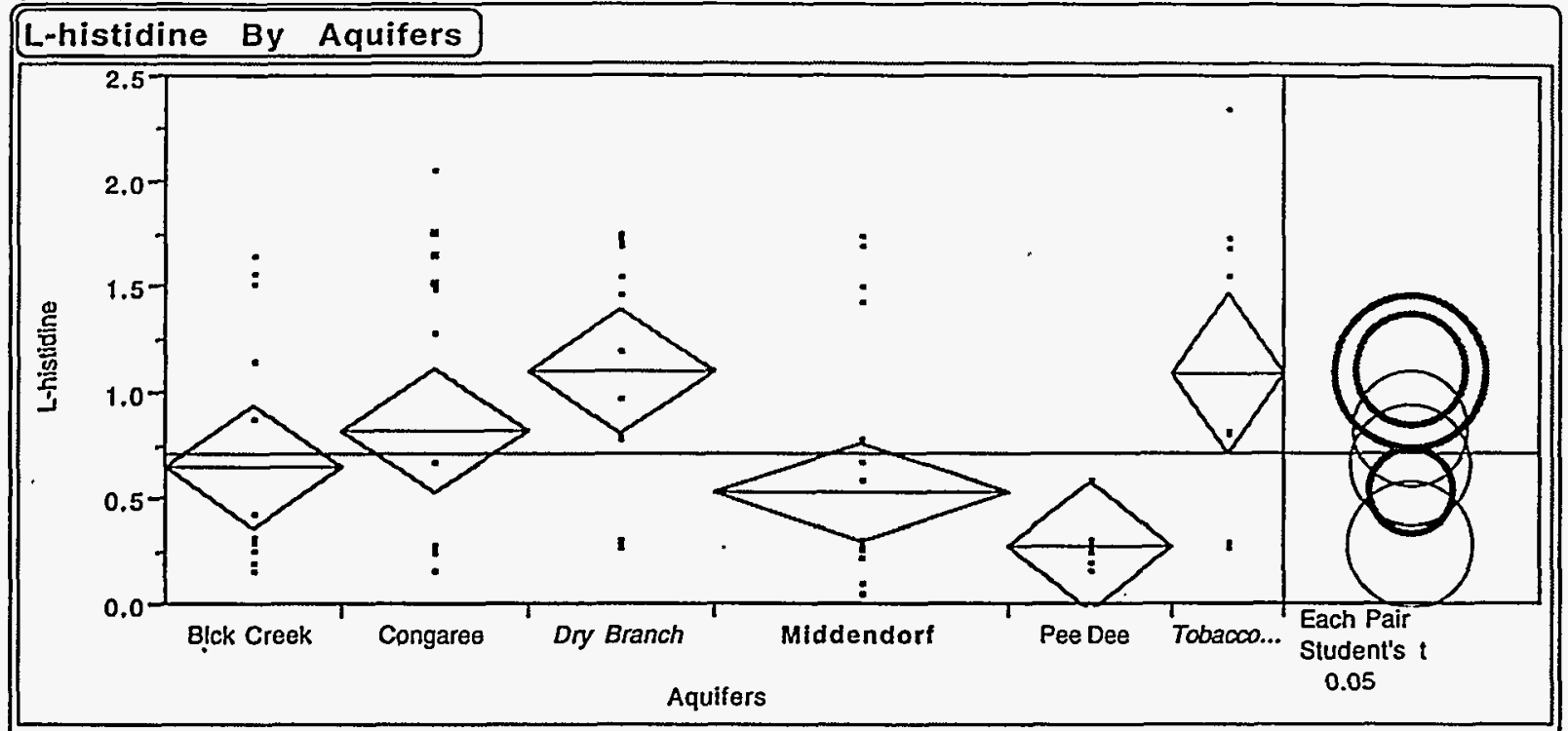

\section{Oneway Anova}

Summary of Fit

RSquare

RSquare AdJ

Root Mean Square Error

Mean of Response

Observatlons (or Sum Wgts)

0.207881

0.160731

0.568141

0.711489

Analysis of Variance

Source DF Sum of Squares Mean Square F Ratio

$\begin{array}{rrrrr}\text { Model } & 5 & 7.115669 & 1.42313 & 4.4089 \\ \text { Error } & 84 & 27.113834 & 0.32278 & \text { Prob }>F\end{array}$

\begin{tabular}{llll} 
C Total & 89 & 34.229502 & 0.0013 \\
\hline
\end{tabular}

Means for Oneway Anova

Level Number Mean std Error

$\begin{array}{lrrr}\text { Blck Creek } & 14^{\circ} & 0.65236 & 0.15184\end{array}$

$\begin{array}{llll}\text { Congaree } & 15 & 0.82267 & 0.14669\end{array}$

$\begin{array}{llll}\text { Dry Branch } & 15 & 1.10300 & 0.14669\end{array}$

McBean

Middendorf

Pee Dee

0 .

$\begin{array}{lll}24 & 0.52917 & 0.11597\end{array}$

Tobacco Rd

90.27169

0.15757

Std Error uses' a pooled estimate of error variance

\section{Means Comparisons}

\begin{tabular}{|c|c|c|c|c|c|c|c|}
\hline $\begin{array}{l}\text { DIf = Moan[l]-Mean[]] } \\
\text { Dry Branch }\end{array}$ & $\begin{array}{r}\text { Dry Branch } \\
0.000000\end{array}$ & $\begin{array}{r}\text { Congaree } \\
0.280333\end{array}$ & $\begin{array}{r}\text { Blck Creek } \\
0.450643\end{array}$ & McBean & $\begin{array}{r}\text { Tobacco Rd } \\
0.015889\end{array}$ & $\begin{array}{r}\text { Middendorf } \\
0.573833\end{array}$ & $\begin{array}{l}\text { Pee Dee } \\
0.831308\end{array}$ \\
\hline Congaree & -0.28033 & 0.000000 & 0.170310 & $\bullet$ & -0.26444 & 0.293500 & 0.550974 \\
\hline Blck Creek & -0.45064 & -0.17031 & 0.000000 & - & -0.43475 & 0.123190 & 0.380665 \\
\hline McBean & - & - & - & - & - & - & - \\
\hline Tobacco Rd & -0.01589 & 0.264444 & 0.434754 & - & 0.000000 & 0.557944 & 0.815419 \\
\hline MIddendorf & -0.57383 & -0.2935 & -0.12319 & - & -0.55794 & 0.000000 & 0.257474 \\
\hline Pee Dee & -0.83131 & -0.55097 & -0.38066 & - & -0.81542 & -0.25747 & 0.000000 \\
\hline
\end{tabular}

Alpha $=0.05$

Comparisons for each pair using Student's $t$ $t$

1.98862

Abs(DIf)-LSD
Dry Branch

Dry Branch

Branch Congaree

Blck Creek

McBean Tobacco Rd

Middendorf

Congaree

Blck Creek

McBean

Tobacco Rd

Mlddendorf

Pee Dee

$-0.41255$

$-0.13222$

0.030791

$-0.13222-0.41255$

$-0.24954$

$-0.24954$

$-0.42703$

$-0.46048$

$-0.21193$

$-0.04796$

0.201966

$-0.07837$

$-0.25676$

0.403184

0.122851

$-0.0545$

$-0.46048$

- $\quad-0.21193$

- $\quad 0.04796$

Positive values show pairs of means that are significantly dilferent.

-0.5326
0.116336

0.325498
$-0.07837 \quad 0.122851$

$-0.25676 \quad-0.0545$

$0.116336 \quad 0.325498$

$-0.32615-0.1316$

$-0.1316 \quad-0.44315$ 
FIGURE $16 j$.

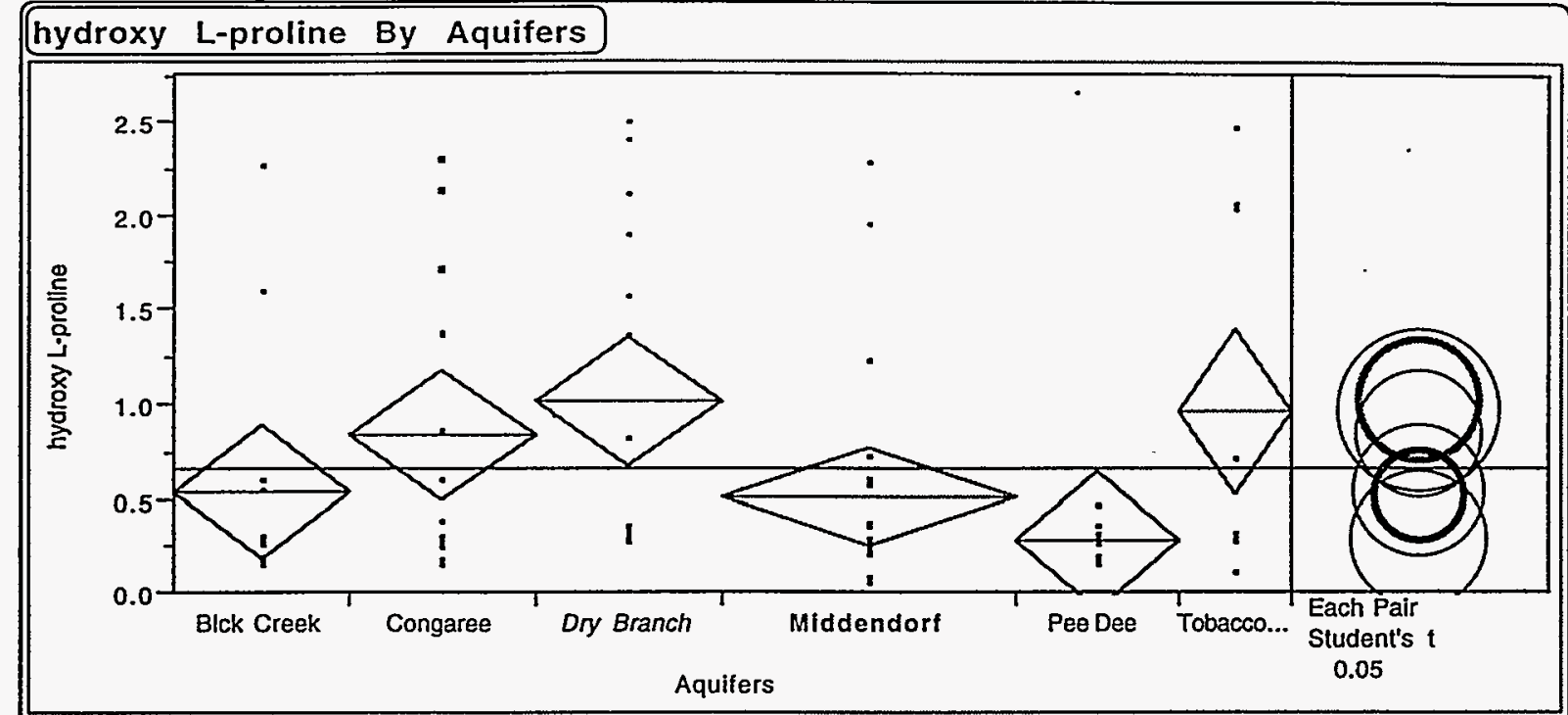

\section{Oneway Anova \\ Summary of Fit}

RSquare

-RSquare Adj

Root Mean Square Error

Mean of Response

Observations (or Sum Wgts)

\begin{tabular}{|lrrrrr|}
\hline Analysis & of & \multicolumn{2}{c|}{ Variance } & & \\
\hline Source & DF & Sum of Squares & Mean Square & F Ratio \\
Model & .5 & 5.882178 & 1.17644 & 2.6326 \\
Error & 84 & 37.537675 & 0.44688 & Prob $>$ F \\
C Total & 89 & 43.419853 & & 0.0292 \\
\hline
\end{tabular}

Means for Oneway Anova

Level Number Mean Std Error

$\begin{array}{llll}\text { Blck Creek } & 14 & 0.53679 & 0.17866\end{array}$

$\begin{array}{llll}\text { Congaree } & 15 & 0.83073 & 0.17260\end{array}$

$\begin{array}{llll}\text { Dry.Branch } & 15 & 1.01813 & 0.17260\end{array}$

McBean

Middendorf

Pee Dee

$\begin{array}{lrrr}\text { Tobacco Rd } & 9 & 0.95778 & 0.22283\end{array}$

Std Error uses a pooled estimate of error variance

\section{Means Comparisons}

Dif=Mean[i]-Mean[i]] Dry Branch

Dry Branch

Congaree

Blck Creek

McBean

Tobacco Rd

Middendort

Pee Dee

Alpha $=0.05$

Comparisons for each pair using Student's t

$$
\text { t }
$$

$$
1.98862
$$

Abs(Dif)-LSD

Dry Branch

Congaree

Blck Creek

McBean

Tobacco Rd

Middendorf

Pee Dee

Dry Branch

$-0.48542$

Congaree Blck Creek

$-0.29802$

$-0.01266$

McBea

$-0.29802$

$-0.01266$

$-0.48542$

$-0.20006$

$-0.50016$

$-0.20006$

$-0.50245$

0.073960

$-0.43347$

$-0.11344$

0.241316

0.053916

$-0.14698$

$-0.4169$

$-0.24832$ lck Creek

0.481348

0.293948

0.000000

0.420992

$-0.03016$

0.26371
McBean Tobacco Rd Middendorf

Pee Dee

- 0.060356

$-0.12704$

$-0.42099$

0.000000

$-0.45115$

$-0.6847$
0.511508

0.324108

0.030161

0.451153

0.000000

$-0.23355$
0.745056

0.557656

0.263709

0.684701

0.233548

0.000000

Positive values show pairs of means that are signilicantly different. 
FIGURE $16 \mathrm{~K}$.

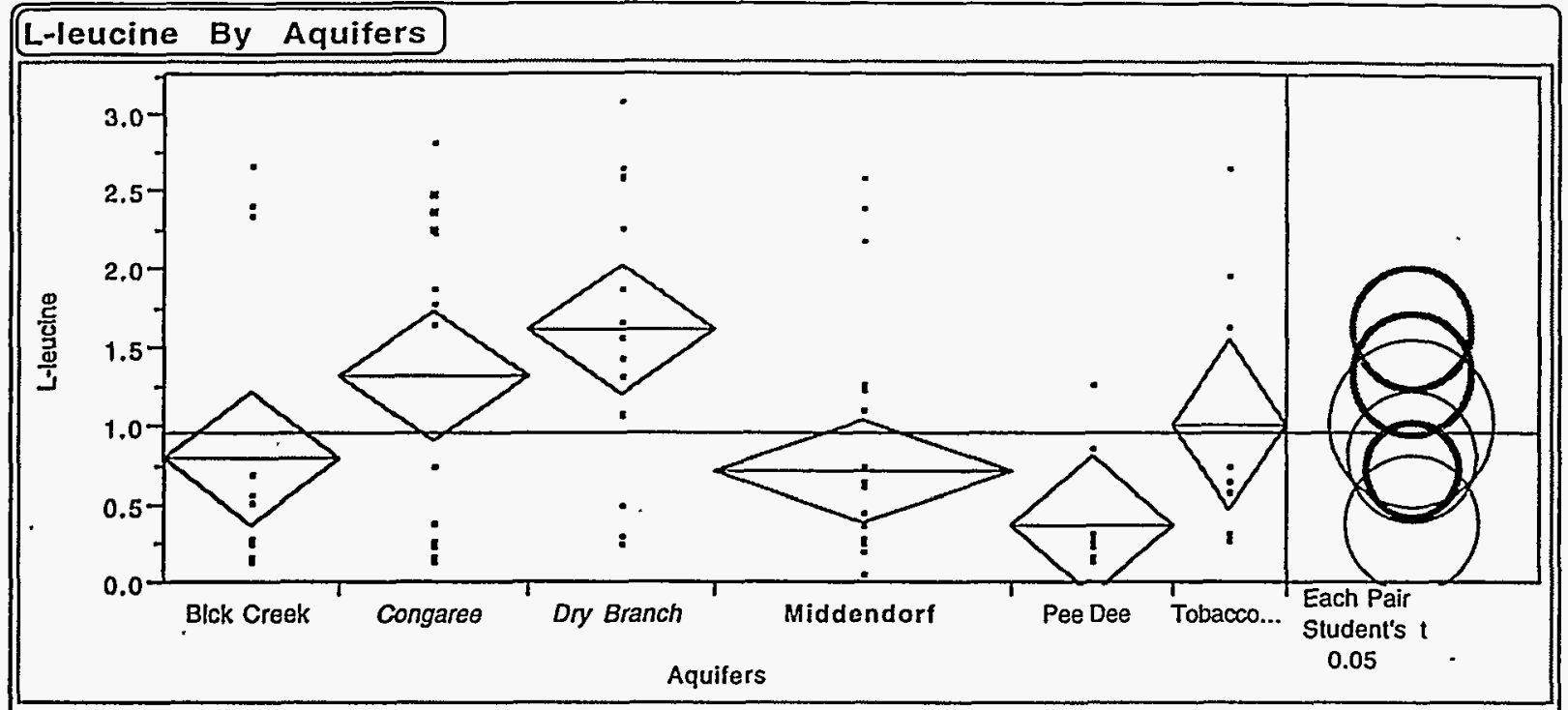

\section{Oneway Anova}

\section{Summary of Fit}

RSquare

0.210132

RSquare Ad]

Root Mean Square Error

Mean of Response

0.814308

0.9557

Observatlons (or Sum Wgts)

Analysis of Variance

Sourco DF Sum of Squares Mean Square F Ratio

Model $\quad 5^{\circ} \quad 14.818189 \quad 2.96364 \quad 4.4694$

\begin{tabular}{llrrr} 
Error & 84 & 55.700160 & 0.66310 & Prob $>F$ \\
C Total & 89 & 70.518349 & & 0.0012 \\
\hline
\end{tabular}

Means for Oneway Anova

Lovel Number Mean Std Error

$\begin{array}{llll}\text { Blck Creek } & 14 & 0.78400 & 0.21763\end{array}$

$\begin{array}{lll}\text { Congaree } & 15,1.31553 & 0.21025\end{array}$

$\begin{array}{llll}\text { Dry Branch } & 15 & 1.61627 & 0.21025\end{array}$

McBean

Middendorf

Pee Dee

Tobacco Rd

$\begin{array}{lll}9 & 1.00844 & 0.27144\end{array}$

Std Error uses a pooled estimate of error variance

\section{Means Comparisons}

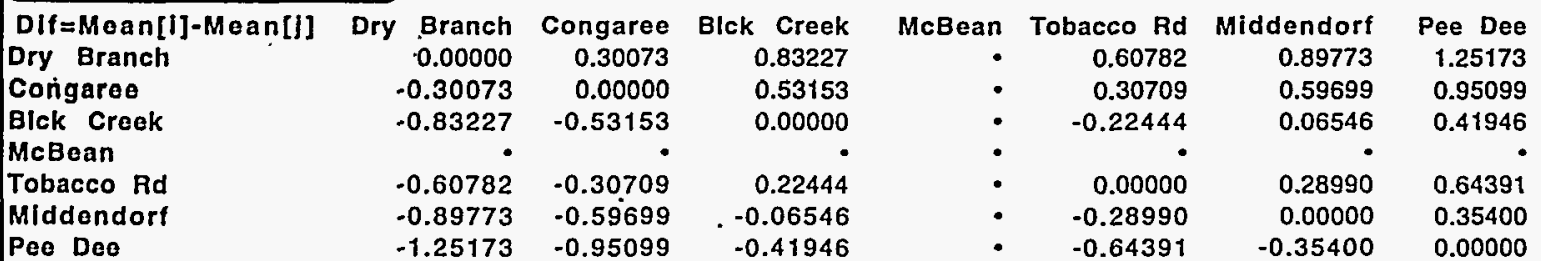

Alpha $=0.05$

$\begin{array}{lll}-1.25173 & -0.95099 & -0.41946\end{array}$

Comparisons for each pair using Student's t

1.98862

Abs(DIf)-LSD

Dry Branch

Congaree

Blck Croek

McBean

Tobacco Rd

Mlddendorf

Dry Branch

Congaree

$-0.5913$

$-0.29057$

$-0.29057$

Blck Creek

$-0.5913-0.07023$

$-0.07023$

$-0.61206$

.

$\begin{array}{ll}-0.07495 & -0.37569\end{array}$

0.364733

Peo Dee

$0.638105 \quad 0.337372$

$-0.46742$

$-0.47912$

$-0.20425$

McBean Tobacco Rd

$-0.07495$

$-0.37569$

$-0.46742$

- $\quad$.

-0.76337
-0.34305

$-0.05829$
Middendorf Pee Dee 0.3647330 .638105 $0.063999 \quad 0.337372$

$-0.47912-0.20425$

$\begin{array}{ll}-0.34305 & -0.05829\end{array}$

$-0.46747-0.20365$

$\begin{array}{ll}-0.20365 & -0.63516\end{array}$

Positive values show pairs of means that are significantly different. 
FIGURE 161.

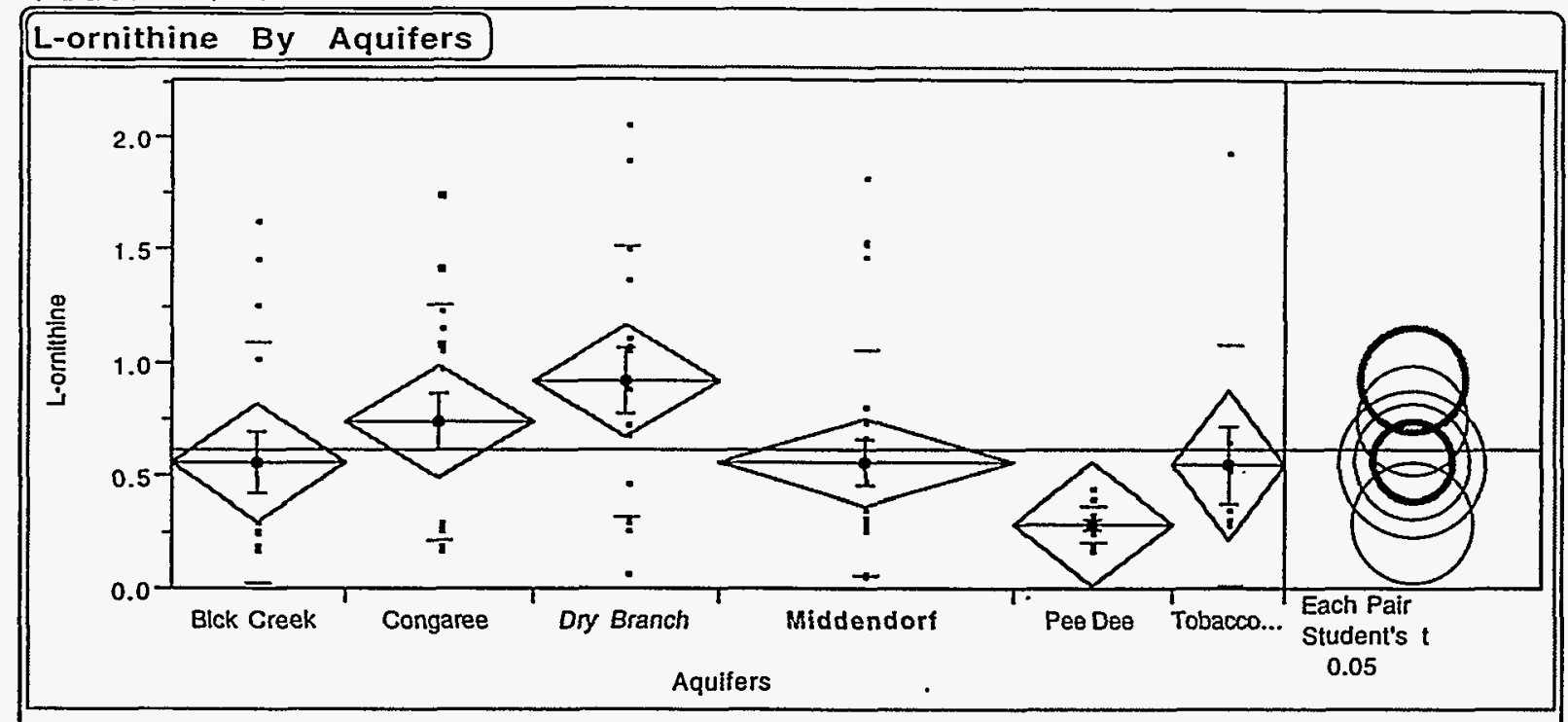

\section{Oneway Anova}

Summary of Fit

RSquare

RSquare Adj

Root Mean Square Error

Mean of Response

0.081788

0.496646

0.608644

Observations (or Sum Wgts)

\begin{tabular}{|lrrrrr|}
\hline Analysis & of & Variance & & \\
Source & DF & Sum of Squares & Mean Square & F Ratio \\
Model & 5 & 3.188660 & 0.637732 & 2.5855 \\
Error & 84 & 20.719247 & 0.246658 & Prob $>$ F \\
C Total & 89 & 23.907907 & & 0.0317 \\
\hline
\end{tabular}

Means for Oneway Anova

Level Number Mean Std Error

$\begin{array}{llll}\text { Blck Creek } & 14 & 0.559786 & 0.13273\end{array}$

$\begin{array}{llll}\text { Congaree } & 15 & 0.741800 & 0.12823\end{array}$

$\begin{array}{llll}\text { Dry Branch } & 15 & 0.918600 & 0.12823\end{array}$

McBean

Middendorf

0

Pee Dee

$\begin{array}{lll}24 & 0.557833 & 0.10138\end{array}$

$13 \quad 0.286077 \quad 0.13774$

Tobacco Rd

90.547556

0.16555

Std Error uses a pooled estimate of error variance

\section{Means Comparisons}

Dif=Mean[i]-Mean[j] Dry Branch

Dry Branch

Congaree

Blck Creek

McBean

Middendorf

Tobacco Rd

Pee Dee

Alpha $=0.05$

Comparisons for each pair using Student's $t$
$0.176800 \quad 0.358814$

$\begin{array}{lll}-0.1768 & 0.000000 & 0.182014\end{array}$

$-0.35881$

$-0.36077$

$-0.37104$

$-0.63252$
$-0.18201$

0.000000

$-0.18397$

$-0.19424$

$-0.45572$

$-0.00195$
$-0.01223$

$-0.27371$
McBean Middendorf Tobacco Rd 0.371044

0.194244

0.012230

0.010278

0.000000

$-0.26148$
Pee Dee 0.632523 0.455723 0.273709

0.271756

0.261479 0.000000
1.98862

Abs(Dif)-LSD

Dry Branch

Congaree

Blck Creek

McBean

Middendorf

Tobacco Rd

Pee Dee
Dry Branch

$\begin{array}{rrr}\text { Branch } & \text { Congaree } & \text { Blck Creek } \\ -0.36064 & -0.18384 & -0.0082 \\ -0.18384 & -0.36064 & -0.185 \\ -0.0082 & -0.185 & -0.37329 \\ - & - & - \\ 0.035695 & -0.14111 & -0.33019 \\ -0.04538 & -0.22218 & -0.40974 \\ 0.258274 & 0.081474 & -0.1067\end{array}$

McBean Middendorf Tobacco Rd

$-0.04538$

$-0.22218$

$-0.40974$

$-0.37576$

$-0.46558$

$-0.16679$
Pee Dee

0.258274

0.081474

$-0.1067$

$-0.06836$

$-0.16679$

$-0.38738$

Positive values show pairs of means that are significantly different. 
FIGURE $16 \mathrm{~m}$.

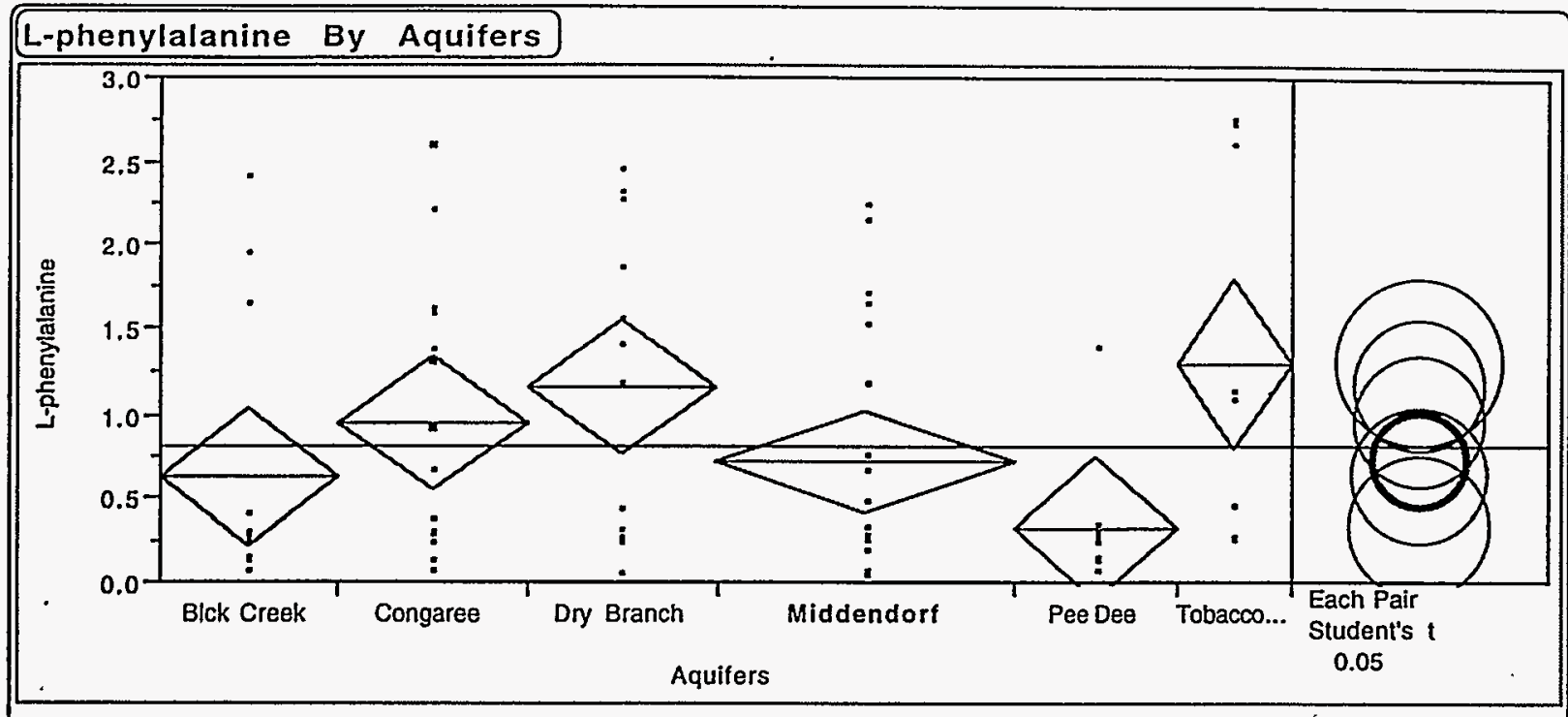

\section{Oneway Anova}

\section{Summary of Fit}

RSquare

RSquare Adj

Root Mean Square Error

Mean of Response

Observations (or Sum Wgts)

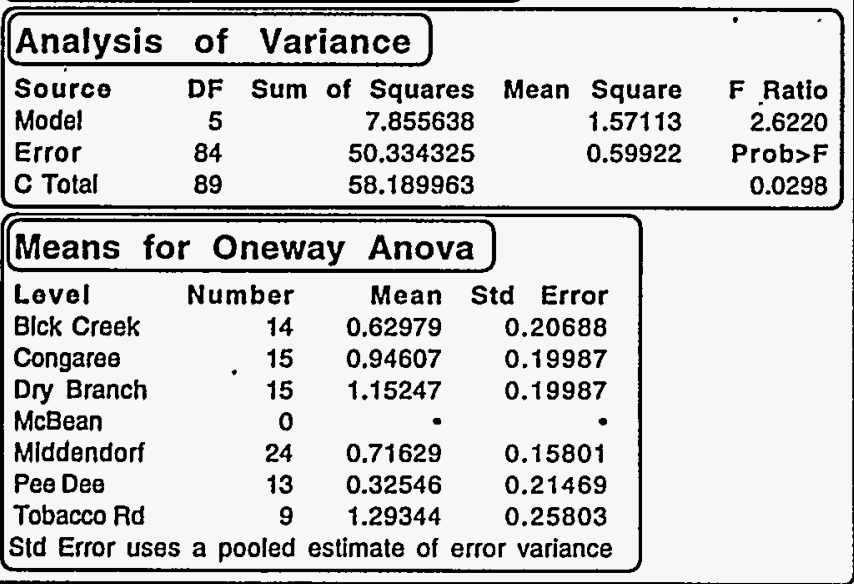

\section{Means Comparisons}

\begin{tabular}{|c|c|c|c|c|c|c|c|}
\hline Dif=Mean[i]-Mean[j] & Dry Branch & Congaree & Blck Creek & McBean & Tobacco Rd & Middendorf & Pee Dee \\
\hline Congaree & $\begin{array}{r}0.00064 \\
-0.2064\end{array}$ & 0.000000 & $\begin{array}{l}0.522081 \\
0.316281\end{array}$ & $\bullet$ & $\begin{array}{l}-0.14098 \\
-0.34738\end{array}$ & $\begin{array}{l}0.4367 / 5 \\
0.229775\end{array}$ & $\begin{array}{l}0.827005 \\
0.620605\end{array}$ \\
\hline Blck Creak & -0.52268 & -0.31628 & 0.000000 & 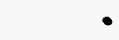 & -0.66366 & -0.08651 & 0.304324 \\
\hline McBean & . & • & • & - & - & - & \\
\hline obacco Rd & 0.140978 & 0.347378 & 0.663659 & $\bullet$ & 0.000000 & 0.577153 & 0.967983 \\
\hline Middendorf & -0.43617 & -0.22977 & 0.086506 & - & -0.57715 & 0.000000 & 0.390830 \\
\hline Pee Dee & -0.82701 & -0.62061 & -0.30432 & $\bullet$ & -0.96798 & -0.39083 & 0.000000 \\
\hline
\end{tabular}

Alpha $=0.05$

Comparisons for each pair using Student's t

$$
t
$$

1.98862

Abs(DIf)-LSD

Dry Branch

Congareo

Blck Creek Dry Branch
-0.5621

$-0.3557$

$-0.04937$

$-0.50808$

$-0.07049$

0.243687

$\begin{array}{rr}\text { Congaree } & \text { Blck Creek } \\ -0.3557 & -0.04937 \\ -0.5621 & -0.25577 \\ -0.25577 & -0.58183 \\ -0.30168 & 0.005967 \\ -0.27689 & -0.43118 \\ 0.037287 & -0.28859\end{array}$

McBean Tobacco Rd Middendorf

$-0.50808$

$-0.30168$

0.005967

$-0.72567$

$-0.02454$

0.300466 $\begin{array}{ll}-0.07049 & 0.243687\end{array}$

$-0.27689 \quad 0.037287$

$-0.43118 \quad-0.28859$

$-0.024540 .300466$

$-0.44438 \quad-0.13928$

$-0.13928-0.60379$

Positive values show pairs of means that are significantly different. 
FIGURE 160.

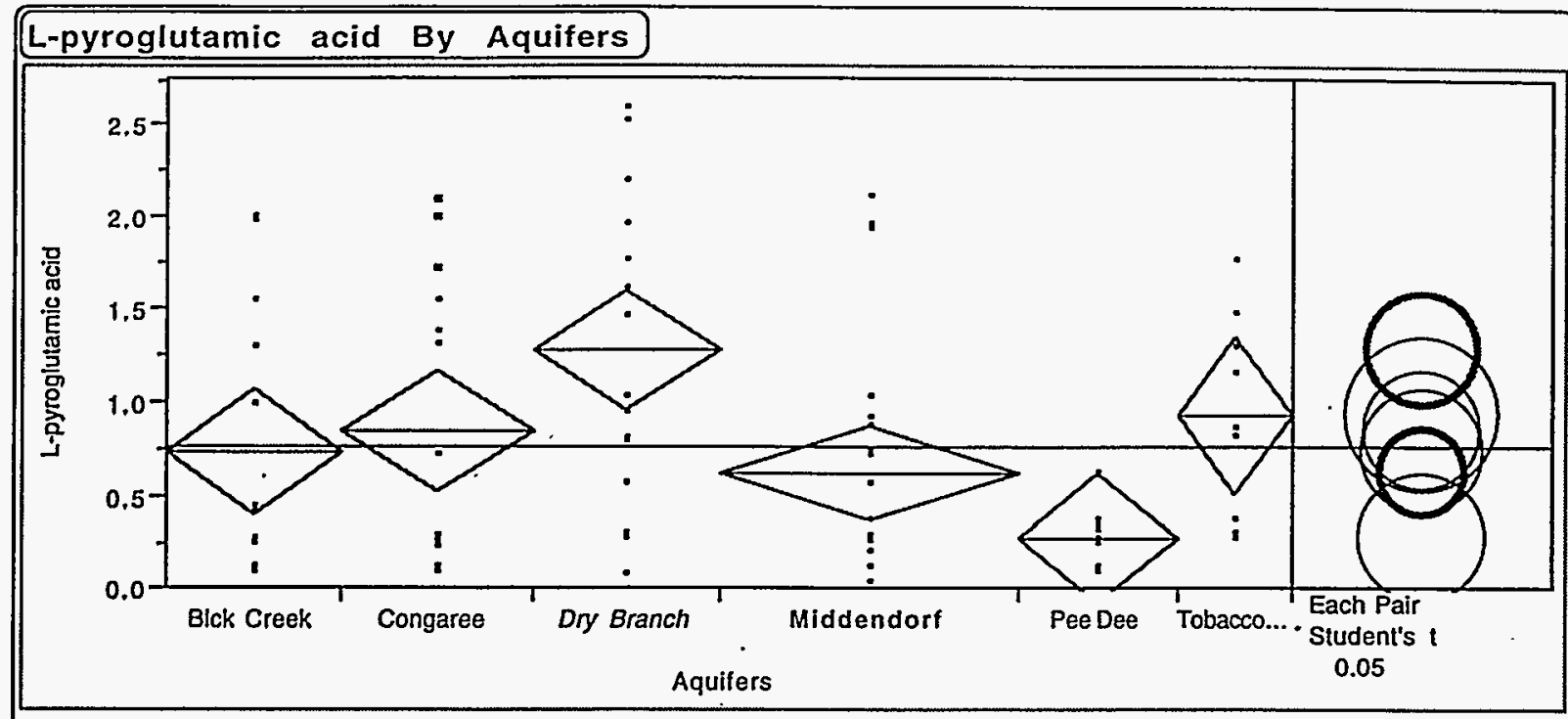

\section{Oneway Anova}

\section{Summary of Fit}

RSquare

RSquare Adl

Root Mean Square Error

Mean of Response

Observations (or Sum Wgts)

\begin{tabular}{|lrrrrr|}
\hline Analysis & of & Variance & & \\
Sourco & DF & Sum of Squares & Mean Square & F Ratio \\
Model & 5 & 7.802711 & 1.56054 & 3.7916 \\
Error & 84 & 34.572881 & 0.41158 & Prob $>$ F \\
C Tolal & 89 & 42.375592 & & 0.0038 \\
\hline
\end{tabular}

Means for Oneway Anova

Lovel Number Mean Std Error

$\begin{array}{llll}\text { Blck Creek } & 14 & 0.72836 & 0.17146\end{array}$

$\begin{array}{llll}\text { Congaree } & 15 & 0.85160 & 0.16565\end{array}$

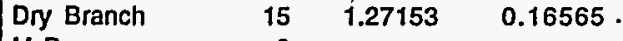

McBean

Middendorf

Pee Dee

Tobacco Rd

0.62412

0.27785

0.13096

$\begin{array}{llll}13 & 9 & 0.93556 & 0.21385\end{array}$

Std Error uses a pooled estimate of error variance

\section{Means Comparisons}

Dif=Mean[I]-Mean[I] Dry Branch Dry Branch

Congareo

Blck Creek

McBean

Tobacco Rd

Middendorf

Pee Dee

Alpha $=0.05$

Comparisons for each pair using Student's t 1

198862

Abs(Dlf)-LSD

Dry Branch

Congareo

Blck Creek

McBean

Tobacco Rd

Mlddendorf

Peo Dee.

Dry Branch

$-0.41993$

$-0.54318$

$-0.33598$

$-0.64741$

$-0.99369$
Congaree

0.419933

0.000000

$-0.12324$

0.083956

$-0.22748$

$-0.57375$
Blck Creek

0.123243
0.000000

0.000000

0.207198

$-0.10423$

$-0.45051$
McBean Tobacco Rd Middendorf

- 0.335978

0.227475

0.104232

0.311431

0.000000

$-0.34628$
Pee Dee

0.993687

0.573754

0.450511

0.657709

0.346279

0.000000
$-0.46585$

.0 .04592

0.069077

Congaree

$-0.20194$

0.227494

0.510249
$-0.04592$

$-0.46585$

$-0.35086$

Blck Creek 0.069077

$-0.35086$

$-0.4822$

$-0.45397$

$-0.19244$

0.090315
$-0.33788$

$-0.32481$
$-0.04088$
McBean Tobacco Rd $-0.20194$

$-0.45397$

$-0.33788$

$-0.60141$

$-0.18724$

0.104489
Middendorf 0.227494

$-0.19244$

$-0.32481$

$-0.18724$

$-0.36829$

$-0.09306$
Pee Dee

0.510249

0.090315

$-0.04088$

0.104489

$-0.09306$

$-0.50041$

Poslitivo values show pairs of means that are significantly different. 


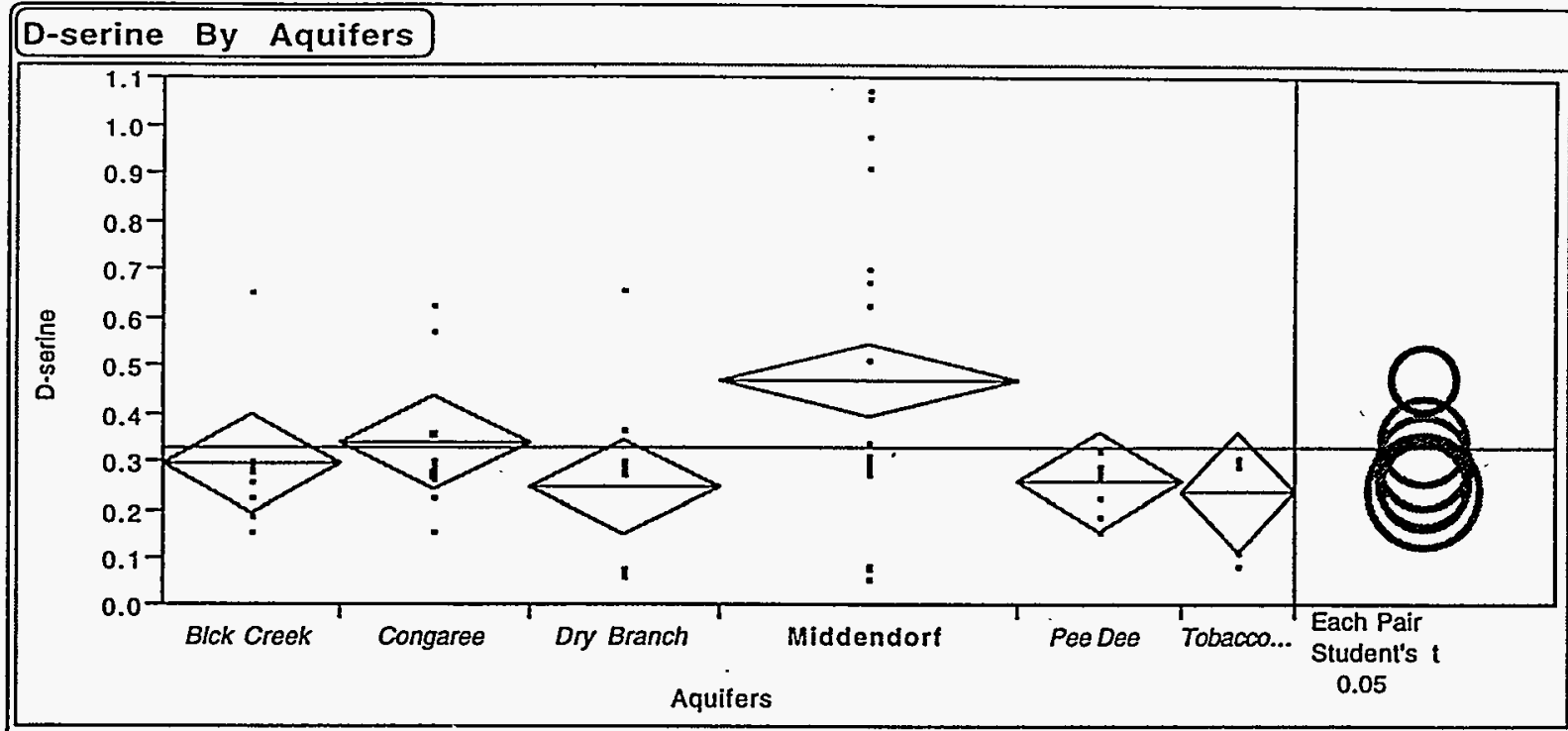

\section{Oneway Anova}

Summary of Fit

RSquare

RSquare Adj

Root Mean Square Error

Mean of Response

Observations (or Sum Wgts)

0.189569

0.141329

0.195038

0.327822

90

\begin{tabular}{|lrrrr|}
\hline \hline Analysis & of & Variance & & \\
Source & DF & Sum of Squares & Mean Square & F Ratio \\
Model & 5 & 0.7474269 & 0.149485 & 3.9297 \\
Error & 84 & 3.1953503 & 0.038040 & Prob $>$ F \\
C Total & 89 & 3.9427772 & & 0.0030 \\
\hline
\end{tabular}

Means for Oneway Anova

Level Number Mean Std Error

$\begin{array}{llll}\text { Blck Creek } & 14 & 0.293214 & 0.05213\end{array}$

$\begin{array}{llll}\text { Congaree } & 15 & 0.337333\end{array}$

$\begin{array}{lrll}\text { Dry Branch } & 15 & 0.244533 & 0.05036\end{array}$

McBean

$\begin{array}{llll}\text { Middendorf } & 24 & 0.468792 & 0.03981\end{array}$

$\begin{array}{llll}\text { Pee Dee } & 13 & 0.254923 & 0.05409\end{array}$

$\begin{array}{llll}\text { Tobacco Rd } & 9 & 0.234000 & 0.06501\end{array}$

Std Error uses a pooled estimate of error variance

Means Comparisons

Dif=Mean[i]-Mean[j] Middendorf Blck Creek

Middendorf

0.000000

0.175577

$-0.17558$

0.000000

Blck Creek

$-0.21387$

McBean

Congaree

$-0.13146$

$-0.03829$

0.213869

0.038291

McBean Congaree

- 0.1314580 .224258

Dry Branch Tobacco Rd

0.234792

- $\quad-0.04412$

0.048681

0.059214

$-0.08241$

0.044119

0.082410

$-0.22426$

$-0.04868$

0.082410

0.010390

0.020923

Dry Branch

$-0.23479$

$\begin{array}{ll}-0.05921 & -0.02092\end{array}$

- 0.000000

. $\quad-0.0928$

0.092800

0.103333

0.000000

0.010533

0.000000

Alpha $=0.05$

Comparisons for each pair using Student's $t$

1.98862

Abs(DIf)-LSD

Middendorf

Blck Creek

Pee Dee

McBean

Congaree

Dry Branch

Tobacco Rd

Middendorf
-0.11196

0.045143

0.080303

0.003799

0.096599

0.083191
McBean Congaree

Creek

$045143 \quad 0.080303$

$-0.1466$

$-0.1111$

$-0.10001$

$-0.09545$

$-0.1065$

$-0.14726$
- 0.003799

- -0.10001

- 0.06456

- 0.

- $\quad 0.14163$

- -0.04883

- 0.0602
Dry Branch Tobacco Rd

0.096599

$-0.09545$

$-0.13658$

$-0.04883$

$-0.14163$

$-0.153$
0.083191

$-0.1065$

$-0.14726$

$-0.0602$

$-0.153$

$-0.18284$

Positive values show pairs of means that are significantly different. 
FIGURE $16 \mathrm{q}$.

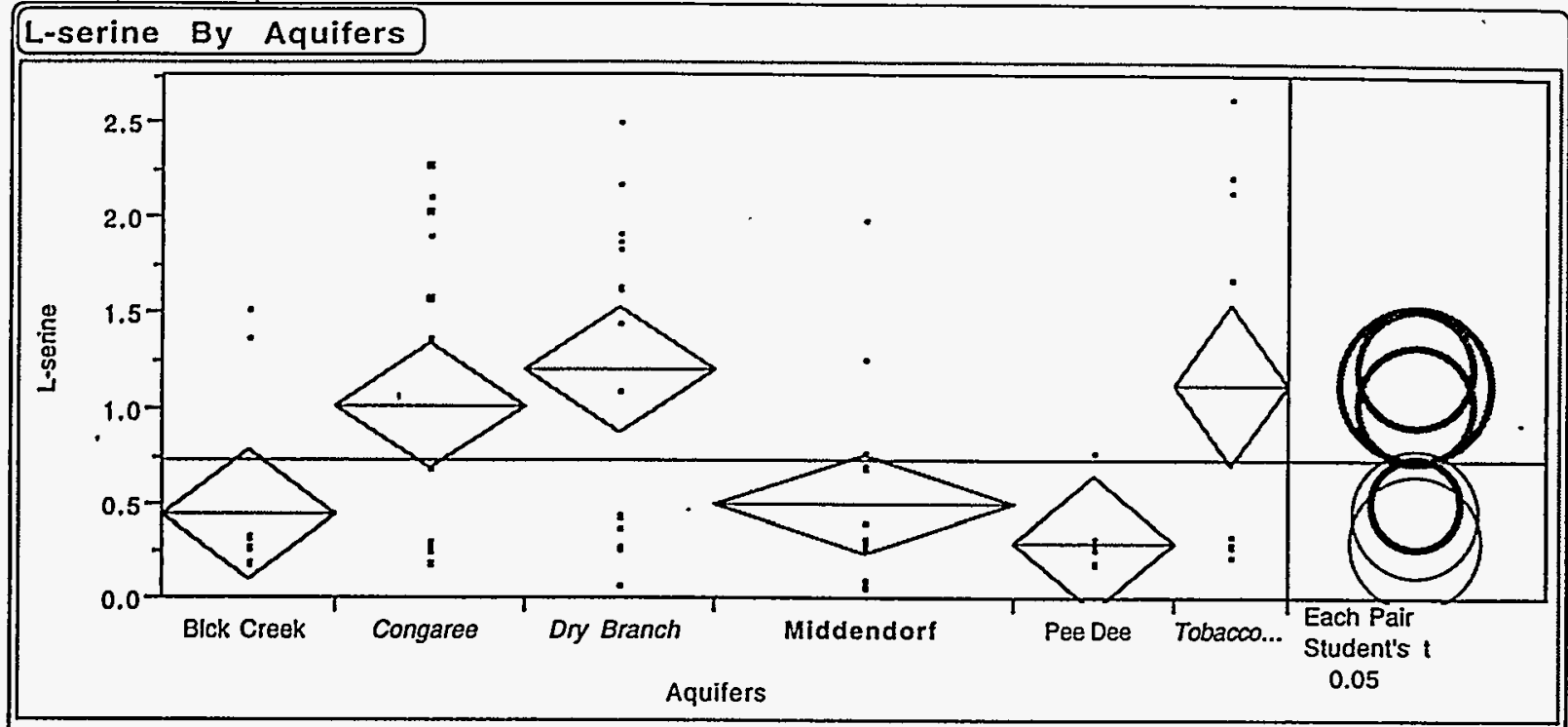

\section{Oneway Anova}

\begin{tabular}{|lr|}
\hline Summary of Fit & \\
RSquare & 0.229582 \\
RSquare Adj & 0.183724 \\
Root Mean Square Error & 0.653285 \\
Mean of Response & 0.722478 \\
Observatlons (or Sum Wgts) & 90 \\
\hline
\end{tabular}

\begin{tabular}{|lrrrrr|}
\hline Analysis & of & Variance & & \\
Source & DF & Sum of Squares & Mean Square & F Ratio \\
Model & 5 & 10.683078 & 2.13662 & 5.0064 \\
Error & 84 & 35.849596 & 0.42678 & Prob $>$ F \\
C Total & 89 & 46.532674 & & 0.0005 \\
\hline
\end{tabular}

Means for Oneway Anova

Lovel Number Mean Std Error

$\begin{array}{llll}\text { Blck Creak } & 14 & 0.43671 & 0.17460\end{array}$

$\begin{array}{llll}\text { Congaree } & 15 & 1.00380 & 0.16868\end{array}$

$\begin{array}{llll}\text { Dry Branch } & 15 & 1.19647 & 0.16868\end{array}$

McBean

$\begin{array}{llll}\text { Mlddendorf } & 24 & 0.50017 & 0.13335\end{array}$

$\begin{array}{llll}\text { Peo Dee } & 13 & 0.29454 & 0.18119\end{array}$

$\begin{array}{lrll}\text { Tobacco Rd } & 9 & 1.11911 & 0.21776\end{array}$

Std Error uses a pooled estimate of error variance

\section{Means Comparisons}

\section{DIf=Mean[I]-Mean[I] Dry Branch}

Dry Branch

Congaree

Blck Creek

McBean

Tobacco Rd

Middendorf

Peo Dee

Alpha $=0.05$

Comparisons for each pair using Student's $t$
Congaree Blck Creek

$\begin{array}{ll}-0.19267 & 0.000000\end{array}$

$-0.75975$

$-0.07736$

$-0.6963$

$-0.90193$
0.759752

0.567086

0.000000

0.682397

0.063452

$-0.14218$
McBean Tobacco Rd Middendorf 0.077356

$-0.11531$

$-0.6824$

0.000000

$-0.61894$

$-0.82457$
Pee Dee

$0.696300 \quad 0.901928$

$0.503633 \quad 0.709262$

$.0 .06345 \quad 0.142176$

$0.618944 \quad 0.824573$

$0.000000 \quad 0.205628$

$\begin{array}{ll}-0.20563 & 0.000000\end{array}$
1.98862

Abs(DIf)-LSD

Dry Branch

Congaree

Blck Creek

McBean

Tobacco Rd

Mlddendorf

Pee Dee

$\begin{array}{rrr}\text { Dry Branch } & \text { Congaree } & \text { Blck Creek } \\ -0.47438 & -0.28171 & 0.276979 \\ -0.28171 & -0.47438 & 0.084312 \\ 0.276979 & 0.084312 & -0.49103 \\ -0.47041 & -0.43245 & 0.127346 \\ 0.268703 & 0.076036 & -0.37344 \\ 0.409644 & 0.216978 & -0.3582\end{array}$

McBean Tobacco Rd Middendorf

$-0.47041$

$-0.43245$

0.127346

$-0.61242$

0.111155

0.261230 $\begin{array}{rr}\text { Middendorf } & \text { Pee Dee } \\ 0.268703 & 0.409644 \\ 0.076036 & 0.216978 \\ -0.37344 & -0.3582 \\ \cdot & \cdot \\ 0.111155 & 0.261230 \\ -0.37503 & -0.24175 \\ -0.24175 & -0.50956\end{array}$

Positive values show pairs of means that are signiticantly different. 
FIGURE $16 r$.

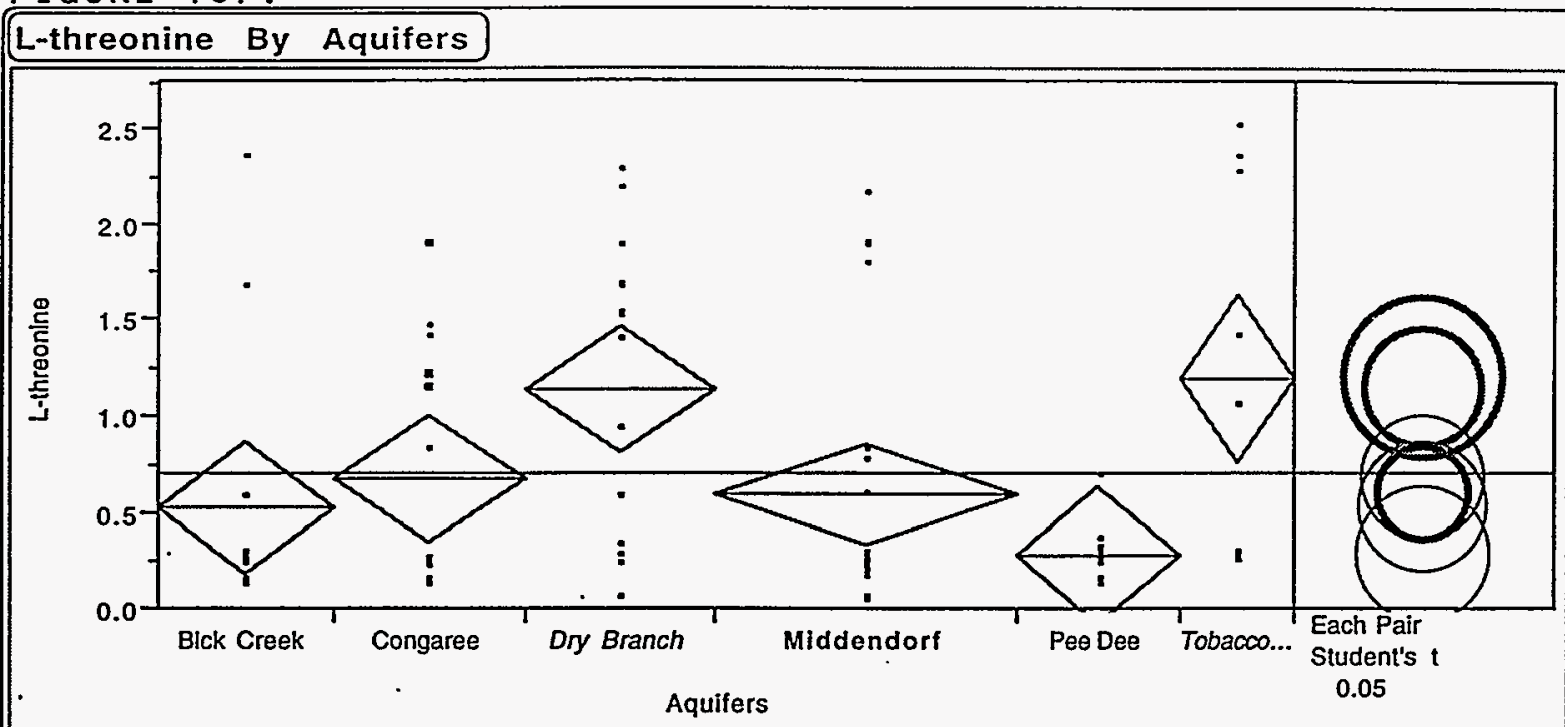

\section{Oneway Anova}

\section{Summary of Fit}

RSquare

RSquare Adi

0.180547

0.13177

Root Mean Square Error

Mean of Response

0.662035

Observations (or Sum Wgts)

0.699178

Analysis of Variance

\begin{tabular}{|lrrrrr} 
Source & DF & Sum of Squares & Mean Square & F Ratio \\
Model & 5 & 8.111643 & 1.62233 & 3.7015 \\
Error & 84 & 36.816434 & 0.43829 & Prob $>$ F \\
C Total & 89 & 44.928077 & & 0.0045 \\
\hline
\end{tabular}

Means for Oneway Anova

Level Number Mean Std Error

$\begin{array}{lrrr}\text { Blck Creek } & 14 & 0.51864 & 0.17694\end{array}$

$\begin{array}{llll}\text { Congaree } & 15 & 0.67533 & 0.17094\end{array}$

$\begin{array}{llll}\text { Dry Branch } & 15 & 1.13460 & 0.17094\end{array}$

McBean

Middendorf

Pee Dee

Tobacco Rd

0.58604

0.28238

1.19778

0.13514

0.18362

0.22068

Std Error uses a pooled estimate of error variance

\section{Means Comparisons}

Dif=Mean[i]-Mean[i] Dry Branch Congare

Dry Branch

Congaree

Blck Creek

McBean

Tobacco Rd

Middendorf

Pee Dee

Alpha $=0.05$

Comparisons for each pair using Student's $t$
0.000000

$-0.45927$

$-0.61596$

0.063178

$-0.54856$

$-0.85222$
0.459267

0.000000

$-0.15669$

0.522444

$-0.08929$

$-0.39295$

\subsection{7}

0.156690

0.000000

0.679135

0.067399

$-0.23626$
McBean Tobacco Rd Middendorf

$0.089292 \quad 0.392949$

$\begin{array}{ll}-0.0674 & 0.236258\end{array}$

0.000000

$-0.30366$
1.98862

Abs(Dif)-LS

Dry Branch

Congaree

Blck Creek

McBean

Tobacco Rd

Middendorf

Pee Dee

Dry Branch

$-0.48073$

$-0.02146$

0.126717

Congaree

$-0.02146$

$-0.48073$

$-0.33255$

$-0.49192$

0.115233

0.353337

$-0.03266$

-0.3440 '

$-0.10593$
Blck Creek

0.126717

$-0.33255$

$-0.4976$

0.116650

$-0.37535$

$-0.27082$
McBean Tobacco Rd

$-0.49192$

$-0.03266$

0.116650

$-0.62062$

0.097145

0.344505
Middendorf

0.115233

$-0.34403$

$-0.37535$

0.097145

$-0.38005$

$-0.14972$
Pee Dee

0.353337

$-0.10593$

$-0.27082$

0.344505

$-0.14972$

$-0.51639$

Positive values show pairs of means that are significantly different. 
FIGURE $16 \mathrm{~s}$.

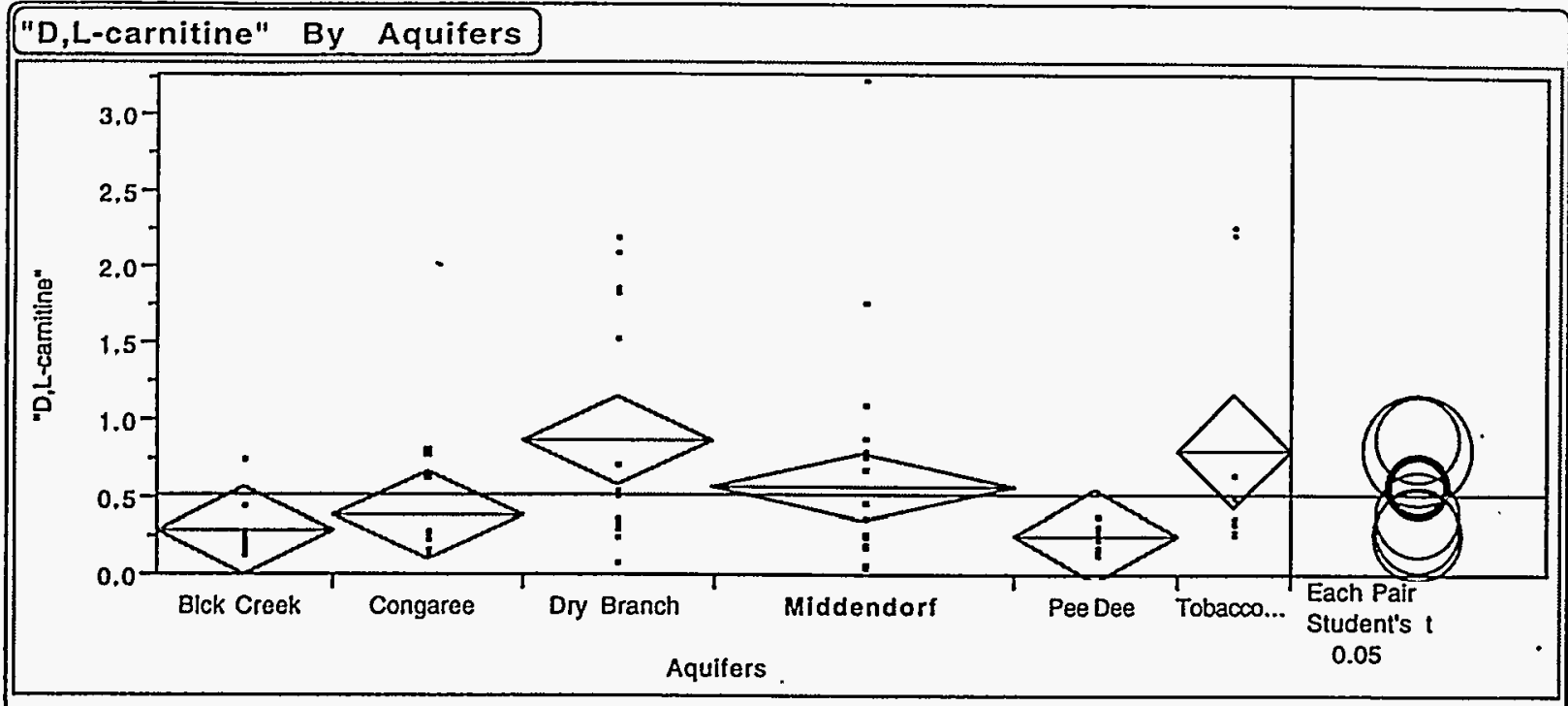

Oneway Anova

Summary of Fit

RSquare

RSquare AdI

Rool Mean Square Error

Mean of Response

Observations (or Sum Wgts)

0.097284

0.55574

0.528967

\begin{tabular}{|lrrrrr|}
\hline Analysis & of & Variance & & \\
Source & DF & Sum of Squares & Mean Square & F Ratio \\
Model & 5 & 4.506503 & 0.901301 & 2.9183 \\
Error & 84 & 25.943156 & 0.308847 & Prob $>$ F \\
C Total & 89 & 30.449659 & & 0.0177 \\
\hline
\end{tabular}

\begin{tabular}{|lrrrr|}
\hline \multicolumn{4}{|l}{ Means for Oneway } & \multicolumn{3}{c|}{ Anova } \\
\cline { 2 - 4 } Level & Number & Mean & Std Error \\
Blck Creek & 14 & 0.296786 & 0.14853 \\
Congaree & 15 & 0.383000 & 0.14349 \\
Dry Branch & 15 & 0.876000 & 0.14349 \\
McBean & 0 &. &. \\
Middendor & 24 & 0.580458 & 0.11344 \\
Pee Dee & 13 & 0.265000 & 0.15413 \\
Tobacco Rd & 9 & 0.799000 & 0.18525 \\
Std Error uses a pooled estimate of error variance &
\end{tabular}

\section{Means Comparisons}

$\begin{array}{lrrr}\text { DIf=Mean[I]-Mean[]] } & \text { Dry Branch } & \text { Middendorf } & \text { Blck Creek } \\ \text { Dry Branch } & 0.000000 & 0.295542 & 0.579214 \\ \text { Middendorf } & -0.29554 & 0.000000 & 0.283673 \\ \text { Blck Creek } & -0.57921 & -0.28367 & 0.000000 \\ \text { McBean } & - & - & \\ \text { Tobacco Rd } & -0.077 & 0.218542 & 0.502214 \\ \text { Congaree } & -0.493 & -0.19746 & 0.086214 \\ \text { Pee Dee } & -0.611 & -0.31546 & -0.03179\end{array}$

$\begin{array}{rrrr}\text { McBean } & \text { Tobacco Rd } & \text { Congaree } & \text { Pee Dee } \\ \bullet & 0.077000 & 0.493000 & 0.611000 \\ - & -0.21854 & 0.197458 & 0.315458 \\ - & -0.50221 & -0.08621 & 0.031786 \\ - & 0.000000 & 0.416000 & 0.534000 \\ - & -0.416 & 0.000000 & 0.118000 \\ - & -0.534 & -0.118 & 0.000000\end{array}$

Alpha $=0.05$

Comparisons for each pair using Student's $t$

$t$

1.98862

Abs(Dif)-LSD Dry Branch Middendorf Blck Creek

Dry Branch

Middendort

$-0.40355$

$-0.06821$

$-0.31903$

0.168526

$-0.08799$

McBean

Tobacco Rd

Congaree

$-0.38897$

0.089455

$-0.21343$

$-0.16629$

$-0.06512$
McBean Tobacco Rd

$-0.38897$

$-0.21343$

0.030041

$-0.52097$

$-0.04997$

0.054773
Congaree

0.089455

$-0.16629$

$-0.32447$

$-0.04997$

$-0.40355$

$-0.30078$
Pee Dee

0.192221

$-0.06512$

$-0.39388$

0.054773

$-0.30078$

$-0.43348$

Posiltve values show pairs of means that are significantly different. 
FIGURE $16 t$.

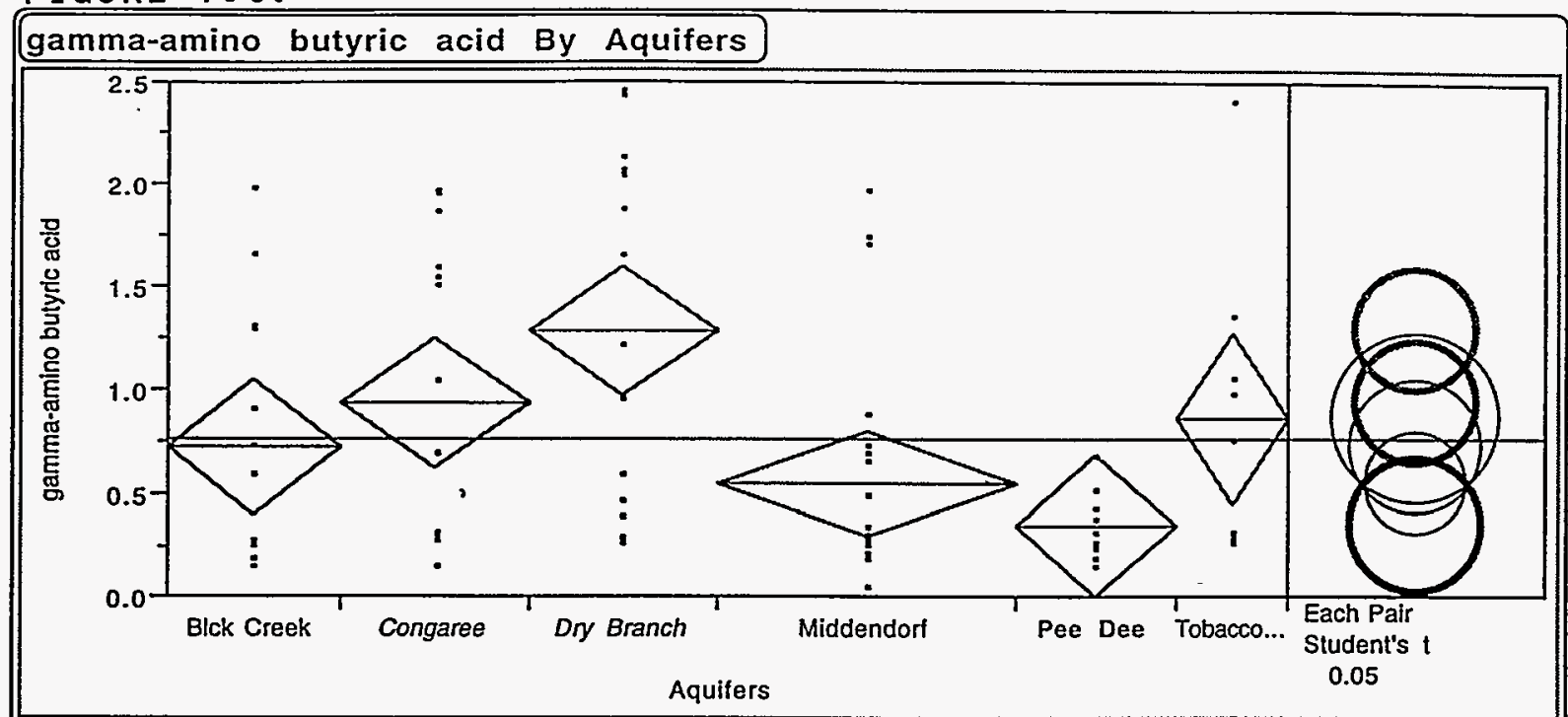

\section{Oneway Anova}

\section{Summary of Fit}

RSquare

0.193427

RSquare Adj

0.145417

0.633132

Root Mean Square Error

Mean of Response

0.763933

Observations (or Sum Wgts)

90

\begin{tabular}{|lrrrrr|}
\hline Analysis & of & Variance & & \\
Source & DF & Sum of Squares & Mean Square & F Ratio \\
Model & 5 & 8.074989 & 1.61500 & 4.0289 \\
Error & 84 & 33.671930 & 0.40086 & Prob $>$ F \\
C Total & 89 & 41.746920 & & 0.0025 \\
\hline
\end{tabular}

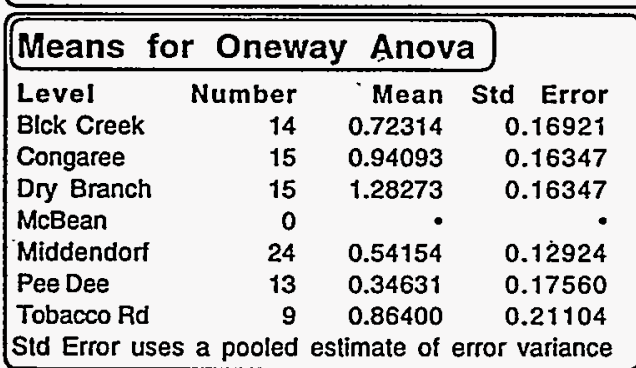

Means Comparisons

Dif=Mean[i]-Mean[j] Dry Branch Congaree Blck Creek

Dry Branch

Congaree

Blck Creek

0.000000

0.341800

0.559590

McBean Tobacco Rd Middendorf

Pee Dee

$-0.3418 \quad 0.000000$

0.217790
0.000000

McBean

Tobacco Rd

$-0.55959$

$-0.21779$

$-0.41873-0.07693$

Middendorf

$-0.74119-0.39939$

$-0.93643-0.59463$

0.140857

$-0.1816$

$-0.37684$

0.418733
- $\quad 0.076933$

$0.741192 \quad 0.936426$

$\begin{array}{ll}0.741192 & 0.936426 \\ 0.399392 & 0.594626\end{array}$

$-0.14086$

0.181601

0.376835

Pee Dee

- 0.000000

0.322458

0.517692

0.000000

0.195234

$\begin{array}{ll}- & -0.32246 \\ -\quad & -0.51769\end{array}$

$-0.19523$

0.000000

Comparisons for each pair using Student's $t$ 1.98862

Abs(Dif)-LSD Dry Branch Congaree

Blck Creek

McBean

Tobacco Rd

Middendorf

Pee Dee
Dry Branch Congaree Blck Creek
$-0.45974-0.11794$
$-0.11794$
0.091710
$-0.47588$
$-0.11213-0.45393$
0.326785
$-0.01502$
$-0.39707$
$-0.39707$
$-0.24182$

McBean Tobacco Rd Middendorf

$-0.10811$

$-0.11213$

Pee Dee

$-0.45393$

. $\quad-0.39707$

$-0.01502$

$-0.24182$

$-0.59353$

$-0.16967$

$-0.16967$

$-0.36346$

$-0.23835$

.0 .10811

Positive values show pairs of means that are significantly different. 
FIGURE 17.

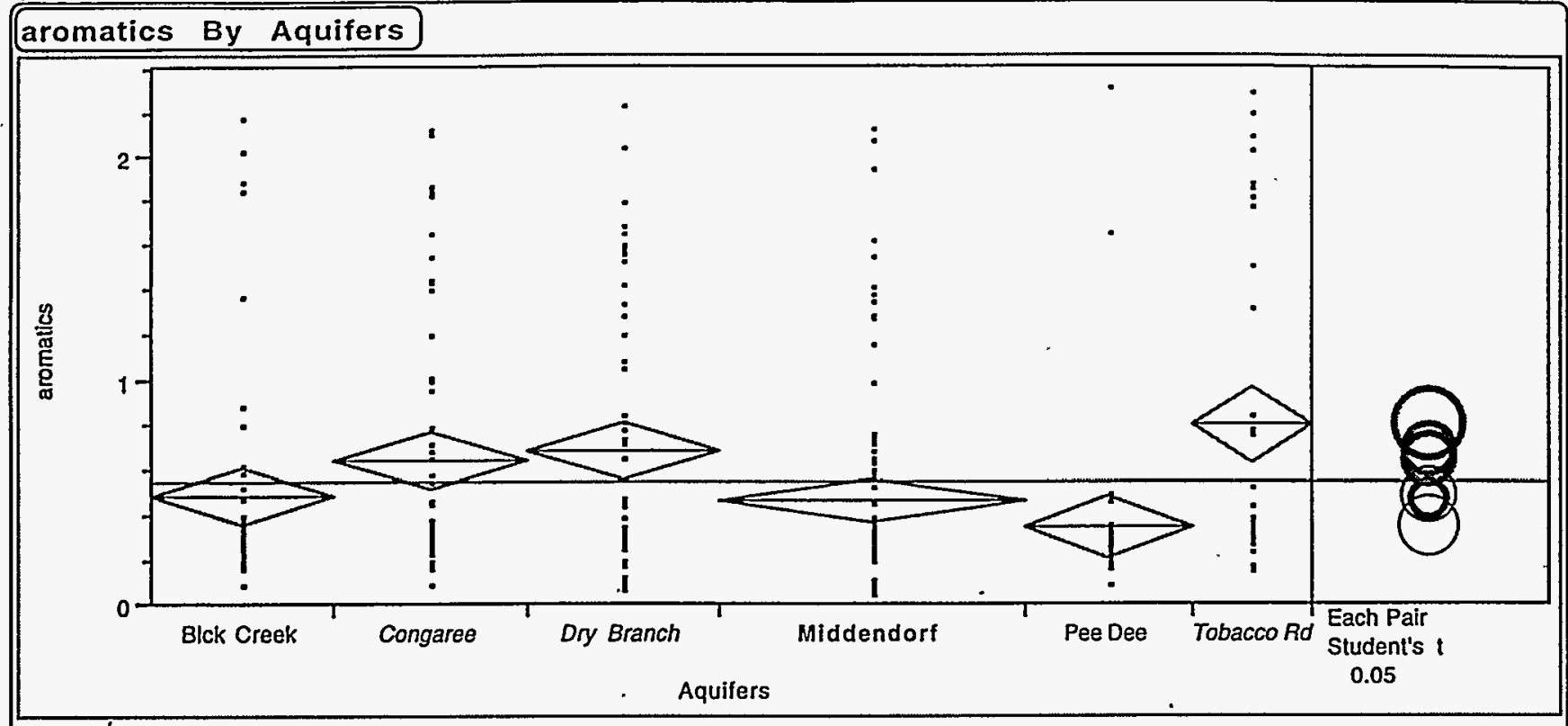

\section{Oneway Anova}

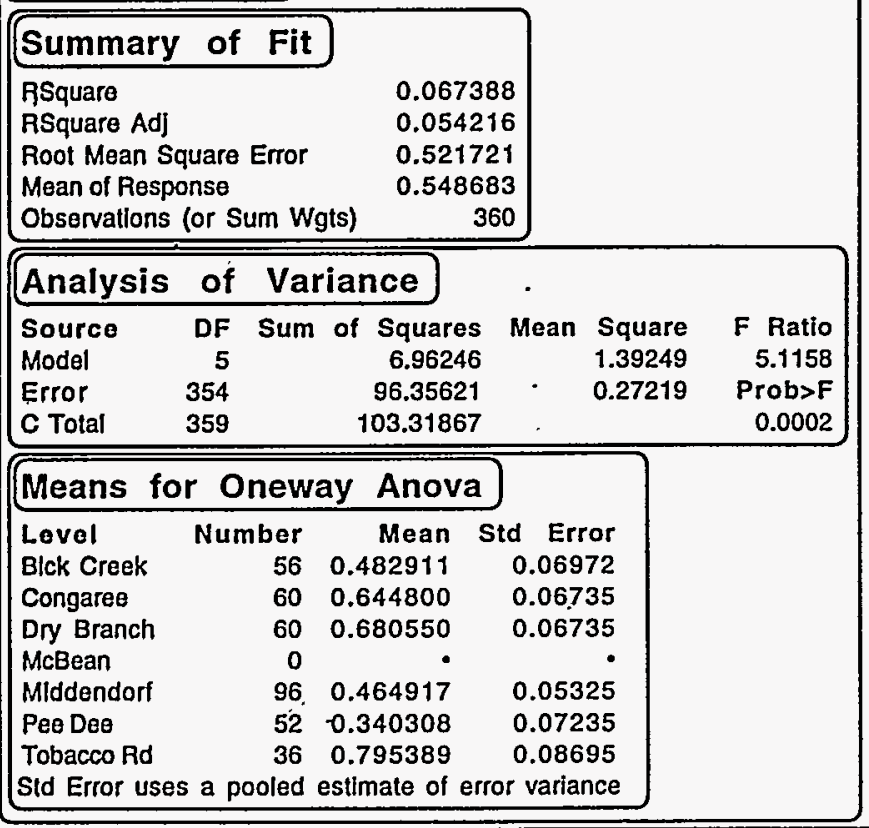

\section{Means Comparisons}

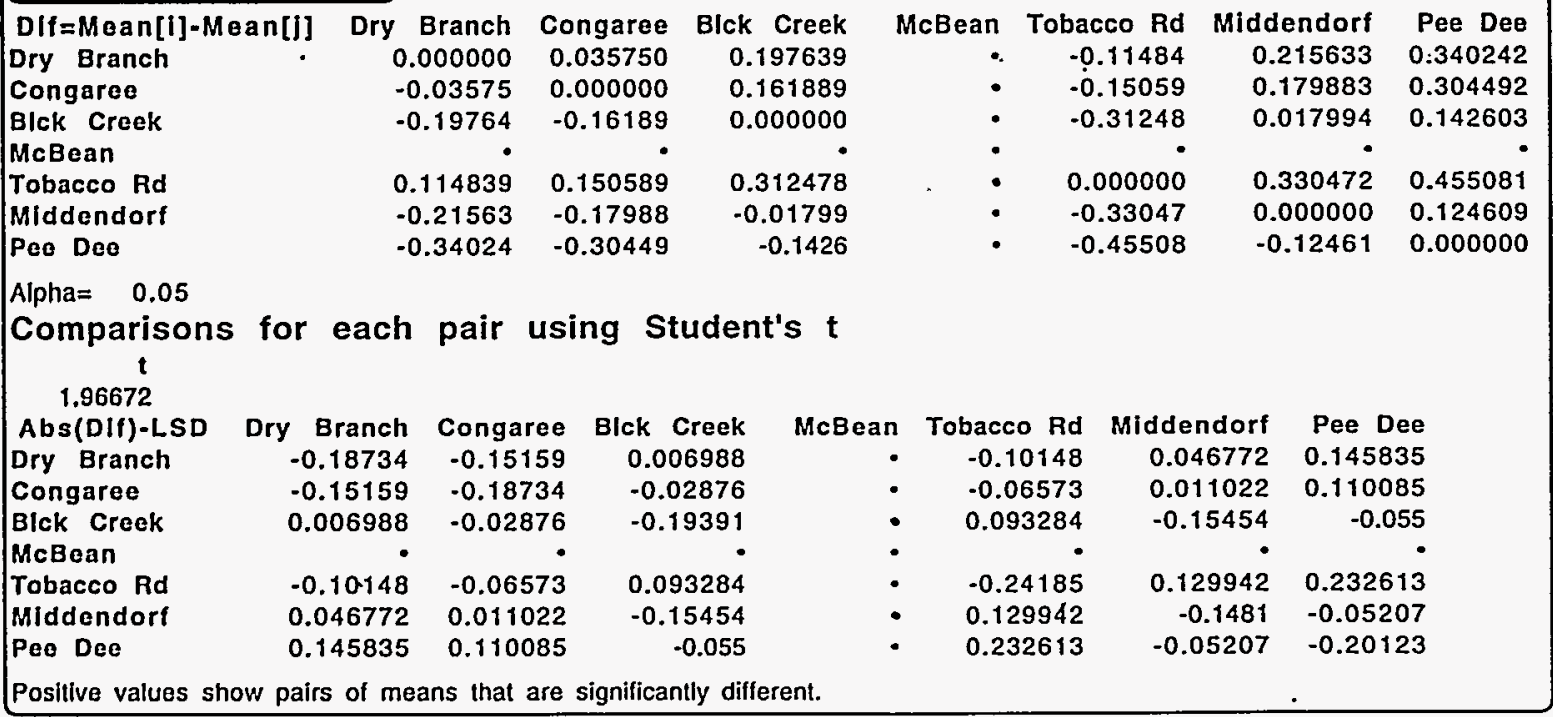


FIGURE $17 \mathrm{a}$.

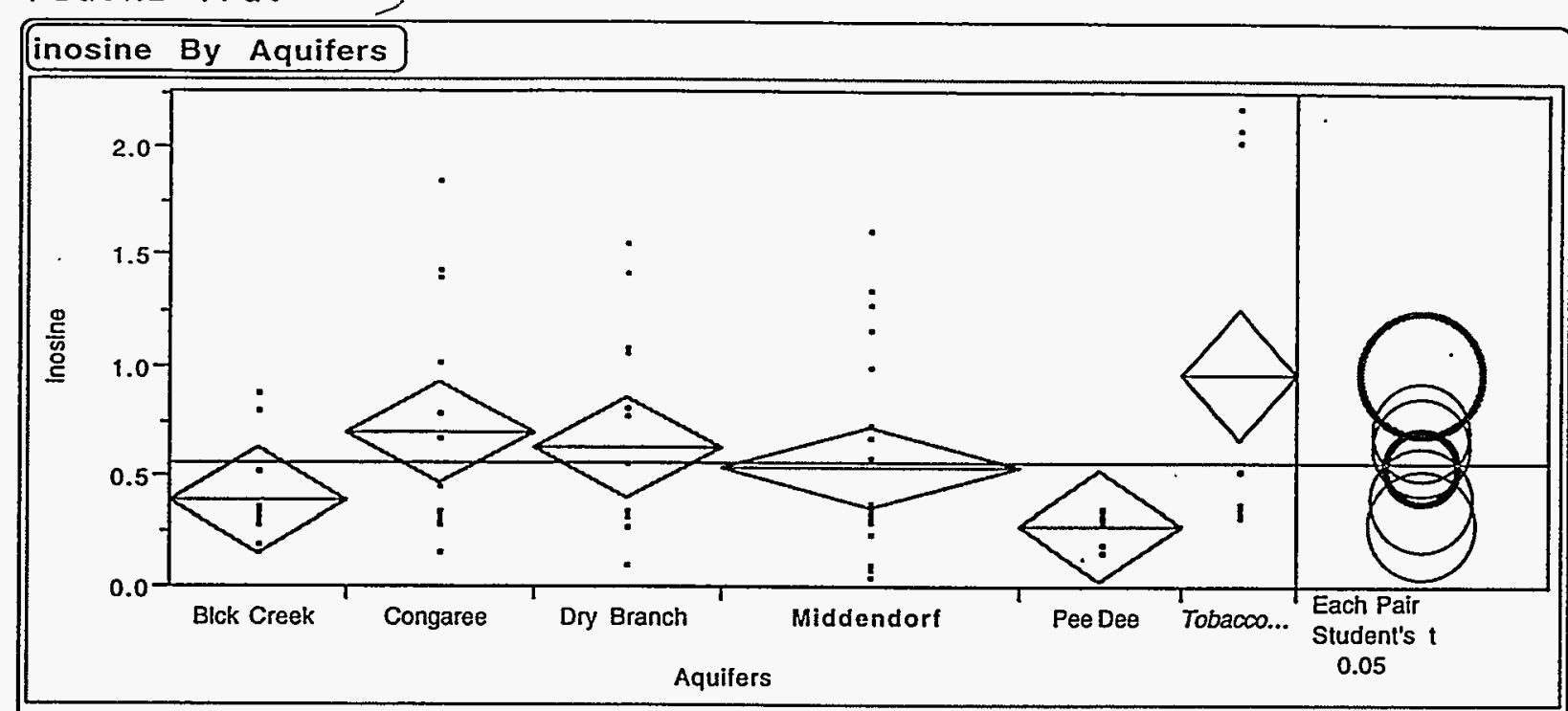

\section{Oneway Anova}

\section{Summary of Fit}

RSquare

RSquare Adj

Root Mean Square Error

Mean of Response

Observations (or Sum Wgts)
0.153707

0.103332

0.460595

0.562789

\section{Analysis of Variance}

\begin{tabular}{|lrrrr} 
Source & DF & Sum of Squares & Mean Square & F Ratio \\
Model & 5 & 3.236611 & 0.647322 & 3.0513 \\
Error & 84 & 17.820438 & 0.212148 & Prob>F \\
C Total & 89 & 21.057049 & & 0.0140 \\
\hline
\end{tabular}

Means for Oneway Anova

Level Number Méan Std Error

$\begin{array}{llll}\text { Blck Creek } & 14 & 0.390357 & 0.12310\end{array}$

$\begin{array}{llll}\text { Congaree } & 15 & 0.704467 & 0.11893\end{array}$

$\begin{array}{llll}\text { Dry Branch } & 15 & 0.628867 & 0.11893\end{array}$

McBean

Middendorf

Pee Dee

Tobacco Rd

$\begin{array}{lll}24 & 0.537708 & 0.09402\end{array}$

240.53

$13 \quad 0.281231$

90.958333

0.12775

0.15353

Std Error uses a pooled estimate of error variance

\section{Means Comparisons}

Dif=Mean[i]-Mean[j] Congaree Congaree

Dry Branch

Blck Creek

McBean

Tobacco Rd

Middendorf

Pee Dee

Congaree
0.000000
-0.0756
-0.31411
0
0.253867
-0.16676
-0.42324

Dry Branch

0.075600

0.000000

$-0.23851$

0.329467

$-0.09116$

$-0.34764$
Blck Creek

0.314110

0.238510

0.000000

0.567976

0.147351

$-0.10913$
McBean Tobacco Rd Middendorf

0.166758

0.091158

$-0.14735$

0.420625

0.000000

$-0.25648$
Pee Dee

0.423236

0.347636

0.109126

0.677103

0.256478 0.000000

Alpha $=0.05$

Comparisons for each pair using Student's $t$

\section{t}

1.98862

Abs(Dif)-LSD Congaree

Congaree

Dry Branch

Blck Creek

McBean

Tobacco Rd

Middendorf

Pee Dee

$-0.33446$

$-0.25886$

$-0.02627$

$-0.13233$

$-0.13472$

0.076153
Dry Branch $-0.25886$

$-0.33446$

$-0.10187$

$-0.05673$

$-0.21032$

0.000553
Blck Creek $-0.02627$

$-0.10187$

$-0.3462$

0.176640

$-0.16068$

$-0.24366$
McBean Tobacco Rd Middendorf

$-0.13472 \quad 0.076153$

$\begin{array}{ll}-0.21032 & 0.000553\end{array}$

$\begin{array}{ll}-0.16068 & -0.24366\end{array}$

$0.176640 \quad-0.16068$

0.062610

$-0.26441-0.05895$

$-0.05895-0.35926$

Positive values show pairs of means that are significantly different. 
FIGUŔE $17 \mathrm{~b}$.

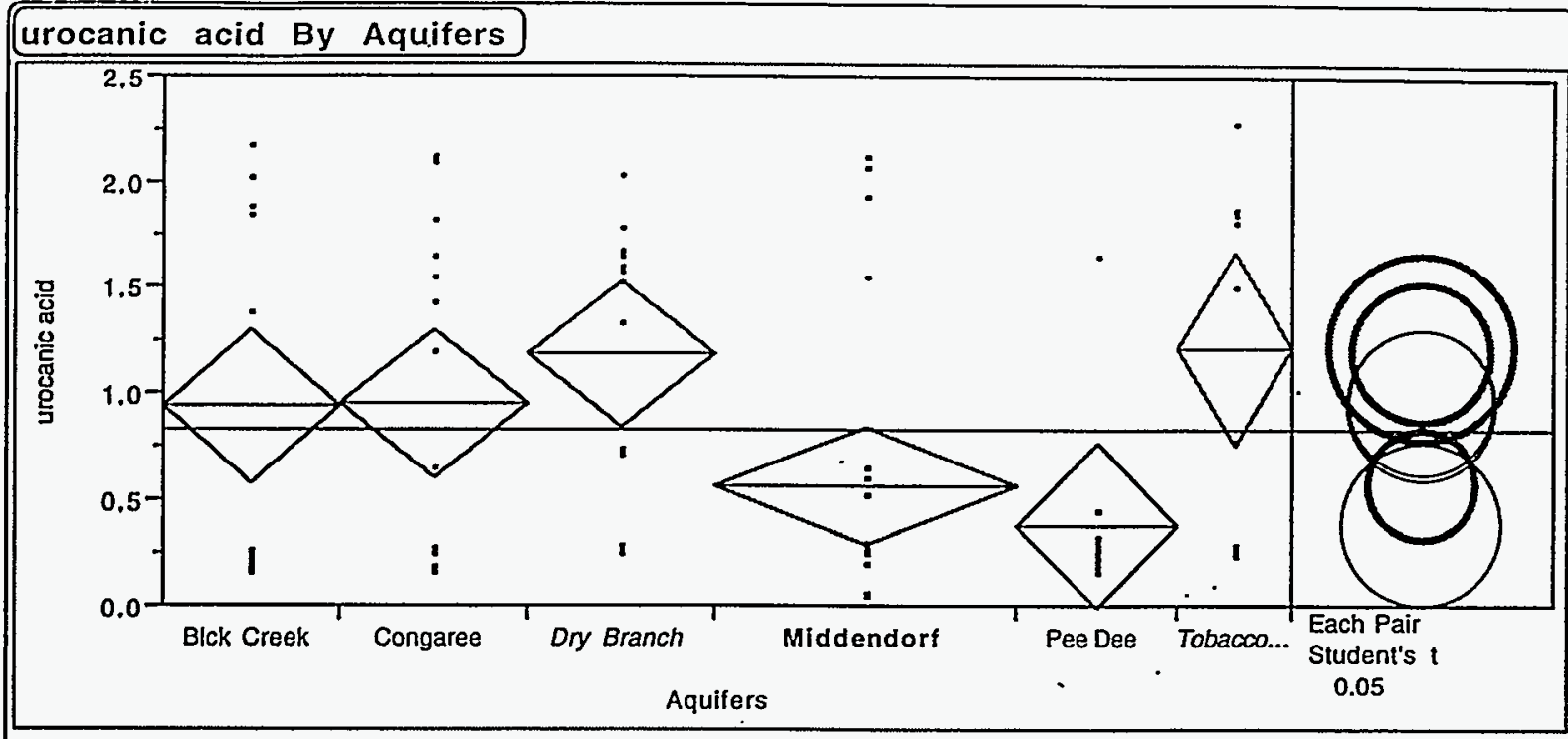

\section{Oneway Anova}

\begin{tabular}{|lr|}
\hline Summary of Fit & \\
RSquare & 0.162356 \\
RSquare Adj & 0.112497 \\
Root Mean Square Error & 0.696969 \\
Mean of Response & 0.8275 \\
Observatlons (or Sum Wgts) & 90 \\
\hline
\end{tabular}

\begin{tabular}{|lrrrrr|}
\hline Analysis & of & Variance & & \\
Source & DF & Sum of Squares & Mean Square & F Ratio \\
Model & 5 & 7.908918 & 1.58178 & 3.2563 \\
Error & 84 & 40.804367 & 0.48577 & Prob $>F$ \\
C Tolal & 89 & 48.713285 & & 0.0098 \\
\hline
\end{tabular}

\begin{tabular}{|c|c|c|c|}
\hline \multicolumn{4}{|c|}{ Means for Oneway Anova } \\
\hline $\begin{array}{l}\text { Level } \\
\text { Blck Creek }\end{array}$ & $\begin{array}{r}\text { Number } \\
14\end{array}$ & $\begin{array}{r}\text { Mean } \\
0.93271\end{array}$ & $\begin{array}{l}\text { Std Error } \\
0.18627\end{array}$ \\
\hline Congaree & 15 & 0.94827 & 0.17996 \\
\hline Dry Branch & 15 & 1.18567 & 0.17996 \\
\hline McBean & 0 & - & - \\
\hline Middendorf & 24 & 0.56629 & 0.14227 \\
\hline Pee Dee & 13 & 0.37708 & 0.19330 \\
\hline Tobacco Rd & 9 & 1.21278 & 0.23232 \\
\hline
\end{tabular}

Means Comparisons

$\begin{array}{lrrrrrrr}\text { Dif=Mean[i]-Mean[j] } & \text { Dry Branch } & \text { Congaree } & \text { Blck Creek } & \text { McBean } & \text { Tobacco Rd } & \text { Middendorf } & \text { Pee Dee } \\ \text { Dry Branch } & 0.000000 & 0.237400 & 0.252952 & & -0.02711 & 0.619375 & 0.808590 \\ \text { Congaree } & -0.2374 & 0.000000 & 0.015552 & & -0.26451 & 0.381975 & 0.571190 \\ \text { Blck Creek } & -0.25295 & -0.01555 & 0.000000 & & -0.28006 & 0.366423 & 0.555637 \\ \text { McBean } & - & - & - & & & \\ \text { Tobacco Rd } & 0.027111 & 0.264511 & 0.280063 & & 0.000000 & 0.646486 & 0.835701 \\ \text { Middendorf } & -0.61937 & -0.38197 & -0.36642 & & -0.64649 & 0.000000 & 0.189215 \\ \text { Pee Deo } & -0.80859 & -0.57119 & -0.55564 & - & -0.8357 & -0.18921 & 0.000000\end{array}$

Alpha $=0.05$

Comparisons for each pair using Student's $t$

1.98862

Abs(Dif)-Ls

Dry Branch

Congareo

Blck Creek

Dry Branch

$$
\begin{aligned}
& -0.5061 \\
& -0.2687 \\
& -0.2621
\end{aligned}
$$
Congaree Blck Creek 0.2687
$-0.5061$
$-0.4995$
$-0.2621$
$-0.4995$
$-0.52386$
$-0.3121$
$-0.09969$

McBean

Tobacco Rd

Middendorf

Poo Dee

$\begin{array}{ll}-0.55728 & -0.31988\end{array}$

$0.163185-0.07422$

0.2833870 .045987

0.021797
McBean Tobacco Rd
-0.55728
-0.31988

Middendorf Pee Dee

$\begin{array}{lll}0.163185 & 0.283387\end{array}$

$\begin{array}{ll}-0.07422 & 0.045987\end{array}$

$\begin{array}{lll}-0.3121 & -0.09969 & 0.021797\end{array}$

$\begin{array}{lll}-0.65337 & 0.104741 & 0.234688\end{array}$

$\begin{array}{lll}0.104741 & -0.40011 & -0.28808\end{array}$

Posilive values show pairs of means that are significantly different. 
FIGURE $17 \mathrm{C}$.

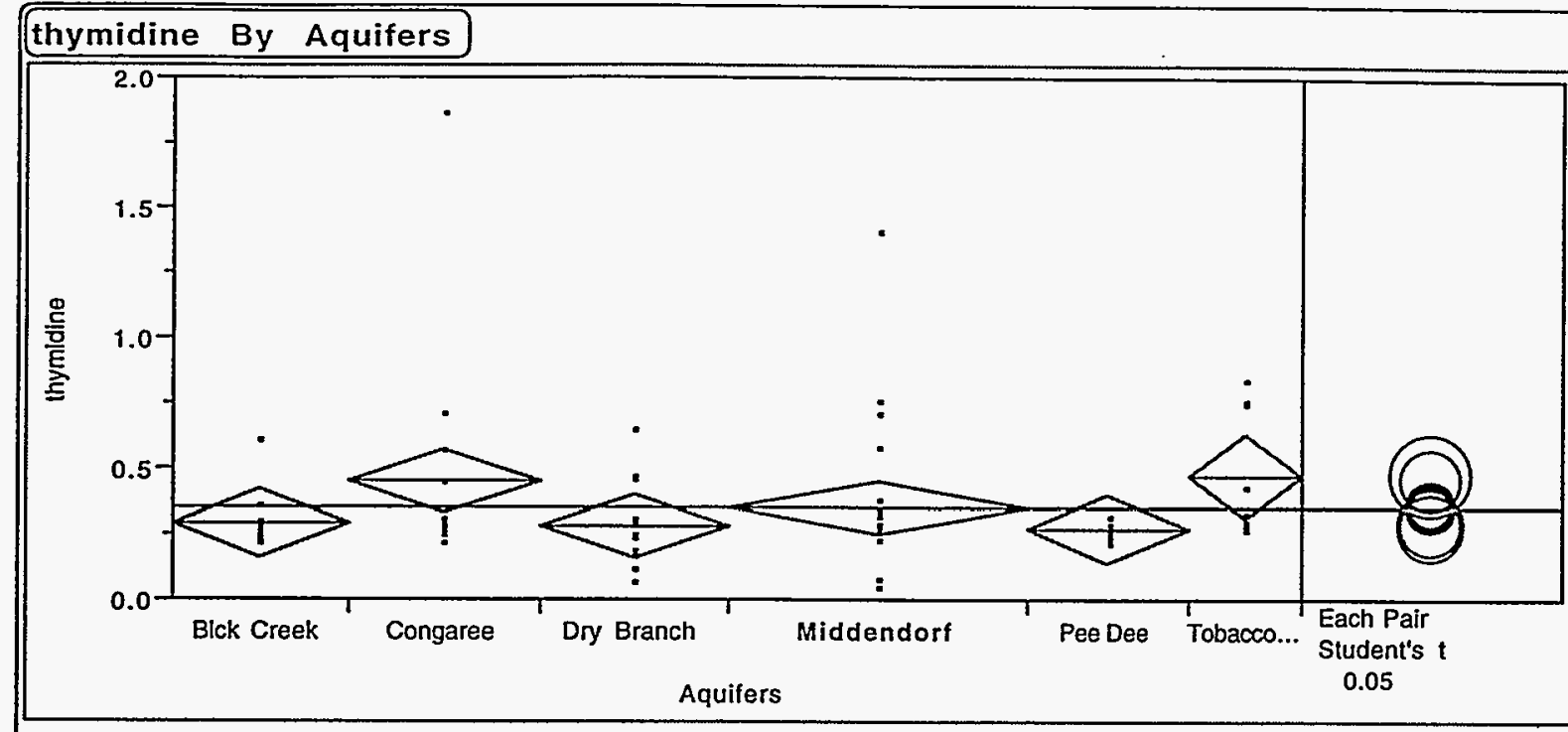

\section{Oneway Anova}

\section{Summary of Fit}

RSquare

6574

RSquare Adj

0.032203

Root Mean Square Error

Mean of Response

0.249008

Observations (or Sum Wgts)

0.349967

\begin{tabular}{|lrrrrr|}
\hline Analysis & of & Variance & & \\
Source & DF & Sum of Squares & Mean Square & F Ratio \\
Model & 5 & 0.4936515 & 0.098730 & 1.5923 \\
Error & 84 & 5.2084374 & 0.062005 & Prob>F \\
C Total & 89 & 5.7020889 & & 0.1711 \\
\hline
\end{tabular}

Means for Oneway Ariova

Level Number Mean Std Error

$\begin{array}{llll}\text { Blck Creek } & 14 & 0.295143 & 0.06655\end{array}$

$\begin{array}{llll}\text { Congaree } & 15 & 0.455333 & 0.06429\end{array}$

$\begin{array}{llll}\text { Dry Branch } & 15 & 0.283400 & 0.06429\end{array}$

McBean

Middendorf

Pee Dee

240.353000 " 0.05083

$\begin{array}{lll}9 & 0.474667 & 0.08300\end{array}$

Std Error uses a pooled estimate of error variance

\section{Means Comparisons}

Dif=Mean[i]-Mean[i] Congaree Congaree

Blck Creek

Dry Branch

McBean

Tobacco Rd

Middendorf

Pee Dee

0.000000

$-0.16019$

$-0.17193$

0.019333

$-0.10233$

$-0.18303$
Blck Creek

Dry Branch

0.000000

$-0.01174$

0.179524

0.057857

$-0.02284$

$$
\begin{array}{r}
0.171933 \\
0.011743 \\
0.000000 \\
0.191267 \\
0.069600 \\
-0.01109
\end{array}
$$

McBean Tobacco Rd Middendorf

0.183026

$\begin{array}{ll}-0.05786 & 0.022835\end{array}$

$-0.0696 \quad 0.011092$

$0.121667 \quad 0.202359$

$0.000000 \quad 0.080692$

$\begin{array}{ll}-0.08069 & 0.000000\end{array}$

Alpha $=0.05$

Comparisons for each pair using Student's t

$$
1
$$

1.98862

Abs(Dit)-LSD

Congaree

Blck Creek

Dry Branch

McBean

Tobacco Rd

Middendorf

Pee Dee

$$
\begin{array}{rrr}
\text { Congaree } & \text { Blck Creek } & \text { Dry Branch } \\
-0.18082 & -0.02383 & -0.00888 \\
-0.02383 & -0.18716 & -0.17227 \\
-0.00888 & -0.17227 & -0.18082 \\
\cdot & \bullet & - \\
-0.18945 & -0.03204 & -0.01752 \\
-0.06065 & -0.10867 & -0.09338 \\
-0.00462 & -0.16789 & -0.17655
\end{array}
$$

McBean Tobacco Rd Middendorf

- $\quad 0.18945$

$-0.06065$

$-0.10867$

$-0.09338$

Pee Dee

$-0.00462$

$-0.16789$

-0.03204
$-\quad 0.01752$

- $\quad-0.23343$

$-0.23343$

- $\quad-0.0718$

$-0.07188$

$-0.17655$

Positive values show pairs of means that are significantly different.

$-0.14295$

$-0.01237$

$-0.19423$ 
FIGURE 17d.

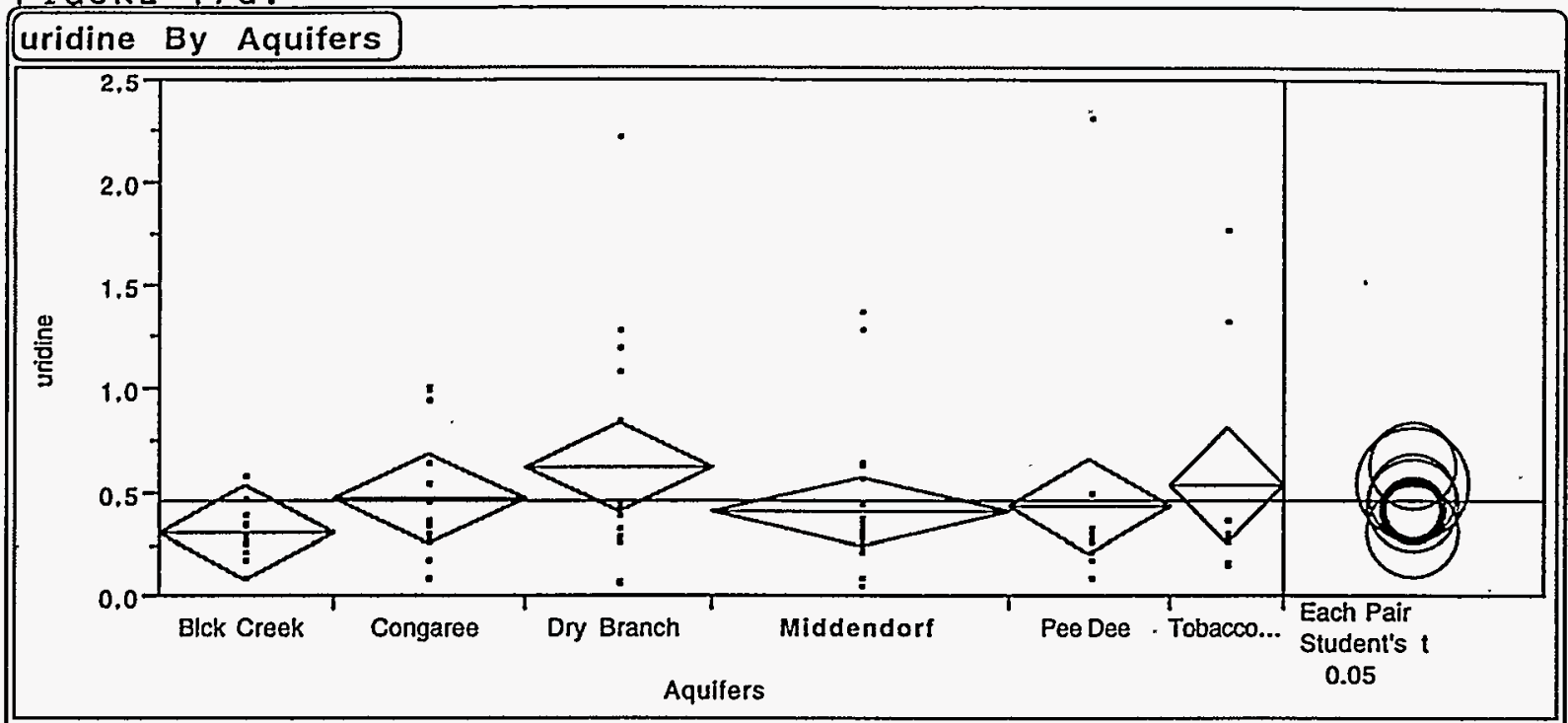

\begin{tabular}{|lr}
\hline Oneway Anova & \multicolumn{1}{|l}{} \\
\hline Summary of Fit & \\
\hline RSquare & 0.051363 \\
RSquare AdJ & -0.0051 \\
Root Mean Square Error & 0.431398 \\
Mean of Response & 0.454478 \\
Observatlons (or Sum Wgts) & 90 \\
\hline
\end{tabular}

\begin{tabular}{||lrrrr|}
\hline Analysis & of & Variance & & \\
Source & DF & Sum of Squares & Mean Square & F Ratio \\
Model & 5 & 0.846428 & 0.169286 & 0.9096 \\
Error & 84 & 15.632756 & 0.186104 & Prob>F \\
C Tolal & 89 & 16.479184 & & 0.4789 \\
\hline
\end{tabular}

\begin{tabular}{|lrrr|}
\hline Means for Oneway & Anova \\
Level & Number & Mean & Std Error \\
Blck Creek & 14 & 0.313429 & 0.11530 \\
Congaree & 15 & 0.471133 & 0.11139 \\
Dry Branch & 15 & 0.624267 & 0.11139 \\
McBean & 0 & & . \\
Middendorf & 24 & 0.402667 & 0.08806 \\
Pee Dee & 13 & 0.430615 & 0.11965 \\
Tobacco Rd & 9 & 0.535778 & 0.14380 \\
Std Error uses a pooled estimate of error variance &
\end{tabular}

\section{Means Comparisons}

Dif=Mean[i]-Mean[I] Dry Branch

Dry Branch

Congareo

Blck Creek

McBean

Tobacco Rd

Pee Dee

Mlddendorf

Alpha $=0,05$

Comparisons for each pair using Student's t 0.000000

$-0.15313$

$-0.08849$

$-0.2216$

$\begin{array}{rrr}\text { Branch } & \text { Congaree } & \text { Blck Creek } \\ 0.000000 & 0.153133 & 0.310838 \\ -0.15313 & 0.000000 & 0.157705 \\ -0.31084 & -0.1577 & 0.000000 \\ -0.08849 & 0.064644 & 0.222349 \\ -0.19365 & -0.04052 & 0.117187 \\ -0.2216 & -0.06847 & 0.089238\end{array}$

McBean Tobacco Rd 0.088489

$-0.06464$

$-0.22235$

0.000000

$-0.10516$

$-0.13311$
Pee Dee 0.193651 0.040518 $-0.11719$

0.105162 0.000000

$-0.02795$
Middendorf 0.221600 0.068467 $-0.08924$

0.133111 0.027949 0.000000
1.98862

Abs(DIf)-LSD

Dry Branch

Congaree

Blck Creek

McBean

Tobacco fid

Pee Deo

Middendorf

$$
\begin{array}{rr}
\text { Congaree } & \text { Blck Creek } \\
-0.16012 & -0.00796 \\
-0.31326 & -0.1611 \\
-0.1611 & -0.32425 \\
-0.29707 & -0.14418 \\
-0.28456 & -0.21324 \\
-0.2139 & -0.19927
\end{array}
$$

McBean Tobacco Rd

$-0.27323$

- $\quad-0.29707$

- -0.14418

-

$-0.40441$

$-0.26684$

$-0.20221$
Pee Dee Middendorf $-0.13143 \quad-0.06076$ $-0.28456 \quad-0.2139$ $-0.21324 \quad-0.19927$

$-0.26684 \quad-0.20221$

$-0.33649-0.26748$

$\begin{array}{ll}-0.26748 & -0.24765\end{array}$

Positive values show pairs of means that are significantly different. 
FIGURE 18.

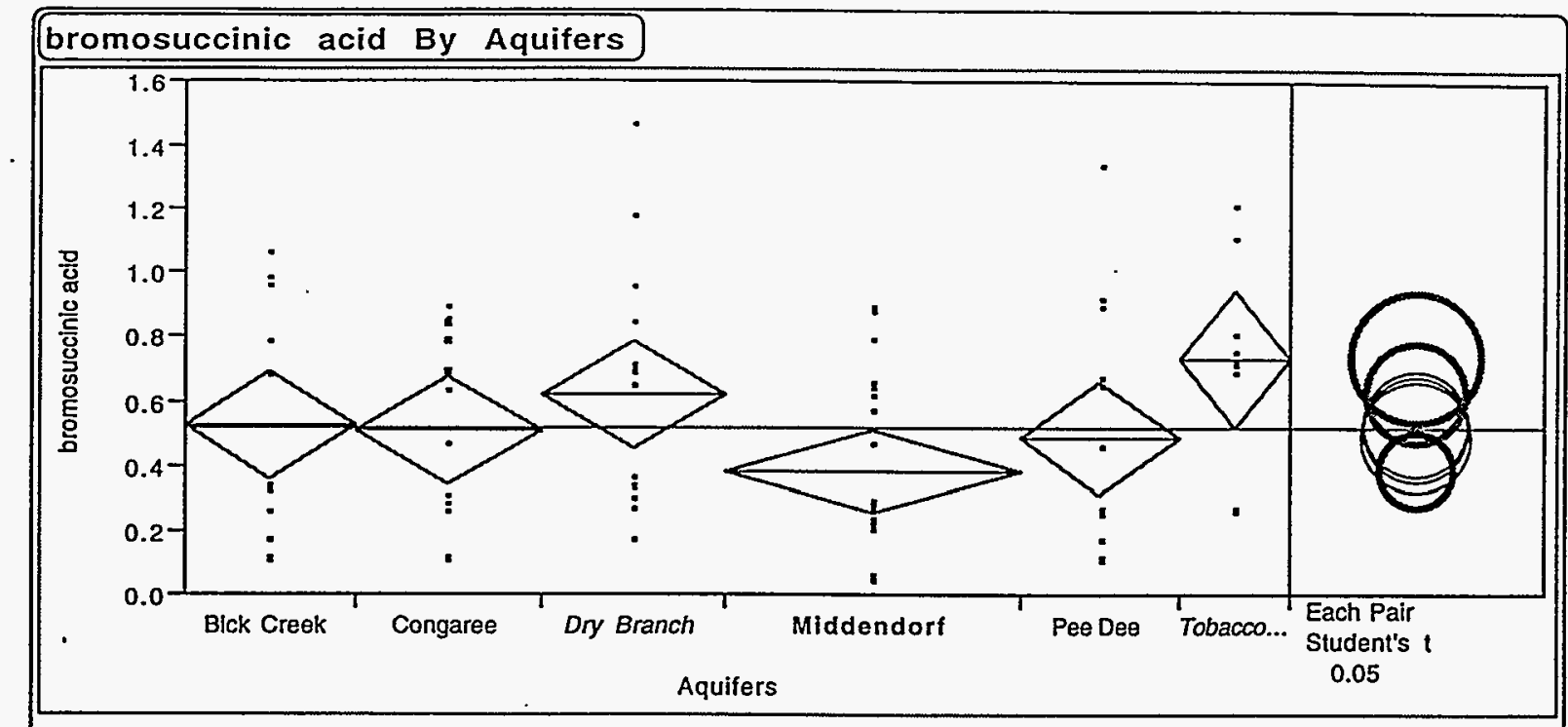

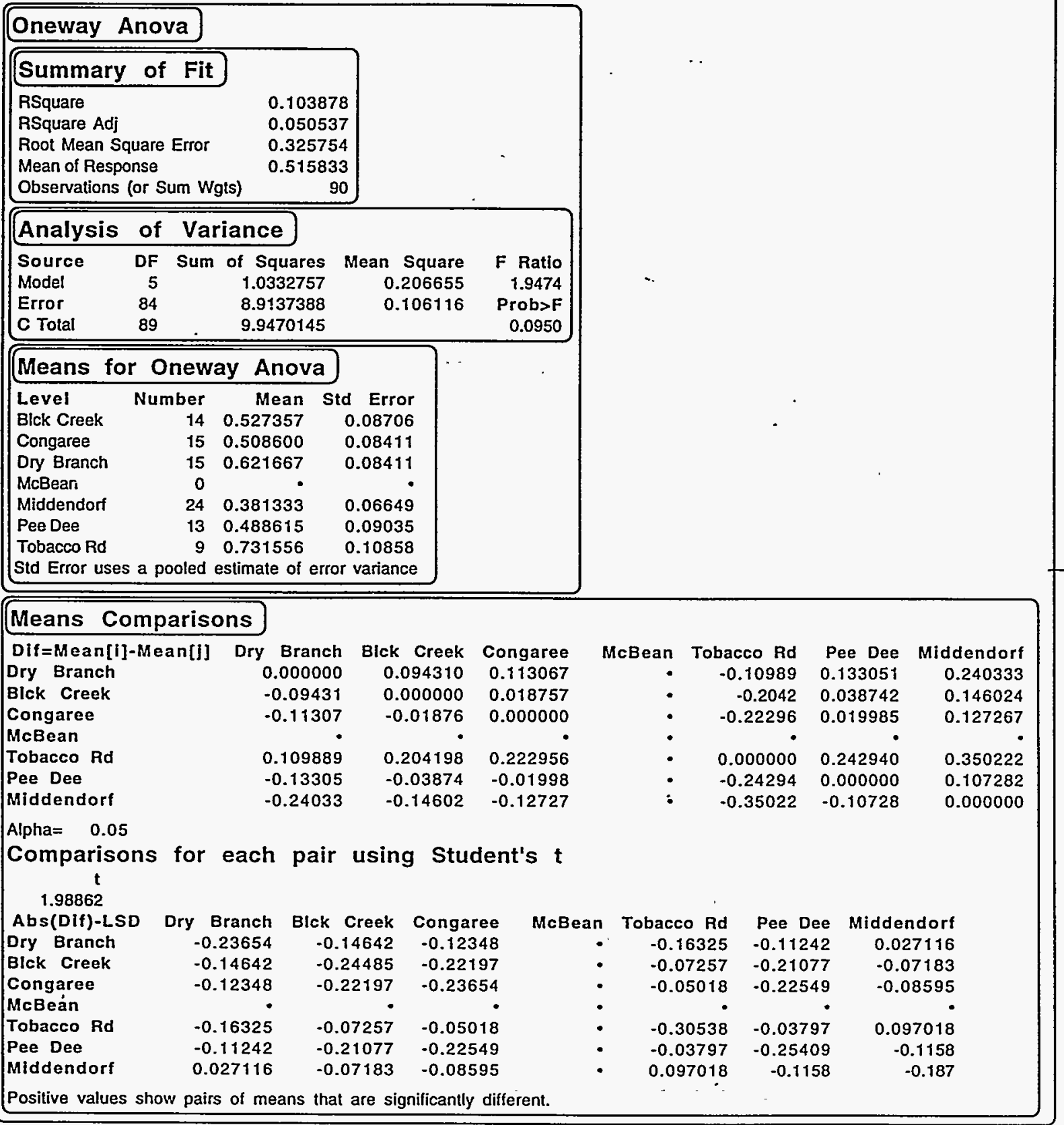


FIGURE 19.

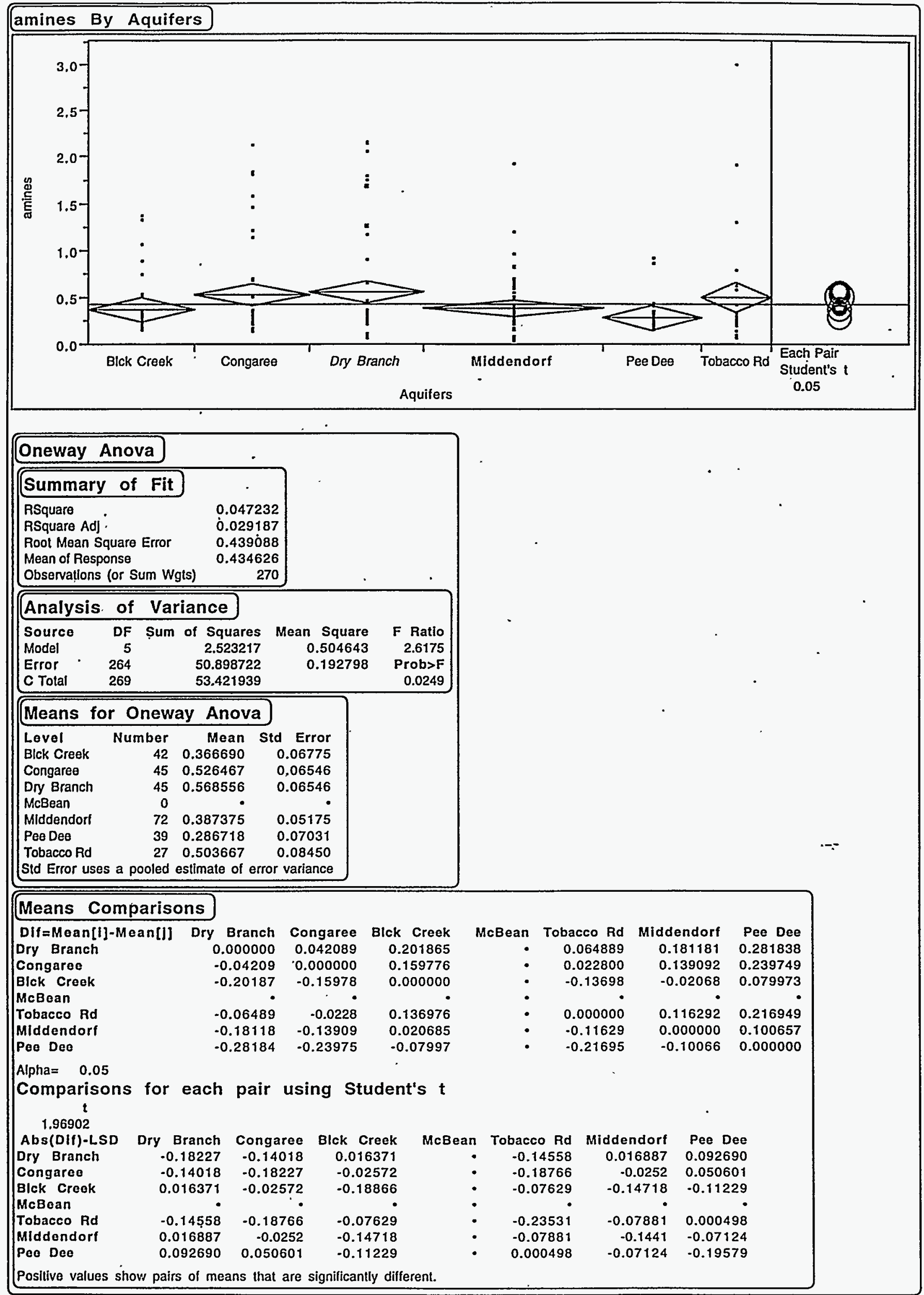


FIGURE $19 \mathrm{a}$.

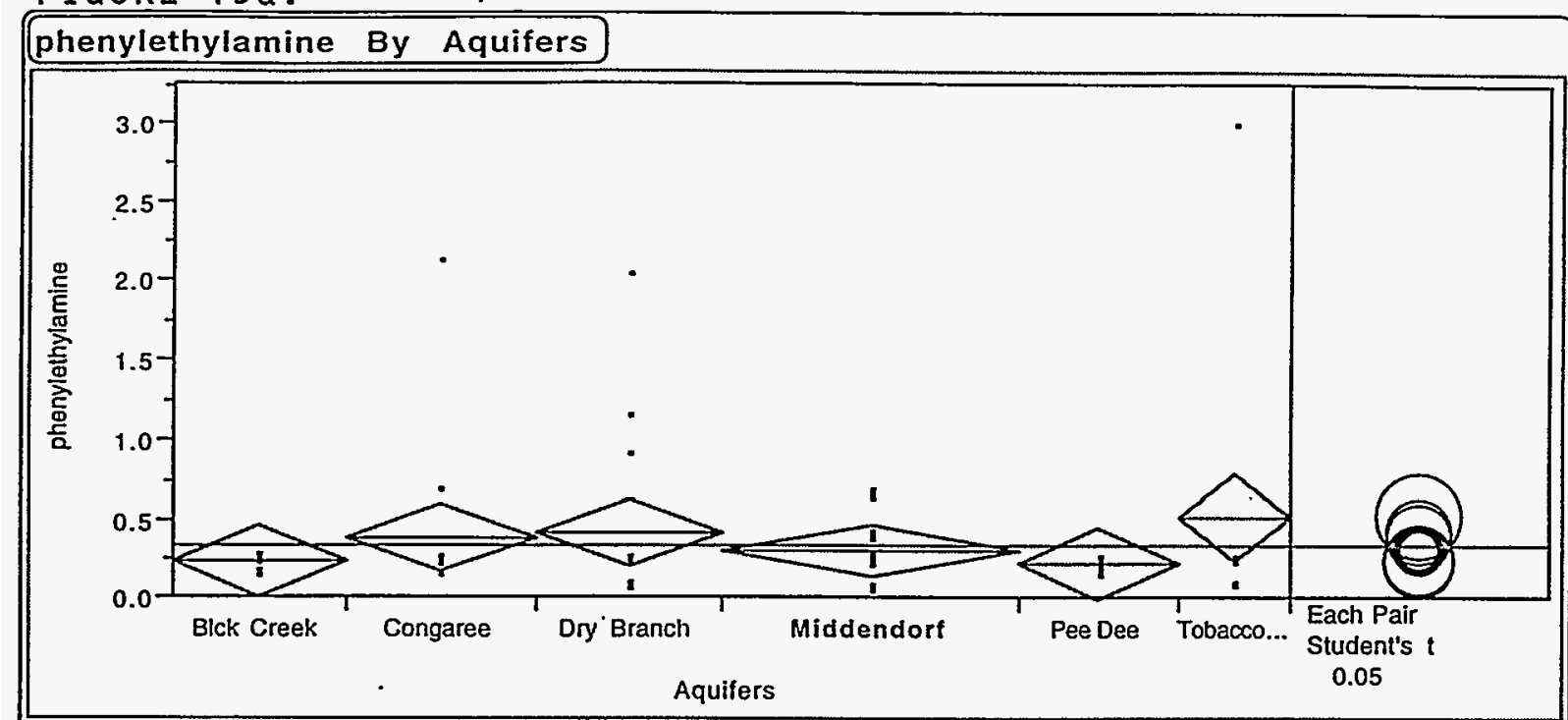

\begin{tabular}{|lr|}
\hline Oneway Anova \\
\hline Summary of Fit \\
\hline RSquare & \\
RSquare Adj & 0.046606 \\
Root Mean Square Error & -0.01014 \\
Mean of Response & 0.428675 \\
Observations (or Sum Wgts) & 0.335267 \\
\hline
\end{tabular}

\begin{tabular}{|lrrrr|}
\hline Analysis & of & Variance & & \\
Source & DF . Sum of Squares & Mean Square & F Ratio \\
Model & 5 & 0.754584 & 0.150917 & 0.8213 \\
Error & 84 & 15.435999 & 0.183762 & Prob $>$ F \\
C Total & 89 & 16.190584 & & 0.5380 \\
\hline
\end{tabular}

Means for Oneway Anova

Level Number Mean Std Erro

$\begin{array}{lrrr}\text { Blck Creek } & 14 & 0.236500 & 0.11457\end{array}$

$\begin{array}{lll}\text { Congaree } & 15 & 0.379933\end{array}$

$\begin{array}{llll}\text { Dry Branch } & 15 & 0.419600 & 0.11068\end{array}$

McBean

Middendorf

Pee Dee

$24 \quad 0.304000$

Tobacco Rd

$9 \quad 0.517333$

0.11889

0.14289

Std Error uses a pooled estimate of error variance

\section{Means Comparisons}

Dif=Mean[i]-Mean[j] Dry Branch

Dry Branch

Congaree

Blck Creek

McBean

Tobacco Rd

Middendorf

Pee Dee

Alpha $=0.05$

0.000000

$-0.0396$

$-0.1831$

Congaree

0.039667

0.000000

$-0.14343$

0.097733

$\begin{array}{ll}-0.1156 & -0.07593\end{array}$

$-0.19514$

$-0.15547$
Blck Creek

0.183100

0.143433

0.000000

0.280833

0.067500

$-0.01204$
McBean Tobacco Rd Middendorf - $\quad-0.09773$

$-0.1374$

$-0.28083$

- 0.000000

$-0.21333$

- $\quad-0.29287$
0.115600

0.075933

$-0.0675$

0.213333

0.000000

$-0.07954$
Pee Dee

0.195138

0.155472

0.012038

0.292872

0.079538

0.000000

Comparisons for each pair using Student's t

1.98862

Abs(Dif)-LSD

Dry Branch

Congaree

Bick Creek

McBean

Tobacco Rd

Middendorf

Pee Dee

Pee Dee
Dry Branch
$-0.31128$
$-0.27161$
$-0.13369$
Congaree
$-0.31128$
$-0.17335$
$-0.2617$
$-0.16498$
$-0.12789$
$-0.22203$
$-0.20465$
$-0.16756$
Blck Creek
$-0.13369$
$-0.17335$
$-0.3222$
$-0.08338$
$-0.21918$
$-0.3163$

McBean Tobacco Rd Middendorf

$-0.20465 \quad-0.16756$

$-0.21918-0.3163$

$-0.11987-0.07678$

$-0.24609-0.21403$

$\begin{array}{ll}-0.21403 & -0.33437\end{array}$ 
FIGURE 19b.

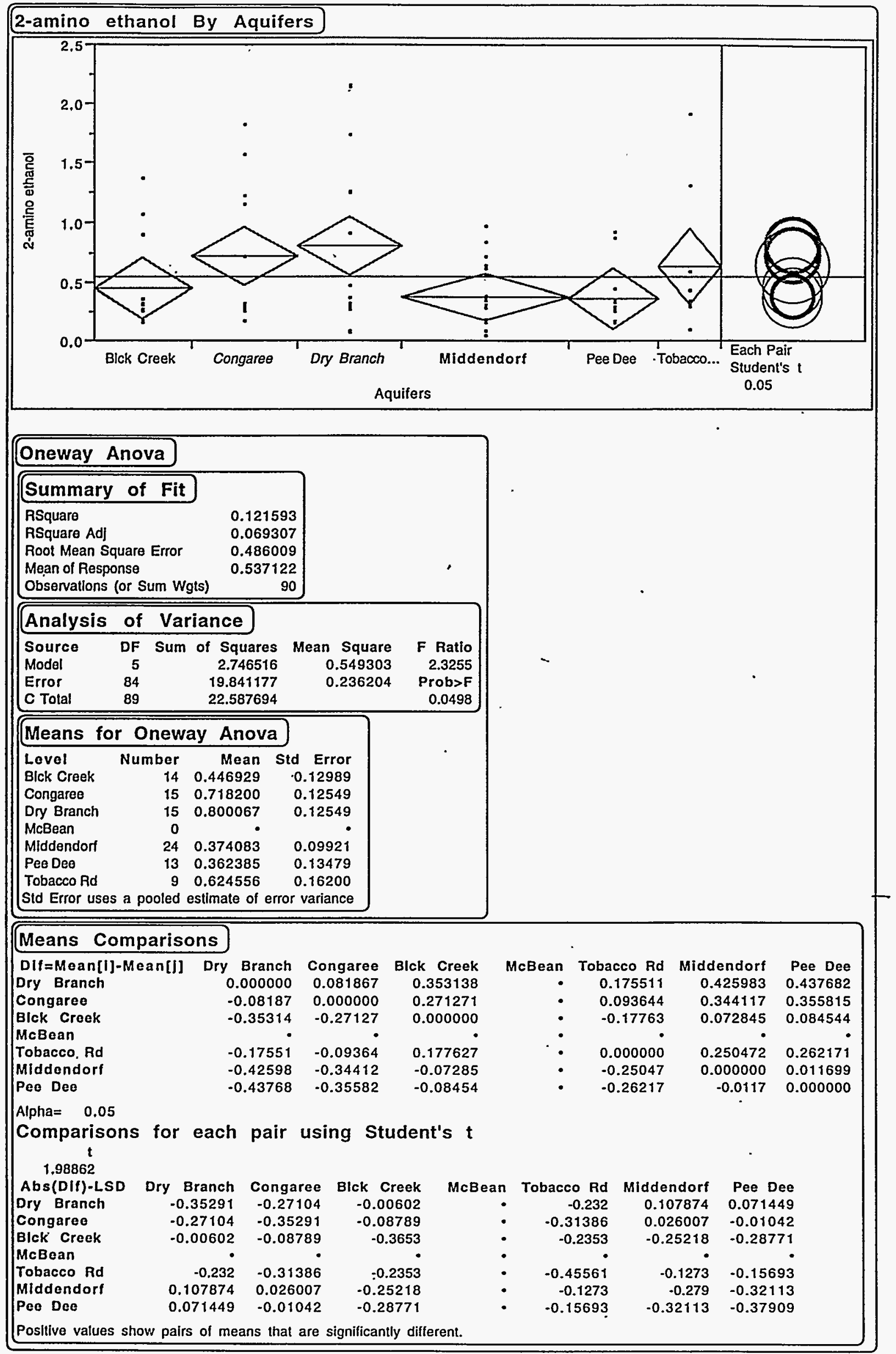


FIGURE 19c.

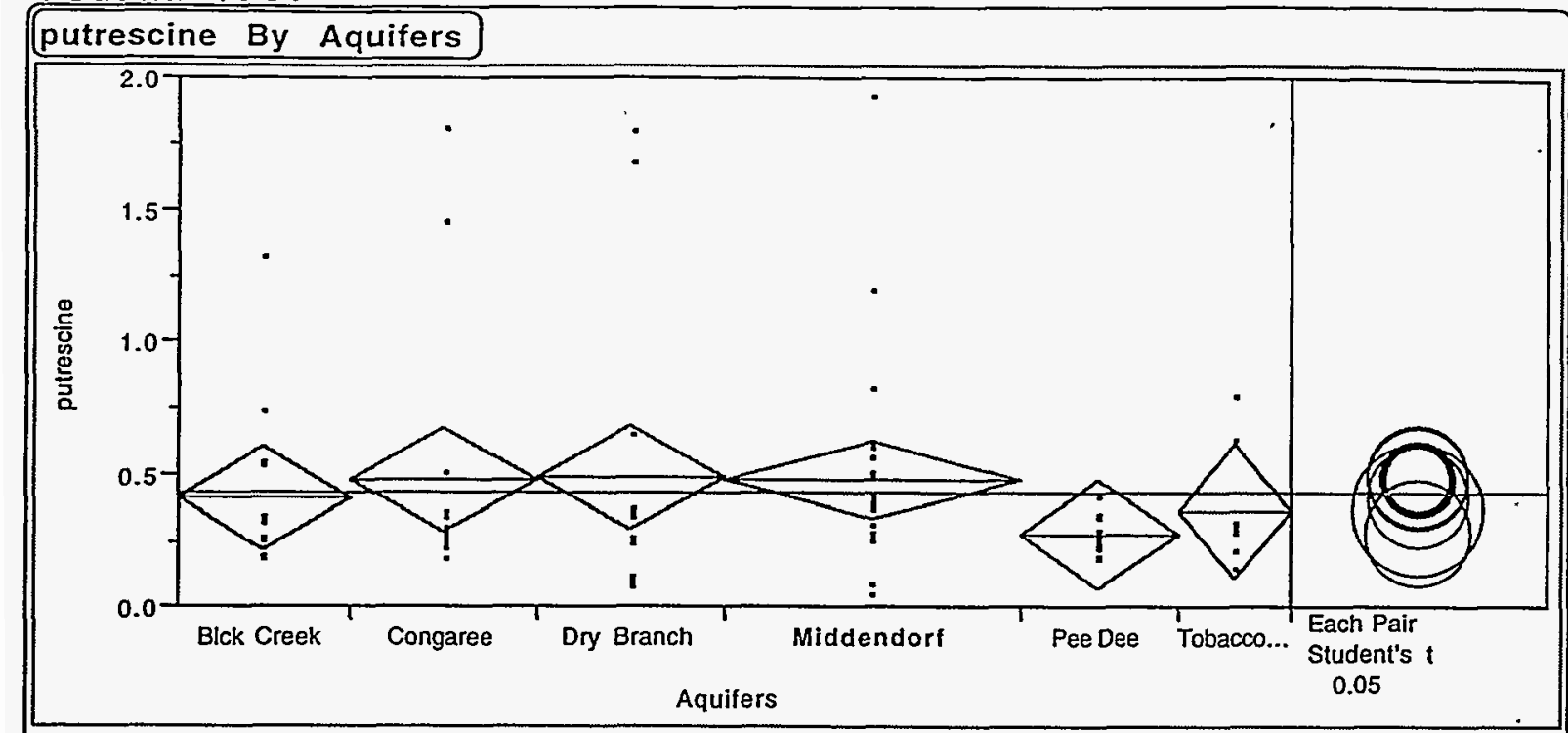

\section{Oneway Anova}

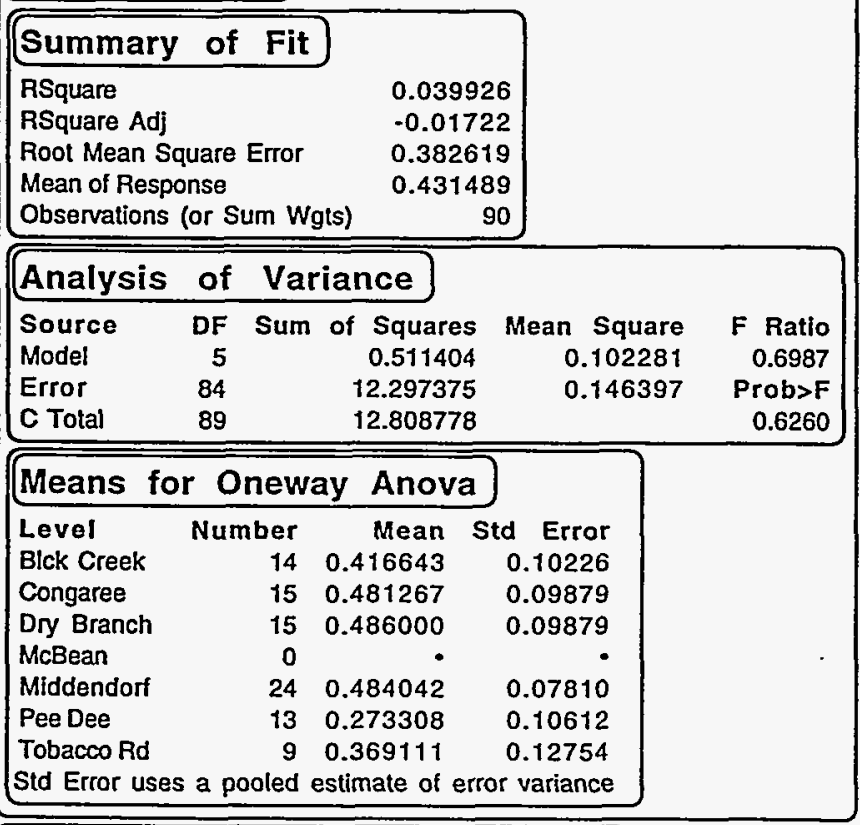

\section{Means Comparisons}

\begin{tabular}{|c|c|c|c|c|c|c|c|}
\hline Dif =Mean[i]-Mean[j] & Dry Branch & Middendorf & Blck Creek & McBean & Congaree & Tobacco Rd & Pee Dee \\
\hline Dry Branch & 0.000000 & 0.001958 & 0.069357 & $\bullet$ & 0.004733 & 0.116889 & 0.212692 \\
\hline Middendorf & -0.00196 & 0.000000 & 0.067399 & $\bullet$ & 0.002775 & 0.114931 & 0.210734 \\
\hline Blck Creek & -0.06936 & -0.0674 & 0.000000 & $\bullet$ & -0.06462 & 0.047532 & 0.143335 \\
\hline McBean & • & - & - & - & - & - & - \\
\hline Congaree & -0.00473 & -0.00278 & 0.0064624 & $\bullet$ & 0.000000 & 0.112156 & 0.207959 \\
\hline Tobacco Rd & -0.11689 & -0.11493 & -0.04753 & - & -0.11216 & 0.000000 & 0.095803 \\
\hline Pee Dee & -0.21269 & -0.21073 & -0.14334 & - & -0.20796 & -0.0958 & 0.000000 \\
\hline
\end{tabular}

Alpha $=0.05$

Comparisons for each pair using Student's $t$ $t$ 1.98862

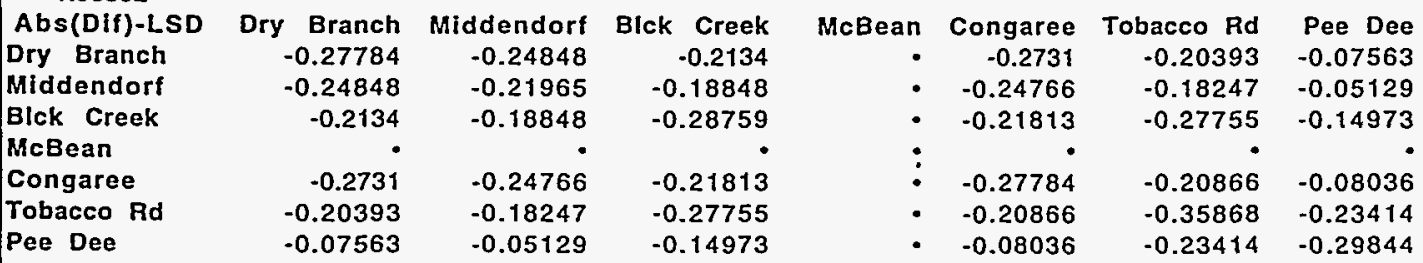

Positive values show pairs of means that are significantly different. 